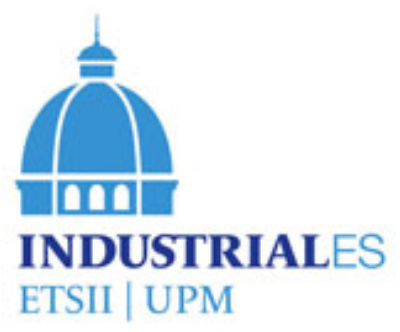

ESCUELA TECNICA SUPERIOR DE INGENIEROS INDUSTRIALES

\title{
DEVELOPMENT OF STRATEGIES TO REDUCE ENERGY EXPENDITURE FOR LOWER-LIMB ACTIVE ORTHOSES
}

\author{
DOCTORAL THESIS \\ Daniel Sanz Merodio \\ Electronic Physicist \\ Director \\ Dr. Elena Garcia Armada
}

PhD Robotics and Automation, Industrial Engineer 



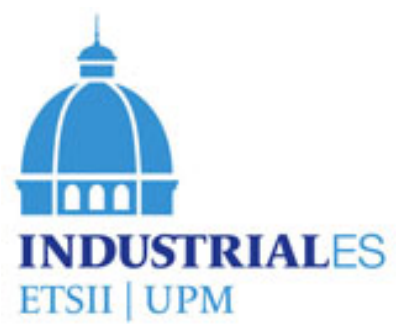

ESCUELA TECNICA SUPERIOR DE INGENIEROS INDUSTRIALES

\title{
DEVELOPMENT OF STRATEGIES TO REDUCE ENERGY EXPENDITURE FOR LOWER-LIMB ACTIVE ORTHOSES
}

\author{
DOCTORAL THESIS \\ Daniel Sanz Merodio \\ Electronic Physicist \\ Director \\ Dr. Elena Garcia Armada \\ PhD Robotics and Automation, Industrial Engineer
}

Madrid, September, 2018 
Development of strategies to reduce energy expenditure for lower-limb active orthoses

Author: Daniel Sanz Merodio

Advisor: Dr. Elena Garcia Armada

First edition, August 2018 
To Celsa, Gemma, Cecilia and Martina 



\begin{abstract}
Lower-limb exoskeletons and powered orthoses are non-invasive devices that assist patients with locomotive disorders to achieve correct limb movements. The intended use as an assistive device for daily life activities still encounters barriers to practical implementation. Current batteries cannot meet the long-term power requirements for these devices, which need to operate for long periods. This issue has become a major challenge in the development of these portable robots. Conversely, legged locomotion in animals and humans is efficient. This thesis explores the methods to reduce energy consumption in the motion control of gait exoskeletons and especially its applicability and feasibility. From a thorough analysis of the human gait from the energetic point of view and based on the state of development of robotics, a hybrid strategy is proposed to reduce energy consumption in the gait cycle.

Three major research areas can be distinguished in energy-efficient biped walking robots:

- The first research area focuses on developing optimal gait trajectories for active walking robots. These trajectories are obtained by means of optimization procedures to minimize an objective function.

- The second research area focuses on exploiting the passive-dynamics of the robot legs. In the first instance, the research is focused on non-active mechanisms that descend ramps taking advantage of the potential energy and the elastic energy of the ground contact. Subsequently, by applying these concepts, it is possible to make robots walk on flat ground providing only the amount of energy
\end{abstract}


previously contributed by gravity, to take advantage of the elastic energy of the ground contact.

- In the third area, leg dynamics is modified by including passive or active elastic mechanisms in the joints to reduce energy losses caused by the impact with the ground and to store and release energy for an improved energy-efficiency.

In this thesis, a hybrid approach is proposed, taking advantage of the benefits of the above listed strategies to achieve an energy efficient gait. An hybrid approach is needed because gait exoskeletons applied to gait rehabilitation or assistance of people with neuromuscular diseases must respect the constraints imposed by the physiological needs of the patient. To solve this requirement the gait control approach must explode the natural dynamics of the leg; actively modifying joints dynamics by the use of Variable Stiffness Actuators (VSA).

By the application of a strategy of stiffness variation, control strategies based on passive dynamics can be implemented in the same joint, while the necessary torque can be provided by a stiff position controller in phases of higher torque demand, allowing subactuation in gait phases where inherent inertia is enough to take advantage of it and also providing the high torque necessary for the orthosis to perform more versatile and non-cyclic movements, such as sitting and standing maneuvers. In turn, by establishing the adequate stiffness to the joint, energy consumption is reduced in changes of direction of the joint and energy can be stored and released in certain gait phases.

The locomotion control strategies proposed have been validated through their implementation in the ATLAS active orthosis prototype. In this experiment, the importance of the variation of stiffness in relation to an efficient gait is reflected. This hybrid strategy achieves a reduction in energy consumption of $40 \%$, while maintaining the robustness and reliability of the active orthosis with stiff actuators. 
The results of this research work have led to 7 publications in indexed scientific journals and 19 articles in international conferences, several of which have been awarded for their scientific and technical excellence. The doctoral thesis has had a relevant impact in the area of rehabilitation robotics since its results are being used in real environments, since pediatric gait exoskeletons, transferred to the technologybased company Marsi Bionics, are being used in two hospitals in the therapy of neuromuscular diseases in childhood. 



\section{Resumen}

El uso de las órtesis activas es cada vez más habitual tanto en el campo de la rehabilitación como asistencial. Las órtesis activas o exoesqueletos de asistencia a la marcha humana son dispositivos de uso externo para pacientes con problemas de movilidad que aportan la fuerza y movilidad que le falta al usuario. El uso de estos dispositivos puede ser óptimo utilizado a diario, integrado en el mayor número de actividades cotidianas posible. Pero las órtesis activas comerciales actuales no tienen autonomía suficiente para proporcionar la potencia consumida durante toda una jornada. Las baterías actuales no proporcionan los requerimientos de potencia que demandan estas órtesis activas. Aumentar la autonomía energética se ha convertido en uno de los grandes retos para el desarrollo de estos estos dispositivos. En general, el consumo energético de los robots bípedos de uso general es significativamente mayor que el de los humanos u otros bípedos mamíferos. A partir de un minucioso análisis de la marcha humana y la anatomía de la locomoción desde el punto de vista energético y basándose en el estado del arte de la robótica, en esta tesis se propone una estrategia híbrida para reducir el consumo energético en la marcha, primando la aplicabilidad y la fiabilidad para implementarla en el exoesqueleto ATLAS.

En el campo de los robots bípedos se distinguen fundamentalmente tres estrategias de eficiencia energética:

1. Optimizar las trayectorias a seguir por el extremo de la pierna para minimizar una función objetivo relacionada con la energía consumida. 
2. Aprovechar la dinámica pasiva (energía potencial y cinética) de la pierna del robot para realizar el movimiento subactuado o con poca inyección de energía.

3. En el diseño de la extremidad robótica, modificar su dinámica incluyendo elementos y mecanismos elásticos en las articulaciones para reducir las pérdidas de energía en los impactos con el suelo y para acumular y soltar energía en las fases de la marcha donde el comportamiento energético de las articulaciones sea el mismo que el de un elemento elástico.

En esta tesis se propone una aproximación híbrida, que trata de aunar los beneficios de cada estrategia anterior.

Habitualmente los pacientes objetivo, hacia los que está enfocado el diseño de estos exoesqueletos, tienen límites articulares importantes, flexos de cadera, rodilla y una marcada limitación en el rango de flexión en el tobillo. Así, la primera estrategia presentada no es directamente aplicable, puesto que la trayectoria del extremo efectivo en las órtesis activas debe calcularse respetando las restricciones anatómicas del paciente. La segunda y tercera estrategias planteadas se logran en esta tesis proponiendo e implementado un esquema de control de rigidez en un actuador de rigidez variable (VSA).

Gracias a esta estrategia de variación de rigidez se pueden implementar en una misma articulación estrategias de control basadas en la dinámica pasiva en la marcha, mientras que se puede aportar el par necesario para un control rígido en posición en fases de mayor demanda de par, permitiendo así subactuación en fases de la marcha donde la inercia inherente al movimiento es suficiente para mantenerlo y también aportando el alto par necesario para que la órtesis realice movimientos más versátiles y no cíclicos, como maniobras de sentar y levantar al paciente. A su vez, estableciendo la rigidez adecuada a la articulación, se reduce el consumo en los cambios de dirección de la articulación y se puede almacenar y liberar energía en ciertas fases. 
Las estrategias de control de la locomoción propuestas se han validado a través de su implementación en el prototipo de órtesis activa ATLAS. En esta experimentación se refleja la importancia de la variación de rigidez en relación con una marcha eficiente. Esta estrategia híbrida logra una reducción en el consumo energético de $40 \%$, mientras se mantiene la robustez y fiabilidad de la órtesis activa con actuadores rígidos.

Los resultados del trabajo de investigación han dado lugar a siete publicaciones en revistas científicas indexadas y 19 artículos en congresos internacionales, varios de los cuales han sido premiados por su excelencia científica y técnica. La tesis doctoral ha tenido un impacto relevante en el área de la robótica de rehabilitación ya que sus resultados están utilizándose en entornos reales, dado que los exoesqueletos de marcha pediátricos, transferidos a la empresa de base tecnológica Marsi Bionics, están siendo utilizados en dos hospitales del país en la terapia de enfermedades neuromusculares en la infancia. 



\section{Acknowledgements}

I would like to begin by giving thanks the Centre for Automation and Robotics UPM-CSIC in which this thesis was carried out.

I also wish to thank my research adviser, Dr. Elena Garcia for her direction, suggestions, patience, advice and the opportunity and privilege of working in this project.

My gratitude also extends to my coworkers (Juan Carlos, Manuel, Xavi, Luis, Leonel, Mariano, etc.) for their dedication, advises, help and good moments that we have labored together in the past years on common work.

Thanks all my fellows at Marsi Bionics for their support and understanding for the completion of this thesis.

I thank my parents and brother for their constant support and encouragement during the development of this work.

Finally, I thank the one to whom I dedicate this dissertation, my wife, Gemma. Her love, support, and patience have encouraged me in each step of this thesis.

Muchas gracias,

Daniel Sanz Merodio

August 2018 



\section{Contents}

List of Figures

List of Tables $\quad$ Xxix

1 Introduction 1

1.1 Introduction . . . . . . . . . . . . . . . 1

1.2 Causes of mobility impairment . . . . . . . . . . 2 2

1.3 Robotic gait exoskeletons . . . . . . . . . . . . . . . . 7

1.4 Thesis objective . . . . . . . . . . . . . . . . 17

1.5 ATLAS active orthosis as a test-bed for improved energy consumption exoskeletons . . . . . . . . . . . . . . . . . 18

1.6 Thesis outline . . . . . . . . . . . . . . . . . 20

2 Human Gait 23

2.1 Introduction . . . . . . . . . . . . . . 23

2.2 Comparison of energy consumption of active orthoses. Cost of transport COT . . . . . . . . . . . . . . . . 26 26

2.3 Definitions and nomenclature . . . . . . . . . . . 28

2.4 The human gait cycle . . . . . . . . . . . . . . . 30

2.5 Strategies and mechanisms to reduce energy expenditure in human walking ....................... 32

2.5.1 Kinematics of human walking . . . . . . . . 32

2.5.1.1 Kinematics of the center of mass of the body . . 33

2.5.1.2 Kinematics of the leg joints . . . . . . . . 33 
2.5.2 Kinetics of human walking . . . . . . . . . . 34

2.6 A model of human gait . . . . . . . . . . . . . 36

2.6.1 Kinematic model of walking . . . . . . . . . . 38

2.6.1.1 Providing ground clearance . . . . . . . . 42

2.6.1.2 Providing an adequate step length . . . . . . . 43

2.6.1.3 Support of the body weight ........ . 44

2.6.1.4 Smooth phase transitions ........ . 46

2.7 Model of the human musculoskeletal system . . . . . . . . . . 49

2.7.1 Muscle model . . . . . . . . . . . . . . 50 50

2.7.2 Model of muscle-tendon unit (MTU) . . . . . . . 50 50

2.8 Conclusions . . . . . . . . . . . . . . . . 53

3 Biped Robot Strategies To Reduce Energy Consumption 55

3.1 Introduction . . . . . . . . . . . . . . . 55

3.2 Economy vs. versatility, a trade-off . . . . . . . . . . 55

3.3 High energy consumption of stiff actuators . . . . . . . . . 58

3.4 Variation of actuator compliance to reduce energy consumption . . 61

3.4.1 Series Elastic Actuator . . . . . . . . . . . . . . . . 61

3.4.2 Antagonistic-Controlled Stiffness . . . . . . . . . 62

3.4.3 Variable Stiffness Actuators (VSA) . . . . . . . . . 65

3.4.4 The preload adjustment of single spring . . . . . . . 65

3.4.5 Changing transmission between load and spring . . . . . . 66

3.5 Conclusions and control challenges . . . . . . . . . . . . 70

4 Analysis of Energy Efficient Gait Approaches For Exoskeletons 73

4.1 Introduction . . . . . . . . . . . . . . . 73

4.2 Methodology to analyse energy consumption . . . . . . . . . 74

4.3 Measuring gait energy expenditure . . . . . . . . . . 77

4.3.1 Static optimization method . . . . . . . . . . 78

4.3.2 Dynamic parameter optimization . . . . . . . . . . 80

4.4 Exploiting passive pendulum dynamics during swing and stance . 85

4.5 Storing and releasing energy in elastic mechanisms . . . . . . . 87

4.5.1 Storing energy at hip and ankle . . . . . . . . . 88

4.5.2 Knee damping ................. . . . . 91 
4.5.3 Knee locking . . . . . . . . . . . . . . 92

4.6 The combined approach . . . . . . . . . . . . 93

4.7 Feasibility of the presented proposals . . . . . . . . 95

5 Synergic Multiarticular Actuation 99

5.1 Introduction . . . . . . . . . . . . . . . 99

5.2 Ankle motion along the gait cycle . . . . . . . . . . 101

5.2.1 Providing toe-off impulse . . . . . . . . . . 102

5.2.2 Providing ground clearance in the swing phase . . . . . 103

5.3 Prototype implementation . . . . . . . . . . . . . . 106

5.4 Experimental evaluation . . . . . . . . . . . . 107

5.4 .1 Leg behavior in phase $1 \ldots \ldots \ldots$

5.4 .2 Leg behavior in phase $2 \ldots \ldots \ldots$

5.4 .3 Leg behavior in phase $3 \ldots \ldots$. . . . . . . . 108

5.5 Conclusions and application remarks . . . . . . . . . . 109

6 Variable Stiffness Actuation 111

6.1 Joint requirements for active-orthoses . . . . . . . . . . 112

6.1.1 Actuator requirements at the hip . . . . . . . . . 112

6.1.2 Actuator requirements at the knee . . . . . . . . . 114

6.1.3 Actuator requirements at the ankle . . . . . . . . 114

6.2 Joint stiffness variation in the gait cycle . . . . . . . . . . 117

6.2.1 Hip stiffness variation . . . . . . . . . . . . 117

6.2.2 Knee stiffness variation . . . . . . . . . . . . . 118

6.2.3 Ankle stiffness variation . . . . . . . . . . . . . 120

6.2.4 General impedance considerations . . . . . . . . . 122

6.3 Adjustable stiffness with embedded sensor (ARES) actuator . . . 124

6.4 Working principle of the variable stiffness actuator . . . . . . . 125

6.5 Joint speed control . . . . . . . . . . . . . . . 127

6.6 Force measurement embedded in the ARES actuator . . . . . . 129

6.7 Simulation-based performance evaluation . . . . . . . . . 130

6.8 ARES prototype . . . . . . . . . . . . . . . . 131

6.9 Conclusions . . . . . . . . . . . . . . . . . 134 
7 Energy-efficient control of locomotion 135

7.1 Introduction . . . . . . . . . . . . . . 135

7.2 Hibrid control approach . . . . . . . . . . . . 135

7.3 Joint-level control system . . . . . . . . . . . . . . . . 138

7.3.1 Stiffness control at the hip . . . . . . . . . . 139

7.3.2 Stiffness control at the knee . . . . . . . . . . 141

7.3.3 Stiffness control at the ankle . . . . . . . . . . . 144

7.4 Experimental assessment of the control approach . . . . . . . 147

7.4.1 Experimental setup . . . . . . . . . . . . 147

7.4.2 Force measurement validation . . . . . . . . . . 148

7.4.3 Hip stiffness control . . . . . . . . . . . . . . 151

7.4.3.1 Experimental validation of ARES at the hip . . 151

7.4.4 Knee stiffness control . . . . . . . . . . . . 153

7.4.4.1 Complete knee gait cycle . . . . . . . . 157

7.4.5 Ankle stiffness control . . . . . . . . . . . . . . 161

7.4.6 Variable stiffness control in the ATLAS active orthosis . . 165

7.5 Conclusions . . . . . . . . . . . . . . 167

8 Conclusions 169

8.1 Main contributions . . . . . . . . . . . . . 171

8.1 .1 Publications . . . . . . . . . . . . 172

8.1.1.1 Journal publications . . . . . . . . . . 172

8.1.1.2 International Conference publications . . . . . 173

8.2 Technology transfer of thesis results . . . . . . . . . . 175

8.2.1 Patents ................... 175

A ATLAS active orthosis 179

A.1 Kinematic analysis . . . . . . . . . . . . . . . . . . . . . . . . . . 181

A.1.1 Direct kinematics . . . . . . . . . . . . . . . . 181

A.1.2 Inverse kinematics . . . . . . . . . . . . 182

A.2 Dynamic model of the active orthosis ATLAS . . . . . . . . . . 184

A.3 Mechanical configuration . . . . . . . . . . . . . 186

A.4 Electrical architecture and sensorial system . . . . . . . . . . . 188

A.5 Gait trajectory generation . . . . . . . . . . . . . . 189 
A.6 Control scheme . . . . . . . . . . . . . . . . . . . 191

B Walker concept and design 195

Bibliography 199 



\section{List of Figures}

1.1 Most common causes of paralysis.[1] . . . . . . . . . . 6 6

1.2 Force amplification exoskeleton, HULC, XOS-2. . . . . . . . 9

1.3 Force amplification exoskeleton, NTU-LEE and HAL-5. . . . . . 9

1.4 Representative BWS robotics rehabilitation: Lokomat by Hocoma (left) and LOPES by the University of Twente (right). . . . . . . . 12

1.5 Rehabilitation robot ALEX by University of Delaware (left) and Walktrainer by Swiss Foundation (right). . . . . . . . . . 13

1.6 Active othoses, Rewalk, REX and Ekso. . . . . . . . . . . . . . . 14

1.7 Under-actuated exoskeleton from MIT. . . . . . . . . . . . 17

1.8 The ATLAS active orthosis prototype . . . . . . . . . 19

2.1 Rate of energy consumption versus speed for human walking and running on level (0\%), uphill ( $+5 \%$ gradient) and down hill $(-5 \%$ gradient).[2] . . . . . . . . . . . . . . . . . . . . . . . 24

2.2 Energy consumption per unit of distance in walking at different gradients. For each gradient, a minimum occurs at a given speed.[2] 24

2.3 Oxygen consumption of a horse running at a range of speeds in three gaits.[3] . . . . . . . . . . . . . . 25

2.4 Comparison of the cost of transport for humans, animals, vehicles and robots. Also the graph includes the cost of transport of some of the active orthosis. . . . . . . . . . . . 27 27

2.5 Anatomical planes of the body. . . . . . . . . . . . 29

2.6 Nomenclature of sagittal joint motions. . . . . . . . . . . . . . 29

2.7 Complete gait cycle with detailed phases. St: Stance. Sw: Swing . 30 


\section{LIST OF FIGURES}

2.8 The movement of the COM of the body when walking on level ground in the sagittal plane follows a sinusoid.[4] . . . . . . 33

2.9 Angle, moment exerted and mechanical power of each joint in a gait cycle in the sagital plane $[5] \ldots \ldots \ldots$. . . . . . . . 35

2.10 Model of inverted pendulum for single support. . . . . . . . . . 40

2.11 Joint angle patterns assuming the body pivoting on the ankle joint. The thin line represents the movement of each joint to follow the pattern of Clinical Gait Analysis (CGA). The thick line represents the movement of the joints, only in the support phase, assuming the body pivoting on the ankle joint following an inverted pendulum pattern and compensating the movement with the hip to maintain the trunk upright. . . . . . . . . . . . . . . . .

2.12 Horizontal and vertical components of the velocity of the COM at different walking speeds during the stand phase. Black line follows the simple model of inverted pendulum during stance. Grey line represents the normal deviation of the movement of COM in human walking. [6]. . . . . . . . . . . . . . . . . . . . .

2.13 Joint angle patterns to provide ground clearance. The thin line represents the movement of each joint to follow the pattern of Clinical Gait Analysis (CGA). The thick line represents the movement of the joints assuming the body is pivoting on the ankle joint in the support phase, and providing ground clerance in the swing phase.

2.14 Joint angle patterns to provide an adequate step length. The thin line represents the movement of each joint to follow the pattern of Clinical Gait Analysis (CGA). The thick line represents the movement of the joints assuming the body pivoting on the ankle joint, in the support phase, and providing ground clerance in the swing phase. To provide an adequate step length the hip has to have simetrical flexion-extension $(\mathbf{L})$ between the opposite foot and the contact foot. In addition, the knee must have enought flexion and extension at the begining of the double support ( $\mathbf{D}$ and $\mathbf{E}$ ) to provide a large step lenght. . . . . . . . . . . . . . . . . . 


\section{LIST OF FIGURES}

2.15 Ground reaction force of an inverted pendulum model. Due to the centrifugal force, the ground reaction force is less than the body weight. . . . . . . . . . . . . . .

2.16 Ground reaction force of each leg. The average of the GRF of each leg is not enough to reach the body weight. . . . . . . . . 45

2.17 Ground reaction force of both legs together. The average GFR is enough to reach the body weight with a double support phase. . .

2.18 Horizontal heel velocity. At heel strike, it is reduced to zero to achieve a smooth ground contact. . . . . . . . . . . . . . 47

2.19 Joints angle pattern to provide an adequate foot contact. The thin line represents the movement of each joint to follow the pattern of Clinical Gait Analysis (CGA). The thick line represents the movement of the joints assuming the body pivoting on the ankle joint, in the support phase, and providing ground clerance in the swing phase. To provide a smooth swing to stance transition, knee $(\mathbf{F})$ and ankle (G) modify their pattern at the terminal swing and at initial contact.

2.20 The COM velocity redirection is made by the flexion of the ankle of the trailing leg.

2.21 Joint angle patterns to provide plantar flexion at toe-off. The thin line represents the movement of each joint to follow the pattern of Clinical Gait Analysis (CGA). The thick line represents the movement of the joints assuming the body pivoting on the ankle joint, in the support phase, and providing ground clerance in the swing phase. The impulse of the ankle in plantar flexion $(\mathbf{H})$ provides a smooth transition at the leading heel strike, while the knee flexion (I) improves the kinematics for the impulse. . . . . . . . . . . 49

2.22 Simple model of human muscle (Hill 1993)[7]. . . . . . . . . . . 51

2.23 Simplified model of muscle-tendon unit[7]. . . . . . . . . . 52 52

2.24 Flexion and extension in the elbow. . . . . . . . . . . . 52

3.1 Passived biped robots. (a) Delph University and (b) Cornell Uni-

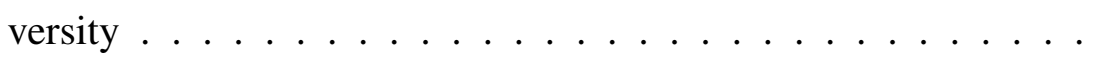




\section{LIST OF FIGURES}

3.2 Model diagram of a DC motor. . . . . . . . . . . . . . 59

3.3 Series Elastic Actuator. Note the spring placed between the motor and the load. . . . . . . . . . . . . . . . . . 61 61

3.4 Control scheme of SEA. With compression of the spring, the force is measured and fedback. . . . . . . . . . . . . . . . . 62

3.5 Migliore's joint with antagonistic setup from the Georgia Institute of Technology. . . . . . . . . . . . . . 63

3.6 Actuator with Mechanically Adjustable Series Compliance (AMASC) from the Carnegie Mellon University. . . . . . . . . . . . . 64

3.7 Quasi-Antagonistic Joint (QA-Joint) from the German Institute of Robotics and Mechatronics (DLR). . . . . . . . . . . . . . 64

3.8 a) VSA Prototype b) Tensioning Mechanism. . . . . . . . . . 6 65

3.9 a) MACCEPA Basic Configuration. b) MACCEPA Prototype. c) MACCEPA 2.0 Schematic Drawing . . . . . . . . . . . . 66

3.10 a) Mechanism of the VS-Joint. b) Working Principle of the VS-Joint. 66

3.11 a) AwAS I Mechanical Principle and b) AwAS II Mechanical Principle. . . . . . . . . . . . . . . . 67

3.12 CompAct-VSA Mechanism. . . . . . . . . . . . . 68

4.1 User with active orthosis simulated with the Yobotics Simulation Construction Set. . . . . . . . . . . . . . . . . . . 76

4.2 Concepts used in genetic algorithms. . . . . . . . . . . . . 82

4.3 Procedure followed in the genetic algorithm applied. . . . . . . . 83

4.4 Gap in the maximum kinetic energy in thigh and shank. . . . . . . 86

4.5 State machine controller. In each state, the PD constants and the transition condition are shown (a) for hip and knee and (b) for the

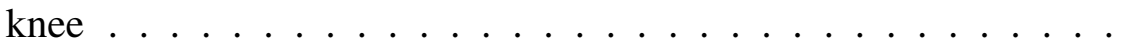

4.6 Torque vs angle in: (a) Hip and (b) Ankle. The torque exerted with a pure CGA position control approach is shown in red. The torque exerted only by the spring is shown in black and the torque exerted when a spring is included is shown in green. . . . . . . . . . . . 


\section{LIST OF FIGURES}

4.7 (A) The power in Conventional Prosthesis is released too early in the push-off phase. When a mechanisms is inlucled (B) to hold the energy and released in the right moment the redirection of the center of mass is more efficient. From $[8]$. . . . . . . . . . . 91

4.8 Finite state machine with passive elements: (a) At the hip ; (b) At the knee . . . . . . . . . . . . . . . . . . . . . 94

5.1 Gait cycledivided into three phases: 1-. Stance, 2-. Toe-off and 3-. Swing . . . . . . . . . . . . . . . . . 101

5.2 Ankle angle depends on the motion in hip and knee. . . . . . . . 102

5.3 Tendons attached to move the ankle (a) A tendon provides the impulse in the ankle. (b) Another tendon provides ground clearance in the swing. . . . . . . . . . . . . . . . . . . . . . . 104

5.4 Exotendons in ATLAS active orthosis prototype: (a) Red tendon providing toe-off impulse; (b) Blue tendon providing ground clearance. Tendon paths have been drawn by lines in the pictures to clarify the illustration. . . . . . . . . . . . . . 106

5.5 Ankle movement based on exotendons in a gait cycle: (a) Right leg support phase and toe-off; (b) Right leg swing phase. . . . . . . . 107

5.6 Angles at the exoskeleton's right leg joints in real experiment. . . 110

6.1 Angle, angular velocity and torque at the hip of children aged 6 to 17 years old walking on flat terrain . . . . . . . . . . . . 113

6.2 Angle, angular velocity and torque at the knee walking on flat terrain 115

6.3 Angle, angular velocity and torque at the ankle walking on flat terrain 116

6.4 Torque vs. position of hip joint walking at slow, normal and fast speeds obtained from [9]. Walking speed normalized to subject's height $\left(\frac{v}{h}\right)$ : slow (blue) for $0.6 \geq \frac{v}{h}<0.8$;medium(red) for $0.8 \geq$ $\frac{v}{h}<1$; fast (green)for $\frac{v}{h} \geq 1$. The support phase is shown in the thickest line. HS - heel strike. LR - Loading response. MSt - Mid Stance. PS - Pre Swing. MSw - Mid Swing . . . . . . . . . . 119 


\section{LIST OF FIGURES}

6.5 Torque vs. position of knee joint walking at slow, normal and fast speeds obtained from [9]. Walking speed normalized to subject's height $\left(\frac{v}{h}\right)$ : slow (blue) for $0.6 \geq \frac{v}{h}<0.8$; medium(red) for $0.8 \geq$ $\frac{v}{h}<1$; fast (green)for $\frac{v}{h} \geq 1$. The support phase is shown in the thickest line. HS - heel strike. LR - Loading response. MSt - Mid Stance. ISw - Initial swing. MSw - Mid Swing . . . . . . . . 121

6.6 Torque vs. position of ankle joint walking at slow, normal and fast speeds obtained from [9]. Walking speed normalized to subject's height $\left(\frac{v}{h}\right)$ : slow (blue) for $0.6 \geq \frac{v}{h}<0.8$;medium(red) for $0.8 \geq$ $\frac{v}{h}<1$; fast (green)for $\frac{v}{h} \geq 1$. The support phase is shown in the thickest line. HS - heel strike. LR - Loading response. MSt - Mid Stance. TSt - Terminal Stance. MSw - Mid Swing . . . . . . . . . 123

6.7 ARES Joint Mechanics. a) CAD Drawing of ARES, b) CAD of Compliant Mechanism, and c) CAD of stiff complex. . . . . . . 125

6.8 ARES Working Principle. a) ARES High Stiffness b) ARES Minimum Stiffness. . . . . . . . . . . . . . . . . . . 126

6.9 Diagram for the calculation of velocities and torques. . . . . . . 128

6.10 ARES Range of Stiffness. a) Torque Range for Different Positions of Lo b) Stiffness Range for Different Positions of Lo.. . . . . . . 132

6.11 ARES Prototype.a) ARES Prototype-Front View. Maximum stiffness. b) ARES Prototype-Front View. Stiffness Near Zero. c) ARES Prototype - Coupling Elements Highlighed . . . . . . . . 133

7.1 Hybrid control scheme ............... 136

7.2 Position control scheme . . . . . . . . . . . . . . 137

7.3 Subactuated control scheme . . . . . . . . . . 137

7.4 Impedance control scheme . . . . . . . . . . . . 138 


\section{LIST OF FIGURES}

7.5 ARES joint-level control diagram. This diagram describes the control scheme of the two motors, the movement of the motor M1 that moves the joint and motor M2 of the stiffness variation. $\theta_{M 1 \text { real }}$ and $\dot{\theta}_{M 1 \text { real }}$ are the actual position and speed of the motor M1 respectively, $\theta_{\text {des }}$ and $\dot{\theta}_{\text {des }}$ are the desired position and speed respectively, and $\theta_{M 2}$ is the angle modification of the angle M1 produced by the motion of M2. $d_{M 2 r e a l}$ and $d_{d M 2}$ are the actual and the desired position of the motor M2, respectively. The desired position is obtained from the desired stiffness Kdes. The velocity sent to the PID controller to the motor M1 is $v_{\text {sent }}$. The position sent to the PID controller to the motor M2 is $p_{\text {sent }} \ldots \ldots$. . . . . . . 140

7.6 State machine controller at the hip. In each state a range of stiffness and the transition condition are shown. . . . . . . . . . . . 141

7.7 Pattern of stiffness implemented in the hip to exploit the intrinsic dynamics of the joint. . . . . . . . . . . . . . . . . 142

7.8 State machine controller at the knee. In each state a range of stiffness and the transition condition are shown. . . . . . . . . . 144

7.9 Pattern of stiffness implemented in the knee to exploit the intrinsic dynamics of the joint. . . . . . . . . . . . . . . 145

7.10 State machine controller at the ankle. In each state a range of stiffness and the transition condition are shown. . . . . . . . . 145

7.11 Pattern of stiffness implemented in the ankle to hold and release the energy. . . . . . . . . . . . . . . . . . . . . 146

7.12 Test-bench to analise hybrid strategies. . . . . . . . . . . . 147

7.13 Experimental control scheme. . . . . . . . . . . . . . . 148

7.14 Torque Tracking in ARES at 2 Levels of Stiffness, (a) low stiffnes (2 Nm/deg) and (b) high stiffness $(10 \mathrm{Nm} / \mathrm{deg})$, with a $1.6 \mathrm{Kg}$ Load. 149

7.15 Torque Tracking in ARES at 2 Levels of Stiffness, (a) low stiffnes (2 Nm/deg) and (b) high stiffness $(10 \mathrm{Nm} / \mathrm{deg})$, with a $3.6 \mathrm{Kg}$ Load. 150

7.16 Position trajectory followed by the hip joint with proposed control varying stiffness to reduce energy consumption. . . . . . . . . 152

7.17 Current consumed at motor M1 varying stiffness to reduce energy following the trajectory shown in Figure 7.16 . . . . . . . . 153 


\section{LIST OF FIGURES}

7.18 CGA pattern utilized as a position reference to the rigid actuator and the pattern commanded to the variable stiffness actuator. . . . 154

7.19 Implementation of the adjustable compliant actuator in the knee joint.155

7.20 Knee stance control(a)Commanded position and the real position reached with the compliant actuator. (b) Current consumption with the rigid actuator and with the variable stiffness actuator. . . . . . 156

7.21 Sequence of the snapshoots with the impemented strategies varying stiffness. . . . . . . . . . . . . . . . 158

7.22 Position followed with proposed control varying stiffness to reduce energy consumption during three gait cycles.

7.23 Current consumed at motor M1 without variation of stiffness (blue) and varying stiffness to reduce energy consumption (red). . . . . . 160

7.24 Current consumed in the motor M2 that change stiffness at the knee

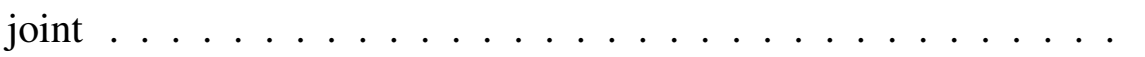

7.25 Test bench for the impemented strategies varying stiffness in the ankle. . . . . . . . . . . . . . . . 162

7.26 Position followed with a control varying stiffness strategy to reduce energy consumption in blue, with fix stiffness $10 \mathrm{Nm} / \mathrm{deg}$ in green and position reference in red. . . . . . . . . . . . . . .

7.27 Current consumed without variation of stiffness (green) and varying stiffness to reduce energy consumption (blue). . . . . . . . . 164

7.28 Current consumed in the motor M2 that changes stiffness at the ankle joint . . . . . . . . . . . . . . 165

7.29 Compliant Actuation in ATLAS Exoskeletons . . . . . . . . . . . 166

8.1 ATLAS 2020 prototype (a) and the ATLAS 2030 commercial(b) exoskeletons . . . . . . . . . . . . . . . . . 177

A.1 Concept of the ATLAS exoskeleton showing main components . . 180

A.2 Front and back views of ATLAS prototype. . . . . . . . . . . 181

A.3 Scheme with the main angles of the leg. . . . . . . . . . . . . . 182

A.4 Scheme for the calculation of inverse kinematics. . . . . . . . . . 183

A.5 Three-link planar manipulator . . . . . . . . . . . . . 184 
A.6 Original foot path obtained by CGA (marker + ) and modified (marker $o$ ) to (a) provide increased ground clearance, (b) reduce body height and (c) reduce step length. . . . . . . . . . . . . . 190

A.7 Smoothing position and velocity curves in the modification of gait.

(a) Angles and speeds at the knee for the original CGA path. (b) Angles and speeds at the knee when the gait is modified to provide larger ground clearance without using Gaussian windows and (c) using Gaussian windows. . . . . . . . . . . . . . . . 192

A.8 General control scheme of the active orthosis. . . . . . . . . . . 194

B.1 The walker adapts to the user's movements while sitting. . . . . 196

B.2 Snapshots of a user in a test while sitting down. . . . . . . . . . 196 



\section{List of Tables}

1.1 Active degrees of freedom of rehabilitation BWS exosketelons. . . 12

1.2 Main features of commercial active orthoses and ATLAS orthosis. In the field of degrees of freedom it is used $\mathrm{A}$ to Active and $\mathrm{P}$ to Pasive ..................... 16

2.1 Cost of transport of several active orthoses . . . . . . . . . 27

2.2 Energy consumption of the leg joints in each anatomical plane (shown in percentages). Computed from the data of [9]. . . . . . 28

3.1 Actuators Comparison $\ldots \ldots \ldots$. . . . . . . . . 69

4.1 Physical parameter of the simulated a 5 year old patient/user wearing a 8 kilograms exoskeleton. . . . . . . . . . . 75

4.2 Glossary of the concepts used in the optimization by genetic algorithms. . . . . . . . . . . . . 81

4.3 Results of applying a passive control strategy. . . . . . . . . 86

4.4 Values for spring constants $k_{\text {spring }}$ and threshold angle $\theta_{\text {thres }}$ in hip and ankle joints. . . . . . . . . . . . . . 89

4.5 Results of including passive elements to a passive control strategy. 92

4.6 Comparison of specific resistance in several bipeds. . . . . . . . . 95

6.1 Motor-gearbox set features . . . . . . . . . . . . . . . 124

6.2 Variation of stiffness with actuator arm length . . . . . . . . . 131

6.3 ARES Prototype Mechanical Properties . . . . . . . . . . . . . 134

7.1 Cost of transport of several active orthoses . . . . . . . . . 167 


\section{LIST OF TABLES}

A.1 ATLAS joint limits compared with average human joint range of motion (angles in degrees). . . . . . . . . . . . . . 187 
If I have seen further than others, it is by standing upon the shoulders of giants.

Sir Isaac Newton

\subsection{Introduction}

The main feature of the human gait is an upright posture. From an evolutionary point of view, this gait offers certain advantages as having the head at a higher position in order to improve the vision of predators and prey, thermoregulation, the possibility of having the hands free to manipulate objects while walking, etc. The ancestral nomad human is characterized by walking long distances to find food, being the walking speed not so important. Our ancestors traveled across Africa walking upright five to seven million years ago [10]. So, after several million years of bipedal walking, the human gait has been adapted and optimized both structurally and morphologically.

It is observed that compared to most quadruped mammals, humans gait is more energy inefficient when running at high speed. In as much as human locomotion evolved, energy expenditure was reduced and the gait was adapted for walking long distances. It is noted that human walking is more efficient than the quadrupedal for the same speed and mass [11].

Therefore, when designing gait exoskeletons for gait assistance, the strategies of human gait shall be imitated in order to achieve its features in efficiency and versatility. 
This chapter presents the benefits of active lower limb orthoses for gait assistance of people who have lost mobility and a classification of active orthoses. A common feature in current developments is their high energy consumption while walking compared ot the human gait energy consumption. Aligned with the need of reducing the energy consumption of active orthoses, the objectives and purposes of this doctoral thesis will be defined.

\subsection{Causes of mobility impairment}

The vast amount of years of evolutionary adaptation to bipedal walking has made human gait efficient, agile and versatile. This involves that the loss of walking hability with the consequent impossibility of upright standing, carries serious health problems.

It is estimated that currently $1 \%$ of the world population is affected by some mobility impairment, being wheelchair bounded to move around. So, around 60 million people in the world are limited in autonomy, independence and health [12]. Of these 60 million wheelchair users, approximately $60 \%$ are elderly and the other $40 \%$ have lost mobility due to other diseases[1]. Figure 1.1, shows the distribution of cases of mobility impairment in Europe.

With age the elderly loses progressively muscle strength, coordination, balance and mobility. At the end, the elderly loses autonomy and requires assistance for daily activities. This lack of motion causes several physiological and psychological diseases to the elderly.

Beside aging, the most common causes of paralysis are:

- Neuromuscular diseases. (NMD) This term encompasses about 150 diseases and ailments that impair muscle function, either directly by muscle pathology or by nerve pathology[13]. Some samples of central disorders include, among others:

Spinal Muscular Atrophy (SMA) is the second most common NMD in infancy and the leading cause of infant mortality. Belonging to the rare diseases group, SMA currently has an incidence of $1 /$ 6,000 live births, and has a clear trend towards increased prevalence. It is a purely motor disease. 


\subsection{Causes of mobility impairment}

Symptoms begin in childhood and there are different known clinical forms with great variability in vital and functional prognosis.

In order to account for the differences in age of onset, symptoms, rate of progression and the ability to walk, the SMA is classified into three types[14]:

- Type I, called Wernig-Hoffmann disease, is the most severe form. Children with type I SMA can never sit down and suffer premature death.

- Type II, onset occurs after 6 months of age; The sitting position is possible without help, but not the ability to walk. The severity of type II varies considerably, ranging from patients who are able to just sit down to those who can stand up with orthotics.

- Type III, or Kugelberg Welander disease, occurs after 18 months of age and children acquire the ability to walk, but may lose it at a variable age.

Different complications usually arise during the course of the disease, including joint deformities, scoliosis, respiratory disorders, hip dislocation, osteoporosis, and fractures. The life expectancy of these children is relatively short, mainly due to the deterioration of respiratory function, which is accelerated by the onset of scoliosis, which occurs in $100 \%$ of patients. Therefore, it is very important to prevent musculoskeletal complications, such as joint deformities, muscle spasms, osteoporosis and scoliosis, in order to maintain a good performance while awaiting the results of the investigation Pharmacological treatment. Therefore, it requires a multidisciplinary therapy.

The fact that SMA type III, in which there is still walking capacity, is much milder, suggests that walking plays an important role in the delay of complications, since the level of autonomy of the patients belonging to this group is much greater and in turn has a longer life expectancy, until late adulthood. The actual hypothesis is reinforced by recent studies in mice, which reveal that regular exercise could extend the life expectancy of Type II SMA by $50 \%[15]$.

Duchenne muscular dystrophy (DMD) is a genetic disorder characterized by progressive muscle degeneration and weakness. DMD is the most com- 
mon hereditary muscular disease, with an estimated incidence of 1 per 3,500 male born. It is one of nine types of muscular dystrophy[16].

DMD is caused by an absence of dystrophin, a protein that helps keep muscle cells intact. Symptom onset is in early childhood, usually between ages 3 and 5. The disease primarily affects boys, but in rare cases it can affect girls.

Muscle weakness can begin as early as age 3, first affecting the muscles of the hips, pelvic area, thighs and shoulders, and later the skeletal (voluntary) muscles in the arms, legs and trunk. The calves often are enlarged. By the early teens, the heart and respiratory muscles also are affected.

- Multiple sclerosis. (MS) Multiple sclerosis is a condition where nerve fibers in the spinal cord become damaged by the immune system (the body's natural defense against infection and illness). The immune system mistakenly attacks a substance called myelin, which surrounds nerve fibers and helps with the transmission of nerve signals. In MS, the myelin around the nerve fibers becomes damaged, which disturbs the messages coming to and from the brain. This can result in paralysis or progressive weakness[17]. In the last 15 years, both the prevalence and the incidence of MS has doubled and already affects 2.3 million people in the world. The prevalence MS varies considerably, from high levels in North America and Europe ( $>100 / 100,000$ inhabitants) to low rates in Eastern Asia and sub-Saharan Africa (2/100,000 population) [18].

- Stroke. Stroke or cerebrovascular accident occurs when the blood supply to the brain is disturbed; brain cells die which can lead to brain damage that often results in paralysis[19]. The worldwide impact of stroke has a prevalence of roughly $3 \%$ of the adult population[20]. About 1 million strokes (or CVA) occur every year in the European region (5 million in the world). Around $25 \%$ of men and $20 \%$ of women who reach 85 years of age is expected to suffer a stroke. A $27 \%$ increase in the incidence of stroke is predicted until 2025. Although age is the most important risk factor to suffer a stroke, the trend follows an increase in stroke cases at younger ages. In 2050, the average age to suffer a stroke will be 47.7 years[21]. 
In addition to representing almost $10 \%$ of all annual deaths, stroke is a major cause of long-term disability for those survivors. It affects almost all the human functions of patients, which makes it difficult to perform simple daily activities. $1.8 \%$ of people in Europe are survivor of a stroke and $72 \%$ of those affected suffer weakness in the legs, which affects walking.

- Cerebral palsy.(CP) Cerebral palsy describes a group of psychomotor developmental disorders, which cause a limitation of the person's activity, attributed to problems in the brain development of the fetus or child. Psychomotor disorders of cerebral palsy are often accompanied by sensory, cognitive, communication and perception problems, and in some cases, behavioral disorders. The incidence of this condition in developed countries is approximately $2-2.5$ per thousand births. This incidence has not declined in the last 60 years despite medical advances such as monitoring the vital signs of fetuses. Cerebral palsy involves loss of movement and balance through injury to the cerebral cortex that affect the motor cortex[22]. Cerebral palsy has no known cure; Medical intervention appears as an aid. These treatments for the personal development of the patient are introduced into their daily life throughout life. Some studies [23], [24] demonstrate that a repetitive gait training has important improvements in patients with this disease. In the last 40 years the prevalence of CP has risen to well above 2.0 per 1000 life births[25].

- Spinal cord injury. (SCI) The spinal cord transmits signals to and from the brain and body. If the spine is injured, the brain may not be able to transmit signal to the muscles, causing paralysis. The higher up the spine the injury occurs, the worse the paralysis will be. $52 \%$ are paraplegic and used to have trunk mobility, $48 \%$ are quadriplegic.[26]. SCI results in disturbances to normal sensory, motor, or autonomic function and ultimately impacts a patient's physical, psychological, and social well-being. It is estimated a global incidence of 40 to 80 cases per million population[27].

The lack of mobility derives in side effects, most of them associated to the permanent sitting posture and the own lack of movement of the limbs. The main 


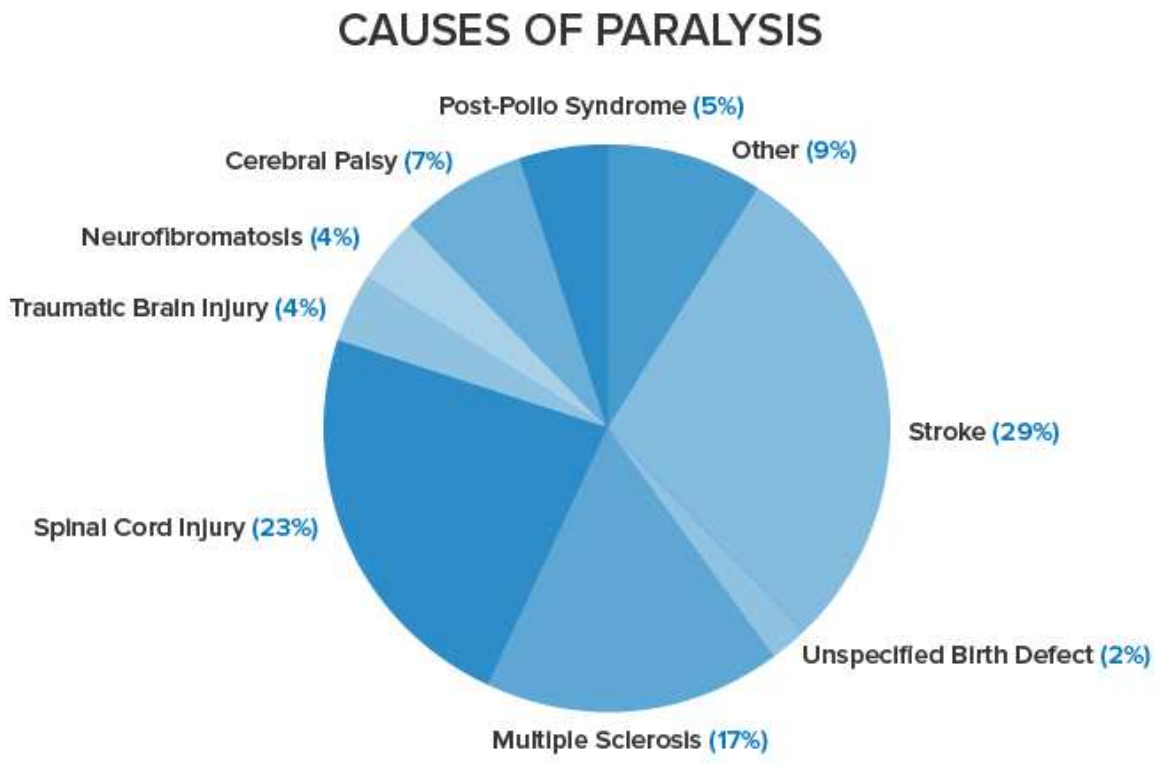

Figure 1.1: Most common causes of paralysis.[1]

physiological effects due to the inability to stand and walk are muscular atrophy, loss of bone density, skin problems and ulcers, increased incidence of urinary tract infection, muscle spasm and spasticity, impaired lymphatic and vascular circulation, impaired digestive operation, and reduced respiratory and cardiovascular capacities. Depression, pain, job loss and poor active sex life are the most significant psychological and psychosocial collateral problems of gait impairment.

To avoid the above problems of the lack of mobility or in some cases to improve the capacity of motion of the user's limb, rehabilitation techniques execute specific motion in the limb. The limb movement improves blood flow, which avoids some of the problems mentioned above. If in addition, rehabilitation movements are performed keeping the person upright, the effects of the lack of mobility are reduced. Analyzing the diseases discussed above, gait rehabitation is applied for stroke, SCI and PC while gait trainning is applied as a therapy to conteract the side effects of chronic deseases and to improve the quality of life of NMDs, MS. Nowadays, the most effective method of gait rehabilitation is body-weight supported treadmill training[28]. Patients who suffer stroke or incomplete SCI gained functional walking ability after this type of rehabilitation. In some types of diseases such as stroke, 
incomplete spinal cord injury or $\mathrm{CP}$, the repeated motion of walking helps in the formation of new neural pathways with which the users relearn to walk or improves their ability of walking[29, 30, 31] .

But this treadmill training has to be done by two or more therapists that manually move the user's leg and stabilize the torso. This is a major constraint to maximize the benefit that this type of rehabilitation may involve in patients with lack of mobility, because it is the trainer's physical capacity which limits the performance of rehabilitation and not the need of the patient.

In response to this need, a new branch of research in assistive robotics is being developed to automate physiotherapy, called rehabilitation robotics. From this perspective the work of the therapist will be to oversee the work of the assistive robot, while the robot is the one that performs the physical work with the patient. In more advanced developments the physiotherapist can teach the robot to perform movements and once learned the robot repeats the movements the number of times necessary.

\subsection{Robotic gait exoskeletons}

A robotic gait exoskeleton, a kind of rehabilitation robot, is an integrated system that unites robot's strength with the cognition of the human wearer. These developments are devised to increase the locomotive ability of an individual who is suffering from leg impairment, which can be paralysis or muscle weakness.

Currently, exoskeletons can be classified into three groups according to their function: force augmentation exoskeletons, treadmill rehabilitation exoskeletons and wearable exoskeletons.

1. Force augmentation exoskeletons The early developments of exoskeletons were in the military field to provide soldiers with extra strength, especially to carry a heavy backpack load for long distances. These types of exoskeletons can be used by both healthy people to increase their capacity in both the military and the industrial field, or by people who have lost strength any limbs, due to a disease that causes muscle weakness or elderly. These devices sense the user's residual force and amplify it. Some of the most representative exoskeletons in this category are shown in Figure 1.2 and 1.3 . 
- HULC (Human Universal Load Carrier), a hydraulic exoskeleton to enhance mobility and increase endurance of soldiers, commercialized by Lockheed Martin is an evolution of BLEEX (Berkeley Lower Extremity Exoskeleton) a development of the university of Berkeley[32. 33]. HULC was proved to augment the strength of its wearers, allowing them to lift 200 pounds without impediment. HULC also lowered the wearer's metabolic cost, meaning soldiers could march with a load while having a decreased oxygen consumption and heart rate. HULC estimates the human motion by reading the dynamic state of the exoskeleton [33].

- XOS-2 is the evolution of the Sarcos exoskeleton promoted by Raytheon, a corporation specializing in defense. Released in 2010, the hydraulic actuated, XOS-2 allows users to lift and carry heavy objects for long periods with little effort. XOS-2 suit utilizes force sensor to implement a get-out-of-the-way control scheme[34].

- NTU-LEE (Lower Extremity Exoskeleton of Nanyang Technological University) as a research prototype, this electrical exoskeleton has similar function as HULC and XOS-2 but different control method. NTULEE exoskeleton triggers the motion with a Zero Moment Point (ZMP) method estimating the user's intention of balancing [35].

- HAL-5 (Hybrid Assitive Limb from the University of Tsukuba). Nowadays, CYBERDYNE company performs the marketing and distribution of the HAL 5 electrical robotic exoskeleton suit, realeased in 2011. In the first design of HAL-5, the user intention was read by using EMG signal, later they employ user's plantar pressure sensing[36].

HULC, XOS-2 and NTU-LEE were developed to walk long distances carrying a heavy load. These systems help soldiers, fire fighters or other emergency personnel to carry mayor loads with minimal effort. In contrast, HAL5 was thought to improve the performance of a user who has lost strength in the limbs.

These types of exoskeleton are werable, carrying their own power supply, so energy autonomy is taken into account in the design process. HULC can 


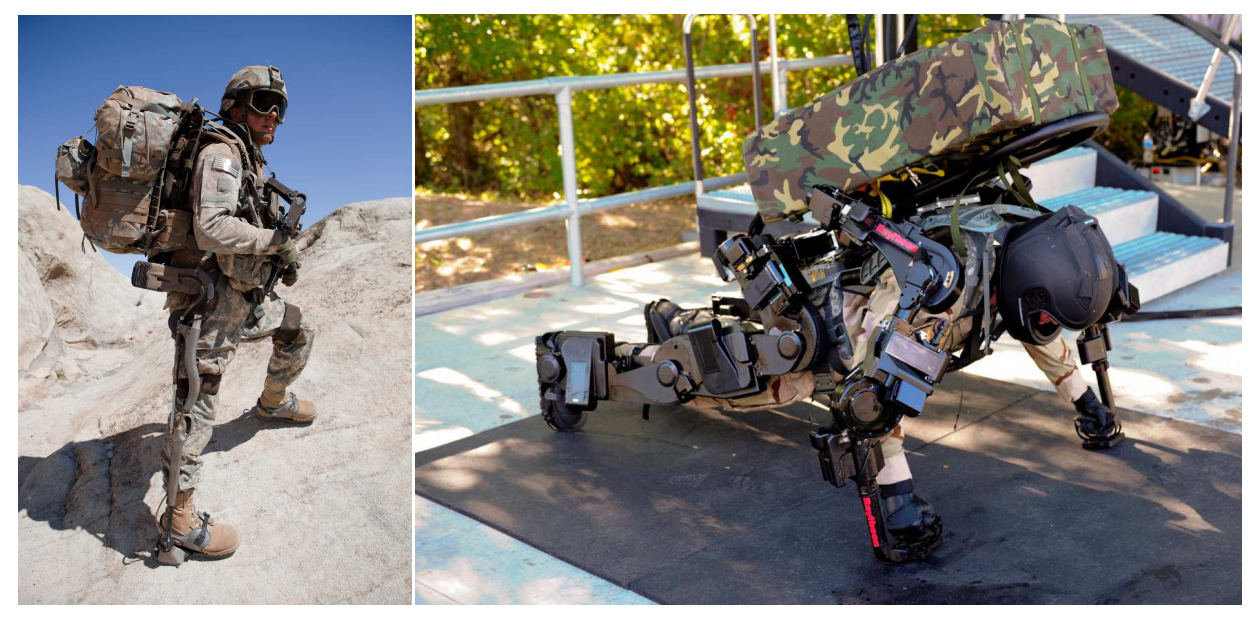

Figure 1.2: Force amplification exoskeleton, HULC, XOS-2.

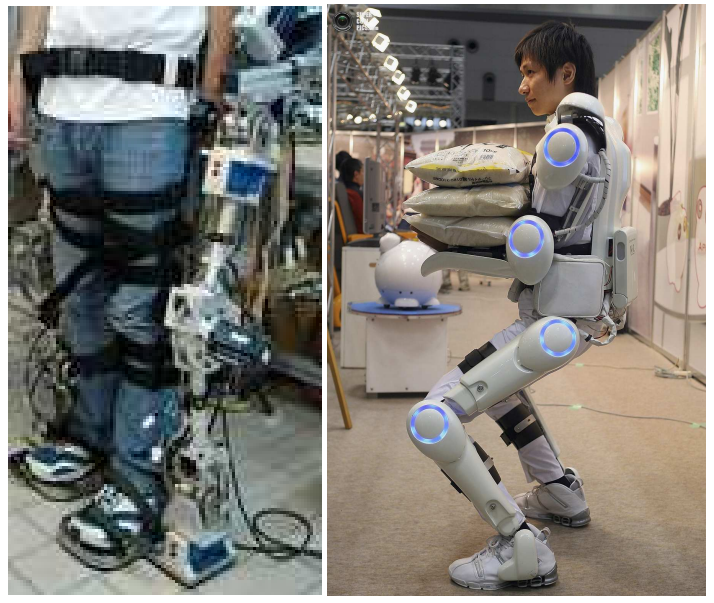

Figure 1.3: Force amplification exoskeleton, NTU-LEE and HAL-5. 
reach twelve hours working in a mission, while XOS 2 provides endurance of about eight hours.

\section{Body weight support rehabilitation systems}

Classical rehabilitation therapy is based on a body weight support (BWS) system in which various therapists reproduce the motion of the user limbs. To avoid this difficult and laborious work, these rehabilitation robots are also BWS systems that move the limbs of the patient based on the therapist's experience. In the case of patients with neurological diseases, such as stroke or cerebral palsy, a gait rehabilitation exoskeleton can help the user to relearn and recover the motion of their limbs. These repetitive motions, that the rehabilitation robot does, help in the formation of user new neural path-ways to relearn to walk for those diseases that are able to be rehabilitated (stroke, SCI and CP)[28]. Other benefits of these rehabilitation robots are related to the upright posture and the motion itself that include an improvement in circulatory and excretory systems.

(a) Gravity Balancing Leg Orthosis (GBO)

In the University of Delaware a passive orthotic device called, Gravity Balancing Leg Orthosis (GBO) [37] was developed. This orthosis varies the gravity that the patient feels, changing the elastic parameters of the springs that hold the user.

(b) Lokomat

It is one of the most popular assisted rehabilitation robot that consists on a BWS system that provides motion to the patient lower limbs on a treadmill. Lokomat[38] by Hocoma (see Figure 1.4) only provides motion in the sagittal plane of hip and knee. A kinematic trajectory can be defined at the foot for each patient but the reduced number of degrees of freedom mentioned produces differences in the kinematic performance compared to human gait. The complete device resides on a large parallelogram which is counterbalanced by a passive spring. The pretension in the spring is adjusted so that the weight of the Lokomat is compensated for, limiting upward or downward external forces to 
the subject during training[39]. In the first vertions, the DC actuators of Lokomat made the motion of the joint too much rigid to allow the patient to exert part of the force in the motion. In the last release, the joints incorporate force sensors to allow the user to perform a certain amount of force when walking.

(c) LOwer extremity Powered ExoSkeleton (LOPES)

The treatmill based device, called LOPES[40] by the University of Twente in Netherland (shown in Figure 1.4), combines a freely translatable and 2-D-actuated pelvis segment with a leg exoskeleton containing three actuated rotational joints: two at the hip and one at the knee. The joints are impedance controlled to allow bidirectional mechanical interaction between the robot and the training subject. Evaluation measurements show that the device allows both a "patient-in-charge" and "robot-in-charge" mode, in which the robot is controlled either to follow or to guide a patient, respectively. The impedance-force control and actuators design of the LOPES exoskeleton allow improvements on active participation and task specificity, achieving a broader rehabilitation concept.

(d) Active Leg Exoskeleton (ALEX)

Active Leg Exoskeleton (ALEX[41]) by University of Delaware (see Figure 1.5) offers an improved and more effective gait training allowing interaction forces between the subject and the orthosis. With an arquitechture similar to the Gravity Balancing Leg Orthosis[37] GBO, an harness on the thunk keeps the user stability. The assistance can vary to make it completely rigid, where the user cannot contribute to the motion, or with almost zero force if the user is able to provide the needed force. These features and the wide range of joint mobility enhance the quality of the rehabilitation training.

(e) Walktrainer

Developed in the Swiss Foundation for Cyberthosis (SFC), Walktrainer[42] (Figure 1.5) is an overground, BWS, rehabilitation device which includes a pelvic and two legs orthosis. It is designed to let robotic re- 
habilitation and electrostimulation. Overground therapy offers a more natural support to the user and the sensation of real motion.

The table 1.1 shows a comparison of the main BWS exoskeletons attending to their Degrees of Freedom (DOF).

\begin{tabular}{|c|cccc|}
\hline & \multicolumn{4}{|c|}{ Actuated Degrees Of Freedom } \\
& Pelvis & Hip & Knee & Ankle \\
\hline Lokomat & N/A & 1 & 1 & N/A \\
\hline LOPES & N/A & 2 & 1 & N/A \\
\hline ALEX & 3 & 2 & 1 & 1 \\
\hline WalkTrainer & N/A & 3 & 1 & 3 \\
\hline
\end{tabular}

Table 1.1: Active degrees of freedom of rehabilitation BWS exosketelons.

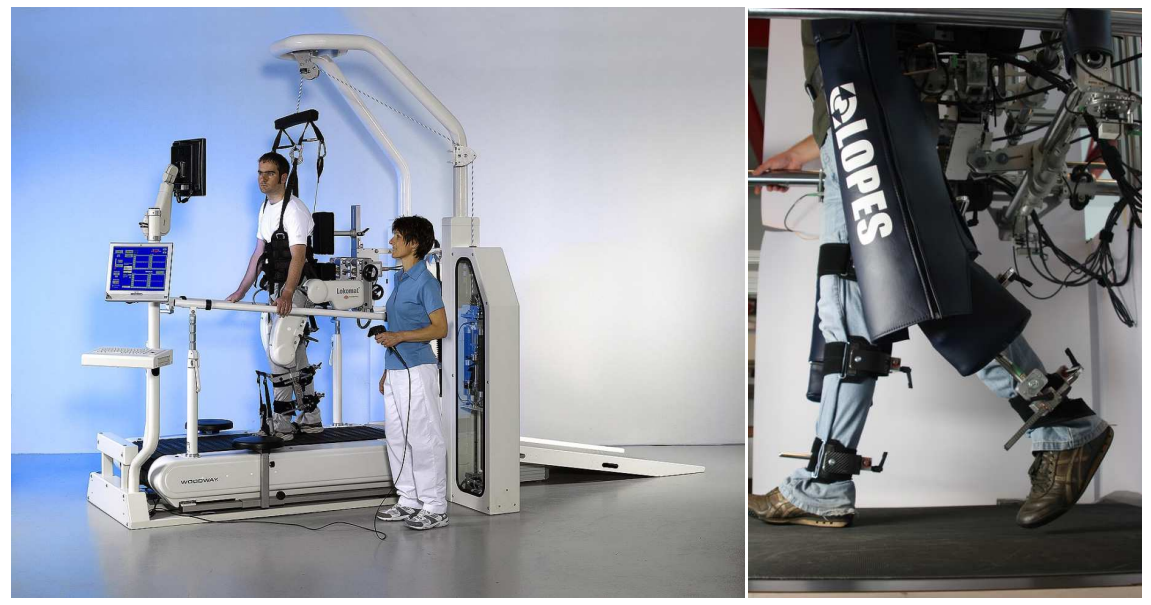

Figure 1.4: Representative BWS robotics rehabilitation: Lokomat by Hocoma (left) and LOPES by the University of Twente (right).

The next step in the evolution of gait rehabilitation robotics are wearable and autonomous devices that the therapist can use on the medical center and that the user can take home. Then, the duration of the therapy session can be extended, improving the rehabilitation therapy and the user can be assisted by the exoskeleton in daily life activities. As it is presented in Section 1.2, some diseases such as NMDs, MS or some type of SCI, are chronic diseases that 


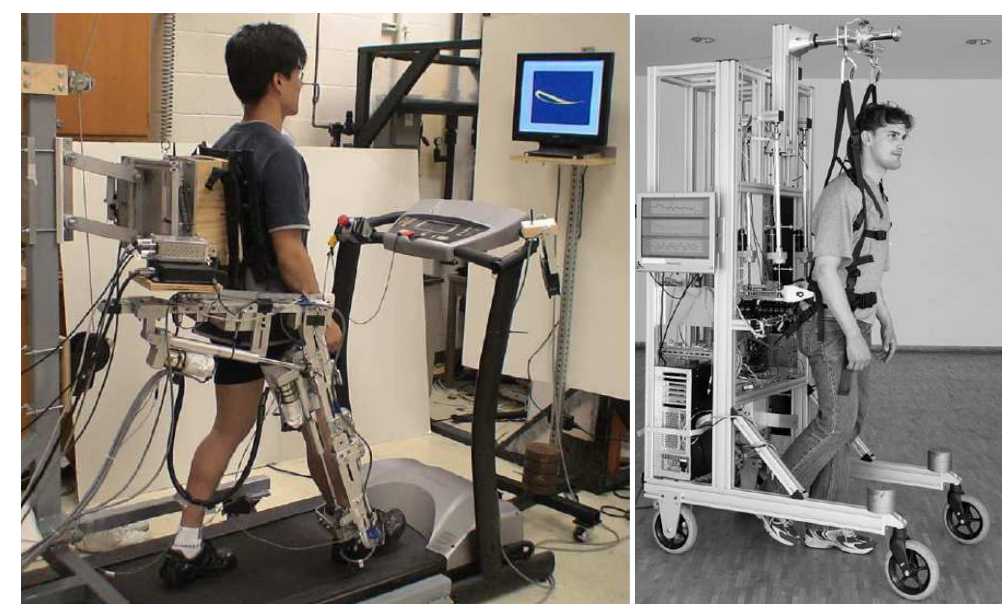

Figure 1.5: Rehabilitation robot ALEX by University of Delaware (left) and Walktrainer by Swiss Foundation (right).

need technical assistance to mobility; therefore, this concept of exoskeleton also can be used as technical aids for these diseases.

\section{Wearable exoskeletons or active orthoses}

The rehabilitation of patients would substantially improve if the user could take the exoskeleton home. Besides, this is completely essential in the patients with chronic deseases who permanently needs technical assistance to mobility. And it would be possible to estimate the evolution and improvement of its medium and long-term use. A further step would be that the exoskeleton could be used outdoors with sufficient autonomy to be used in the daily routine of the user. This case would include the benefits of rehabilitation and mobility aid, allow the user to navigate and interact upright with both physiological and psychological benefits that this would entail.

The main feature of these devices is to be portable and autonomous. These autonomous and portable assistance exoskeletons are commonly called active orthoses [43]. Today, there are commercially available autonomous lower-limb orthotic devices for paraplegic patients. These devices were developed respectively by Argo Medical Technologies (ReWalk [44]), Rex Bionics (REX [45]) and Ekso Bionics [46], whose devices are shown in Figure 1.6. 


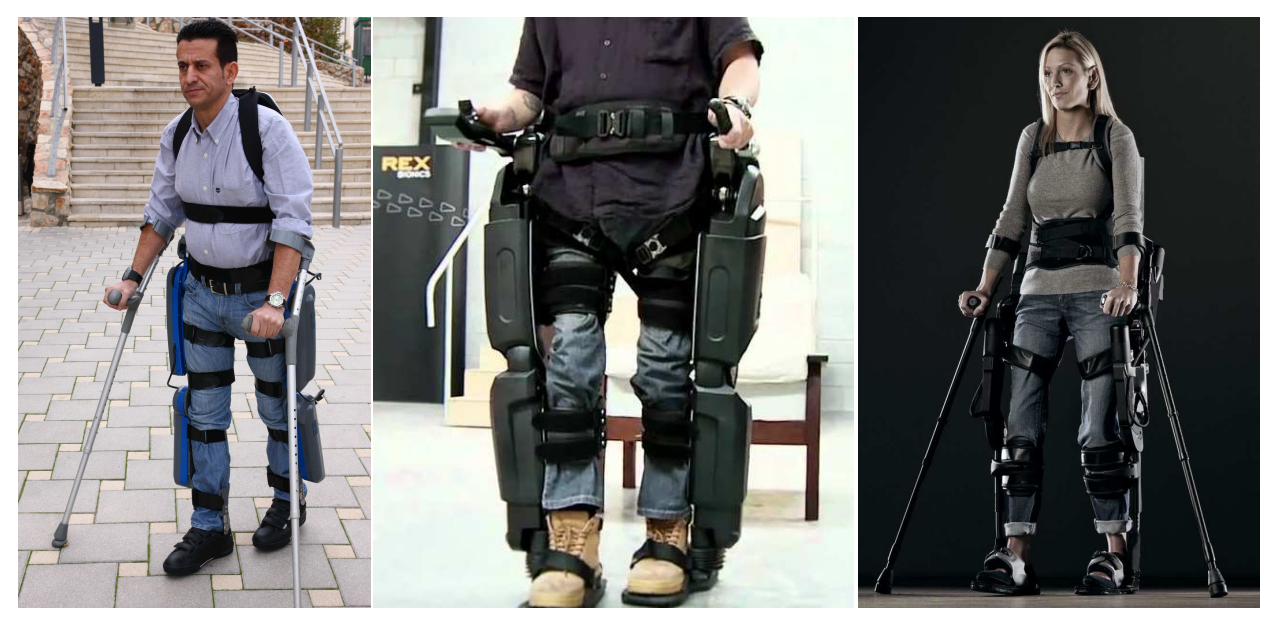

Figure 1.6: Active othoses, Rewalk, REX and Ekso.

\section{Rewalk}

Rewalk is a robotic active orthosis designed to help paralyzed people to walk. It only has two actuated degrees of freedom by DC motors at the knee and the hip while the ankle joint is not actuated.

\section{Ekso}

At the same time, from a development of the Berkeley University, Ekso bionics offers a device with the flexion and extension of hip and knee actuated by hydraulic and also include elastics elements with high rigidity in the hip abduction and adduction and ankle dorsi-plantar flexion[46].

In both devises, Rewalk and Ekso, the user provides stability to the usermachine system with the help of clutches.

3. $\operatorname{Rex}$

The REX device does not need the use of crutches, providing static stability, but with an unacceptable walking speed $(0.05 \mathrm{~m} / \mathrm{s})$ [45].

\section{Indego}

Some research devices became into commercial orthoses, such as, the Vanderbilt Orthosis [28], that now is commercialized by Parker with the name of Indego. Indego helps people with mobility impairments to walk and participate 
in over-ground gait training. Indego has a modular design to help the user to don/doff. It is lightweight with only 11.8 kilograms and it has two hours of autonomy. Indego has been designed as a tool for therapy and for personal mobility for people with spinal cord injuries. This devices have two active DOF at hip and knee flexion.

\section{Mina}

Also Mina[47] research device by the IHMC (Florida Institute for Human Machine Cognition), was taken out of the university by the NASA Johnson Space Center. This orthosis is designed to provide mobility assistance for rehabilitation and training. The length of the limbs is adaptable to each user. The robot features two active degree of freedom at the hip flexion/extension and knee flexion/extension with torque controllable actuators and passive joints to allow for hip ab/adduction and hip internal/external rotation. The powered joints, based on Series-Elastics Actuators (SEA), will be capable of variable impedance, ranging from zero impedance for transparent mode to high impedance for stiff position control. But this variable impedance is implemented electronically. As we will see along this thesis the use of intrinsical impedance provides safety, economy and more natural walking.

In any case, the development of autonomous, portable lower limb active orthosis will contribute to providing physically challenged persons with a physical therapy and also:

(1) Improved quality of life, making their daily life much more "normal" than ever.

(2) Autonomy and endurance to move around.

(3) Details which are relevant for the disabled: normal height at standing up posture, allowing the person to talk with people at their same height, and to reach switches and objects which were placed at the height of an upright person.

Table 1.2 depicts a comparative between these commercial orthosis, none of them deal with a reduction in energy consumption. Only Ekso active orthosis walks with moderately adequate autonomy (three hours), a lightweight structure and a natural user-intention monitoring system. 


\begin{tabular}{|c|c|c|ccc|c|c|}
\hline & Weight & Autonomy & \multicolumn{2}{|c|}{ Degrees Of Freedom } & Crutches & Speed \\
& & (Hour) & Hip & Knee & Ankle & needed & m/sec \\
\hline Rewalk & $22 \mathrm{~kg}$ & 2.5 & $2(1 \mathrm{~A} 1 \mathrm{P})$ & $1 \mathrm{~A}$ & $1 \mathrm{P}$ & Yes & 0.83 \\
\hline REX & $39 \mathrm{~kg}$ & 2 & $2 \mathrm{~A}$ & $1 \mathrm{~A}$ & $2 \mathrm{~A}$ & No & 0.05 \\
\hline Ekso & $20 \mathrm{~kg}$ & 3 & $2(1 \mathrm{~A} 1 \mathrm{P})$ & $1 \mathrm{~A}$ & $1 \mathrm{P}$ & Yes & 0.89 \\
\hline Indego & $11.8 \mathrm{~kg}$ & 2 & $1 \mathrm{~A}$ & $1 \mathrm{~A}$ & $1 \mathrm{P}$ & Yes & 0.45 \\
\hline Mina & $14 \mathrm{~kg}$ & 2 & $1 \mathrm{~A}$ & $1 \mathrm{~A}$ & $1 \mathrm{P}$ & Yes & 0.20 \\
\hline ATLAS & $10.8 \mathrm{~kg}$ & 2.5 & $1 \mathrm{~A}$ & $1 \mathrm{~A}$ & $1 \mathrm{~A}$ & Yes & 0.88 \\
\hline
\end{tabular}

Table 1.2: Main features of commercial active orthoses and ATLAS orthosis. In the field of degrees of freedom it is used A to Active and P to Pasive. (Data for the year 2016.

Analizing the data of the Table 1.2 and 2.1, currently, the runtime of commercial active orthosis is far from being enough for a daily use. It is noted that this limited autonomy of the active orthoses also limits a better marketing and distribution due to the low battery life and high energy consumption.

Due to the low energy density of current batteries, it is necessary to reduce the energy consumption of active orthoses, to achieve acceptable autonomy. Being autonomy such an important feature, several research groups have investigated energy consumption both in powered orthoses as well as in individual joints. In Valiente and Walsh's researches [48, 49], force amplifying exoskeletons are designed to help to carry payloads. The aim is to reduce the energy consumption of the user while carrying a payload with an under-actuated exoskeleton. Figure 1.7 shows both authors wearing the under-actuated exoskeleton.

In Walsh's work a force controllable actuator based on series elastic actuation concept (SEA) is used at the hip in an attempt of exploiting the conversion of potential energy into kinetic energy. A variable-damper mechanism was installed at the knee to lock it and support the body weight in the early stance. A passive spring was used in the ankle to store energy during early stance and release it in the push off phase at the end of stance.

In Valiente's thesis[50], similar strategies are applied, with a variable-damper at the knee and passive ankle springs. In the hip, passive springs are used instead 


\subsection{Thesis objective}

of the force controllable actuator.

None of these theses achieved completely their goal of energy reduction of the user oxygen consumption. This is due to the weight and inertia that the exoskeleton itself adds and also to the high intrinsic stiffness of the joint that does not allow to exploit the intrinsic dynamics of the body limbs.

Although in the case of these exoskeletons the goal is to reduce the energy consumption of the users and not the exoskeleton itself, these economic perspectives in the design of exoskeletons inspire the background of this thesis.
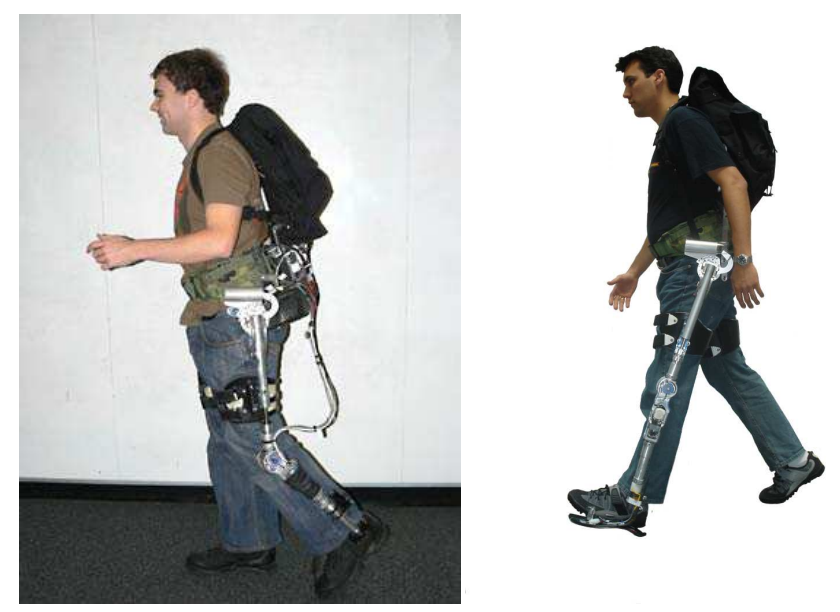

Figure 1.7: Under-actuated exoskeleton from MIT.

\subsection{Thesis objective}

The goal of reducing energy consumption in gait exoskeletons is approached in this Thesis, as it is a problem not solved yet, with relevant implications. The healthy human gait is extremely energy efficient. Being this true, it is still very difficult to generate an artificial system with comparable efficiency and versatility using current knowledge and technology. Significant hurdles and challenges persist both from the theoretical and practical point of views. The objective of this thesis is to study the problem of energy expenditure and its management applied to active orthoses in order to reduce energy consumption, thus increasing their autonomy while improving performance. This would lead to a wider use, better marketing 
and distribution of these devices. The final aim of this project is to help impaired people to walk with improved autonomy and performance, providing a daily-life assistance exoskeleton. This goal of reducing energy consumption in this thesis is done from a holistic point of view, that is, taking into account all the characteristics that relate to this energy consumption.

To achieve that goal this thesis presents and analizes two models of energetics of human gait:

1. Biomechanics human gait model. A simplified model of human gait is presented. The kinetics and dynamics of the complete leg and of the hip, knee and ankle joints are analyzed. This model includes the main characteristics that make the human gait so efficient.

2. Robotic gait model. Two paradigms of bipedal robots, Zero Moment Point (ZMP) and dynamic walking are analyzed. The ZMP approach features very versatile bipeds, able to walk, climb and descend stairs, sit and stand up and even kick a ball, but with a high energy consumption. The dynamic walking approach in contrast to the ZMP walks on even level terrain with a very low energy consumption.

The human gait model will be used as a reference to draw the strategies on reduction of energy consumption of an extremely efficient model. While the robotic model will show us the state of the art of the tools to be able to perform, analyze and implement the strategies previously studied. This double approach has been established because active orthoses are robotic devices but coupled to human limbs. Human gait and the morphology of its limbs provide a reference when developing energy efficient bipedal robots. On the other hand, robotics offers a deeper understanding in bipedal walking and presents the problems and challenges that a biped walking machine faces with nowadays technology.

\subsection{ATLAS active orthosis as a test-bed for improved energy consumption exoskeletons}

In line with the above mentioned active orthoses and as a test-bed for the evaluation of energy consumption our group in the center for automation and robotics (CAR) 
has designed and developed the ATLAS active orthosis[51] (see Figure 1.8) in the frame of the project DPI12010-18702. Table 1.2 shows some basic features and a deep description can be found in Appendix A.

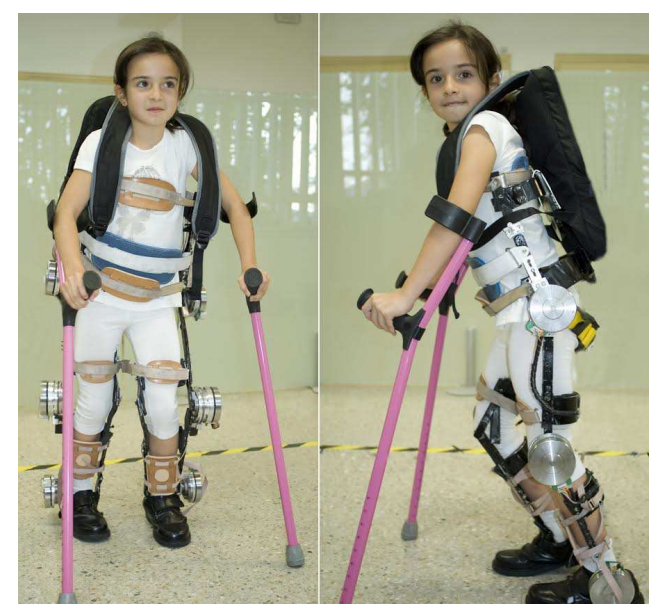

Figure 1.8: The ATLAS active orthosis prototype

This first prototype of ATLAS active orthosis represents what we will call along this thesis first generation active orthosis with the following characteristics:

- Use position patterns usually obtained from clinical gait analyses (CGA) of healthy people or another fix trajectory, such us parameterized trajectories.

- The following of these trajectories is done with rigid actuators, composed of a motor and gearbox with high reduction ratio(above 100:1).

- It does not include passive elements.

- Features high power consumption compared to human expenditure.

Therefore, all the concepts defined in this thesis in order to reduce energy consumption have been utterly implemented in this orthosis and the energy consumption has been measured to have quantitative data on the suitability of the approach. 


\subsection{Thesis outline}

This thesis is schedulled as follows: first, it is discussed in depth how humans walk and the main characteristics of this gait are obtained, both structural and morphological. Its features make this form of bipedal walking the most economical in nature. In chapter 2, human gait is disaggregated to discover the most important features that make it so efficient.

Subsequently, the evolution of bipedal robot developments and their lower energy consumption approaches are analyzed in chapter 3. It is proved that the most efficient bipedal robot walkers reach the efficiency of human walking, but they lack versatility. A compromise between the energy economy and versatility must be found. In this chapter it is demonstrated that the most economic approach in bipedal walking robots is achieved though underactuated devices and a smooth gait control system that avoids impacts.

Based on this double human and robotics track, a bioinspired framework is proposed with some key features to achieve economic modes of walking. This framework is analyzed in a theoretical way throughout chapter 4.

Departing from this analysis a number of key features to be incorporated to the active orthosis in order to improve energy efficiency are deduced:

\section{Kinematic approaches to energy effciency}

(a) Exploiting joint synergies, to transfer energy between joints through bi-articular linkages.

(b) Determine the optimum number of active/passive DOF.

(c) Energy storage and release utilizing elastic mechanisms.

(d) Minimizing actuator power in certain phases of the gait.

\section{Dynamic control approaches to energy effciency}

(a) Impedance control techniques to minimize impact energy losses.

(b) Exploiting intrinsic dynamics

(c) Optimizing mass distribution. 


\subsection{Thesis outline}

Then, an assesment of the proposed approach is carried out through real experiments. The model parameters have to be adapted to the specific characteristics and requirements of the ATLAS active orthosis which is the experimental testbed where the proposed algorithms have been analyzed. It is shown in chapter $\mathbf{5}$ how it is able to take advantage of the synergy between joints while walking, allowing to reduce the number of actuators of the orthosis and therefore the energy consumption. Finally in chapter 6 and 7, the proposed energy saving key features yield to a redesigned and improved ATLAS orthosis.

As a result of applying in ATLAS active orthoses this thesis proposal an important reduction in energy consumption compared to the first generation of orthosis designs is achieved; while providing a more natural gait. This energy based comparison is performed using the ATLAS orthosis as a testbed. The implementation of these techniques allows us to reach a reduction of $40 \%$ in the energy consumed during walking. This will improve signifficantly the autonomy of the wearable active orthoses and so the installation of these active orthoses in the market.

The results of this $\mathrm{PhD}$ thesis has contributed to three patents:

- PCT/ES2015/070855. E. Garcia, M. Cestari, D.Sanz-Merodio, and X. Carrillo "Exoesqueleto para asistencia al movimiento humano" (11-25-2015) Assignee: CSIC-UPM (85\%), Marsi Bionics (15\%).

- ES201330882 and WO2014198979 (Prosecution). M. Cestari, D.Sanz-Merodio, y E. Garcia "Articulation with controllable stiffness and force-measuring device" (06-13-2013). Licensed to Marsi Bionics by the CSIC and UPM.

- ES201330882 - EP2907495 (Prosecution) and US2015265490 (Prosecution). M. Cestari, D.Sanz-Merodio, J.C. Arevalo y E. Garcia. "Walker comprising mechanism for assisting a user in standing and sitting operations" (10-112012). Licensed to Marsi Bionics by the CSIC and UPM.

These three patents have been licenced to Marsi Bionics, spin-off company comercializing ATLAS 2020, the first and only pediatric wearable gait exoskeleton for SMA kids, already installed at the Sant Joan de Déu Children hospital for gait training. 
During the development of this $\mathrm{PhD}$ thesis 8 publications in JCR international journals and 14 conference publications have been produced in several of which mentions and prizes have been obtained. 
Avoid extremes: Be moderate in saving and in spending; An equable and easy gait will win an easy ending.

Robert W. Service

\subsection{Introduction}

From the perspective of reducing the energy consumption of gait exoskeletons, the analysis of human walking is absolutely necessary. Besides showing the kinematic limits and the dynamic characteristics of the legs, it is found that the human way of walking is extremely efficient.

Although it is not a proven conjecture, human walking tends to minimize energy consumption. The energetic cost of human walking increases with speed (see Figure 2.1, , resulting in a series of speeds at which the cost to walk a given distance is optimal (Figure 2.2). In contrast, the cost for a human to run remains constant with speed, so that the cost to run a given distance is speed-invariant (Figure 2.1). The amount of energy used to run a given distance is nearly the same, whether running quickly or slowly [52].

Quadrupeds, in contrast, have optimal speeds within gaits. The cost of locomotion within gait (walk, trot or gallop) increases curvilinearly with velocity. So, there are generally speeds within the gaits of quadrupeds that are less metabolically expensive (see Figure 2.3]3]). 


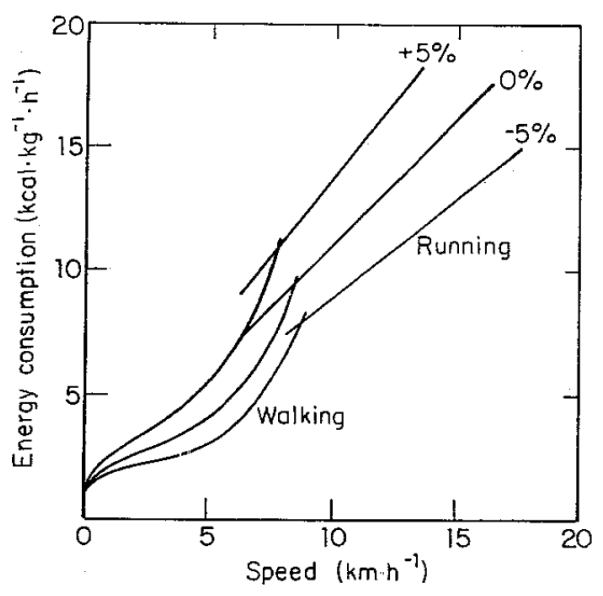

Figure 2.1: Rate of energy consumption versus speed for human walking and running on level $(0 \%)$, uphill $(+5 \%$ gradient $)$ and down hill ( $-5 \%$ gradient).[2]

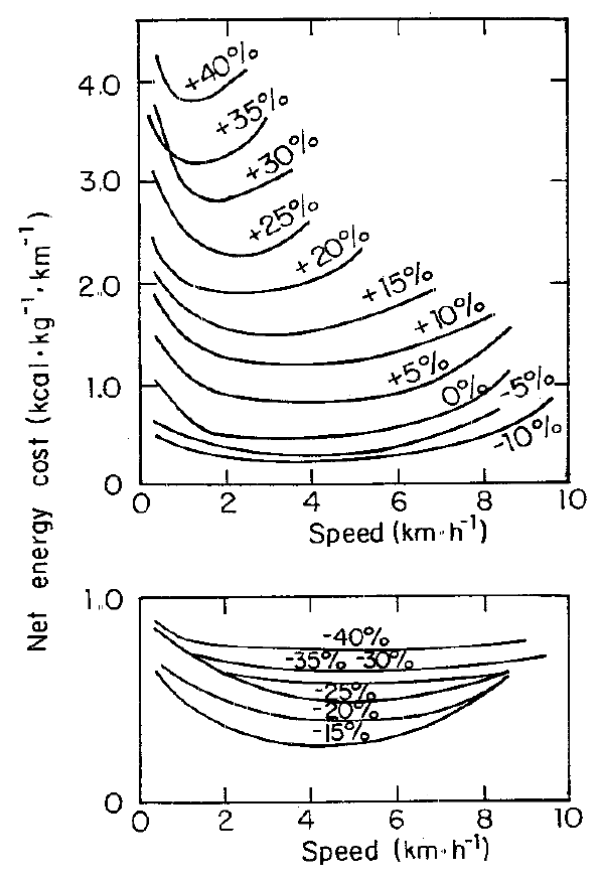

Figure 2.2: Energy consumption per unit of distance in walking at different gradients. For each gradient, a minimum occurs at a given speed.[2] 


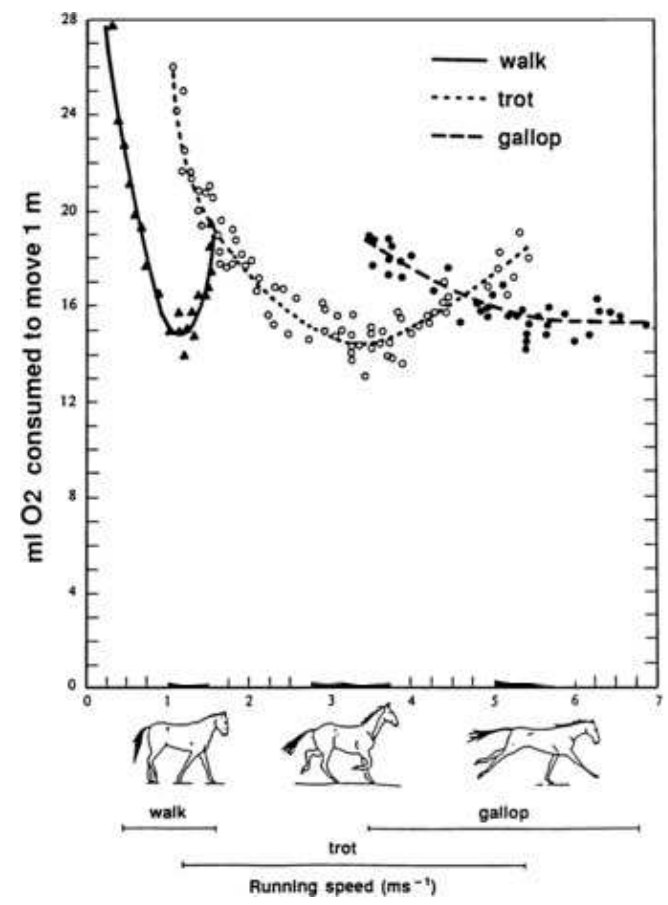

Figure 2.3: Oxygen consumption of a horse running at a range of speeds in three gaits.[3] 
Both humans and quadrupeds share strategies to achieve a form of locomotion versatile and very efficient. Quadrupeds have perfected their mode of locomotion while walking and while running and humans do it while walking.

Quadrupeds use a collision-reduction strategy for reducing energy consumption controlling the foot collision with the ground in their slowest and fastest gaits, walking. On the other hand, while trotting and galloping, quadrupeds use a Spring Loaded Inverted Pendulum (SLIP) mechanism to reduce energy expenditure storing and returning elastic energy[53].

As it is shown in Figure Figure 2.3]3], the energetic cost of transport in quadrupeds has a minimum in all the three gaits. This suggests that at each gait there is a resonant speed at which the effort needed for the oscillation of the leg is minimized[54].

\subsection{Comparison of energy consumption of active or- thoses. Cost of transport COT}

Throughout this thesis the energetic cost of transport (COT) will be used as dimensionless parameter of measurement of energy consumption, which is computed as follows::

$$
\varepsilon=\frac{E}{W L}=\frac{P}{W v}
$$

Defined as the energy consumed to move a unit of weight to a unit of distance, the COT allows to compare different modes of locomotion despite its sources of energy come from different origins.

Table 2.1 shows the cost of transport of the active orthoses presented in Chapter 1. Figure 2.4 [55] compares the cost of transport for humans, animals, vehicles and robots, and it includes the transport cost of the active orthoses presented in the introduction including the weight of the user.

The minimum cost of transport COT of a human when walking is (COT $=0.3$ ) [56].This is a consumption three times lower than any of the presented orthoses. This is understandable because the energy consumption has not been present as a design consideration of these orthoses. 
Table 2.1: Cost of transport of several active orthoses

\begin{tabular}{|l|l|l|l|l|l|}
\hline$\varepsilon_{\text {Rewalk }}$ & $\varepsilon_{\text {REX }}$ & $\varepsilon_{\text {Ekso }}$ & $\varepsilon_{\text {Indego }}$ & $\varepsilon_{\text {Mina }}$ & $\varepsilon_{\text {ATLAS }}$ \\
\hline 1.19 & 19.8 & 0.93 & 2.76 & 6.21 & 1.13 \\
\hline
\end{tabular}

Moreover humans utilize SLIP technique to economize energy while running. Otherwise, humans use a more complex method while walking. This last one combines a set of strategies that includes compliant spring inverted pendulum (SLIP) and collision reduction strategies[57] with ankle push off[4, 58] and curve feet[59]. These strategies are analyzed in the next chapter.

To find the clues to the human superior performance, this chapter analyzes how humans walk, gait kinetics, kinematics, dynamics and muscular structure for gait performance.

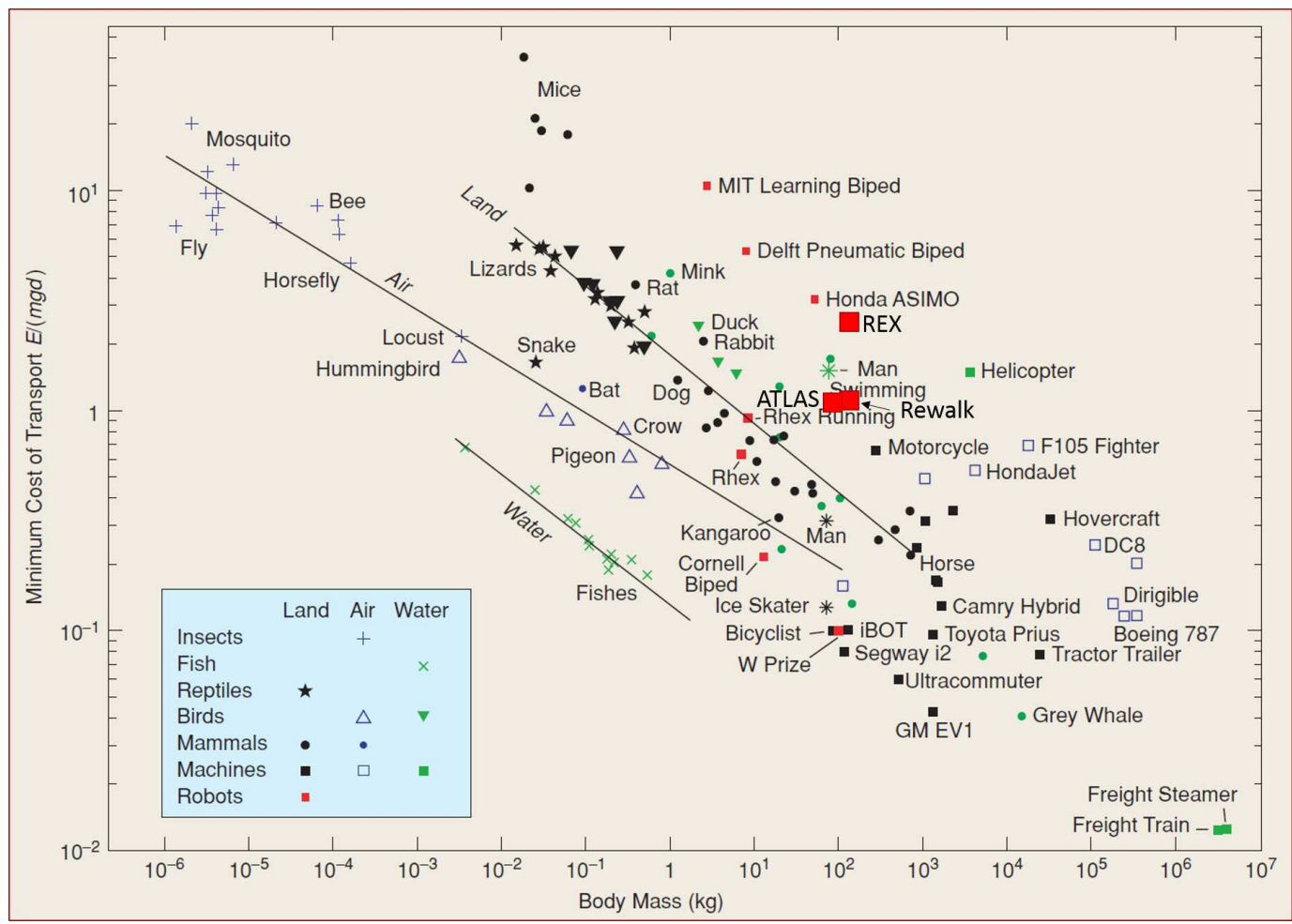

Figure 2.4: Comparison of the cost of transport for humans, animals, vehicles and robots. Also the graph includes the cost of transport of some of the active orthosis.

The key features of human gait discussed in this chapter will be joined with 
Table 2.2: Energy consumption of the leg joints in each anatomical plane (shown in percentages). Computed from the data of [9].

\begin{tabular}{|l|l|l|l|}
\hline Plane of the body & Sagital & Coronal & Horizontal \\
\hline Energy consumption (\%) & $79,25 \%$ & $18,67 \%$ & $2,07 \%$ \\
\hline
\end{tabular}

those obtained from the analysis of designs and dynamics of biped robots. These criteria will be applied to modify the ATLAS orthosis in order to achieve a more energy-efficient active orthosis.

\subsection{Definitions and nomenclature}

Throughout this thesis reference to the biomechanics of humans is continuously made. It is, therefore, necessary to define the specific nomenclature that will be used.

The human leg is a complex kinematic structure composed of a large number of muscles, providing full degrees of mobility. For modeling and control purposes a simplified scheme of the human leg is considered with 7 degrees of freedom: 3 at the hip, one at the knee and three at the ankle. Figure 2.5 defines the different anatomical planes of the body. The movement of the joints in the sagittal plane is referred to as flexion and extension. Motion of the hip in the coronal plane is referred to as abduction (away from the center of the body) and adduction. Moreover, the movement of the ankle in the coronal plane is called eversion when the foot moves away from the human body and inversion when approaching. The degree of freedom remaining in the hip and ankle is called rotation.

Figure 2.6 shows the biomechanics nomenclature of joint direction of motion of hip, knee and ankle that will be used throughout the thesis.

The energy consumption reduction algorithms applied throughout this thesis will be centered on the sagittal plane, since, as it is shown in Table 2.2, it represents $80 \%$ of the total energy consumption. 


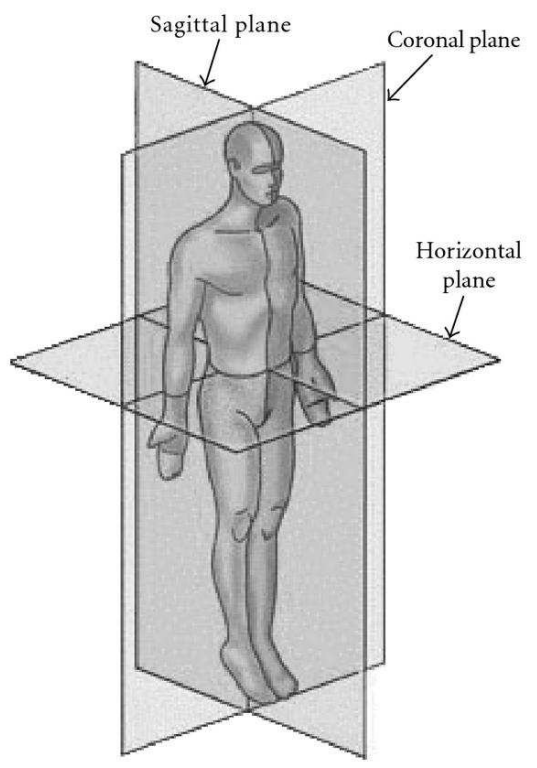

Figure 2.5: Anatomical planes of the body.

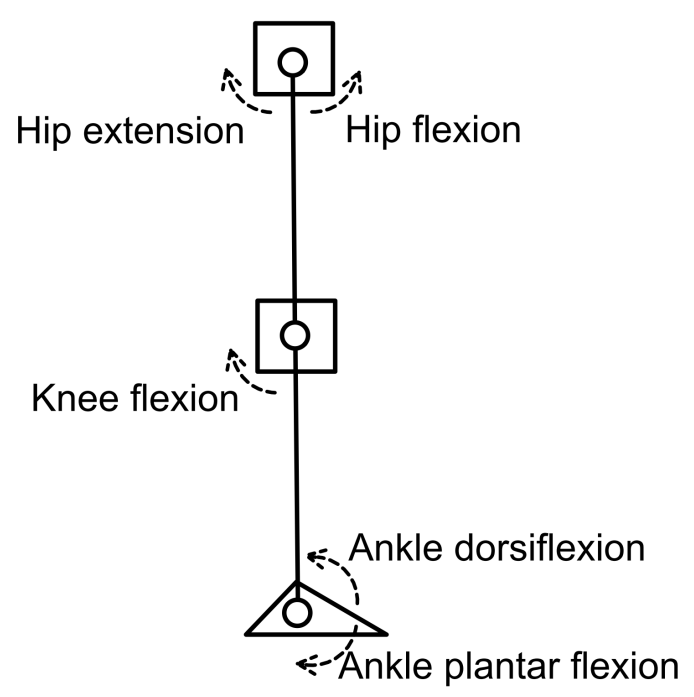

Figure 2.6: Nomenclature of sagittal joint motions. 


\subsection{The human gait cycle}

Human gait is a series of alternating movements of the trunk and limbs which define a forward displacement of the center of mass of the body. Human walking is usually studied as a cyclic process that repeats a pattern over and over. Conventionally, the human gait cycle begins when the foot contacts the ground and ends when the same foot impacts the ground again. For each gait cycle, each leg completes two phases: the stance/support phase is defined as the period of gait cycle when the foot reamins in contact with the ground, while the swing phase is defined as the period of no ground contact. In the gait cycle, the period in which both feet are in contact with the ground simultaneously is defined as the double support phase.

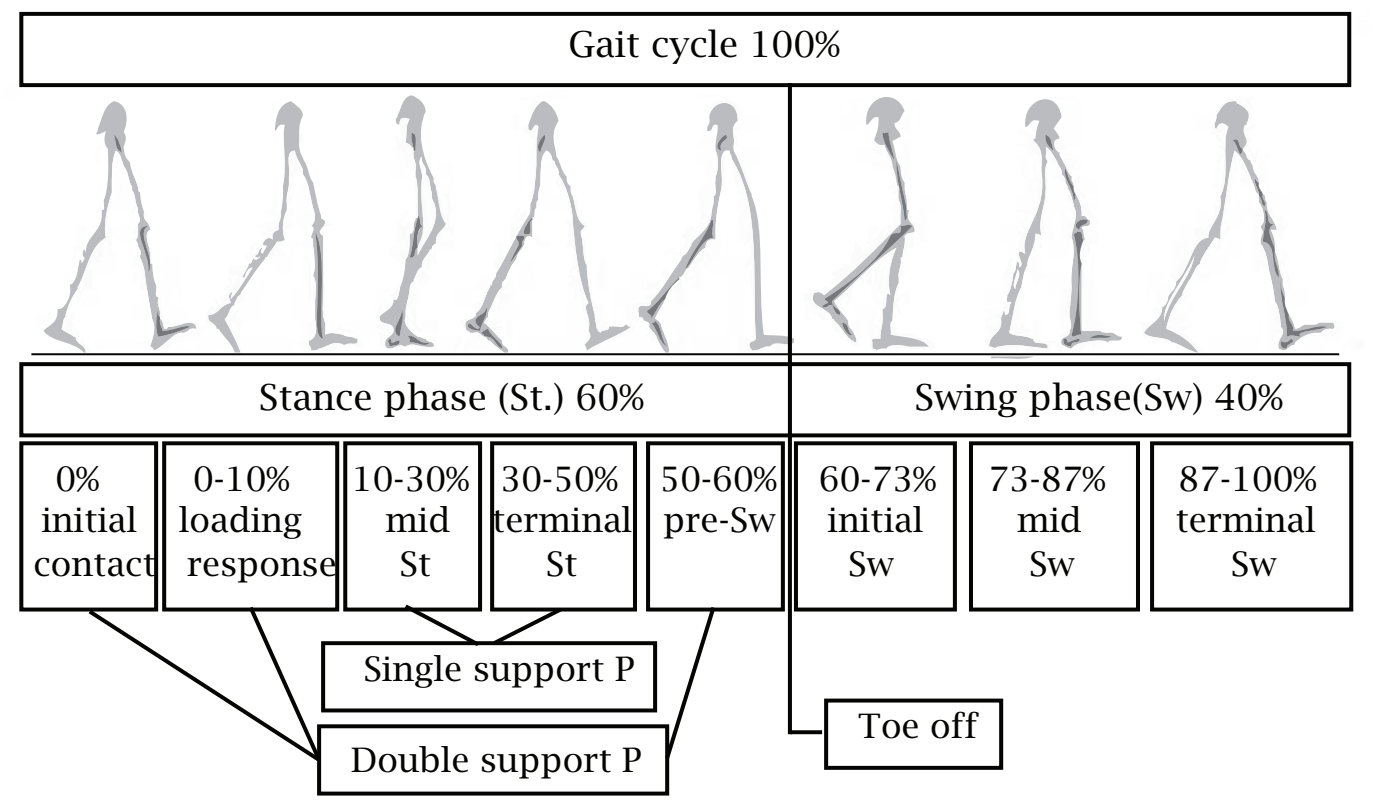

Figure 2.7: Complete gait cycle with detailed phases. St: Stance. Sw: Swing

Figure 2.7 depicts a complete gait cycle, let us analyze each sub phase in more detail[60].

The relevant events in the stance phase are:

1. Initial contact ( $0 \%$ of gait cycle): The heel contacts the ground. Double support phase begins. 


\subsection{The human gait cycle}

2. Loading response ( $0 \%$ to $10 \%$ of gait cycle): In this sub phase the leg absorbs the impact of the body weight. The double support ends.

3. Mid stance ( $10 \%$ to $30 \%$ of gait cycle): Single support begins, the body rolls over the foot like an inverted pendulum, almost without energy expenditure.

4. Terminal support ( $30 \%$ to $50 \%$ of gait cycle): The leg prepares the body motion for the heel strike of the leading leg.

5. Pre-swing ( $50 \%$ to $60 \%$ of gait cycle): Toe-off impulse finishes and the leg lifts off the ground.

In the swing phase the following events occur at the indicated (\%) of the gait cycle:

1. Initial swing (60\% to $73 \%$ ): After the acceleration provided, the foot is lifted to avoid the ground.

2. Mid swing ( $73 \%$ to $87 \%$ ): The leg advances with the knee flexed, like a direct pendulum, preventing stumbling.

3. Terminal swing ( $87 \%$ to $100 \%$ ): The leg is fully stretched and ready for heel strike.

Considering the energy standpoint, each of these phases involves peaks in power consumption for each joint. In the third row of Figure 2.9, maximum and minimum mechanical power requirement events are shown for each joint.

The first positive power peak in the hip motion (H1) is related to the stabilization during the foot contact. The minimum power $(\mathrm{H} 2)$ is required when the body pivots over the leg stretching the quadriceps. The second maximum of power requirement $(\mathrm{H} 3)$ corresponds to the leg forward swing.

In the knee gait cycle five events of mechanical power are shown. In the first (K1), of negative power, the knee brakes the motion after heel strike in the loadresponse phase. In the second event (K2), there is a positive power requirement to stretch the knee after the absorption of the body weight (end of the loading response phase). In event (K3) very little energy is absorbed to maintain the knee straight in the mid stance and terminal support phases. In event (K4) of pre-swing and swing, the power is required to bend the knee. At the end of the mid swing the 
knee is stretched without energy consumption and a little energy (K5) is required in slowing the leg at the end of the swing. It is observed that the knee mainly dissipates energy.

The ankle is essentially the joint that impulses the body in human walking. So, little negative energy is absorbed in event (A1) where it extends during the first four phases of stance of Figure 2.7, while injecting a lot of positive energy (A2) to propel the body forward in the pre-swing phase.

Both in hip and ankle joint alternation between positive and negative phases of mechanical power is observed. Energy accumulates in muscle-tendon units during negative phases and it is released in the next positive power demanding phase.

Since humans tend to minimize energy consumption while walking, through the analysis of human gait, features to generate an efficient gait can be extracted.

\subsection{Strategies and mechanisms to reduce energy ex- penditure in human walking}

Human gait is characterized by a very low amount of power consumption. Sometimes it has been hypothesized that humans walk in a way in which the metabolic energy consumed is minimized [61]. From the design and features of muscles and tendons to the way each joint moves, they all contribute to achieving an agile, adaptable, steady and overall economic gait. They are revised in this section.

\subsubsection{Kinematics of human walking}

Kinematics of human walking describes the direction, extent and speed of the movement of joints and limbs of the body while walking.

First, we will take a look at the motion of the center of mass (CoM) of the body in the sagittal plane while walking, and after that, the motion of each leg joint that produces this body motion will be analyzed. The movement of the upper- body segments (head, trunk, arms...), will not be taken into account. Even though they have an influence on the energetics of the gait (for example, the motion of the arms will help to reduce the impact of the body in the heel strike phase [62]), it will 
not be taken into account in the scope of this analysis for the sake of simplicity of problem formulation.

In the kinematic analysis of the leg joint motion, only the trajectories and velocities of the joint are analyzed. This analysis will be completed in the next section where the dynamics of each joint is integrated to the study.

\subsubsection{Kinematics of the center of mass of the body}

As detailed in the previous chapter, this study is resticted to the sagittal plane, since the energy consumption occurs mainly in this plane. The center of mass excursion on the sagittal plane, walking in level terrain behaves like a sinusoidal curve. The amplitude of the curve is about $5 \mathrm{~cm}$. The maximum height is reached in the middle of the stance phase while the minimum height occurs in the double support phase just after toe off. It is important to remark that this curve is markedly smooth [63], see Figure 2.8

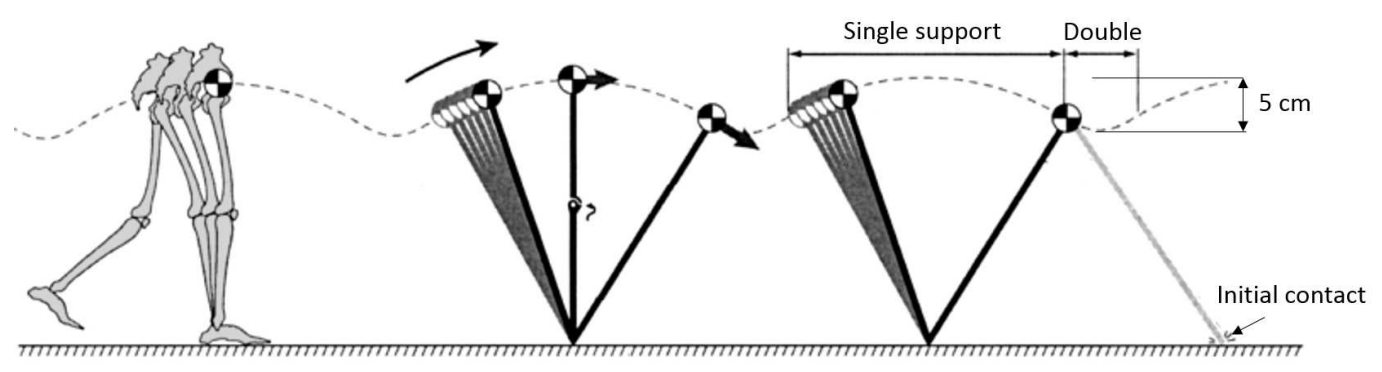

Figure 2.8: The movement of the COM of the body when walking on level ground in the sagittal plane follows a sinusoid.[4]

As will be detailed in chapter 3, though in single support the body behaves like a pendulum, the ankle role in the toe off phase redirects upwards the center of mass of the body. In this manner, a sharper heel strike collision and movement in the center of mass of the body are avoided, that would otherwise bother to the human leg and would increase energy expenditure.

\subsubsection{Kinematics of the leg joints}

The angular trajectory in the sagittal plane of the hip, knee and ankle joint of a healthy human while walking is presented in the first row of Figure 2.9 [5]. The 
main features of these trajectories are:

- Hip - The hip motion in the sagittal plane follows a sinusoidal curve. In support phase the leg is oscillated backwards moving the body forward while in swing phase the leg is moved forward to repeat the cycle. Hip flexion reaches its maximum at the terminal swing. This is followed by a slight extension before heel strike; this allows accommodating the leg to continue the advance steadily. Maximum of hip extension occurs at opposite foot strike.

- Knee - The first flexion of the knee after the heel strike occurs mainly to absorb the impact of the contact and to avoid an excessive vertical translation of the center of mass. Afterwards, the knee is almost blocked to allow inverted pendulum movement of the CoM over the foot. In terminal stance, knee flexion begins providing ground clearance to the swing. In mid swing the knee is extended to prepare for the next step.

- Ankle - In the first double support after heel strike, the ankle stays at $0^{\circ}$. In single stance the ankle continues with a dorsiflexion motion followed by a plantar flexion motion. These movements help the body to rotate over the foot, in single stance. In the second double support the ankle provides a big plantar flexion impulse to redirect de center of mass and provides impulse to begin the swing phase. In swing phase the foot returns to the flat posture to clear the ground.

\subsubsection{Kinetics of human walking}

To have a thorough perspective of how humans try to minimize mechanical energy expenditure while walking, it is not enough to analyze the trajectories of each joint. It is necessary to examine the energetic behavior of each joint, namely the moment, work and power that each joint exerts, this is the kinetics of walking.

Figure 2.9 [5] shows the normalized moment and power of hip, knee and ankle joint in the sagittal plane. Joint moment is normalized to body weight and height; power is normalized to body weight. 

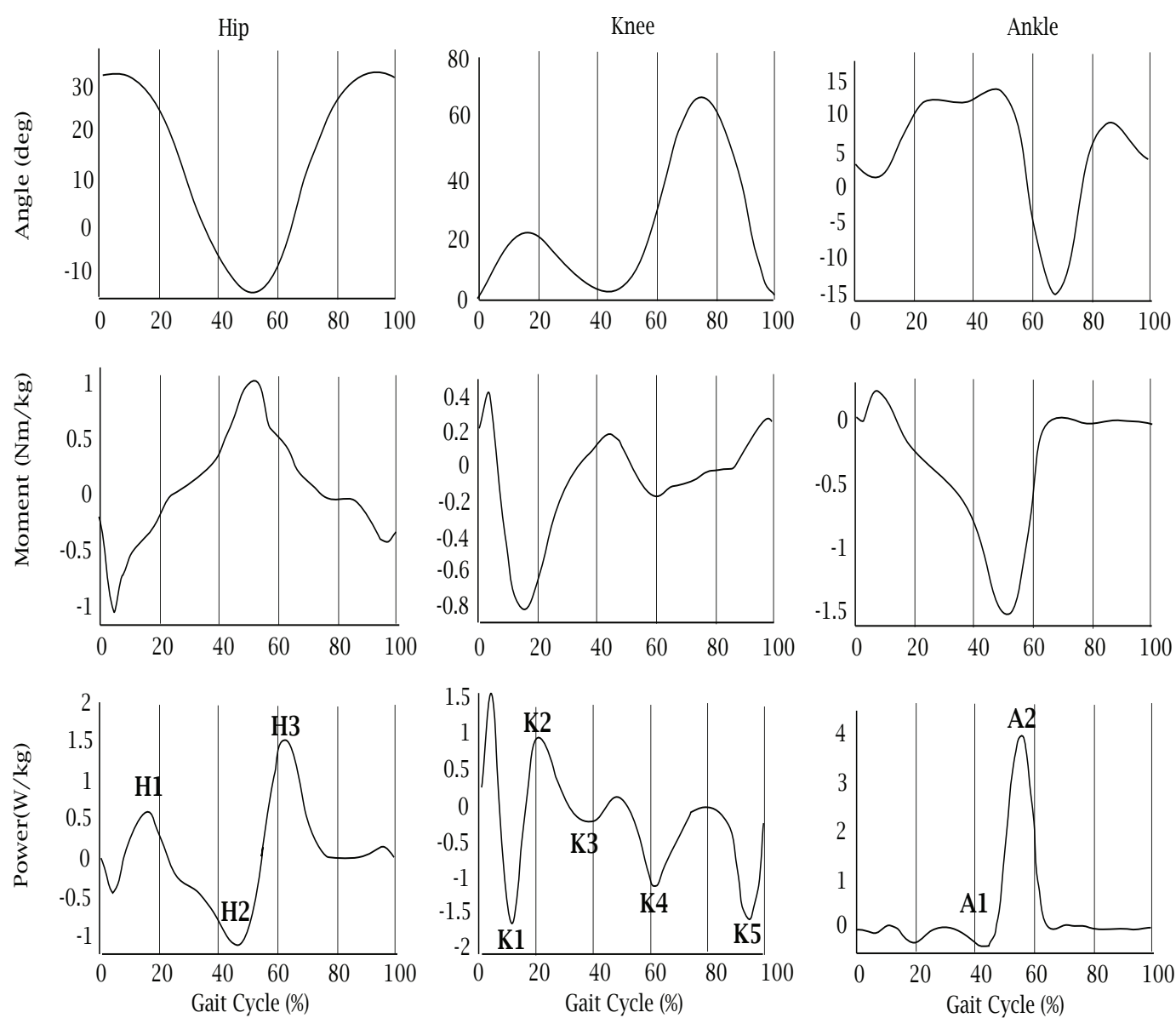

Figure 2.9: Angle, moment exerted and mechanical power of each joint in a gait cycle in the sagital plane [5]. 
Analyzing the moment curves, it is remarkable that in the swing phase moments are clearly lower than in stance in every joint. In hip and knee, during swing phase, the forces do not reach one quarter of the force required in stance. In the ankle the force needed during swing is negligible.

- Hip - The high extension moment at heel strike is needed to control the impact of the body with the ground and helps to maintain the trunk vertical. A flexion moment in mid stance is required to begin the change of direction of the leg. The moment before heel strike produces the flexion that prepares the body weight acceptance.

- Knee - In early stance, just after heel strike, an extension moment followed by one of flexion, accommodating the body collision with a soft loading response. During mid-stance a reduced moment is recurred to let the inverted pendulum behavior of the body. Therefore at the end of stance and at the beginning of swing, a moment is needed to lift the shank for foot clearance. During mid-swing this torque is held to support the mass of the shank and the foot and to control the inertia, and in the final phase of mid-swing this torque is released while the leg moves taking advantage of gravity. In the end of swing a moment is needed to slow the shank before heel strike.

- Ankle - The dorsiflexion moment injected just after heel strike helps to slow down the body motion. After the loading response, the plantar flexion moment allows the body to roll over the foot. At the terminal stance and preswing phases a sharp increase in the moment is observed and a large peak is observed in ankle power, this corresponds with the toe-off impulse that redirects the center of mass of the body. Immediately after, the foot is sent to a neutral position to provide foot clearance with no power consumption during all the swing phase.

\subsection{A model of human gait}

To understand human walking, to quantify energy consumption in each gait phase and to extract the main features that allow us to build a low consumption gait for an 


\subsection{A model of human gait}

orthosis, a model is needed. Walking is a process with a highly nonlinear, coupled, and generally unstable dynamics. The dynamics also switch from the phase of stand to swing, therefore an hybrid model must be accomplished. The model will make simplifications based on assumptions that allow us to understand such a complex behavior. Traditionally, when models of the human gait are established, it is said that a hybrid model is used when two models are used, one for the swing phase and another for the support phase. To merge both models, boundary conditions are used, defining an impact model. Therefore, in this thesis, algorithms will be established based on hybrid models. In general, the gait will be divided in different phases to apply different algorithms in each one of them and in the boundary points of union of each phase the conditions for a smooth gait will be established.

The design of a robot usually comes inspired by elements that already exist in nature. The characteristics and strategies that humans use to reduce energy consumption, mentioned in the previous sections, provided the basis for the development of robots with a reduced power consumption compared to conventional developments. In turn, the development of energy-efficient bipedal walking robots has deepened knowledge of biomechanics of human walking.

This section presents and discusses models of biped-robot walkers with low energy consumption and the strategies that they utilize to achieve such an economic operation. The discussion will focus on these strategies and their potential application to active orthosis and also include an analysis of the limitations that nowadays technology in engineering encounters.

Reviewing examples of bipedal robotic walkers with low power consumption, it can be observed that the robots that achieve lower consumption, are not versatile enough. They are just designed to walk in a limit cycle with very low energy consumption. For example, they are not able to stop and walk again by themselves or to sit down and stand up. These robots exploit the passive dynamics of their limbs to move with none or very few power from actuators or including actuators but not directly being used for the propulsion of motion.

The robots with a more versatile performance tend to include rigid motors and gearboxes with a very high reduction ratio needed for the high joint torque required. Therefore they tend to cancel the intrinsic dynamics of the limbs of the robot, caus- 
ing a very high energy consumption. These methods will be analyzed in depth in the next chapter.

Accordingly, given that there are no ideal actuators, in this chapter the main characteristics required by the actuators to achieve a natural and efficient walking are established.

The gait cycle efficiency is determined by two critical points:

- The energy consumed by actuators that move the walking robot. Generally, the actuators are not able to absorb and dissipate energy.

- The mechanical energy lost during the gait cycle, more particularly, the energy lost on the impact of the foot with the ground at the end of each step.

The human muscle model will be the basis for the analisys of the actuator features. As was reviewed in the previous chapter, muscles accomplish stiffness variation thereby achieving versatility and low power consumption.

From the perspective of robotic engineering, the step from passive or quasi passive robots that achieve a reduced energy consumption towards more versatile robots, illustrates the necessity of joints or actuators with variable stiffness, a feature that human muscle-tendons units have. As a result of this controllable stiffness, it is possible to exploit the dynamics of the limbs and to harness the energy that is stored in passive elements.

After all, an active orthosis or exoskeleton is a robot coupled to the lower limbs of a human, that walks. Therefore, to explore strategies for reducing energy consumption in the design and control of a robotic orthosis it is necessary to review and analyze developments in walking robots with low energy expenditure.

The models proposed in this section will be the base to define the strategies to reduce energy consumption while walking with an active orthosis. In addition, these models will be the frame to quantify, at least from a theoretical point of view, the energy consumption in certain critical phases of gait.

\subsubsection{Kinematic model of walking}

The first model of walking analyzed in this thesis is the kinematic. The dissertation made in this section is based on the approach by Richard Baker [64]. The criteria 
in which this kinematic model is based is that while walking the following features must be achieved:

- Support of body weight.

- Clearance in swing.

- Adequate step length.

- Smooth transition.

- Energy conservation.

Analyzing the motion of the center of mass of the body, there is a clear similarity between the single support phase and the model of an inverted pendulum. The mass of the body pivots on the center of pressure of the foot. Figure 2.10 shows an inverted pendulum model in which the length of the pendulum $l$ corresponds to the leg length and the point mass $m$ corresponds to the body mass. A given amount of energy is needed to start the movement and then the mass keeps moving without requiring energy injection. The inverted pendulum is a mechanical model of a mechanism for moving an object forward without required energy. Because in a legged robot less than one third of the total mass is in the legs, moving the body during the single support phase following an inverted pendulum model is energetically efficient.

As a first approach, it is supposed that no torque is applied on the pivot point. The equation of motion of this inverted pendulum is:

$$
\ddot{\Theta}=\frac{g}{l} \sin \Theta
$$

Supposing the mass moving from left to right, it begins moving with a initial velocity and converting kinetic energy into gravitational potential energy. At the highest point it will reach maximum potential energy and lower speed. Then the speed will increase until the moment of ground impact in which all potential energy is being converted into kinetic energy.

Using this simple model for the single support phase, the body is supposed to pivot around the ankle joint, but the trunk must be upright. Therefore, in this 


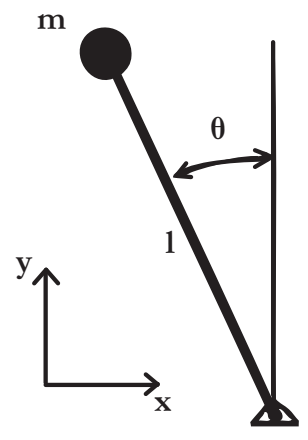

Figure 2.10: Model of inverted pendulum for single support.

preliminary model, the hip will follow a pattern opposite to the ankle pattern. This has been represented in Figure 2.11, the hip angle with this criterion almost follows the same pattern than the human gait hip movement. But the ankle pattern looks quite different.
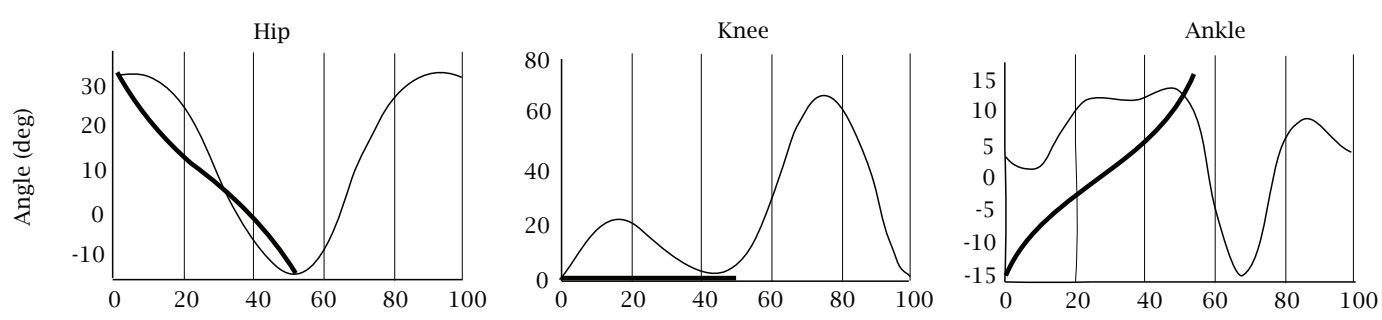

Figure 2.11: Joint angle patterns assuming the body pivoting on the ankle joint. The thin line represents the movement of each joint to follow the pattern of Clinical Gait Analysis (CGA). The thick line represents the movement of the joints, only in the support phase, assuming the body pivoting on the ankle joint following an inverted pendulum pattern and compensating the movement with the hip to maintain the trunk upright.

Being the motion pattern quite different, the energetics of the center of mass of the human gait almost looks identical to the inverted pendulum model.

The graphs in Figure 2.12 plot the horizontal and vertical component of the velocity of the COM at different speeds of walking. The black line follows the simple model of inverted pendulum during stance. Grey line represents the normal deviation of the movement of COM in human walking [6]. The horizontal com- 
ponent of the kinetic energy of the inverted pendulum matches almost perfectly the horizontal velocity of the COM of the body. The center of mass has its maximum velocity at the begining of the stance and reachs a minimum in the middle when the height is maximum and begins to accelerate while the COM lowers and potential energy is converted into kinetic energy.

However, in the vertical component the match is good in the mid stance phase but in the early and the end of the stance is far to match. Analyzing the model at different speeds, with slow walking and fast walking in Figure 2.12 it can be noted that horizontal predictions are equally good for the horizontal component but the discrepancies at the beginning and the end of the stance increase as speed increases.
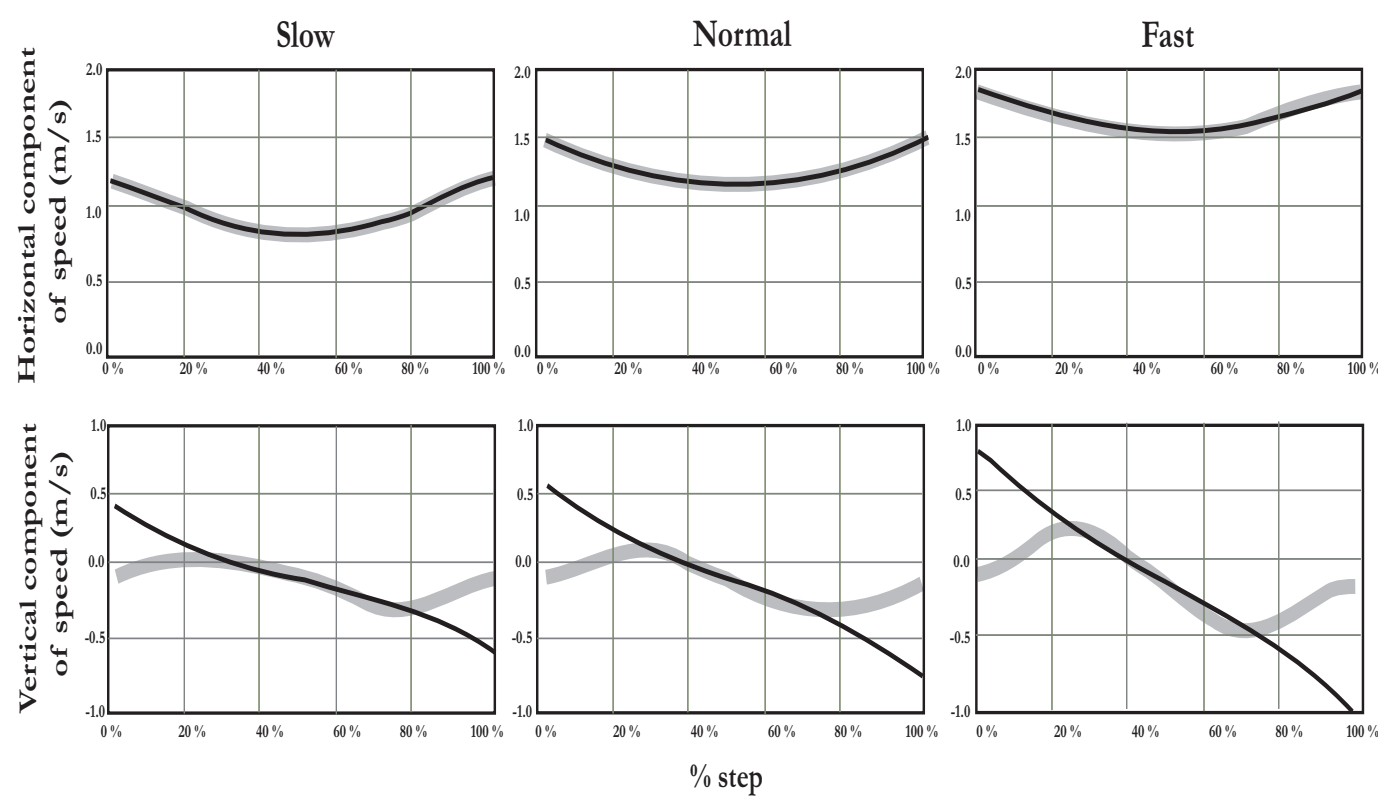

Figure 2.12: Horizontal and vertical components of the velocity of the COM at different walking speeds during the stand phase. Black line follows the simple model of inverted pendulum during stance. Grey line represents the normal deviation of the movement of COM in human walking. [6].

Most of the mass of the body is located at the Head, Arms and Trunk (HAT) and the inverted pendulum is an excellent mechanism for transporting that mass efficiently. The lower body has only about one sixth of the total mass. Then, it is not surprising that the way of walking fits around the mechanism that is more efficient for the langest posible amount of mass located at the COM. 
The simplest model of bipedal locomotion based solely on a sequence of inverted pendulums, is the compass gait. The features that define this simple model of walking are:

- The masses of legs and hip are point-masses.

- The gait consists of two stages:

A single support stage in which the system behaves as a double pendulum, following a pure ballistic motion.

An instantaneous transition stage, that means instantaneous double support. Therefore, stance and swing are both fifty percent of the gait.

- The collision of the swing leg is assumed to be inelastic, instantaneous and without sliding. In this collision, all the energy of the swing leg is transfered to the support leg to begin its swing in a ballistic motion.

In this model there is no knee movement and the ankles mirror exactly the motion of the hips.

The dynamics of this model is analyzed deeply in Chapter 3. Even as a very simple model of walking, it provides keys to achieve biped robot walking with very low power consumption, being inspired on how humans walk.

\subsubsection{Providing ground clearance}

Any model of bipedal walking must provide ground clearance to the swinging leg. In the compass gait model usually this ground clearance is provided by assuming telescopic legs. So the swinging leg shorters for ground clearance. However, human legs are not telescopic, so this assumption is not valid for exoskeleton applications.

Let's analyze how humans provide ground clearance and the behavior of the joints to achieve it (See Figure 2.13).

- The ankle must provide a dorsiflexion motion in order to avoid an exaggerated flexion of the knee and hip ( $\mathbf{C}$ in Figure 2.13). 
- Using the knee to prevent the toe to touch the ground in swing requires a lot of knee flexion but also requires earlier hip flexion ( $\mathbf{A}$ in Figure 2.13).

- Assuming the ankle is in neutral position, the minimum toe clearance occurs about half way through swing. To avoid the foot to touch the ground the knee must flex to at least $50^{\circ}$ by mid swing. (B in Figure 2.13)
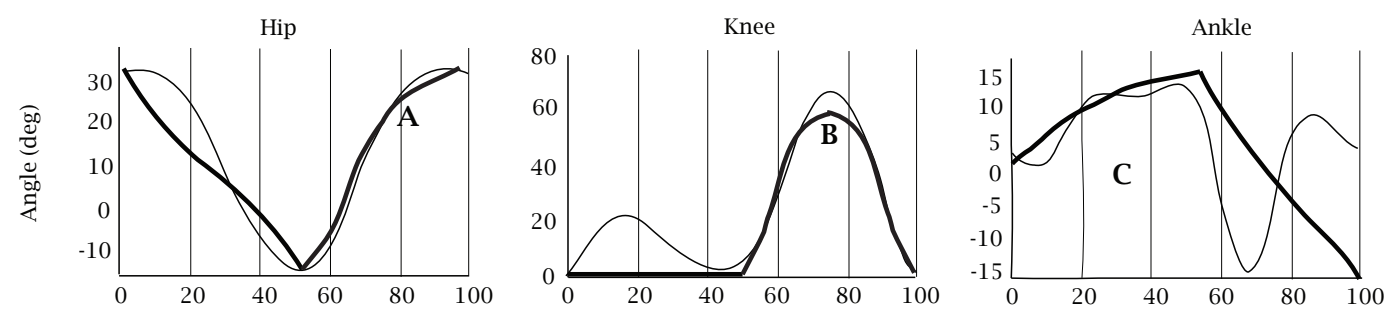

Figure 2.13: Joint angle patterns to provide ground clearance. The thin line represents the movement of each joint to follow the pattern of Clinical Gait Analysis (CGA). The thick line represents the movement of the joints assuming the body is pivoting on the ankle joint in the support phase, and providing ground clerance in the swing phase.

\subsubsection{Providing an adequate step length}

Step length is defined as the distance traveled between two successive foot strikes of the same foot. There is a linear relationship between step length and leg length which is dependent on the angle of the joints:

- Step length $(\mathbf{L})$ is primarily determined by the difference in hip flexionextension between the opposite foot and the contact foot. This is reflexed on the graph in the Figure 2.14

- A large step legth requires good knee extension at foot contact. (D in Figure 2.14)

- A large step legth requires some knee flexion before opposite toe off. ( $\mathbf{E}$ in Figure 2.14) 

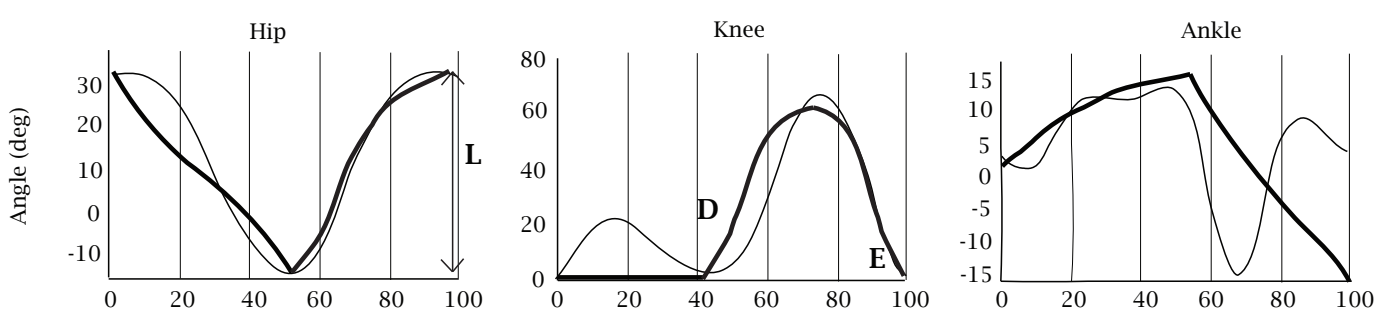

Figure 2.14: Joint angle patterns to provide an adequate step length. The thin line represents the movement of each joint to follow the pattern of Clinical Gait Analysis (CGA). The thick line represents the movement of the joints assuming the body pivoting on the ankle joint, in the support phase, and providing ground clerance in the swing phase. To provide an adequate step length the hip has to have simetrical flexionextension ( $\mathbf{L})$ between the opposite foot and the contact foot. In addition, the knee must have enought flexion and extension at the begining of the double support ( $\mathbf{D}$ and E) to provide a large step lenght.

\subsubsection{Support of the body weight}

Obviously in the process of walking, the vertical ground reaction forces (GRF) must be at least equal to the body weight to support the body and avoid falling down (Figure 2.15). However, the ground reaction force of an inverted pendulum is always less than gravity, due to the effect of the centrifugal force [65]. Then, an inverted pendulum is a very good way to move from an energetic point of view but it cannot support our body weight. To achieve a safe and steady support of the body weight, humans employ two mechanisms:

- The contribution of muscles: In fact the shape of the ground reaction force which is measured in human walking is mainly due to the contribution of the muscles, rather than performing an inverted pendulum [65]. This contribution changes the shape of the ground reaction force. The force that the muscle impress in the motion and the elastic reaction of the muscle tendon, produce a hump shape of the ground reaction force shown in the Figure 2.16 . As this figure shows, the average of the GRF of each leg is not enought to reach the body weight.

- A double support phase: If the human gait model is based on the inverted pendulum to allow the body moving forward with the contribution of the 


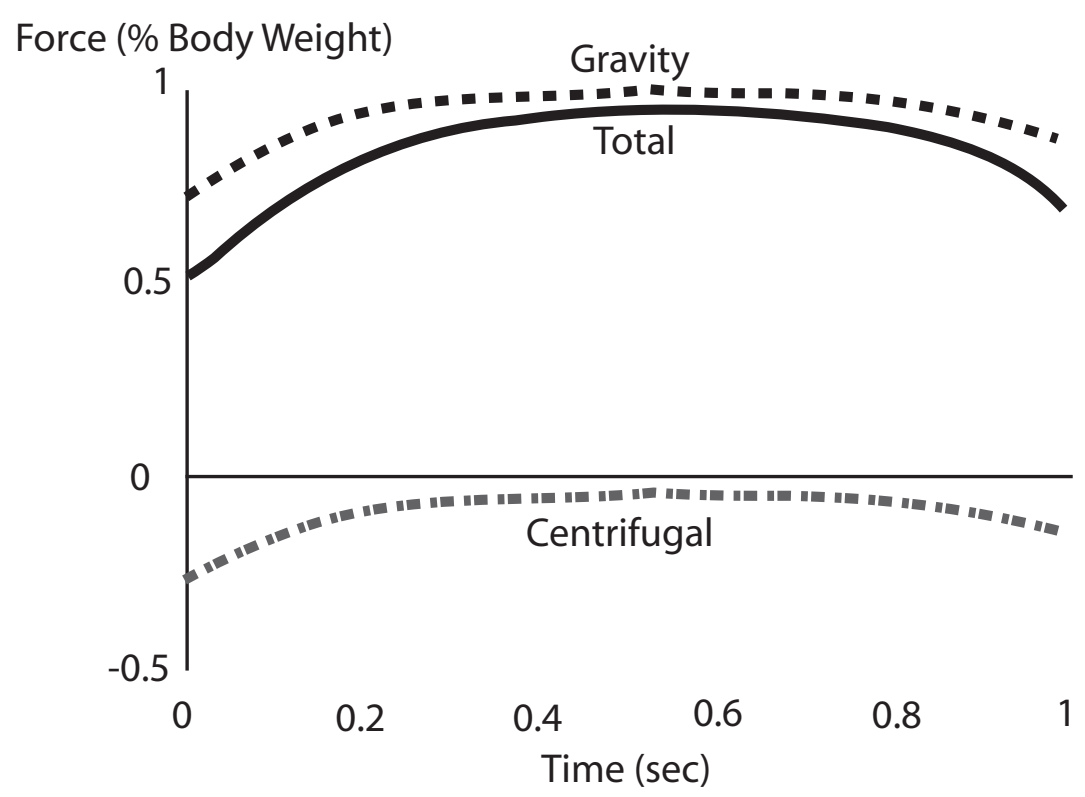

Figure 2.15: Ground reaction force of an inverted pendulum model. Due to the centrifugal force, the ground reaction force is less than the body weight.

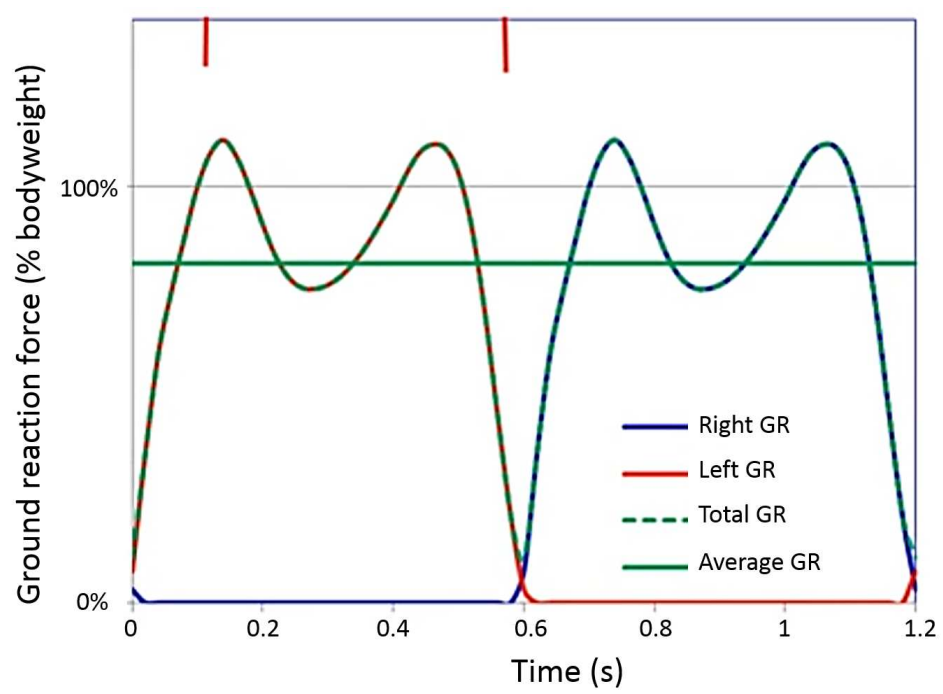

Figure 2.16: Ground reaction force of each leg. The average of the GRF of each leg is not enough to reach the body weight. 
leg muscle's, the GRF is not enought to support the body weigth. Then a period of double support is needed to ensure the body weight is supported. Moreover, as it is shown in Figure 2.17 a double support phase is necessary in order to support the body weight. In human walking double support is

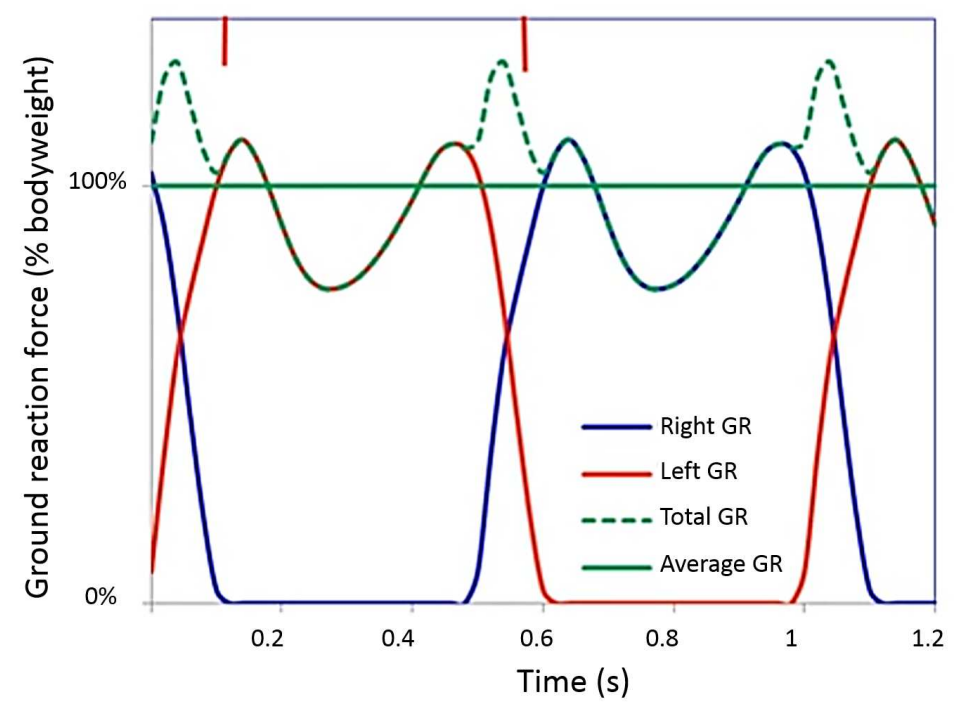

Figure 2.17: Ground reaction force of both legs together. The average GFR is enough to reach the body weight with a double support phase.

shown to be $10 \%$ of the gait cycle.

\subsubsection{Smooth phase transitions}

In human walking two major transitions between gait phases can be appreciated:

- Stance to swing at toe off.

- Swing to stance at heel strike.

As it will be seen in the next chapter, accomplishing a gait with smooth phase transitions improves stability and reduces energy consumption.

Achieving a smooth swing to stance transition is by far the most difficult action. In order to achieve a smooth heel strike, the velocity of the heel immediately prior to ground contact is virtually zero in the vertical direction and low in the horizontal direction. Heel speed at ground contact is less than $5 \%$ of maximum speed[66]. 
Figure 2.18 shows the horizontal heel velocity and how it is reduced to zero at heel strike to achieve a smooth ground contact. This zero velocity is imposed by the

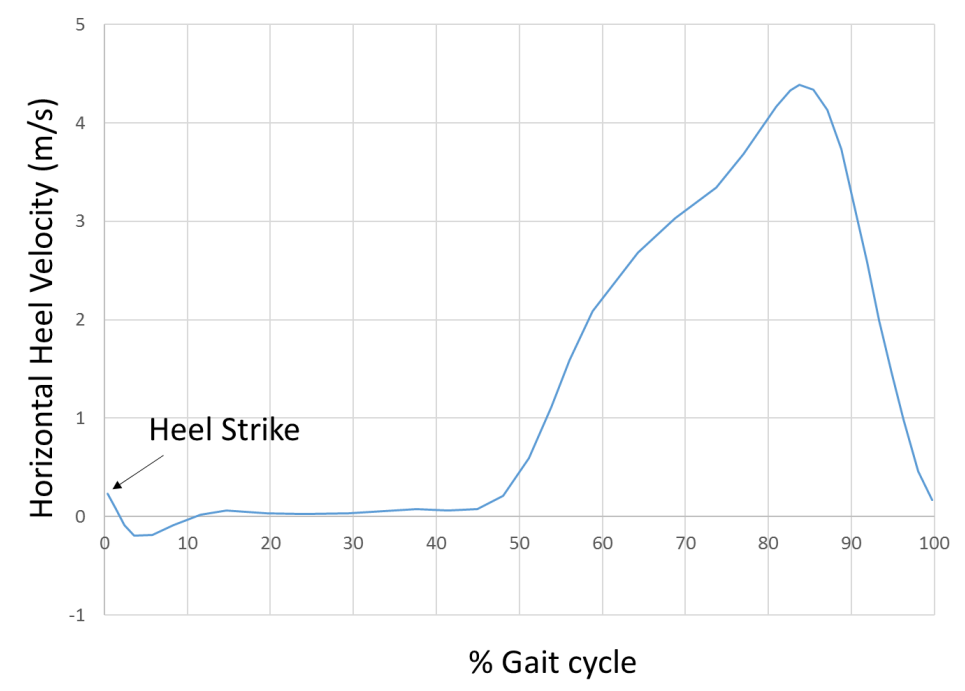

Figure 2.18: Horizontal heel velocity. At heel strike, it is reduced to zero to achieve a smooth ground contact.

motion control of knee and ankle as follows:

- The knee is flexing before heel strike, therefore reducing forward foot speed (see $\mathbf{F}$ in Figure 2.19 at the terminal swing and at the initial contact.)

- The plantar flexion of the ankle at heel strike contributes similarly. Therefore, ankle angle has to be approximately neutral and plantar flexing slightly prior to heel strike and this continues in early stance (see $\mathbf{G}$ in Figure 2.19 at the terminal swing and at the initial contact.)

Nevertheless, while the center of mass describes an arc of inverted pendulum during single support (see Figure 2.20) and then transfers instantaneously to the next single support, then a high impulse will be required for the transition between these two phases and this will produce a discontinuity in this transition, even having a smooth ground contact. To start with high velocity in the trailing leg we need dynamics transition from step to step. So, in order to smooth out discontinuities the force should not be applied instantaneously, the transition between inverted pendulum arcs has to be done gradually. 

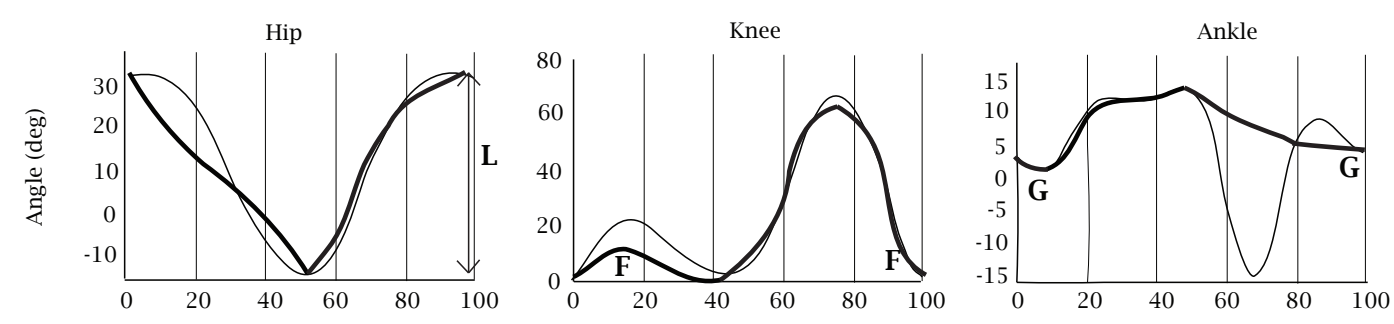

Figure 2.19: Joints angle pattern to provide an adequate foot contact. The thin line represents the movement of each joint to follow the pattern of Clinical Gait Analysis (CGA). The thick line represents the movement of the joints assuming the body pivoting on the ankle joint, in the support phase, and providing ground clerance in the swing phase. To provide a smooth swing to stance transition, knee $(\mathbf{F})$ and ankle $(\mathbf{G})$ modify their pattern at the terminal swing and at initial contact.

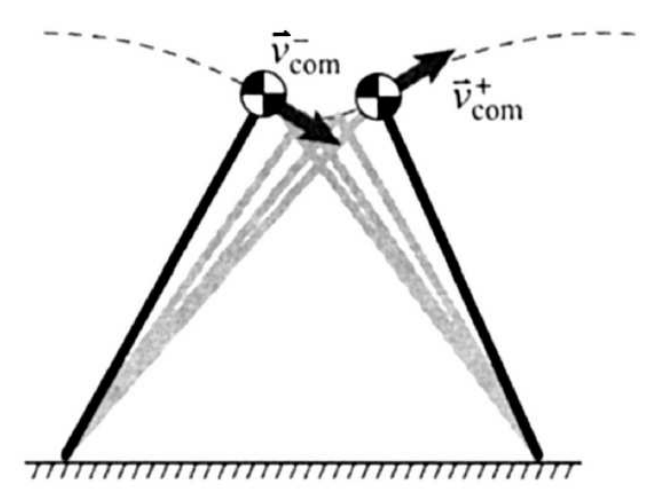

Figure 2.20: The COM velocity redirection is made by the flexion of the ankle of the trailing leg. 
At the end of the swing phase the center of mass is on the arc of the inverted pendulum and it has a downward component of velocity $\vec{v}_{c o m}^{-}$. By the time of ground contact, however, the horizontal velocity is nearly zero. Most of the deceleration of the COM on the vertical component of the velocity is achieved in the terminal swing before the heel strike, by the impulse of the trailing leg. This impulse is done by the plantar flexion in the ankle of the trailing leg at terminal stance(H in Figure 2.21). During double support both feet are in contact with the ground, the trailing leg rotates about the toe and the leading leg rotates about the heel. To provide even more heel rise, the knee flexion (I in Figure 2.21.) is increased while the ankle provides force by plantar flexion.
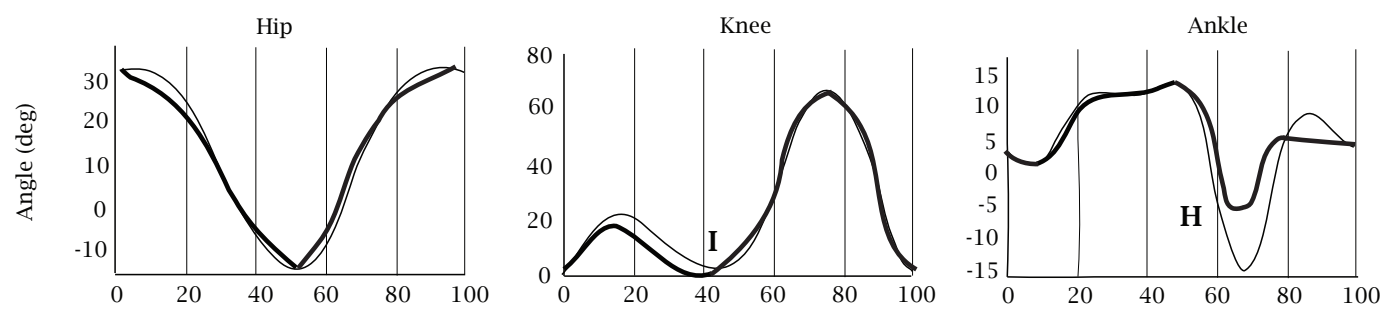

Figure 2.21: Joint angle patterns to provide plantar flexion at toe-off. The thin line represents the movement of each joint to follow the pattern of Clinical Gait Analysis (CGA). The thick line represents the movement of the joints assuming the body pivoting on the ankle joint, in the support phase, and providing ground clerance in the swing phase. The impulse of the ankle in plantar flexion $(\mathbf{H})$ provides a smooth transition at the leading heel strike, while the knee flexion (I) improves the kinematics for the impulse.

\subsection{Model of the human musculoskeletal system}

The musculoskeletal system provides support and produces movement for humans. In this section a shallow description of the human musculoskeletal system is presented with the aim of summarizing the major features that make human locomotion so efficient.

To have a global view of how muscles and tendons move a joint, a muscle model is presented, then an overview of muscle-tendon unit (MTU) is introduced and 
finally a brief but very illustrative idea of how the whole musculoskeletal system works is described.

The models that are presented in this section have been selected for their good perspective about how the muscle-tendon structure configures its intrinsic property of compliance and its capacity of modifying this compliance.

\subsubsection{Muscle model}

Two types of muscle models are typically used in biomechanics. The Hill model[67] is a mechanical muscle model built from a mechanical engineering perspective. It describes the mechanical input-output characteristics of muscles. The second type of muscle model, proposed by Huxley[68] is based on the composition and structure of the muscle. It was developed taking into account the molecular structure of the muscle and predicts the generated forces simulating the links between the actin and myosin molecules that compound the muscles. Huxley model provides a high-level precision but it requires complex calculation and greater computational requirements. This is the reason why we used Hill's model, which is intricate enough to understand the mechanisms of energy savings in the muscles as a source of movement. On the other hand, the Hill model fails to provide any data on the internal details of the muscle, but it does not affect the scope of this study.

Hill's model is presented in Figure 2.22, it represents the mechanical response of human muscles. This model is constituted by one serial nonlinear elastic element $(S E)$ depicting the intrinsic rigidity of myofilaments, another nonlinear elastic element $(P E)$ but in parallel representing the binding muscle tissues and a contractile element $(C E)$ depicting active fascicles in muscles. The parallel element is responsible for the muscle passive behavior when it is stretched, even when the contractile element is not activated.

Muscles have also a viscous damper $\left(B_{C E}\right)$ as velocity response to a stimulus that is represented in the contractile element $(C E)$.

\subsubsection{Model of muscle-tendon unit (MTU)}

Each muscle-tendon unit (MTU) includes a muscle and a tendon joined in series, at a pennation angle $\alpha$ (see Figure 2.23) where $l^{M T}, l^{M}$ and $l^{T}$ are the length of the 


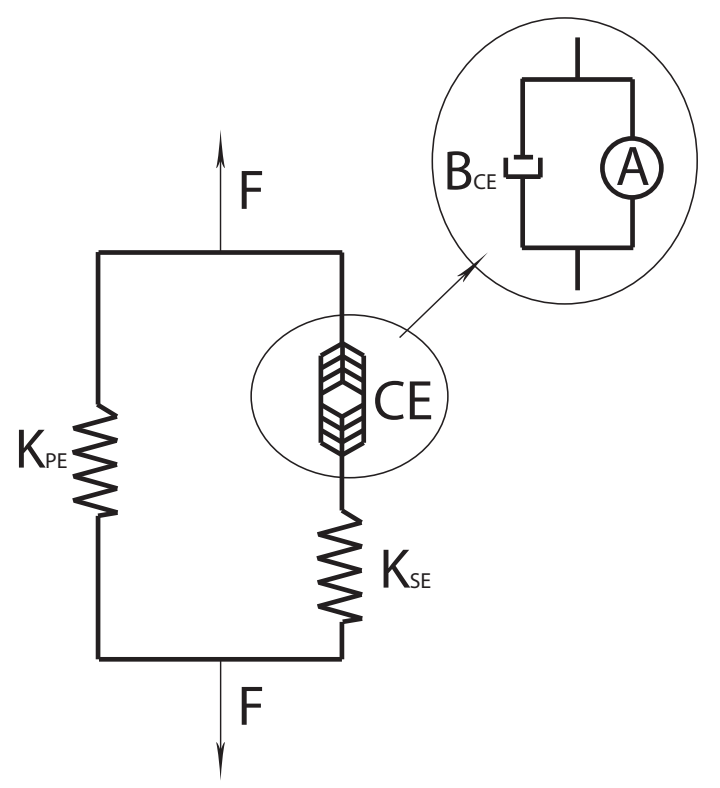

Figure 2.22: Simple model of human muscle (Hill 1993)[7].

muscle-tendon system, the muscle and the tendon respectively. Tendons represent a nonlinear elastic element in series.

From this model a comparison between motor and muscle can clarify their behavior and show a new perspective to design actuators based on muscle features. Motors can produce forces very rapidly while muscles are springy and viscous which makes them produce force slowly. Furthermore, this springy and viscous behavior endows with an intrinsic elasticity that helps to absorb unexpected impacts. Tension in the muscle depends on muscle length and joint angle, while in motors the torque does not depend on joint angle.

The muscle-tendon unit can only exert contractile force. So, for a birectional motion of the joint, they must act as an antagonistic pair of muscles. Figure 2.24 shows how agonist and antagonist muscles provoke flexion and extension in the elbow joint. This provides a critical feature of human joints that is the capacity to change the impedance of the joint mechanical response. If in the antagonistic pair of MTUs both are left without force then the joint can move freely. If both pairs of MTUs are left strained then the joint will be very rigid. Varying the force of the antagonistic pairs, any intermediate stiffness can be reached, allowing handling tasks from thick to very delicate. This allows the joint to have a wide range of 


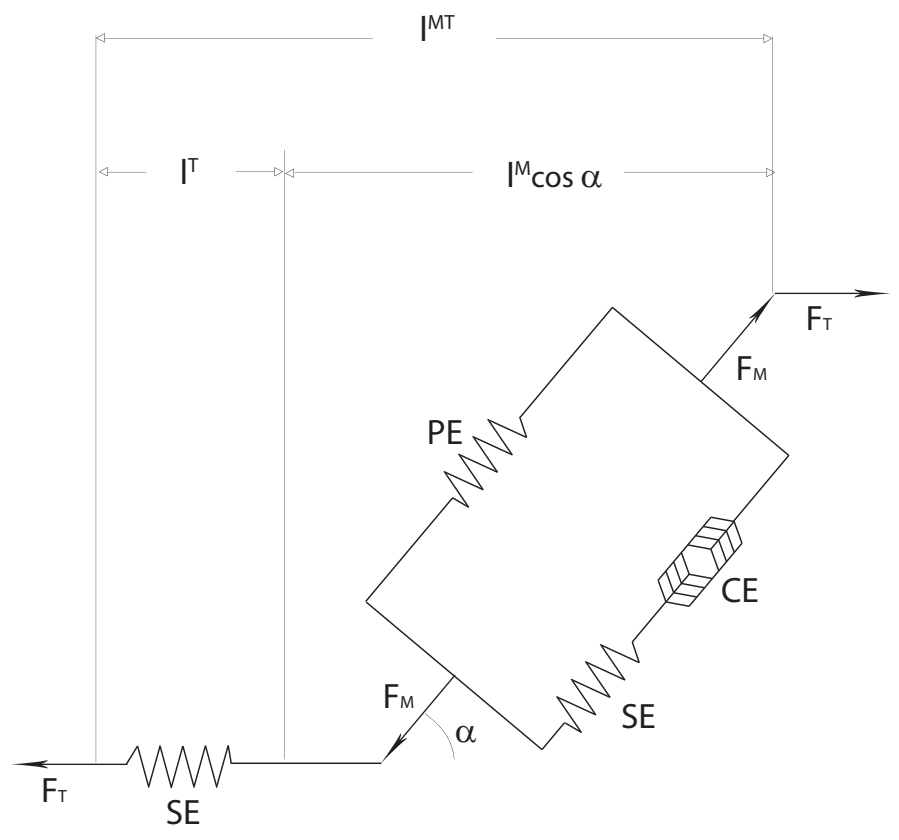

Figure 2.23: Simplified model of muscle-tendon unit[7].

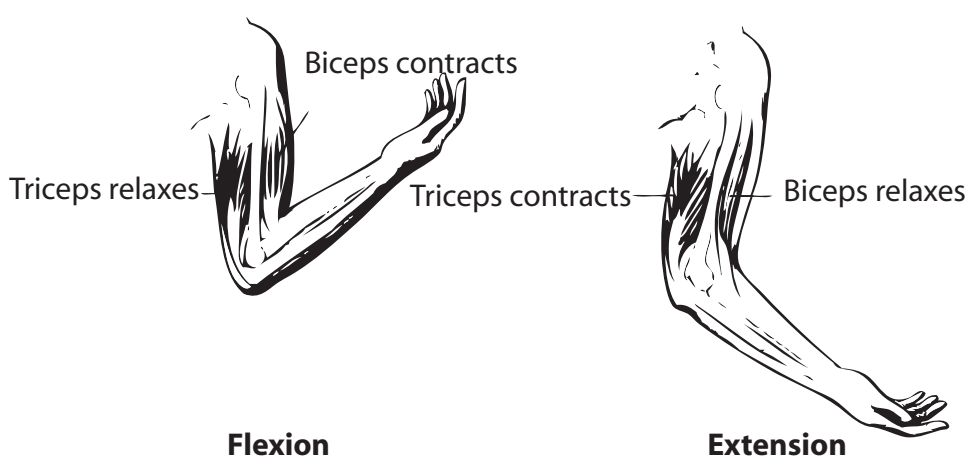

Figure 2.24: Flexion and extension in the elbow. 
variable stiffness, if both muscles act at the same time an isometric action (without motion) takes place and the joint achieves high stiffness, if only one muscle is activated the joint will be moved to one side, if both muscles are deactivated the joint will show a free motion only with the stiffness of the tissues that compose the muscles.

Therefore, from an engineering perspective, a muscle-tendon unit represents an actuator with variable stiffness and capable of storing and releasing energy in compliant elements.

In the motion of a complex joint of the human body, not only a pair of muscles with their associated tendons is activated; a group of muscle-tendon units are involved and act redundantly and synergistically.

Some muscle-tendon units are biarticular, crossing two or more joints, and helping to improve the energy consumption of each individual MTU when the synergist motion of the joints is exploited. For example, the gastrocnemius runs from the heel to the thigh just above the knee, this biarticular MTU has a major contribution in the toe off impulse needed at the end of stance.

\subsection{Conclusions}

The purpose of this chapter was the understanding of the human gait from an energy point of view. Therefore, in this chapter, the movement of each joint in the sagittal plane of the human gait on level ground has been analyzed. The kinematics and the kinetics of the leg joints have been examined deeply, and a kinematic model, based on an inverted pedulum, has been presented. This model has been successfuly completed to achieve clearance at the swing, an adequate step length and to provide smooth transitions between each step.

Also, in this chapter, a model of muscle-tendon units is presented showing how they modify the impedance of the joint providing high dynamic capacity. With all this understanding it is posible to compare other robotic systems.

In the next chapter, a comparision bewteen eficient and versatile robots is made. The advantages and disadvantages of each of these types of robots are presented and it is concluded that in order to exploit the benefits of both robots it is necessary 


\section{HUMAN GAIT}

to have joints capable of varying their rigidity and also to have algorithms capable of exploiting this ability to achieve a low energy consumption. 
Whether we are based on carbon or on silicon makes no fundamental difference; we should each be treated with appropriate respect.

Arthur C. Clarke

\subsection{Introduction}

In the previous chapter, an analysis of the features that make the human body an extremely efficient machine for walking at different speeds and types of terrain has been presented. In this chapter, we move to the robot side to study the evolution of bipedal robots from the point of view of energy consumption and the different strategies that have been adopted. Since an active orthosis is a robotic device, the strategies analyzed here will be the basis for the development of motion algorithms to be implemented in the ATLAS exoskeleton. In this chapter new approaches, that go beyond the state of the art, are proposed. In turn, comparing those strategies with the biological strategies presented in the previous chapter, their convergece is shown.

\subsection{Economy vs. versatility, a trade-off}

Classical biped robots, that perform a wide variety of movements such as walking or climbing up and down stairs or even running, base their motion control system in 
following predefined joint trajectories, monitoring some stability criterion like Zero Moment Point (ZMP)[69]. In this scheme, actuators are continuously in operation during the whole gait cycle. This characteristic involves the disadvantage of high energy consumption. A famous biped robot like ASIMO by Honda[70], shows an specific cost of transport $\left(C O T_{\text {Asimo }}=3.2[\overline{71}]\right)$ approximately thirty times greater than a human $\left(C O T_{\text {Human }}=0.3[72]\right)($ See Figure 2.1).

However, more than two decades ago McMahon [73] introduced the concept of ballistic walking, where a mathematical model of the swing phase is presented. In this model, muscles act only to prepare the initial position and velocity of the limbs at the begining of the swing phase. Then the leg swings only by the action of gravity and the limb inertia. This model not only fits with the speeds measured in the human gait but also provide an approximation to a swing with high efficiency. Following the same paradigm, McGeer[74] demonstrated that a biped robot can walk down a ramp only with an initial impulse. Potential energy is converted in kinetic energy, while the excess of energy is dissipated with the ground impact.

In such a model the force of gravity compensates for the loss of energy that occurs in the impact with the ground, and a limit cycle is created [71]. For a robot walking down a ramp with no control input, a limit cycle exists if the kinetic energy dissipated at ground impacts is recovered during the single-support phase by transfering the potential energy of the robot. Therefore the initial condition must be selected with a comprehensive precision. The term passive-dynamic walking refers directly to this natural mode of locomotion. Passive because no external energy is applied to move the robot and dynamic in contrast with the static stability in which the projection of the center of mass of the robot is always inside the support region[71].

These early passive bipeds have a constant step length that depends on the slope of the ramp, but based on the principles of these studies, researchers have built efficient robots that walk on flat surfaces or uphill[75]. These robots exploit the passive dynamics of the leg but use actuators to compensate the term of the potential energy of the downhill ramp.

Whether descending a ramp or walking on level ground, active feedback control can be applied to inject energy into the gait during the single support phase 
compensating for the loss of energy in the ground impact. The biped robots that explode these techniques are called actuated or controlled passive walkers.

In contrast,while conventional biped robots use one acuator per joint, the passive dynamic walkers do not have actuators in most of their joints, acting underactuated.

To walk on level ground, based on this limit-cycle theory, robots are given only the required amount of energy to keep them within the limit cycle. The bipeds built in the University of Technology of Delft (see Figure 3.1][76] base their low energy consumption on the performance of the ankle giving impulse in the phase of pushoff. In a first model they use a spring to store the energy in the back leg and release it when it strikes the front. This model was not complete because, to keep the biped within the limit cycle, the energy had to be released before the impact of the front leg to help reduce its impact energy. So they did in their second model, ensuring that the foot landed correctly.

Another biped based on passive dynamic was designed and built at Cornell University (see Figure 3.1] [77]. The main features that made the design simpler, more efficient, and easier to control are:

- Only one degree of freedom. Both legs and torso are joined by a single passive degree of freedom. In turn each leg has a union with its opposite arm, producing a brace identical to the human.

- Knee joint lock. The knee joint includes a hiperextension limit and a locking mechanism, that engages when the knee reaches full extension at the end of the swing phase and keeps locked with full extension throughout the support phase. When the locked mechanism is off the knee rotates freely.

- Ankle design. Like the design of Delph University, the ankle extension provides a push off on the back leg just before ground contact of the front leg. A small motor helps store energy in a spring in the swing phase that is released at the right time to provide the boost in the pushoff. This motor is the one that provides the small amount of energy required to keep the biped inside the limit cycle. 


\section{BIPED ROBOT STRATEGIES TO REDUCE ENERGY CONSUMPTION}

- Foot design. In every passive dynamic robot, the feet have a curved design to roll in the stance phase and to provide the impulse in the right direction. Each foot has different curvature on the outside and the inside so that the bipedal oscillates from side to side as it rolls forward, providing higher ground clerance in the swing phase.
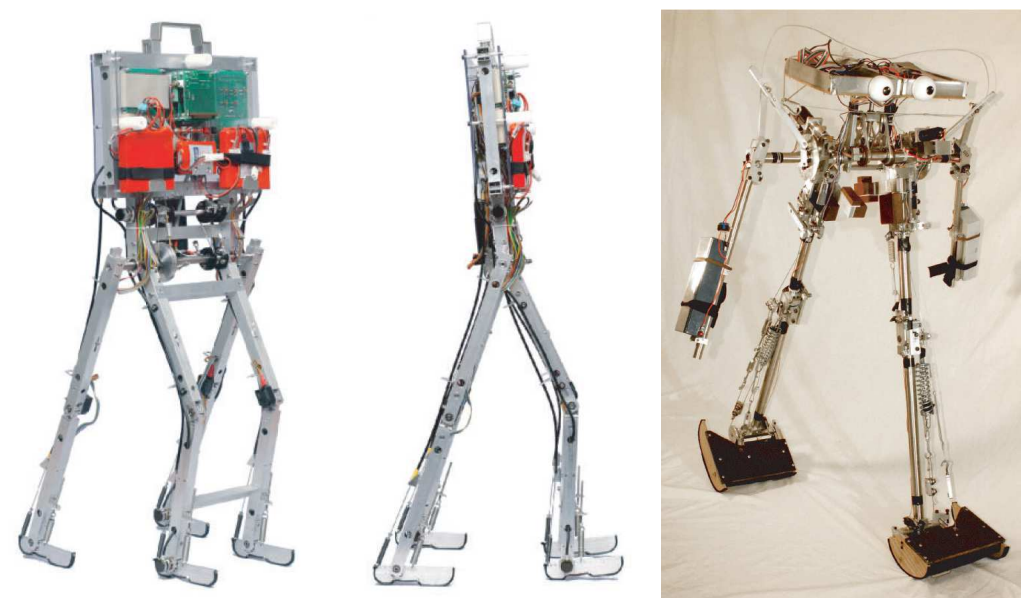

Figure 3.1: Passived biped robots. (a) Delph University and (b) Cornell University

The strategies that these bipeds follow to achieve a stable gait with a minimum consumption of energy provide a good guide for the development of efficient prostheses and orthoses.

\subsection{High energy consumption of stiff actuators}

Passive walkers are basically underactuated robots that exploit inherent dynamics. Conversely, the high-gain feedback that is usual in classical fully actuated robots, cancel the inherent dynamics of the robot. Let's analyze this other case indetail.

Actual robots and active orthoses use actuators with a high reduction ratio to provide the large torques require for locomotion. A simplified dynamic model of an actuator based on a direct-current (DC) motor an a gearbox (see diagram in Figure 3.2), which relates the input voltage $v$ applied and the output torque $\tau$ delivered by the motor and gearbox, is given by the equation: 


$$
J_{m} \ddot{q}+f_{m} \dot{q}+\frac{K_{a} K_{b}}{R_{a}} \dot{q}+\frac{\tau}{r^{2}}=\frac{K_{a}}{r R_{a}} v
$$

where:

- $J_{m}$ is the rotor inertia

- $K_{a}$ is the motor-torque constant

- $R_{a}$ is the armature resistance

- $K_{b}$ is the back electromotive force

- $f_{m}$ is the rotor friction coefficient

- $\tau$ is the resultant torque after the set of gears

- $q$ is the angular position of the load axis

- $r$ is the gear reduction ratio

- $v$ is the voltage applied

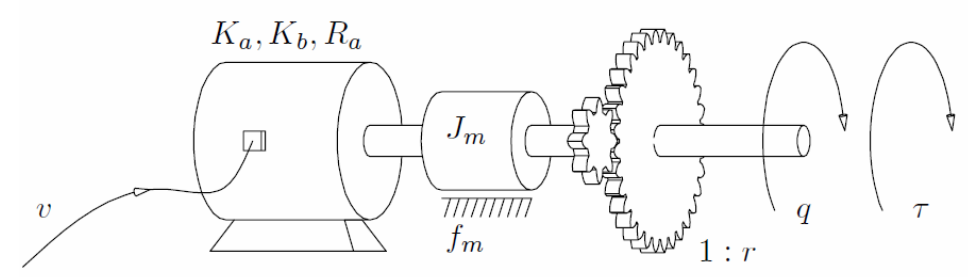

Figure 3.2: Model diagram of a DC motor.

Writing the equation for the $\mathrm{n}$ joints that are driven by the actuator:

$$
J \ddot{q}+B \dot{q}+R \tau=K v
$$

where

$$
J=\operatorname{diag}\left\{J_{m_{i}}\right\}
$$




$$
\begin{gathered}
B=\operatorname{diag}\left\{f_{m_{i}}+\left(\frac{K_{a} K_{b}}{R_{a}}\right)_{i}\right\} \\
R=\operatorname{diag}\left\{\frac{1}{r_{i}^{2}}\right\} \\
K=\operatorname{diag}\left\{\left(\frac{K_{a}}{R_{a}}\right)_{i} \frac{1}{r_{i}}\right\}
\end{gathered}
$$

for each actuator $(i=1, \ldots, n)$.

Therefore, substituting $\tau$ in the dynamic equation of the biped robot (3.2), the dynamic model of the biped that included the actuator at the joint is obtained:

$$
(R M(q)+J) \ddot{q}+B \dot{q}+R C(q, \dot{q}) \dot{q}+R g(q)+R f(\dot{q})=K v
$$

This equation considers the dynamics of the system with voltage $v$ as an input, position $q$ and velocity $\dot{q}$ of the joints. If the gear ratio of the actuator is large $\left(r_{i} \gg 1\right)$, then $R \approx 0$. For example, if $r_{i}=100$ then $R \simeq 10^{-5}$

The above equation (3.7),is approximated by

$$
J \ddot{q}+B \dot{q} \approx K v
$$

This equation indicates that, if the reduction ratio of the actuator is near to one hundred, which is a usual gear ratio in legged robots, the associated dynamics of the robot is canceled, and the dynamics of the actuators dominate the entire system.

It was shown in the previous chapter that to reduce the energy consumption of a robot it is necessary to exploit the intrinsic dynamics of the robot. However, if actuators with a large reduction ratio are used for joint motion, the natural dynamics of the limbs cannot be exploited. In other words, the conventional gear-motor approach for joint motion has to be modified for an efficient gait.

In addition to the underactuation joints approach to reduce energy consumption, another key approach to reduce energy consumption can be the use of compliant actuators.

In the next section, the state of the art of variable stiffness actuators is presented and analyzed. These designs do not conform to the features needed to be used in exoskeletons, as it is concluded in chapter 6 , due to their characteristics of torque, speed or range of stiffness variation. 


\subsection{Variation of actuator compliance to reduce energy consumption}

By analyzing the drawbacks of traditional actuators, with motor and gearbox from an energy viewpoint, to be used in the field of rehabilitation and gait assistance, several research groups have developed new actuators [78]. These new mechatronic developments have as main characteristics to be safe, efficient, compliant and of variable stiffness. The compliant actuators differ from rigid actuators essentially in that they allow deviations of the equilibrium position according to the applied external force[79]. This accommodation or compliance can be used to allow exploiting the robot inherent dynamics.

It is possible to make a classification of this type of compliant actuators, according to its different characteristics as presented in [80].

\subsubsection{Series Elastic Actuator}

A very important contribution in this development was made by $\mathrm{J}$. Pratt at the Mashashuses Technological Institute (MIT) with Serial Elastic Actuator (SEA) [81]. This type of actuator, (see Figure 3.3 includes a set of springs in the transmission, the elastic constant of these springs cannot be modified; but thanks to these elastic elements the contact force is fedback into the control loop, (see Figure 3.4).

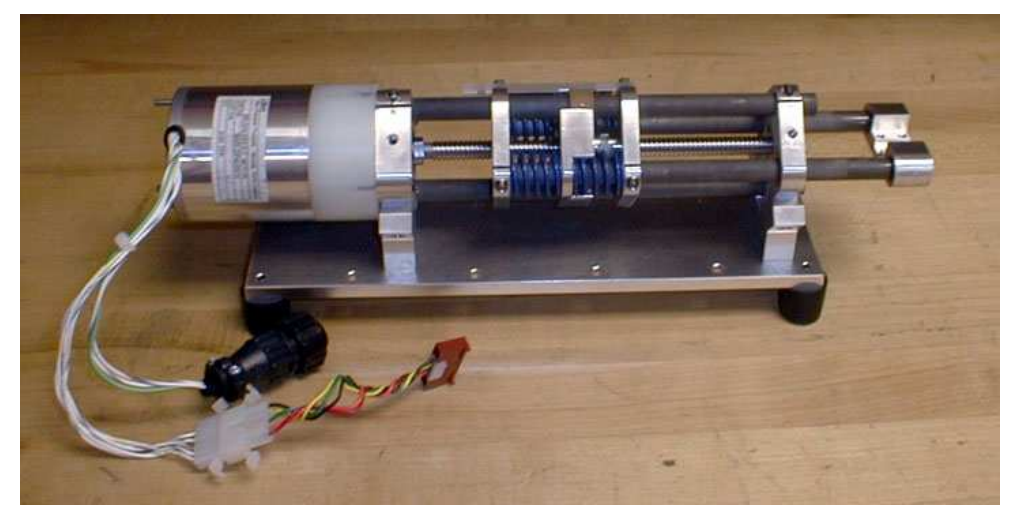

Figure 3.3: Series Elastic Actuator. Note the spring placed between the motor and the load. 


\section{BIPED ROBOT STRATEGIES TO REDUCE ENERGY CONSUMPTION}

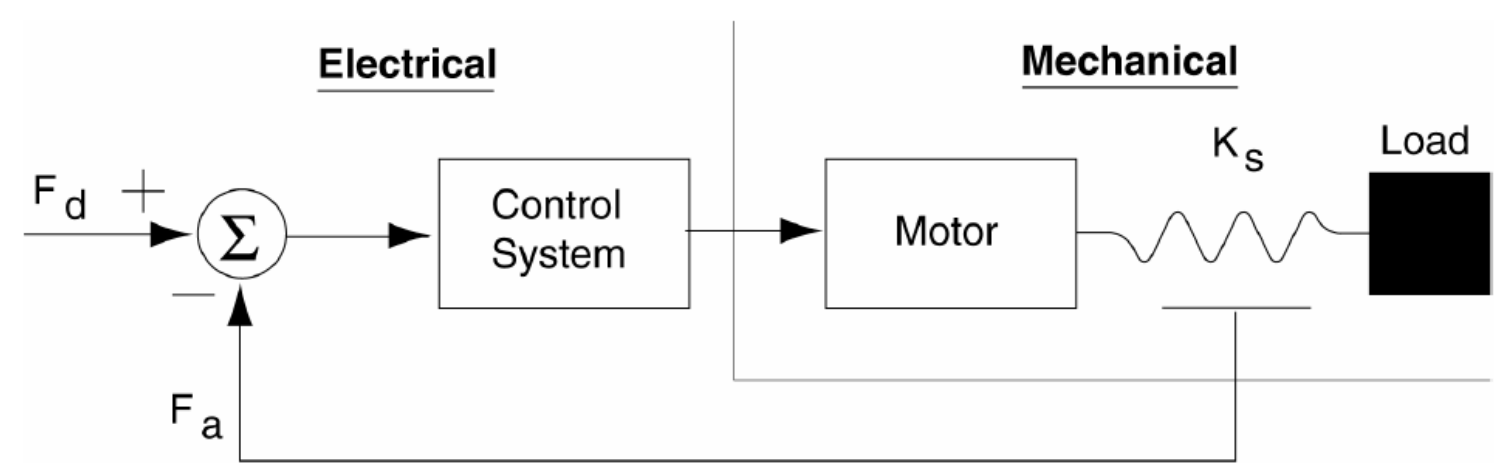

Figure 3.4: Control scheme of SEA. With compression of the spring, the force is measured and fedback.

Thus, force control becomes fast and efficient and the intrinsic compliance responds more safely to unforeseen interactions. This same concept including in rotatory actuators (RSEA) achieves the same characteristics of low impedance at the output of the actuator, high fidelity in position and strength. The quadruped of the ETH (Eidgenössische Technische Hochschule in its acronym in German) group of Zurich, uses high compliant series elastic actuation for the robotic leg ScarlETH[82]. Rehabilitation exoskeleton Lopes [40] and the most modern version of the IHMC byped [47] utilize this concept.

Another group of developments are based on modifying the stiffness of the actuator. According to the approach to stiffness variation, a classification is as follows:

\subsubsection{Antagonistic-Controlled Stiffness}

As we have seen in the Chapter 2, human muscles can only pull, not push. Thus, the joints of many animals and humans get to perform impedance variation by antagonistic pair of muscles. By analogy with human joints, various prototypes of varying stiffness actuators have been developed.

The Migliore's Biological Inspired joint[83] was developed at the Georgia Institute of Technology, (see Figure 3.5). From an antagonistic setup it achieves the stiffness variation by providing a quadratic variation of the force length relationship. 


\subsection{Variation of actuator compliance to reduce energy consumption}

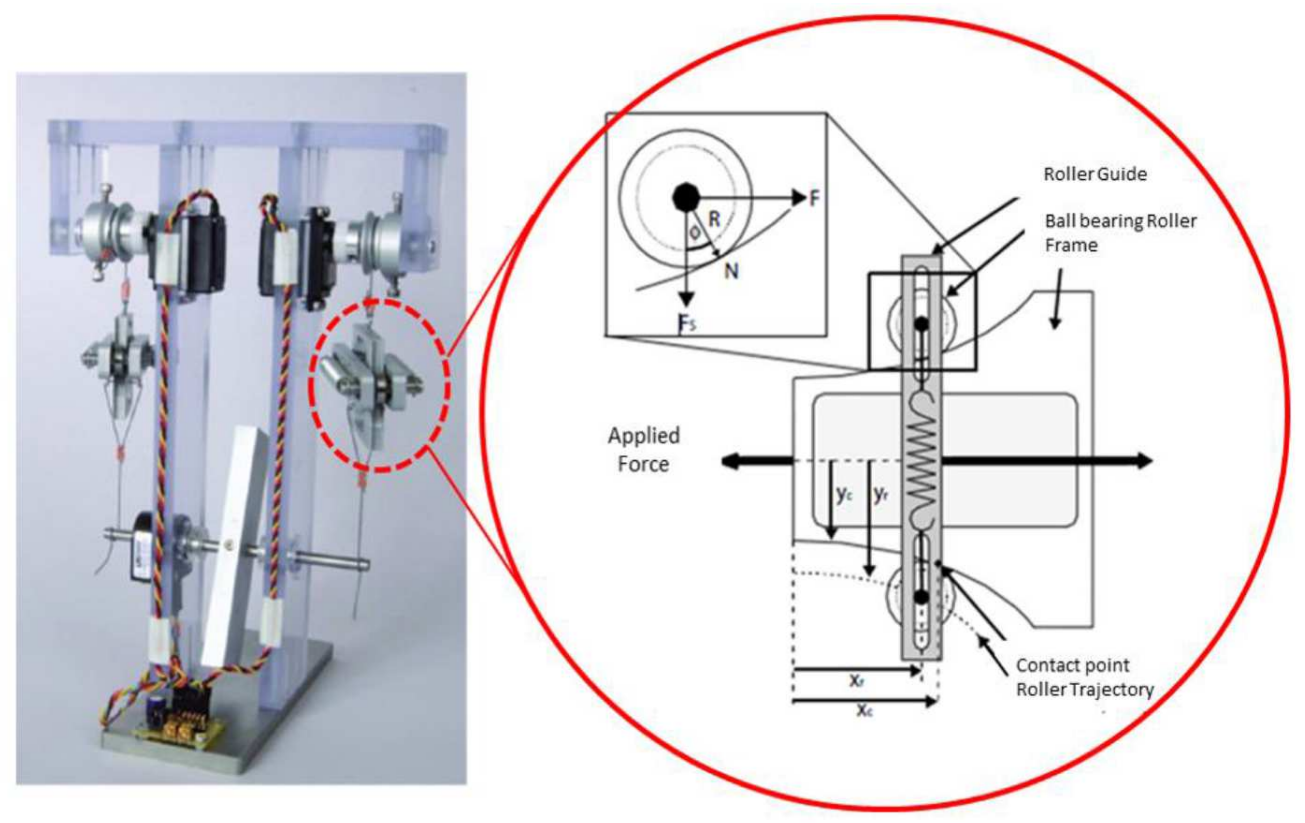

Figure 3.5: Migliore's joint with antagonistic setup from the Georgia Institute of Technology.

In the Lucy pneumatically actuated biped, (Vrije University Brussel[84]), a controller varies compliance for better performance of the robot in relation to energy consumption. The action of each joint is performed with two antagonistic actuators that are able to modify the compliance of the joint while they move it. From a known trajectory, it calculates the necessary torque based on the dynamics of the inverted pendulum to adjust the compliance of the actuator to the natural compliance of the desired trajectory.

Another ingenious design, also based on antagonistic configuration, is the Actuator with Mechanically Adjustable Series Compliance (AMASC) [85], which stands out because it only uses an actuator to vary the stiffness, is developed at Carnegie Mellon University. Its complex design (Figure 3.6) is also based on pulleys attached to elastic elements but the pulleys have a certain geometry that allow both linear and non-linear behavior of the elastic elements according to the task to be performed.

In the German Institute of Robotics and Mechatronics (DLR) they also developed the Quasi-Antagonistic Joint (QA-Joint) [86] based on an antagonistic 


\section{BIPED ROBOT STRATEGIES TO REDUCE ENERGY CONSUMPTION}

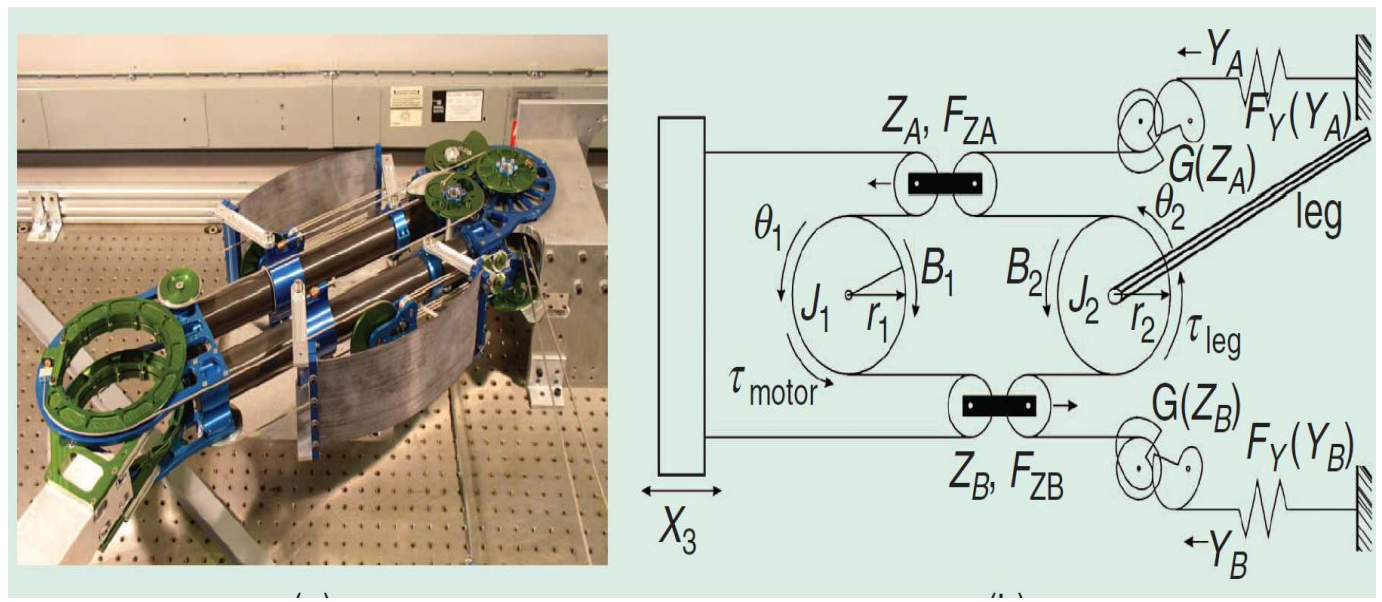

Figure 3.6: Actuator with Mechanically Adjustable Series Compliance (AMASC) from the Carnegie Mellon University.

configuration. In this design (see Figure 3.7) one engine takes care of the variation of stiffness while another one is in charge of modifying the angle of the joint. The variation of rigidity is achieved by two elastic elements, connected to pulleys that rest on a surface that moves the actuator modifying the rigidity.

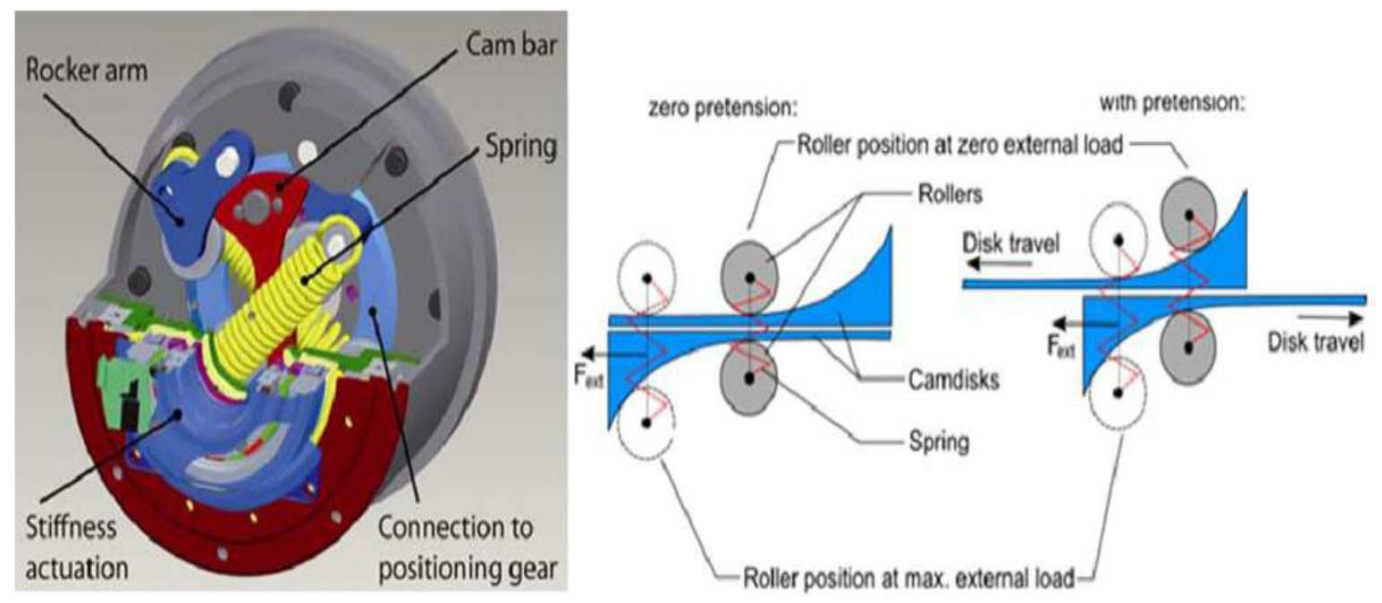

Figure 3.7: Quasi-Antagonistic Joint (QA-Joint) from the German Institute of Robotics and Mechatronics (DLR). 


\subsubsection{Variable Stiffness Actuators (VSA)}

At the University of Pisa, Tonneti et all [87] (see Figure 3.8) developed a device formed by three toothed pulleys joined by a belt tensioned by a pair of springs. One of them directly to the joint, the other two depending on the direction of actuation are able to compress more or less each spring producing the desired variation of stiffness.
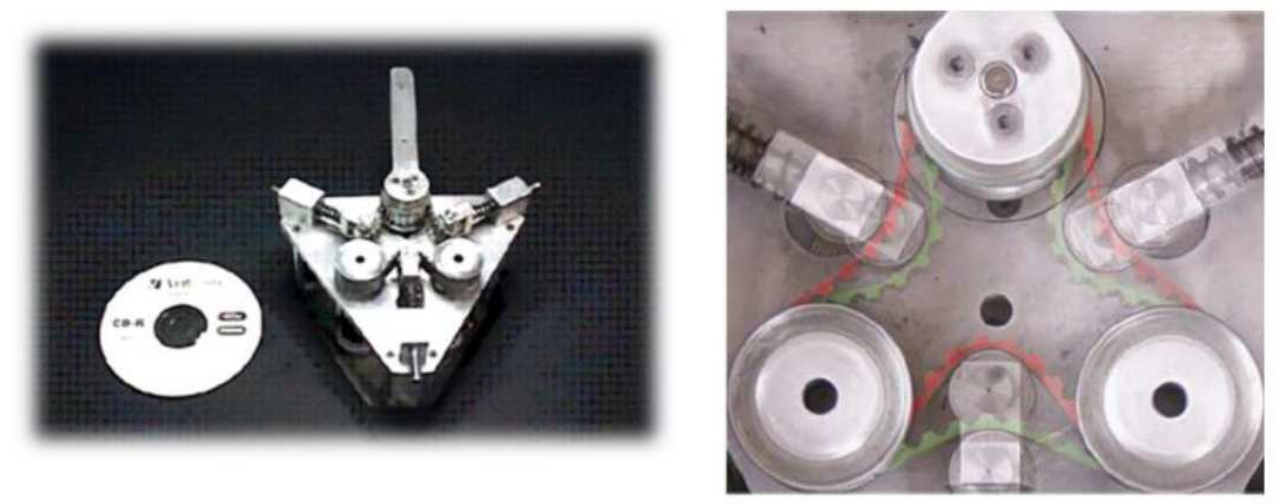

Figure 3.8: a) VSA Prototype b) Tensioning Mechanism.

\subsubsection{The preload adjustment of single spring}

By adjusting the point where the compliant elements are attached to the structure, stiffness adjustments can be made, independently from the joint position.

- MACCEPA

Mechanically Adjustable Compliance and Controllable Equilibrium Position Actuator is a design from Vrije Universiteit Brussel. In this development (Figure 3.9) an engine is responsible for varying the rigidity by prestressing a spring, while another is in charge of varying the angle of the joint.

- VS-Joint

The Variable Stiffness Actuator (VS-Joint) [1] from the DLR, Figure 3.10 contains two motors of different size, the high power motor changes the link 


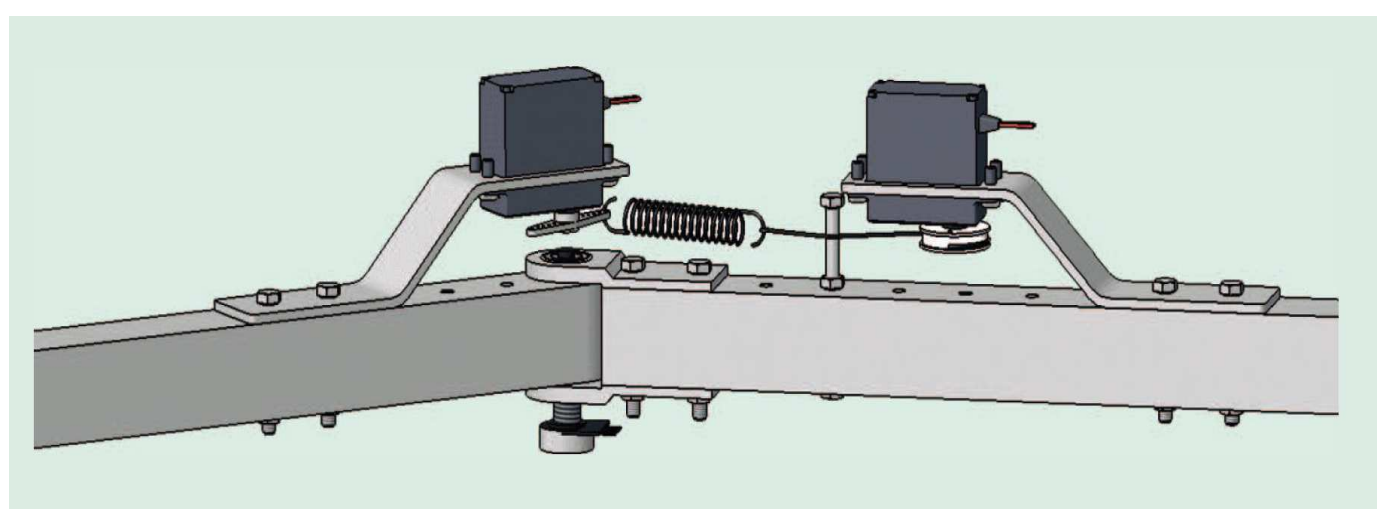

Figure 3.9: a) MACCEPA Basic Configuration. b) MACCEPA Prototype. c) MACCEPA 2.0 Schematic Drawing

position while the joint stiffness is adjusted by a much smaller and lighter motor.

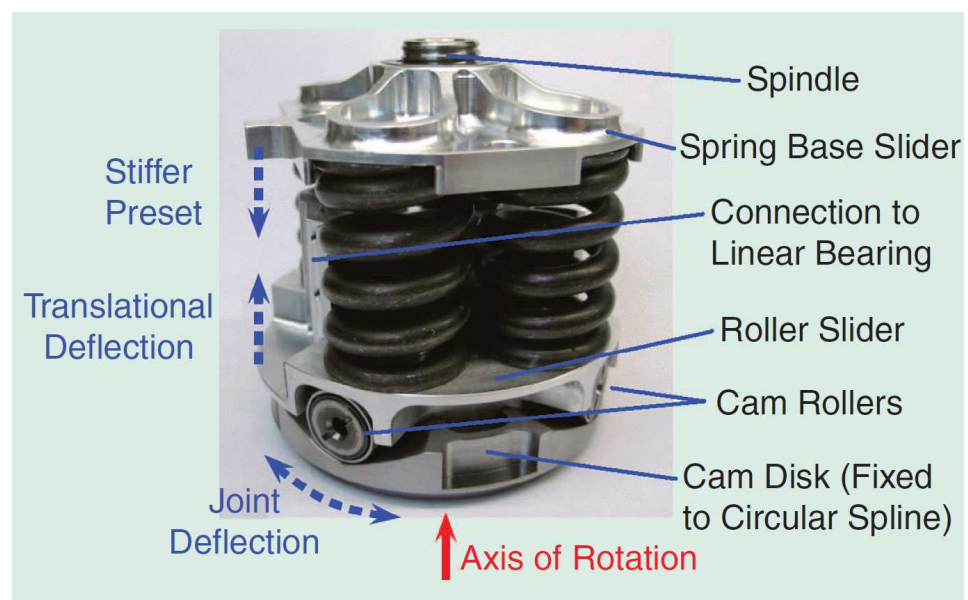

Figure 3.10: a) Mechanism of the VS-Joint. b) Working Principle of the VS-Joint.

\subsubsection{Changing transmission between load and spring}

These actuators regulate their stiffness by changing the transmission ratio in its mechanism. By means of adjusting the position of a pivot or the elastic elements along the structure. The main advantage of this group is that the adjustment doesn't 


\subsection{Variation of actuator compliance to reduce energy consumption}

act in the direction of elastic elements. Thus a small motor can be used to regulate the stiffness.

The Italian Institute of Technology has come up with several designs implementing this technique such as the AWAS and AWAS II[88]. The Actuator with Adjustable Stiffness (AwAS-I), see Figure 3.11, varies the stiffness thanks to two opposing springs coupled to a bar at a variable distance. The variation of the arm is caused by a motor that causes the stiffness to change. Another motor is responsible for getting the angle of the joint.

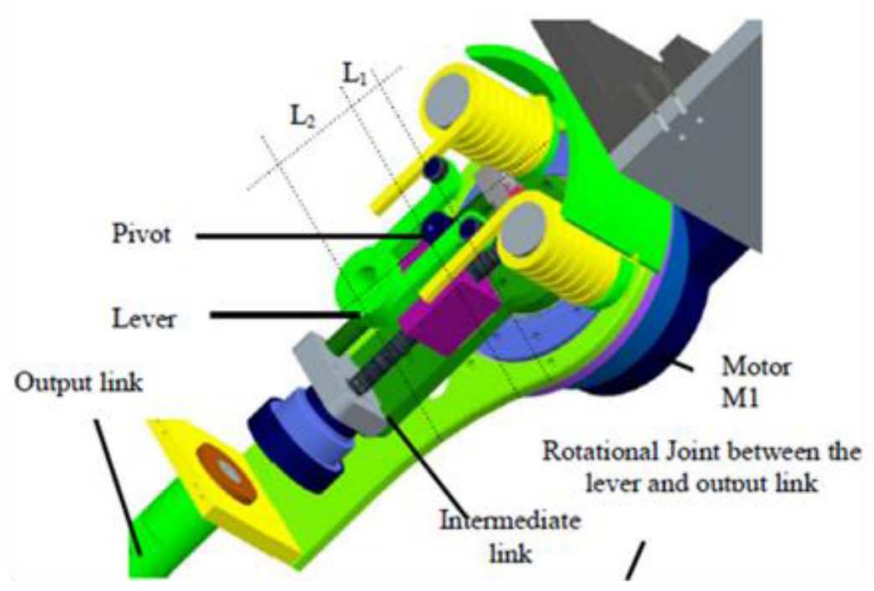

Figure 3.11: a) AwAS I Mechanical Principle and b) AwAS II Mechanical Principle.

Another development of stiffness variation of the Istituto Italiano di Tecnologia (IIT) is the CompAct-VSA[89], see Figure 3.12 a motor moves the transmission pivot inside a cam shaped lever arm, producing stiffness variation required. The required joint angle is given by another motor.

Table 3.1 shows the main characteristics of the mentioned actuators. It also shows the characteristics of the two actuators designed and developed for its implementation in the reduction of energy consumption.

In the paper [90] an in-depth analysis of variable stiffness actuators is made from a point of view of energy consumption. This paper concludes that energyefficient variable stiffness actuators are that in which the apparent output stiffness can be changed without the injection or extraction of energy to and from the internal elastic elements. Therefore, actuator designs that use a pretension mechanism to 


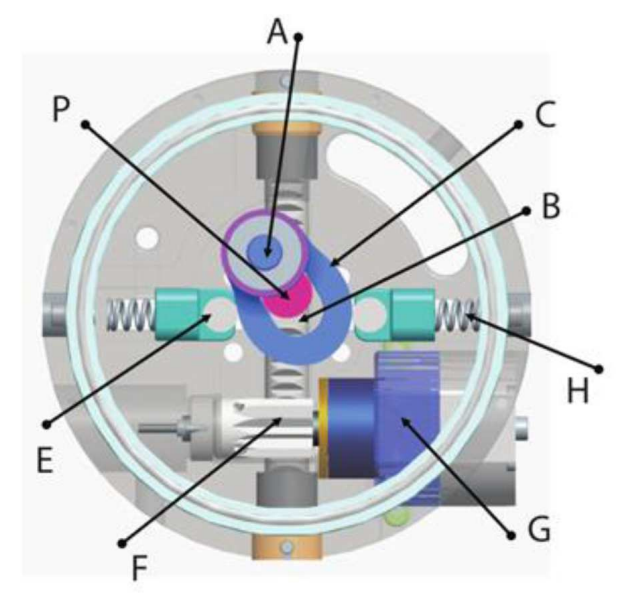

Figure 3.12: CompAct-VSA Mechanism.

the change of the apparent output stiffness are not energy efficient. That designs based on the antagonistic principle, e.g., like VSA II [87], are not energy efficient. Those designs that use pretension to change the apparent output stiffness does not achieve energy efficiency for the same reasons. The more efficient designs are that which change stiffness with a variation of the lever arm concept, like AWAS [91]. Our design will be based in this lever arm concept. 
Table 3.1: Actuators Comparison

\begin{tabular}{|c|c|c|c|c|c|c|c|}
\hline $\begin{array}{l}\text { Actuator/ } \\
\text { Joint }\end{array}$ & $\begin{array}{c}\text { Weight/ } \\
\text { Dimensions }\end{array}$ & $\begin{array}{c}\text { Peak/Constant } \\
\text { Torque }\end{array}$ & $\begin{array}{c}\text { Stiffness } \\
\text { Range }\end{array}$ & $\begin{array}{c}\text { Stiffness } \\
\text { Adjusting Time }\end{array}$ & $\begin{array}{l}\text { Stiffness Adjusting } \\
\text { By/Max. Deflexion }\end{array}$ & $\begin{array}{c}\text { Torque } \\
\text { Measurement }\end{array}$ & Application \\
\hline Migliore's & $\begin{array}{l}\text { Prototype,big } \\
\text { dimensions }\end{array}$ & $\pm 40 \mathrm{mNm}$ & $\begin{array}{l}0.2 \text { to } 0.8 \\
\mathrm{mNm} / \mathrm{deg}\end{array}$ & $\begin{array}{c}\text { Equilibrium } \\
\text { position and } \\
\text { stiffness dependent }\end{array}$ & $\begin{array}{l}\text { Compression/ } \\
\text { Antagonic }\end{array}$ & None & $\begin{array}{c}\text { Joint/non } \\
\text { linear spring }\end{array}$ \\
\hline VSA II & $\begin{array}{l}345 \text { gr. } \\
\text { Aprox. like } \\
\text { CD-diameter }\end{array}$ & $\pm 2 \mathrm{Nm}$ & $\begin{array}{l}\simeq 0 \text { to } 8 \\
\mathrm{Nm} / \mathrm{deg}\end{array}$ & $\begin{array}{c}\text { Equilibrium } \\
\text { position and } \\
\text { stiffness dependent }\end{array}$ & $\begin{array}{l}\text { Compression/ } \\
\text { Antagonic }\end{array}$ & None & $\begin{array}{l}\text { Joint/4 bar } \\
\text { linkages }\end{array}$ \\
\hline AMASC & $\begin{array}{l}\text { Aprox. } 4 \mathrm{~kg} \text {. } \\
1 \text { meter leg } \\
\quad \text { (large) }\end{array}$ & \multicolumn{3}{|c|}{$\begin{array}{l}\text { Stiffness adjusted by pretension. Slow changes at } \\
\text { low forces. Complex knee system configuration, } \\
\text { intended for running legs. }\end{array}$} & $\begin{array}{c}\text { Compression/pulley } \\
\text { System }\end{array}$ & Embedded & Running leg \\
\hline $\begin{array}{c}\text { MACCEPA } \\
2.0\end{array}$ & $2.4 \mathrm{~kg}$ & $\pm 70 / 50 \mathrm{Nm}$ & $\begin{array}{c}5-110 \\
\mathrm{Nm} / \mathrm{deg}\end{array}$ & $2.6 \mathrm{~s}$ & Compression & Parallel sensor & $\begin{array}{l}\text { Knee Joint/ } \\
\text { rehabilitation }\end{array}$ \\
\hline VS-Joint & $2 \mathrm{~kg}$. & $\pm 180 \mathrm{Nm}$ & $\begin{array}{c}0-5.5 \\
\mathrm{Nm} / \mathrm{deg}\end{array}$ & $0.2 \mathrm{~s}$ & $\begin{array}{l}\text { Compression/ } \\
\pm 14 \mathrm{deg}\end{array}$ & Parallel sensor & Arm Joint \\
\hline QA-Joint & $2 \mathrm{~kg}$. & $\pm 40 \mathrm{Nm}$ & $\begin{array}{l}0.4 \text { to } 9.6 \\
\mathrm{Nm} / \mathrm{deg} \\
9 \mathrm{Nm}\end{array}$ & $0.15 \mathrm{~s}$ & $\begin{array}{l}\text { Compression/ } \\
\quad \pm 15 \mathrm{deg}\end{array}$ & Parallel sensor & Arm Joint \\
\hline CompAct & $1.8 \mathrm{~kg}$. & $\pm 40 \mathrm{Nm}$ & $\begin{array}{c}\text { Damping } \\
\text { 0- Rigid }\end{array}$ & $0.2 \mathrm{~s}$ & Pivot Displacement & Parallel sensor & Joint \\
\hline AwAS-II & $\begin{array}{c}1.1 \mathrm{~kg} . \\
\text { W. } 140 \mathrm{~mm}\end{array}$ & $\pm 80 \mathrm{Nm}$ & $\begin{array}{l}\text { Zero } \\
\text { to rigid }\end{array}$ & $0.8 \mathrm{~s}$ & $\begin{array}{l}\text { Pivot Displacement/ } \\
\quad \pm 17 \mathrm{deg}\end{array}$ & Parallel sensor & Knee Joint \\
\hline ARES & $\begin{array}{c}1.3 \mathrm{~kg} . \\
\text { W. } 80 \mathrm{~mm}\end{array}$ & $\pm 76 \mathrm{Nm}$ & $\begin{array}{l}0 \text { to } 15 \\
\mathrm{Nm} / \mathrm{deg}\end{array}$ & $0.25 \mathrm{~s}$ & $\begin{array}{l}\text { Pivot Displacement/ } \\
\quad \pm 23 \mathrm{deg}\end{array}$ & Embedded & $\begin{array}{l}\text { Multiple } \\
\text { Knee and } \\
\text { Ankle }\end{array}$ \\
\hline
\end{tabular}




\subsection{Conclusions and control challenges}

The strategies of variation of rigidity for the reduction of the energy consumption have been used in bipedal robotics from many different perspectives. Some mechanisms block the knee in hyperextension, increasing its stiffness to the maximum. In this way, the knee is controlled in the support phase without the energy cost of bearing the load[71]. The same mechanism is use in different joints, for example, blocking the ankle when getting up or climbing stairs. In the same way, the characteristics of the actuators can be used with gearboxes to make it suitable for walking. As shown in Section 3.3, the drawbacks of electric motor are inertia and friction. With a gearbox transmission is included this inertia and fiction produced a lack of drivability that could be used to lock a joint and support a load with low energetic cost. On the opposite side, a clutch that disengage the joint of the engine, reducing the stiffness to zero, could be used when limb swings freely.

Another strategy to make conventional actuators more suitable for walking is to include springs in serial and in parallel. These designs introduce energetic improvements that complicates theoretical control design. Clutches and mechanical latches introduce nonlinearities that are incompatible with classical control theory, as they introduce discontinuities. Walking itseft have discontinuities during the gait cycle, being a very nonlinear task. The springs although it has a continuous dynamics, it introduces more states and variables to control.

When controlling a robot with joints of variable stiffness, both principles of passive dynamic walking and the ZMP control have to be taken into account. The designs of passive bipeds produce a stable walking, with a complete lack of control of feedback. But these robots are designed only for the gait that provides the mechanical design, without being able to vary the characteristics of the gait. On the other hand the principles of control of the ZMP are based on the principles of the control of classic feedback. These types of robots do allow a modification of the characteristics of gait are able to adapt to sloping terrain and performing other tasks such as going up or down stairs. Nonlinear control strategies provide analytical methods for the dynamic characteristics of walking. The most promising approaches are: 
1. Virtual model control[92] introduces a representation of a simple model as a pendulum or a spring for the realization of a virtual control in a more complex model.

2. Hybrid zero dynamics [93] applies virtual kinematic constraints to reduce the equations that are applied when walking its dimensionality and thus reduce complexity to improve stability and the robustness.

3. Controlled symmetries[94] studies the passive gait of descending ramps to apply it in the gait on flat ground.

4. Energy shaping[95] uses a dimensional reduction in the walking equations to provide energy only in some parts of the gait, adapting the passive gait from descending ramps to flat terrain and even ascent of slopes.

These approximations have some drawbacks, for example the hybrid zero dynamics involves the use of high gains in feedback which implies a high energy consumption [96].

In the techniques like controlled symmetries and energy shaping are designed to modify the swing at different speeds but in the support phase does not use any strategy other than block or follow a trajectory with high feedback gain.

This thesis proposes an event-based hybrid control joining continuous control intervals. This control model has been implemented in the variable stiffness actuator ARES. 

Practice must always be built on good theory.

Leonardo da Vinci

\section{Analysis of Energy Efficient Gait Approaches For Exoskeletons}

\subsection{Introduction}

In the previous chapters, the strategies for generating energy-efficient human gaits have been outlined. Besides, strategies and techniques to reduce energy consumption in biped robot motion have been analysed.

The analysis and conclusions obtained in Chapter 2 on the biomechanics of human gait in relation to energy consumption, show the versatile behaviour of muscle tendon units:

- In pre-swing and the initial swing the ankle accumulates and releases energy in the muscle-tendon groups.

- In mid-swing in the knee and in the mid-stance in the hip, the joints move freely taking advantage of the passive dynamics of the limbs, while in the mid-stance the knee is almost completely blocked. 


\section{ANALYSIS OF ENERGY EFFICIENT GAIT APPROACHES FOR EXOSKELETONS}

Whereas, in Chapter 3, it is concluded that the robots with lower energy consumption based on passive dynamics are robots that are not versatile because of their underactuactuation. They can walk a long distance with low energy consumption, but they cannot stop and start walking again, they cannot climb ramps, go up or down stairs or sit up and get up. An active orthosis must allow the user to perform daily life activities. The most versatile bipedal robots, which are able to perform the above mentioned tasks feature a high energy consumption. If a low power consumption is desired while maintaining a grade of versatility for the everyday activities, a tradeoff between the underactuated strategies and the rigid actuation of traditional robotics must be encountered.

In addition, in robotics passive elements are often used to reduce the energy consumption such as springs, to store and release energy, fix or variable damping elements (like magnetorheological dampers) and brakes to block and release joint motion in certain phases of the gait. Therefore, the main model proposed in this thesis is obtained from the convergence between both the strategies obtained from biomechanics of human gait and the robotics developments.

\subsection{Methodology to analyse energy consumption}

In this thesis the following strategies to reduce energy consumption have been selected as a trade off between biomechanics and robotics approaches to be applyed in an active orthosis:

1. Utilise passive pendulum dynamics during swing and stance to exploit the intrinsic dynamics of the limbs is these gait phases.

2. Utilise elastic mechanisms to store and release energy.

3. Lock the knee during the mid-stance so as not to spend not required energy.

4. Provide versatile mobility by the required active degrees of freedom to enable the realization of the main activities of daily life.

The system should be able to provide active torques but in a compliant way taking into account the above considerations. A modulated actuation stiffness as biomechanics of human presents is the focus of this thesis strategies. 
In this chapter, the effectiveness and validity of the underlying hypothesis have been verified in a simulation environment of a user wearing an exoskeleton. Therefore, real masses, lengths, centres of mass and moments of inertia of a patient have been included in the simulation with the values shown in Table 4.1, following the estimations and adjustments given in[97] for the Body Inertial Segment Parameters (BISPs).

Table 4.1: Physical parameter of the simulated a 5 year old patient/user wearing a 8 kilograms exoskeleton.

\begin{tabular}{cccccc}
\hline & Torso, arms and head & Thick & Shank & Foot & Total \\
\hline Mass $(k g)$ & 11.96 & 4.92 & 1.9 & 1.2 & 25 \\
Length $(m)$ & 0.571 & 0.285 & 0.292 & 0.124 & 1.15 \\
\hline
\end{tabular}

To estimate power consumption, an active orthosis has been designed using a robot dynamics simulation package (Yobotics SCS[98]). The Yobotics Simulation Construction Set is a software package to create simulations of mechanical devices, biomechanical systems and robots. It allows working in simulation with complex multi-articular systems and to perform simulations and iterations allowing access to joint positions, velocities, and torques. From this simulation package, the entire articular structure of the human legs has been defined, with the characteristics already mentioned in the Table 4.1. (See Figure 4.1) At the same time, the contact points of the active orthosis with the floor have been defined. Throughout this chapter, different techniques have been proposed to reduce energy consumption, and their implementation in the active orthoses has been analyzed through an optimization of mechanical power approach. 


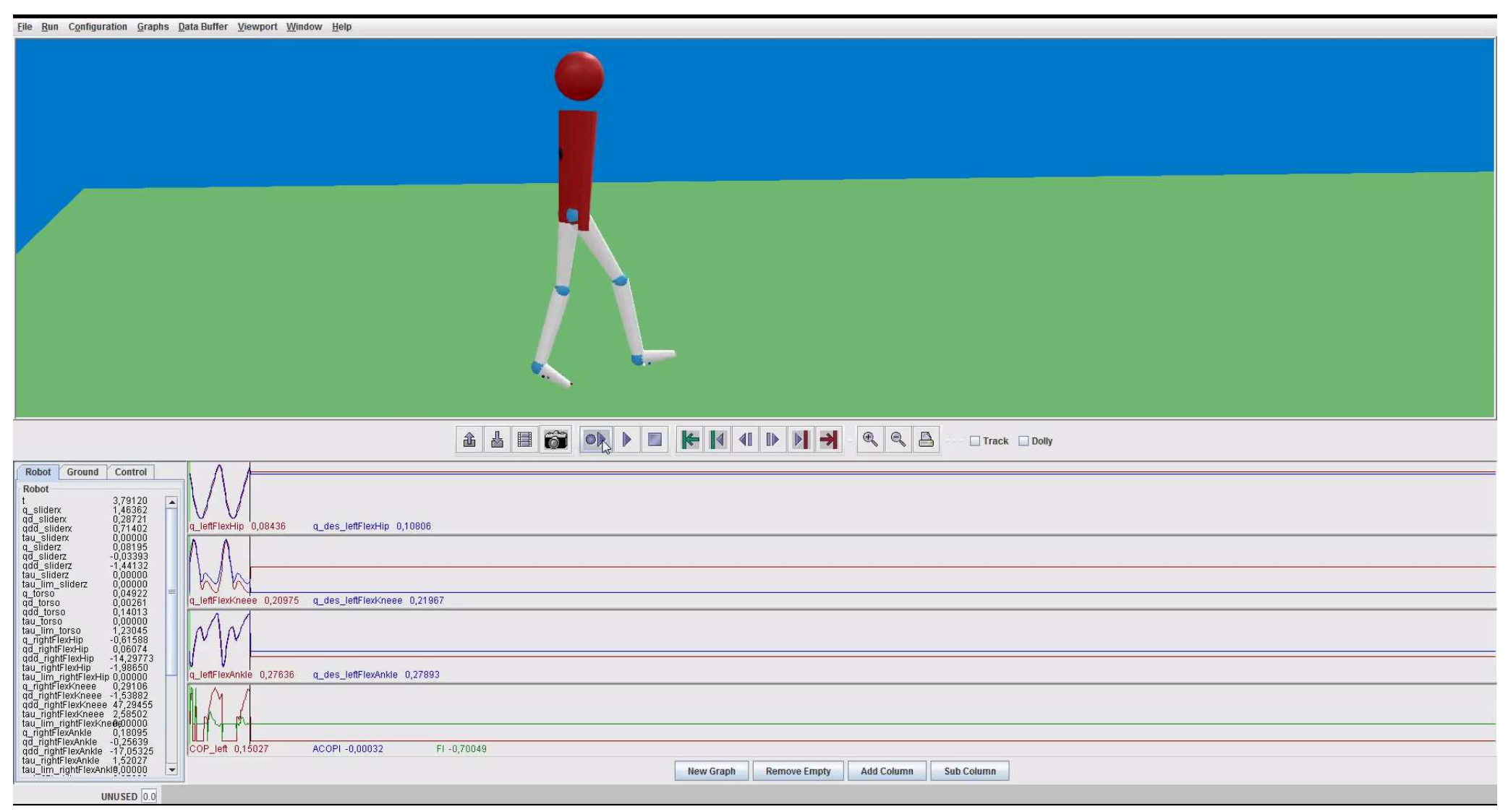

Figure 4.1: User with active orthosis simulated with the Yobotics Simulation Construction Set. 
In addition, to evaluate and quantify the power consumption reduction strategies mentioned above, the power consumption of the simulated active orthosis following a Clinical Gait Analisys (CGA) pattern with a Proportional-Integral-Derivative (PID) control scheme at articular level has been computed. The normalized power consumption (power consumption per unit of mass) obtained in this experiment has been the basis for comparing the results obtained for each of the proposed strategies.

Throughout the chapter an optimization of the energy consumption of the exoskeleton is performed to obtain a gait pattern. In a first approximation a static optimization has been used. In a static optimization, an optimization process is performed in each joint independently, assuming that the optimization of one joint does not affect the rest. It will be observed that, given the number of variables that affect walking, this static method is not good enough, as it does not take into account how the dynamics of the rest of the joints affect the energetics of walking. Due to the synergy between all the leg joints, a reduction of energy consumption in the joint in which the strategy is applied, increases substantially the poewr consumption in the rest of the joints. Then, a heuristic method of genetic algorithms will be used and implemented to tune the parameters in each of the proposed strategies.

\subsection{Measuring gait energy expenditure}

As discussed earlier, the first generation of exoskeleton and active orthoses base their motion control system on the tracking of CGA patterns, usually by implementing a Proportional-Derivative (PD) control strategy. Therefore, some preestablished and normalised angles and velocities, adapted to the height and weight of each user, are used as reference angles and speeds for each actuator, performing a joint torque as follows:

$$
\tau_{\text {joint }}=k_{p}\left(\theta_{\text {real }}-\theta_{C G A}\right)-k_{d}\left(\dot{\theta}_{\text {real }}-\dot{\theta}_{C G A}\right)
$$

where $\theta_{\text {real }}$ and $\dot{\theta}_{\text {real }}$ are the actual measured angle and speed at the joint, $\theta_{C G A}$ and $\dot{\theta}_{C G A}$ are the reference position and speed values, and $k_{p}$ and $k_{d}$ are proportional and derivative gains of the PD control scheme. 


\section{ANALYSIS OF ENERGY EFFICIENT GAIT APPROACHES FOR EXOSKELETONS}

Therefore, the mechanical power consumed in a time $T$ is given by:

$$
P=\frac{\int_{0}^{T} \sum_{i=1}^{2} \sum_{j=1}^{3}\left(\omega_{i j}(t) \tau_{i j}(t)\right) d t}{T}
$$

where $\omega_{i j}(t)$ is the angular speed and $\tau_{i j}(t)$ is the torque at the joint $j$ of leg $i$.

The mean velocity could differ in each experiment. For this reason, a nondimensional parameter is considered for comparison purposes, which is the specific resistance:

$$
\varepsilon=\frac{E}{W L}=\frac{P}{W v}
$$

where $E$ is the energy required to travel a distance $L$ by a vehicle of weight $W, P$ is the power consumed and $v$ is the speed of the vehicle. The specific resistance considers the power consumed per unit of mass and unit of speed.

The strategy of PD controlling CGA joint patterns has been implemented in the simulated active orthosis. $k_{p}$ and $k_{d}$ gains have been tunned but they keep constant during all the gait cycle. The power consumption has been measured through Equation 4.2 and the specific resistance has been calculated through Equation 4.3 . An specific resistance of $\varepsilon=1.13$ has been obtained. This value will be used as a reference for comparison through the rest of the experiments.

Throughout this chapter two types of optimization methods will be presented. A static optimization to each joint separately and a heuristic optimization. This static optimization of each joint separately is not adequate because the joints work synergistically and the change in behavior of one joint affects the functioning of the rest. That is why, then, a heuristic optimization method is studied. In the heuristic optimization the Yobotics SCS package and genetic algorithms are used together to minimize the objective function of the specific resistance, that represents energy consumption, by modifying and adjusting a series of variables, with the simulated active orthosis walking.

\subsubsection{Static optimization method}

This type of optimization is called static because it focuses on one joint and it does not take into account the movement of the other joints. This optimization uses the 


\subsection{Measuring gait energy expenditure}

Nelder-Mead simplex algorithm as described in Lagarias et al. [99]. The NelderMead method is a widely used optimization algorithm. It is a numerical method [100] to minimize an objective function in a multidimensional space. The method uses the concept of a simplex, which is a polytope of $n+1$ vertices in $n$ dimensions: a line segment in a line, a triangle in a plane, a tetrahedron in a three-dimensional space and so on. The method approximates an optimal local solution to a problem with $\mathrm{N}$ variables when the function to be minimized varies smoothly. The algorithm is described as follows:

1. Let $x(i)$ denote the list of points in the current simplex, $i=1, \ldots, n+1$.

2. Order the points in the simplex from lowest function value $f(x(1))$ to highest $f(x(n+1))$. At each step in the iteration, the algorithm discards the current worst point $x(n+1)$, and accepts another point into the simplex. Or, in the case of step 7 below, it changes all $n$ points with values larger than $f(x(1))$.

3. Generate the reflected point

$r=2 m-x(n+1)$,

where

$m=\Sigma x(i) / n, i=1 \ldots n$,

and calculate $f(r)$.

4. If $f(x(1)) \leq f(r)<f(x(n))$, accept $r$ and terminate this iteration.

5. If $f(r)<f(x(1))$, calculate the expansion point $s$

$s=m+2(m-x(n+1))$,

and calculate $f(s)$.

(a) If $f(s)<f(r)$, accept $\mathrm{s}$ and terminate the iteration.

(b) Otherwise, accept $\mathrm{r}$ and terminate the iteration.

6. If $f(r) \geq f(x(n))$, perform a contraction between $m$ and the better of $x(n+$ 1) and $r$ : 


\section{ANALYSIS OF ENERGY EFFICIENT GAIT APPROACHES FOR EXOSKELETONS}

(a) If $f(r)<f(x(n+1))$ (that is, $\mathrm{r}$ is better than $x(n+1))$, calculate

$c=m+(r-m) / 2$

and calculate $f(c)$. If $f(c)<f(r)$, accept $\mathrm{c}$ and terminate the iteration. Contract outside Otherwise, continue with Step 7.

(b) If $f(r) \geq f(x(n+1))$, calculate

$c c=m+(x(n+1)-m) / 2$

and calculate $f(c c)$. If $f(c c)<f(x(n+1))$, accept $c c$ and terminate the iteration. Contract inside Otherwise, continue with Step 7.

7. Calculate the $n$ points

$v(i)=x(1)+(x(i)-x(1)) / 2$

and calculate $f(v(i)), i=2, \ldots, n+1$. The simplex at the next iteration is $x(1), v(2), \ldots, v(n+1)$.

This optimization approach will be executed in a first stage at joint level. In a second stage, the heuristic approach is described and the following section is applied.

\subsubsection{Dynamic parameter optimization}

To perform a dynamic optimization, a method based on genetic algorithms will be applied to the simulation using Yobotics SCS. Each step of the simulated gait cycle is an iteration step of the algorithm in which the objective function (the specific resistance) is calculated, while some other variables, that affect the objective function, are changing to minimize this objective function.

Genetic algorithms are an evolutionary probabilistic search algorithms in which a self-adapted strategy for searching is applied, based on random exploration of solution space joined with a method that enables the algorithms to learn the optimal search path from experience [101].

To facilitate the understanding of the rest of section, the definition of the concepts that will be used are presented in Table 4.2 . 


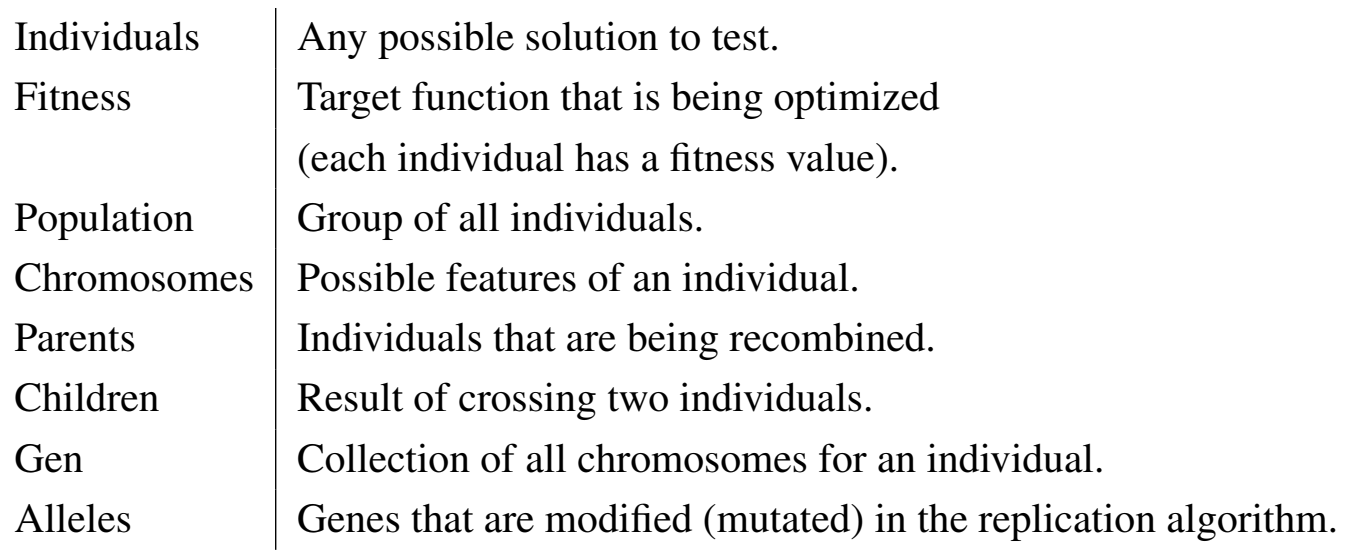

Table 4.2: Glossary of the concepts used in the optimization by genetic algorithms.

In all cases presented in this chapter, the fitness function (cost function) to minimize, related with the energy consumption has been the specific resistance defined in Section 4.3 (eq. 4.3).

The fitness function, specific resistance in our case, depends on some variable or variables. These variables are called "individuals", in our case these individuals will be the stiffness of each joint, and the angle in which this stiffness begins to act.

A fitness value is obtained for each individual. The genetic algorithm looks for the individuals that minimize the fitness value.

These "individuals" must be inside a domain. Each individual is coded with a numerical value, in our case binary, which represents a candidate solution to be tested with the fitness function. We call this coding "chromosome".

Figure 4.2 shows the main concepts used in genetic algorithms. The domain in the individuals is established by defining a maximum and minimum range of the variable to test in the optimization. Chromosome length is chosen, that is, the length in bits of each individual.

A 12-bit chromosome length has been chosen as it is sufficient to obtain accurate results while achieving sufficiently fast convergence. The values of individuals have been assigned with in the minimum and maximum values:

$$
\begin{gathered}
\left.10 \frac{\mathrm{Nm}}{\mathrm{rad}} \rightarrow 000000000000\right|_{2}=\left.0\right|_{10} \\
\left.1000 \frac{\mathrm{Nm}}{\mathrm{rad}} \rightarrow 111111111111\right|_{2}=\left.4096\right|_{10}
\end{gathered}
$$




\section{ANALYSIS OF ENERGY EFFICIENT GAIT APPROACHES FOR EXOSKELETONS}

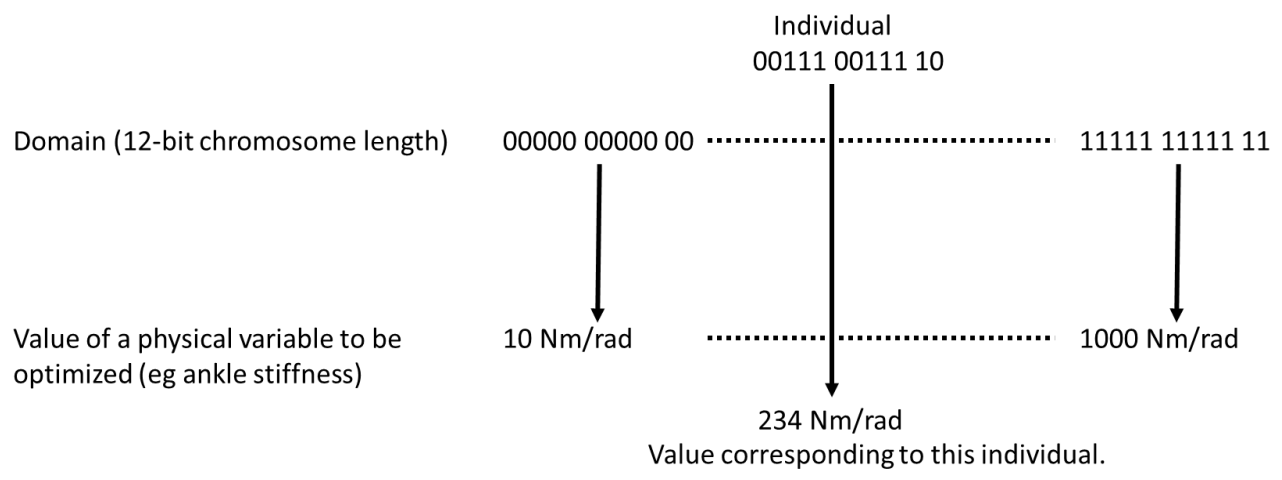

Figure 4.2: Concepts used in genetic algorithms.

respectively. So, the equation that gives the value of the individual in decimal format is:

$$
y=\frac{4096}{1000-10} x-10 \frac{4096}{1000-10}=4.137 x-41.37
$$

For example, if a value of $234 \frac{\mathrm{Nm}}{\mathrm{rad}}$ is assgined to an individual this value correspond in that range to:

$$
4.137 * 234-41.37=\left.926\right|_{10}=\left.001110011110\right|_{2}
$$

This is the individual that will be optimized.

Subsequently a group of individuals, that is a population, is chosen, corresponding to a series of values. The standard genetic algorithm procedure is composed of the following sequence of step(see Figure 4.3).

1. Generate randomly the initial population, with the appropriate number of individuals. To obtain accurate results and at the same time with sufficiently fast convergence, 5 individuals of the population have been chosen.

2. For each individual, the fitness function, specific resistance, is calculated in a complete walking step (from one heel strike to the next).

3. An intermediate population is generated by applying a reproduction operator. 


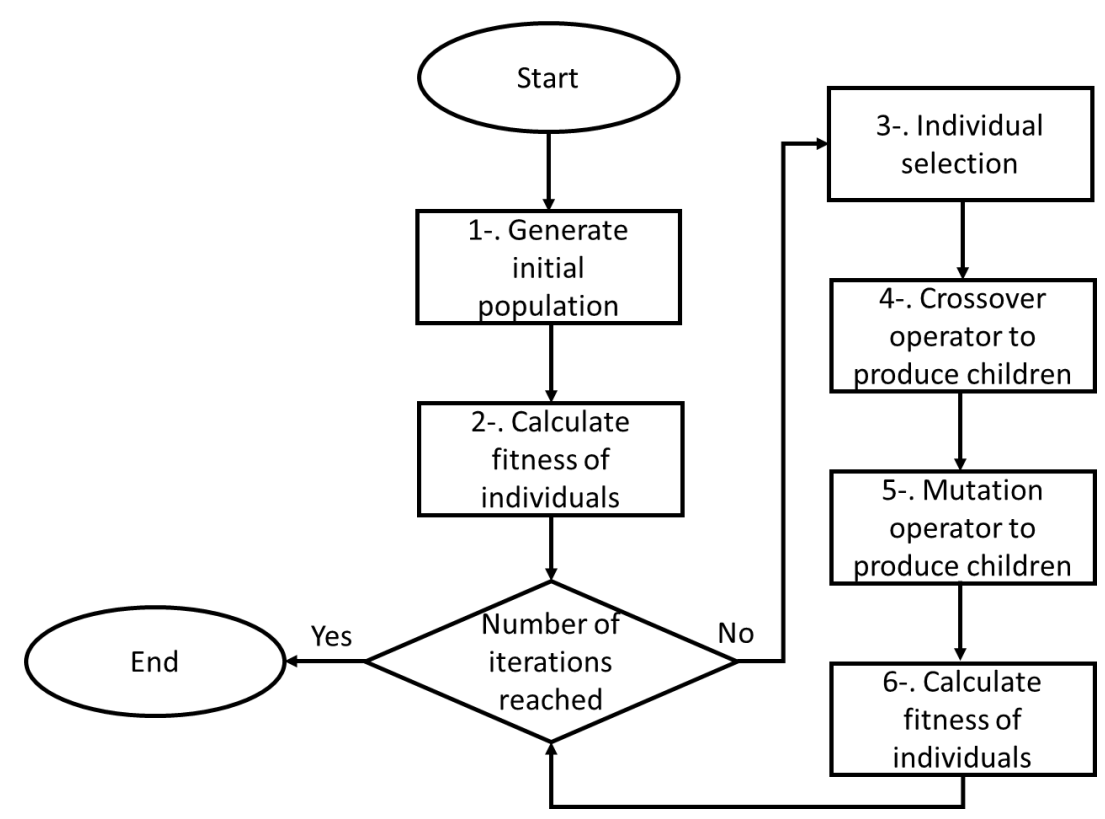

Figure 4.3: Procedure followed in the genetic algorithm applied.

4. This operator selects the elements of the old population that will produce new population. In order to choose the individuals of the old population who pass through this breeding operator, a probabilistic mechanism is used where the probability of extraction of each individual is proportional to their fitness value.

5. Generate a new population by applying some operators to the chosen elements of the old population. To choose the best genes two operators are used, mutation and crossover.

- Mutation operator: Randomly modifies one or more bits (called alleles) of individuals with a uniform probability of distribution. This allows to reach parts of the distribution space of the variable that would not reach directly chosen individuals.

- Crossover Operator: Combines the chromosomes of the progenitor individuals. The position of the bit number where the chromosome of one of the parents is going to be cut is randomly obtained.A piece of a random length of the chromosome of the other parent is introduced at 


\section{ANALYSIS OF ENERGY EFFICIENT GAIT APPROACHES FOR EXOSKELETONS}

this point. An example is: If the randomly obtained recombination bit number is 2 and the chromosome crossing length is 4 (with our 12-bit chromosome length), these children are obtained from these parents.

Parent 101001001010

Parent 010010100010

Child 100010001010

Child 011001100010

6. Repeat the process from step 2 .

Given the chosen population, four steps are required for the crossover operator and another four for the mutate operator.

The value or values obtained through this genetic algorithm is a local minimum over the space of the objective function.

In the next sections of this chapter, static and dynamic optimization is applied to minimize specific resistance in the next instance:

- In the first instance, to take advantage of the passive dynamics of the limbs, the values of hip and knee stiffness that minimize the specific resistance in each step are calculated with these optimization methods.

- Then, the influence on the energy consumption of elastic elements in both the hip and the ankle is analyzed, being the elastic constant of the elements to include and the range of the gait cycle, the parameters to minimize the specific resistance.

- In the same way, with these optimization techniques, it is analyzed how the variation of the damping of the knee influences the energy consumption as well as introducing a blockage in this articulation.

- Finally, the results of applying these optimization strategies are presented, combining the methods of exploitation of passive dynamics with the elastic elements. 


\subsection{Exploiting passive pendulum dynamics during swing and stance}

Based on the pendulum gait model[102] revised in detail in Chapter 2, the inherent swing properties of the leg are taken into account to reduce the energy expenditure while the legs follow the desired trajectory. The gait controller is designed as follows. Once the swing phase begins, the leg continues its trajectory with little energy injection. In the single-support phase of the gait, the biped robot behaves as two coupled pendulums formed by the stance and swing legs. The swing phase is divided into three sub-phases. In the early swing, a constant torque is applied to both the hip and knee joints by means of a ballistic approach until each limb reaches the necessary speed[73]. In the middle of the swing phase, the hip and knee move freely, requiring almost no torque. At the end of the swing phase, the $\mathrm{PD}$ controller is used at the joints to achieve the desired posture at the moment of heel strike.

Given that in order to exploit passive dynamics the influence of the rest of the articulations and the global system is fundamental, performing a static optimization of each independent articulation in this case does not make sense. For this gait control model only dynamic optimization of the specific resistance has been used by genetic algorithms. As already explained the objective function is the specific resistance while to determine are the PD control constants (eq. 4.1): $k_{p h i p}, k_{d h i p}$, $k_{p k n e e}$ and $k_{d k n e e}$.

To implement the gait controller, a simple state machine has been assigned to each leg. For each state, the control constants have been optimized (see Figure 4.5 (a)). In each state of the state machine, proportional and derivative (PD) gains are maintained, while the arrows indicate the event that causes the state change.

Table 4.3 shows the resulting energy consumption when applying this control strategy and the energy saving achieved over the pure PD controlled CGA pattern.

A remarkable reduction in energy consumption of $14.9 \%$ is achieved, with this dynamic optimization.

Analyzing more deeply the dynamics of the leg when walking it is observed that the kinetic energy of each link (thigh and shank) reaches the maximum in different instants of gait cycle (see Figure 4.4). 


\section{ANALYSIS OF ENERGY EFFICIENT GAIT APPROACHES FOR EXOSKELETONS}

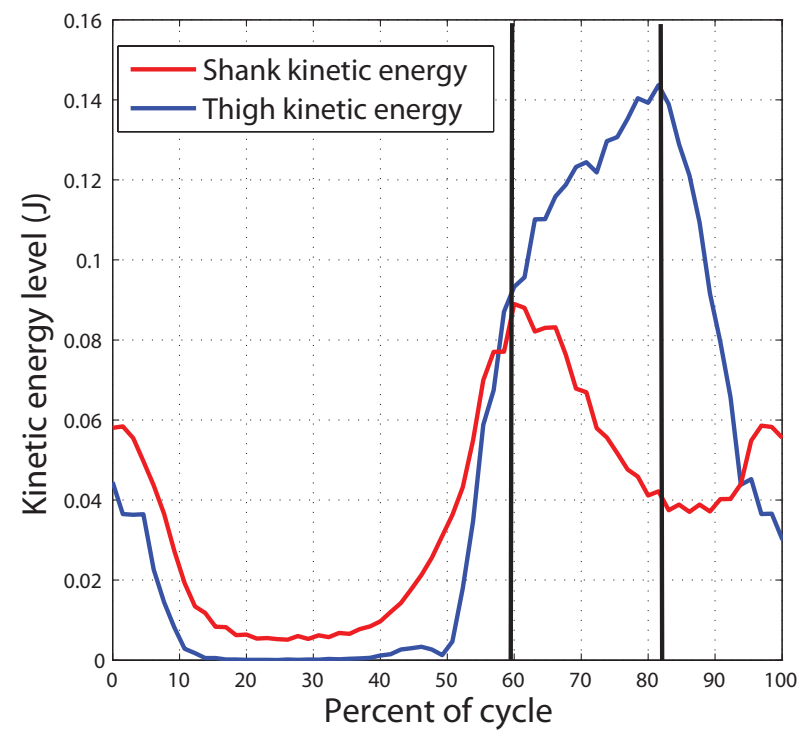

Figure 4.4: Gap in the maximum kinetic energy in thigh and shank.

For this reason, a more efficient control algorithm has been proposed by implementing two separate state machines, one for the hip, shown in Figure 4.5 (a), and the other one for the knee, shown in Figure 4.5 (b). Once again, a dynamic optimization of the PD control constants $\left(k_{p h i p}, k_{d h i p}, k_{p k n e e}\right.$ and $\left.k_{d k n e e}\right)$ has been carried out and the values obtained are shown in Figures 4.5 (a) and (b). Table 4.3 also compares the efficiency of this proposed approach with the one based on a single state machine, showing that with this new strategy a reduction in mechanical energy expenditure of $16.8 \%$ is achieved, improving the previous approach.

Table 4.3: Results of applying a passive control strategy.

\begin{tabular}{lcc}
\hline $\begin{array}{l}\text { Control } \\
\text { strategy }\end{array}$ & $\begin{array}{c}\text { Specific } \\
\text { resistance }\end{array}$ & $\begin{array}{c}\text { Energy saving } \\
\text { reduction } \%\end{array}$ \\
\hline PD Control of CGA references & 1.13 & \\
\hline $\begin{array}{l}\text { A state machine } \\
\text { for hip and knee together }\end{array}$ & 0.97 & 14.9 \\
\hline $\begin{array}{l}\text { Two state machines, } \\
\text { one for the hip and another for the knee }\end{array}$ & 0.94 & 16.8 \\
\hline
\end{tabular}




\subsection{Storing and releasing energy in elastic mechanisms}

This section focused on the active control of the hip and knee to reduce power consumption. The ankle can also be controlled through passive elements that provide ground clearance. This will be analysed in the next section.

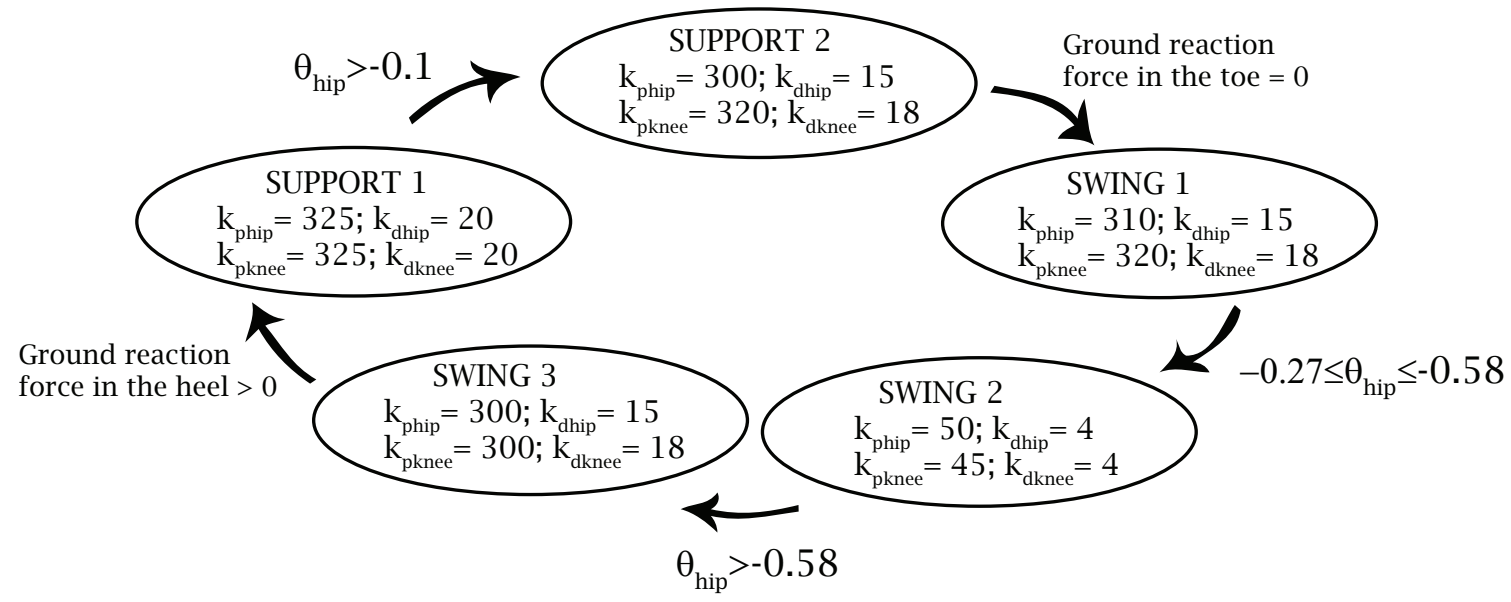

(a)

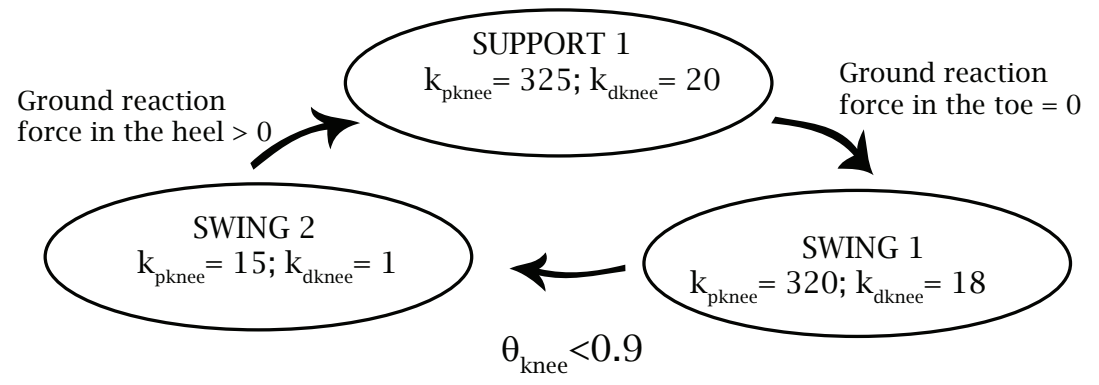

(b)

Figure 4.5: State machine controller. In each state, the PD constants and the transition condition are shown (a) for hip and knee and (b) for the knee

\subsection{Storing and releasing energy in elastic mechan- isms}

Human tendons are passive elastic elements that store energy and release it without metabolic cost. In the ankle joint, which shows the highest peak of the power requirements, more than $60 \%$ of the energy required for locomotion is transferred by tendons[103]. 


\section{ANALYSIS OF ENERGY EFFICIENT GAIT APPROACHES FOR EXOSKELETONS}

By analysing the biomechanical features of the human gait, as it was presented in Chapter 2, it was concluded that energy consumption can be reduced through the use of passive elastic elements. From the study of a low-speed walking gait it is shown that the hip and the ankle joints have periods of negative power followed by a period of positive power. A spring could be used to store energy during the negative power period and release it during the positive power period of these two points. Moreover, power at the knee is predominantly negative, meaning that it primarily dissipates energy.

\subsubsection{Storing energy at hip and ankle}

The storage of potential energy has been analysed by including springs at the ankle and the hip in the simulation of the active orthosis. From the torques $\tau$ and the angles $\theta$ in these joints obtained from the CGA, the mechanical power computed from equation (4.2) has been minimised, taking into account that a torsion spring exerts torque if the ankle joint exceeds a threshold angle $\theta_{\text {thres. }}$. This torque is given by:

$$
\tau_{\text {spring }}=\left\{\begin{array}{cl}
k_{\text {spring }}\left(\theta-\theta_{\text {thres }}\right) & \text { if } \quad \theta>\theta_{\text {thres }} \\
0 & \text { if } \quad \theta<\theta_{\text {thres }}
\end{array}\right.
$$

where $k_{\text {spring }}$ is the spring constant, $\theta$ the joint angle and threshold angle $\theta_{\text {thres }}$.

Applying the static optimization approach presented in Section 4.3.1. optimal values for the spring constants $k_{\text {spring }}$ and threshold angle $\theta_{\text {thres }}$ are obtained and shown in table 4.4 (a).

In this static optimisation, it is assumed that the kinematics of the gait do not change when including passive elements. However, when these springs are introduced in the simulated biped, it is observed that although the power consumption is reduced in the joint in which the spring has been added, the rest of the joints are stressed, and the overall power expenditure increases. Departing from the statically optimised values, a dynamic adjustment based on genetic algorithms, detailed in Section 4.3.2 has been applyied to the values for spring constants $k_{\text {spring }}$ and threshold angle $\theta_{\text {thres }}$. The optimized values with the objective function of the specific resistance are shown in Table 4.4 (b).

Figure 4.6 shows torque vs angle at the hip and ankle joints in a gait cycle. The torque exerted with a pure CGA position control approach is shown in red. The 
Table 4.4: Values for spring constants $k_{\text {spring }}$ and threshold angle $\theta_{\text {thres }}$ in hip and ankle joints.

(a) From static optimisation

\begin{tabular}{|c|c|c|}
\hline Joint & $k_{\text {spring }}(N m / r a d)$ & $\theta_{\text {thres }}(\mathrm{rad})$ \\
\hline Hip & 120 & -0.28 \\
\hline Ankle & 230 & -0.05 \\
\hline & \multicolumn{2}{|c|}{ (b) From a genetic algorithm dynamic optimization } \\
\hline Joint & $k_{\text {spring }}(N m / r a d)$ & $\theta_{\text {thres }}(\mathrm{rad})$ \\
\hline Hip & 200 & 0.05 \\
\hline Ankle & 80 & 0.05 \\
\hline
\end{tabular}

torque when a spring is included is shown in green and the torque exerted only by the spring is shown in black.

Table 4.5 shows the reduction in energy consumption obtained when incorporating a spring at the hip, at the ankle and at both joints simultaneously. Note the significant reduction in energy expenditure when springs at the hip and ankle are working together, demonstrating the synergy between these joints.

Some conventional prosthesis already incorporate elastic elements in the ankle, however the energy stored is not released spontaneously in the right moment of the toe-off phase. (See this effect in Figure 4.7). This is because the elastic elements are passive devices, not able to control the instant of activation, resulting in a premature release of recycled energy. In the prosthesics presented in [8] a mechanisms that releases energy at the exact moment of the ankle push-off is incorporated basee on the human biomechanics, there by consuming $10 \%$ less energy than with the use of a espontaneusly released spring.

In this work, the springs incorporated into the simulated active orthoses are also spontaneously released. Therefore, the results presented in this section will be improved for a lower energetic consumption by actively controlling the stiffness of the joints in each phase of the gait. 


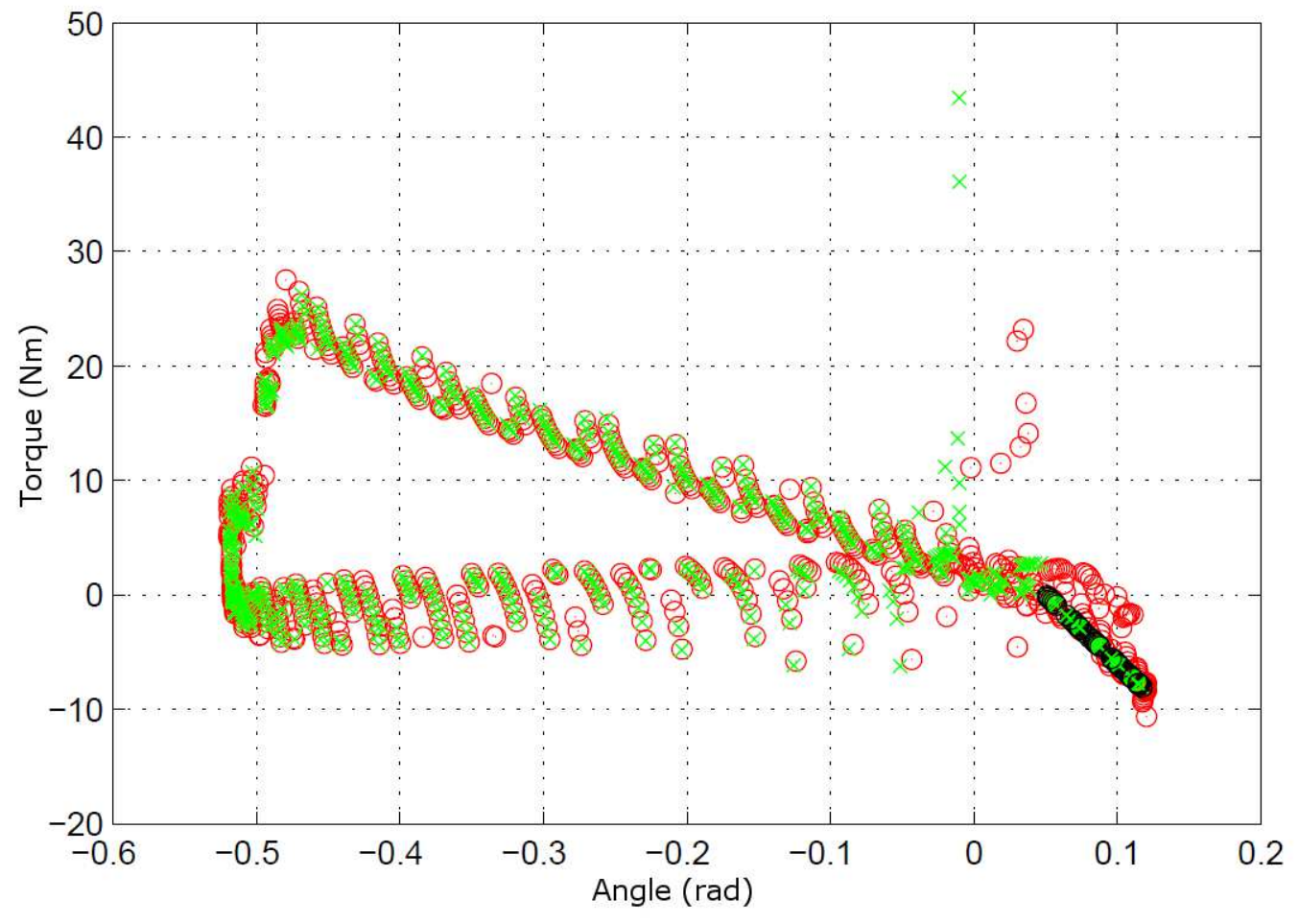

(a)

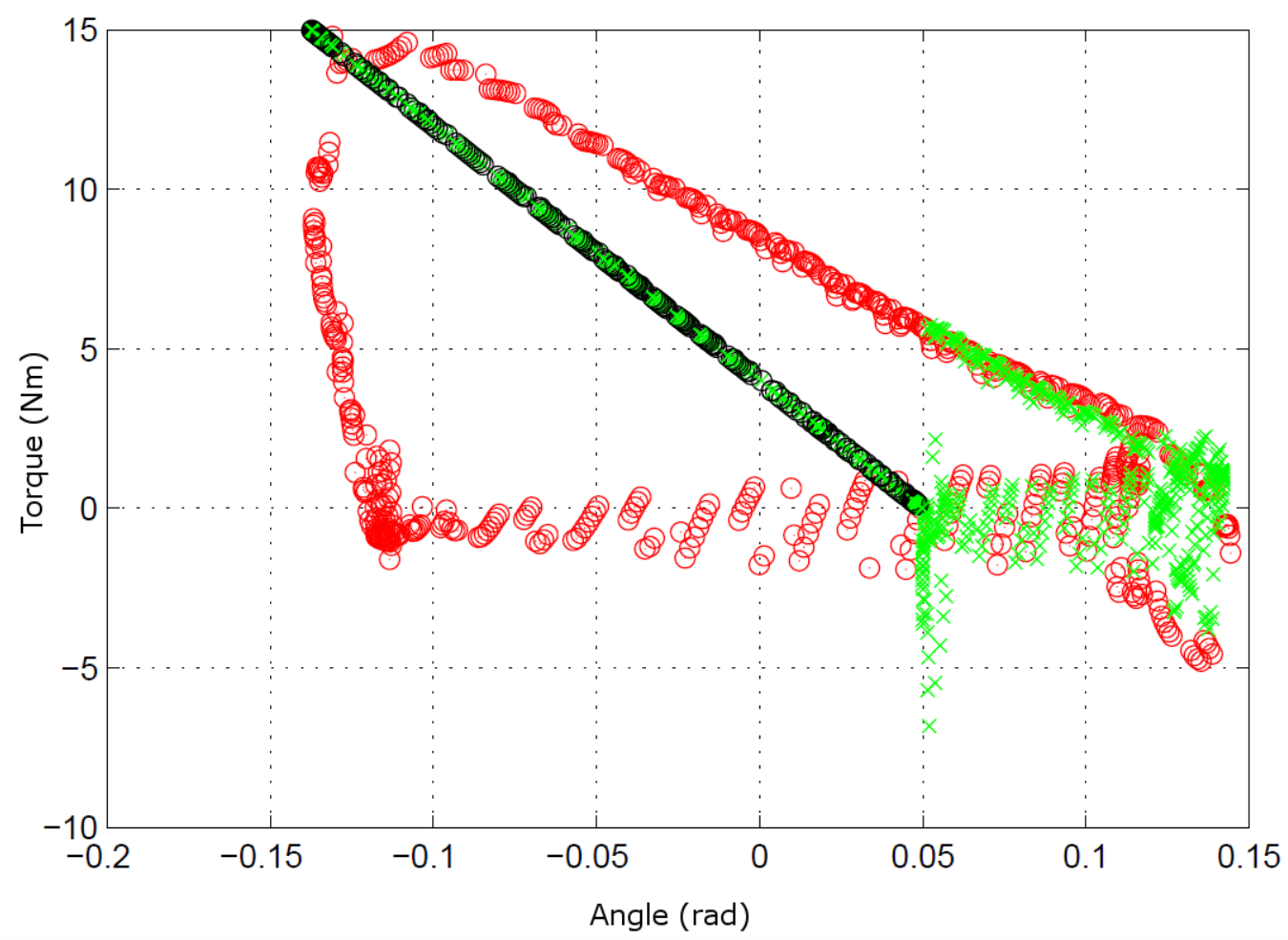

(b)

Figure 4.6: Torque vs angle in: (a) Hip and (b) Ankle. The torque exerted with a pure CGA position control approach is shown in red. The torque exerted only by the spring is shown in black and the torque exerted when a spring is included is shown in green. 
A Conventional Prosthesis

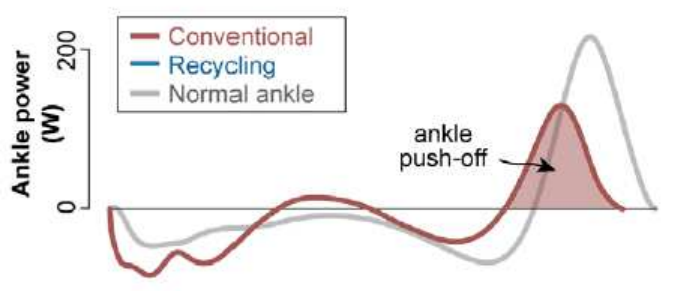

B Energy Recycling foot

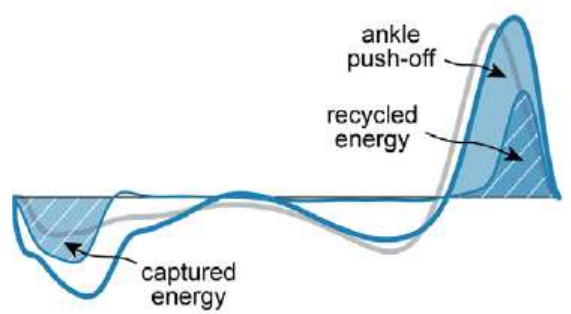

Figure 4.7: (A) The power in Conventional Prosthesis is released too early in the push-off phase. When a mechanisms is inlucled (B) to hold the energy and released in the right moment the redirection of the center of mass is more efficient. From [8].

\subsubsection{Knee damping}

As previously mentioned in Chapter 2, the knee works dissipating energy during most of the gait cycle. The use of a dissipative device like a damper could help save energy during knee braking. The torque exerted by a damper is, not taking hysteresis into account:

$$
\tau_{\text {damper }}=\left\{\begin{array}{cl}
B \dot{\theta} & \text { if damper is enabled } \\
0 & \text { if damper is disabled }
\end{array}\right.
$$

where $B$ is the damping constant and $\dot{\theta}$ is the joint angular speed.

As in the previous subsection, to determine the energy efficiency of the approach, an static optimisation has been performed with the algorithms of Section 4.3.1 based on the human CGA angles. After applying this static optimisation, the value of the damping constant that obtains a minimum in the specific resistance is $B=3.8 \mathrm{Nms} / \mathrm{rad}$ in the pre-swing phase, in the range of the gait cycle from $55 \%$ to $64 \%$. When the damping coefficient is increased during the pre-swing phase, the foot will not achieve ground clearance, and the simulated biped stumble against the ground, making the static theoretical results of the damping misleading. Therefore, the damping constant and the range of application in the gait cycle have been again optimized now using a dynamically approach with genetic algorithms. After applying this dynamic optimization a energy reduction of $1.2 \%$ is obtaing, with a constant damping of $B=4 \mathrm{Nms} / \mathrm{rad}$ during the $15 \%$ to $35 \%$ portion of the gait 


\section{ANALYSIS OF ENERGY EFFICIENT GAIT APPROACHES FOR EXOSKELETONS}

cycle. Table 4.5 shows the results of including passive elements to a passive control strategy.

Table 4.5: Results of including passive elements to a passive control strategy.

\begin{tabular}{lcc}
\hline $\begin{array}{l}\text { Control } \\
\text { strategy }\end{array}$ & $\begin{array}{c}\text { Specific } \\
\text { resistance }\end{array}$ & $\begin{array}{c}\text { Energy } \\
\text { reduction } \%\end{array}$ \\
\hline PD CGA Control & 1.13 & \\
\hline Hip spring & 1.11 & 1.7 \\
\hline Ankle spring & 1.04 & 7.2 \\
\hline Knee damping & 1.11 & 1.2 \\
\hline Locking the knee & 1.07 & 4.8 \\
\hline Hip and ankle springs & 0.96 & 14.48 \\
\hline Hip and ankle springs and locking the knee & 0.91 & 18.9 \\
\hline
\end{tabular}

The analisys presented in Chapter 2, when analyzing the damping requirements at the knee, suggests that the knee damping coefficient during the gait cycle is variable. One recent solution is the use of magnetorheological fluids (MRF) devices that can modify its damping coefficient dynamically. Previous studies[50] that use magneto-rheological damping at the knee, have not achieved energy reduction and have actually increased metabolic rate consumption. The causes for this increment are the additional weight of the passive devices which increase inertia and a non zero introduced friction from the magneto-rheological damper in off-state producing energy losses. The same result was obtained by applying a magnetoreological device to the knee joint of a robotic leg in [104]. An improved design of MRF device is needed to be included in an active orthosis in order to reduce energy consumption, the current state of the technoloy is not adequate to the requerement of this appication.

\subsubsection{Knee locking}

By examining the human gait, it is observed that in some phases, the knee angle is almost constant (see Chapter 2). A lock has been considered for blocking the knee when the swing leg moves forward and when the other leg is in the singlesupport phase, as some passive robots do [71]. Adjusting the activation range of 
the locking system via genetic algorithms, it is shown that the lock can be engaged in the terminal stance phase from $30.3 \%$ to $40.2 \%$ of the gait cycle and in the mid swing from $67.1 \%$ to $85.2 \%$ of the gait cycle. This locking knee results in an energy consumption reduction of $4.8 \%$.

After analysing the benefits of each passive element (springs, damping and brakes), an analysis is performed to consider the combined energy-saving benefits of all these mechanisms.

\subsection{The combined approach}

In an active orthosis for disable patients that are unable to walk, all the power must be provided by the actuators. With the goal of reducing energy consumption as much as possible, a combined strategy of passive walikng with added passive elements is analyzed. For this porpose, the control pattern described in Section 4.4 will be used with a finite state machine, but now the response in each sub-phase will be changed by adding the passive elements described in Section 4.5.1.

In phase SUPPORT 2 (see Figure 4.5), most of the work required from the actuator is supplied by the spring at the hip, so no actuator torque is necessary. In the middle and last phases of the swing (phases SWING 2 and 3 in Figure 4.5), a passive walikng scheme is applied to take advantage of the thigh inertia.

At the same time, if a knee damper is included in early stance, then a state machine can be implemented at mid-swing to exploit the potential energy stored, thus reducing the actuator power consumption.

Figure 4.8 shows the finite state machines employed for the incorporated hip and knee passive elements.

Energy expenditure is reduced by $24.6 \%$, achieving a specific resistance of 0.85 when passive elements are combined with a strategy of passive walking. Table 4.6 presents a comparison of the specific resistance of several bipeds [55]. From an energetic point of view the obtained results are located closer to passive robots like Cornell Biped than to actively controlled robots like ASIMO. This comparison should be analyzed carefully taking into consideration that our results are based on simulations and not on a real experiments where mechanical efficiency and friction 


\section{ANALYSIS OF ENERGY EFFICIENT GAIT APPROACHES FOR EXOSKELETONS}

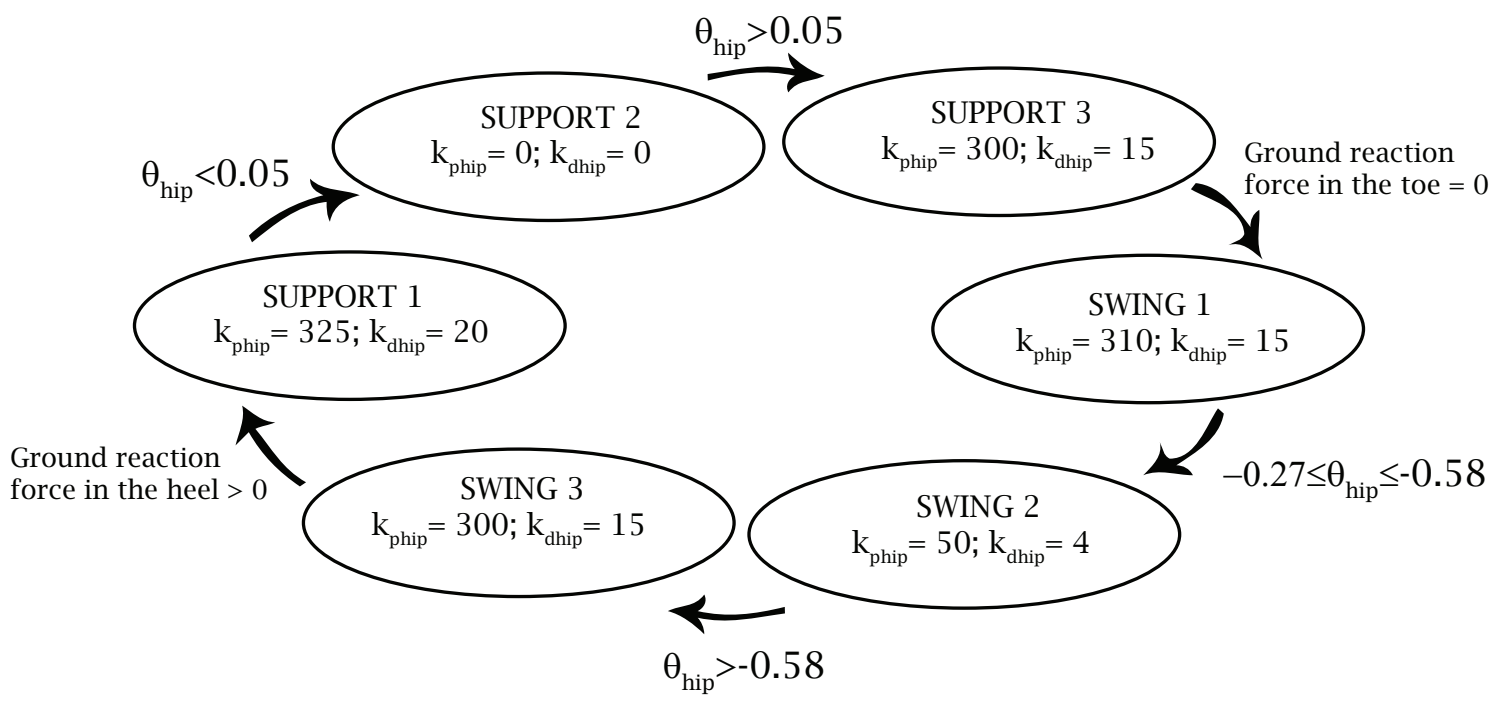

(a)

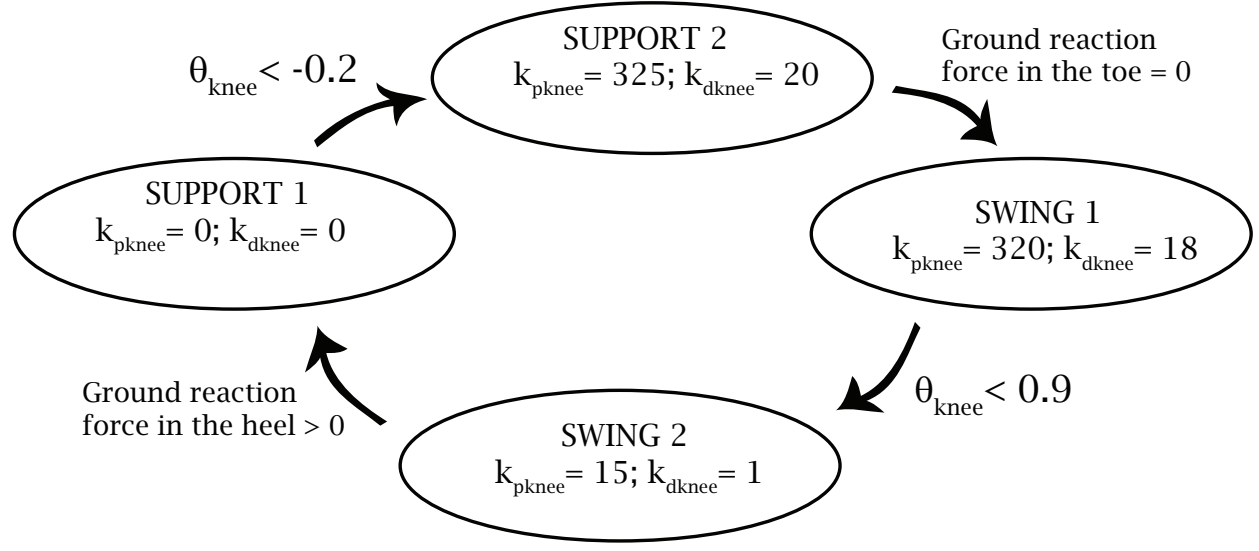

(b)

Figure 4.8: Finite state machine with passive elements: (a) At the hip ; (b) At the knee 
increase the specific resistance. Moreover, different robots have different actuated degrees of freedom which affects its power consumption.

Table 4.6: Comparison of specific resistance in several bipeds.

\begin{tabular}{lc}
\hline Biped & Specific Resistance \\
\hline Delft Pneumatic Biped & 5.4 \\
\hline Honda ASIMO & 3.2 \\
\hline ATLAS simulation & 0.85 \\
\hline Cornell Biped & 0.28 \\
\hline Man & 0.2 \\
\hline
\end{tabular}

\subsection{Feasibility of the presented proposals}

This chapter shows in a simulated bipedal robot how the modification of the stiffness of the joints affects the total energy consumption. The technique of minimization of genetic algorithms has been applied to calculate the rigidity and damping that minimize energy consumption for the hip, knee and ankle joints. This supposes a contribution of this thesis to the state of the art.

First, genetic algorithms are applied to calculate the rigidity of the hip and knee joints that achieve a minimum in specific resistance, with this technique a reduction in energy consumption of $16.8 \%$ is achieved.

Subsequently, it is analyzed how the inclusion of passive elements in joints whose torque reflects a linear behavior with respect to the position, such as a spring, influences in energy consumption. Thus, the inclusion of a spring in hip and ankle is simulated and the springs constants and the instants of the gait cycle where these elements act to minimize the specific resistance are calculated. After this minimization a reduction in energy consumption of $18.9 \%$ is achieved.

Finally, the genetic algorithm approach is applied in a combination of these previous techniques, the passive elements and exploiting passive dynamic. In this way, a reduction in energy consumption of $24.6 \%$ is achieved.

To incorporate these approaches into reducing energy expenditure in gait execution, some points must be taken into account: 


\section{ANALYSIS OF ENERGY EFFICIENT GAIT APPROACHES FOR EXOSKELETONS}

1. An active orthosis must be designed as a whole; electrical and mechanical systems must be integrated to achieve a specific motion control.

2. Rigid actuators are not ideal for walking with real-world physical interactions

3. The proposed motion control approach, in which kinetic and potential energy is exploited and springs are used to store and release energy, is not achievable with rigid actuators.

Therefore, the proposed strategy in this chapter requires the ability to adjust joint stiffness. In the range from zero stiffness to exploit inherent dynamics at swing to large stiffness to lock the motion during stance.The concept of robots changing the stiffness of their joints is not new [84] and some variable stiffness actuator designs have been proposed [79]. However, in the design of a wearable active orthosis, the size and weight of the actuator is a main design parameter. Therefore, some of the developments rely on pneumatic compliance controller designs[105], which are not feasible for active orthosis because of the need for an air compressor and a distribution system. Other proposed mechanically controlled approaches, such as AMASC at Carnegie Mellon University [106], MACCEPA at Vrije Universiteit Brussels[107], VS-joint and QA-joint mechanisms at the German Aerospace Centre(DLR) [108], or AwAS at the Italian Institute of Technology (IIT)[88] are too large or heavy for the required torques. Closer examination of the results obtained in this study (see Figure 4.8) shows that a continuous range of stiffness is not necessary for a walking orthosis. Discrete stiffness values at different phases are required instead. In the approach proposed in this thesis, it is determined that only three stiffness states are necessary:

1. Free limb motion, to take advantage of the inherent dynamics of the limbs, required negligible torque: null stiffness.

2. Spring recoil, to store and release energy when a phase of negative power is followed by one of positive power. A specific spring stiffness is needed to exploit this feature, depending on the joint, the speed of walking, and the weight of the user.

3. Locking of gear motor motion: infinite stiffness. 
None of the above state-of-the-art variable stiffness actuators are able to provide these three states. In Chapter 6 a new variable stiffness actuator is presented, developed in the frame of the ATLAS project to implement the energy-efficient gait approach proposed in this chapter. 

The essence of synergy is to value differences-to respect them, to build on strengths, to compensate for weaknesses.

\subsection{Introduction}

In the previous chapters, certain strategies that converge to achieve a low energy consumption have been analyzed both from the biomechanics point of view and from the robotics point of view. In this chapter, another feature of human muscleskeleton system, the synergy between joints will be analized and exploited. Exploiting joint synergy takes into account two features in the design of exoskeletons, very relevant from the point of view of energy consumption:

- Reducing the number of actuators of the exoskeleton: The number of actuators used is a very important parameter to take into account in the design of a lower limb active orthosis. The number of actuators must be sufficient to allow a stable walking but not too high because each new actuator adds an extra weight that significantly affects the power consumption aditional to the own power consumed by the motor. Some research orthoses, such as the Vanderbilt Orthosis [109], and commercial devices, such as the Rewalk [44] active orthosis and eLEGS [46] are not actuated on the ankle, and some of 


\section{SYNERGIC MULTIARTICULAR ACTUATION}

these devices include only a passive element to provide ground clearance, an elastic element (usually a spring) that stores energy in the initial phases of stance and releases it in the push-off phase. Some of them incorporate an actuator but not to move the joint [8], but to keep the spring compression and release it in the right moment of the gait and to let a correct redirection of the COM of the body, as it has been explained in the previous chapters.

- Reducing the weight in the distal limbs. From the physical point of view, placing a lot of mass on the links farthest from the center of mass will cause the inertial torque to increase considerably during swing, especially at high walking speeds. The contribution in energy expenditure of the swing phase is more significant than was predicted, recent studies suggest that the cost of swinging the limbs can reach up to $25 \%$ of the cost of locomotion [110]. Several experimental studies show the high cost associated with adding load to the more distant limbs [111].

On the other hand, in order to achieve a more efficient and natural gait it is necessary to actuate the ankle. The addition of an actuator or passive element increases weight of the limbs and consequently the moment of inertia of the leg, resulting in larger power consumption in hip and knee joints. In the theoretical work by Van der Bogert [112], an energetic optimization is performed, joining hip and ankle by an exotendon and achieving a $47 \%$ reduction in joint power consumption.

Taking this analysis into account, in this chapter, a mechanism that resembles a human tendon to achieve and take advantage of synergic actuation of leg joints is presented. A tendon has been attached at the ankle acting like a synergic biarticular linkage and transferring motion from hip and knee joints. In this way, high inertia in the more distal limbs is prevented and, at the same time, power to actuate the ankle joint is provided. This mechanism has been integrated in a prototype of lower-limb active orthosis, actuated at hip and knee joints. Taking advantage of hip and knee rotations and thanks to the attached tendon, a movement in the ankle joint is induced, that has to be mapped to a CGA ankle joint pattern to achieve a natural motion. After implementing this strategy in the active orthosis, an experimental analysis has been performed. The experiment has been focused in the toe-off, swing and heel-strike phases, which are considered the most determinants 
of gait energetics[56]. The fitted ankle flexion and extension helps the leg motion during the toe-off phase, provides ground clearance by preventing foot dragging during the swing phase, and accommodates for a proper heel-strike, resulting in a more natural gait.

\subsection{Ankle motion along the gait cycle}

Figure 5.1 shows ankle, knee and hip angles of a healthy person, obtained from CGA. To follow the ankle joint behavior of the CGA pattern, the gait cycle has been divided into three phases. The first phase corresponds to the support phase, where

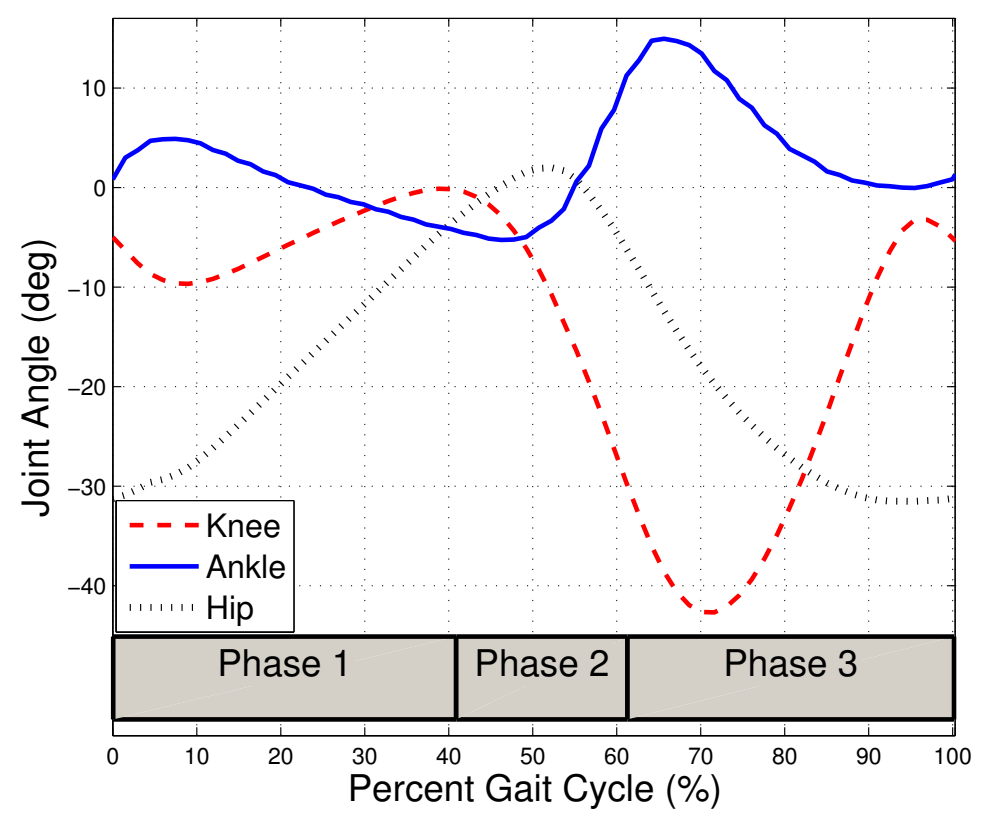

Figure 5.1: Gait cycledivided into three phases: 1-. Stance, 2-. Toe-off and 3-. Swing

the foot is accommodated by the ground, which acts as a mechanical constraint, so in the beginning of this phase no tendon will act.

In the second phase, that corresponds to the final part of support, the toe-off phase, the trailing leg gives impulse before the leading leg begins heel strike. From an energetic point of view this is the determinant phase of the gait [56]. Analyzing the behavior in this toe-off phase, it is seen that the ankle has the inverse behavior 
of hip and knee, see Figure 5.1. Taking advantage of this fact, attaching a tendon joining the hip to the foot through the knee, passively provides the adequate motion torque to the ankle.

Once the foot has left the ground, phase 3 begins, that correspond to the swing phase. In this phase, the ankle movement has to provide ground clearance and accommodate the foot for heel strike. In this third phase, see Figure 5.1, the ankle has the same behavior than hip, so another tendon will take advantage of the motion of the hip to move the ankle.

\subsubsection{Providing toe-off impulse}

In the mentioned gait second phase, to provide the required impulse in the ankle at toe-off, a tendon has been attached, joining the hip to the foot through the knee. Analyzing Figure 5.2 it follows that the ankle angle is related to hip and knee angles by:

$$
\Delta\left(\theta_{\text {ankle }}\right) \propto R_{\text {ankle }}^{-1}\left[R_{\text {hip }} \Delta\left(\theta_{\text {hip }}\right)+R_{\text {knee }}\left(\theta_{\text {knee }}\right) \Delta\left(\theta_{\text {knee }}\right)\right]
$$

where $R_{\text {ankle }}, R_{\text {knee }}\left(\theta_{\text {knee }}\right)$ and $R_{\text {hip }}$ are the active pulley ratios, calculated to achieve an adequate movement in the ankle.

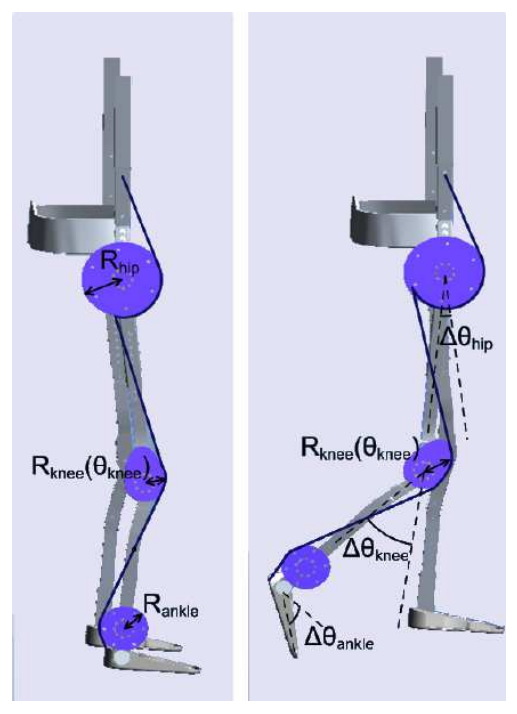

Figure 5.2: Ankle angle depends on the motion in hip and knee. 
It can be observed that the hip pulley ratio $R_{h i p}$ is larger than the ankle pulley ratio $R_{\text {ankle }}$ because a small variation in $\theta_{\text {hip }}$ has to provide an important variation in $\theta_{\text {ankle }}$. An eccentricity has been added to the knee pulley so the active ratio $R_{\text {knee }}\left(\theta_{\text {knee }}\right)$ is not constant and it depends on the knee angle. Thus, the impulse of the trailing leg is given at the proper time. Several simulation adjustments have been made for different active ratios to give the impulse in the right moment in the cycle, see Figure 5.3 (a).

\section{Adjusting pulley radios in the toe-off}

Analyzing the variation of the angle of the joints in the pre-swing phase (from $50 \%$ to $60 \%$ of gait cycle), there is a variation of $\Delta\left(\theta_{\text {hip }}\right)=6.1^{\circ}$ in the hip, $\Delta\left(\theta_{\text {knee }}\right)=$ $23.2^{\circ}$ in the knee and $\Delta\left(\theta_{\text {ankle }}\right)=-24^{\circ}$ in the ankle.

Introducing these values in the previous equation (eq. 5.1) and rewriting $K_{1}=$ $\frac{R_{h i p}}{R_{\text {ankle }}}$ and $K_{2}=\frac{R_{\text {knee }}}{R_{\text {ankle }}}$, it is obtained:

$$
-24=K_{1} * 6.1+K_{2} * 23,2
$$

With this restriction, providing impulse in the toe-off phase, this equation (eq 5.2) with two unknown quantities is obtained, so to calculate the radii of the pulleys, another equation is need. This equation is obtained in the next section with another restriction in the swing phase.

\subsubsection{Providing ground clearance in the swing phase}

Another tendon takes advantage of the motion of the hip to move the ankle, providing ground clearance in the third gait phase, the swing. At the beginning of this swing phase, the plantar flexion presents its higher angle while the hip has already started the transition to swing the leg. The larger hip active ratio reduces the tension of the first tendon preventing the interlock and compensating rapidly the action of the knee eccentricity. The attached tendon causes that when $\theta_{\text {hip }}$ increases, advancing the leg, $\theta_{\text {ankle }}$ also increases, lifting the foot to clear the ground and preparing for heel strike. Again, active ratios have been adjusted to achieve the correct ankle motion, see Figure 5.3 (b). In this case, no eccentricity is needed in the knee. 


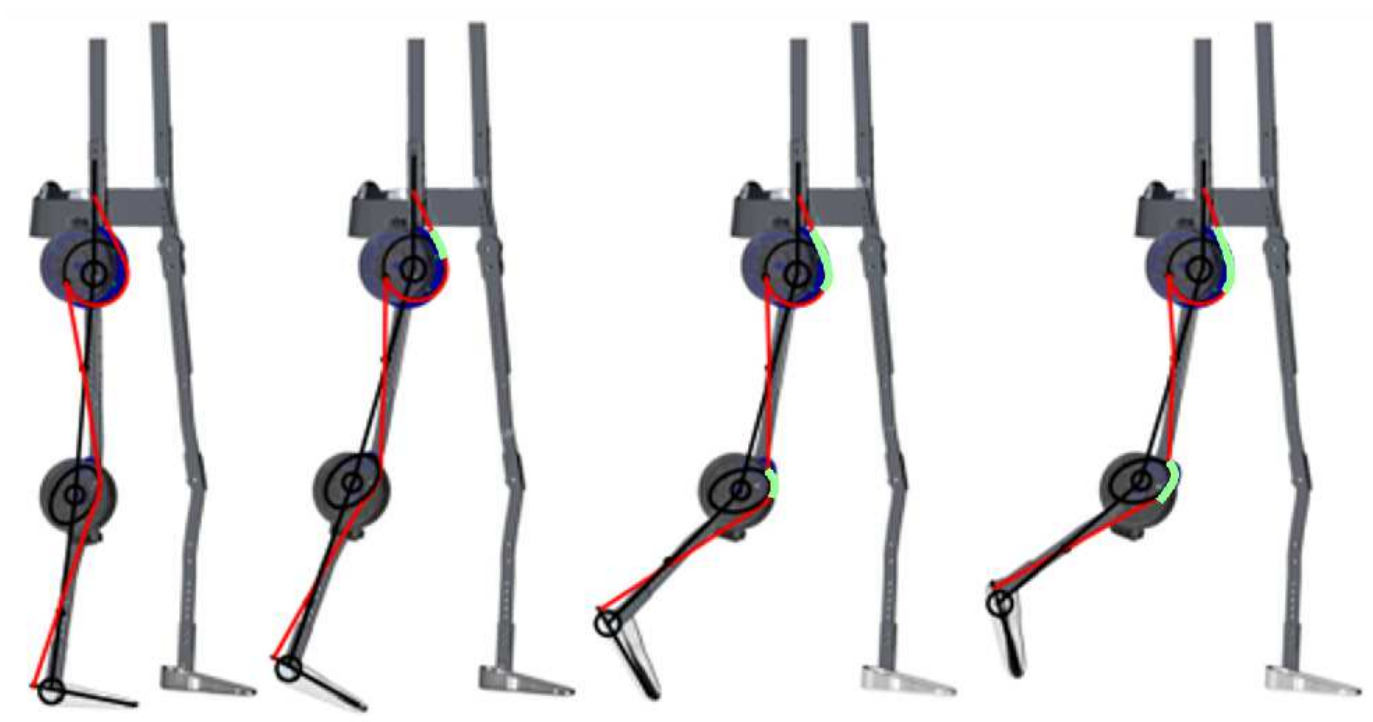

(a)
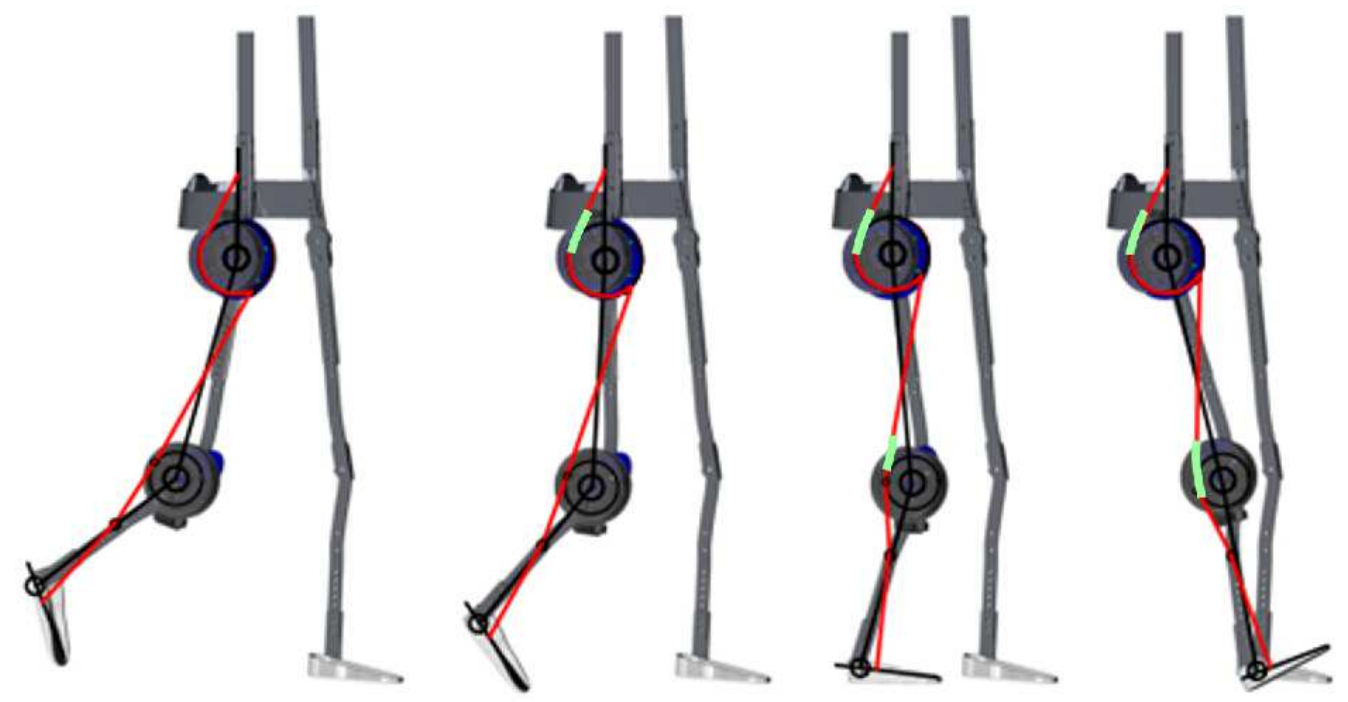

(b)

Figure 5.3: Tendons attached to move the ankle (a) A tendon provides the impulse in the ankle. (b) Another tendon provides ground clearance in the swing. 


\section{Adjusting radios at the swing phase}

Analyzing the variation of the angle of the joints in the swing phase (from $65 \%$ to $77 \%$ of gait cycle), there is a variation of $\Delta\left(\theta_{\text {hip }}\right)=30^{\circ}$ in the hip, $\Delta\left(\theta_{\text {knee }}\right)=6.8^{\circ}$ in the knee and $\Delta\left(\theta_{\text {ankle }}\right)=14^{\circ}$ in the ankle.

When these values are introduced in the equation 5.1 and again with the notation $K_{1}=\frac{R_{\text {hip }}}{R_{\text {ankle }}}$ and $K_{2}=\frac{R_{\text {knee }}}{R_{\text {ankle }}}$, it is obtained:

$$
14=K_{1} * 30+K_{2} * 6,8
$$

Solving the system equation 5.2 and 5.3 , the values of $K_{1}$ and $K_{2}$ are obtained:

$$
K_{1}=\frac{R_{\text {hip }}}{R_{\text {ankle }}}=0.24 \quad \text { and } \quad K_{2}=\frac{R_{\text {knee }}}{R_{\text {ankle }}}=0.97
$$

This equation provides adequate hip and knee active radii for a given $R_{\text {ankle }}$.

\section{The need of a knee pulley eccentricity}

When the variation of angle during the stance phase (from $10 \%$ to $40 \%$ of gait cycle) at the hip $\operatorname{Delta}\left(\theta_{\text {hip }}\right)=-32.8^{\circ}$, at the knee $\Delta\left(\theta_{\text {knee }}\right)=-11.7^{\circ}$ and at the ankle $\Delta\left(\theta_{\text {ankle }}\right)=15.5^{\circ}$ is included in the equation $(5.1)$, the following is obtained:

$$
15.5=-K_{1} * 32.8-K_{2} * 11.7
$$

Maintaining the value of $K_{1}=0.24$ obtained in equation (5.4), and introducing it in the equation 5.5 , a value of $K_{2}=\frac{R_{\text {knee }}}{R_{\text {ankle }}}=0.65$ is obtained.

From these equations, a synergic performance is achieved throughout the gait cycle, with a constant value in the hip and the ankle of:

$$
R_{\text {hip }}=8 \quad \text { and } \quad R_{\text {ankle }}=1.92
$$

However, in the knee differing values are needed at the stance phase and at the toe off. From equations (5.2), (5.3) and (5.5) the values of the knee pulley ratios obtained are:

$$
\begin{array}{ll}
R_{\text {knee }}=1 * R_{\text {ankle }}=1.92 & \text { at toe }- \text { off } f \text { and } \\
R_{\text {knee }}=0.65 * 1.92=1.25 & \text { at stance phase } .
\end{array}
$$


In order to allow these different radii of curvature calculated at different gait phases, a eccentricity has been included in the knee of the prototype as shown in the Figure 5.3 .

\subsection{Prototype implementation}

An active orthosis prototype ATLAS has been used to assess experimentally the exotendon concept. Two tendons have been connected through the joints of the powered orthosis and a goniometer has been attached to the shank and foot for measuring the ankle angular variation. The active ratios that have been calculated in simulations for every joint, have been incorporated in the ATLAS prototype orthosis using pulleys (see Figures 5.4 (a) and (b)). Notice that both tendons in the mechanisms cannot act simultaneously. This multiarticular actuation system avoids the backdrivability of the joints. The ankle angle excursion has been evaluated, while CGA patterns are commanded. Figure 5.5 shows snapshots of a gait cycle in

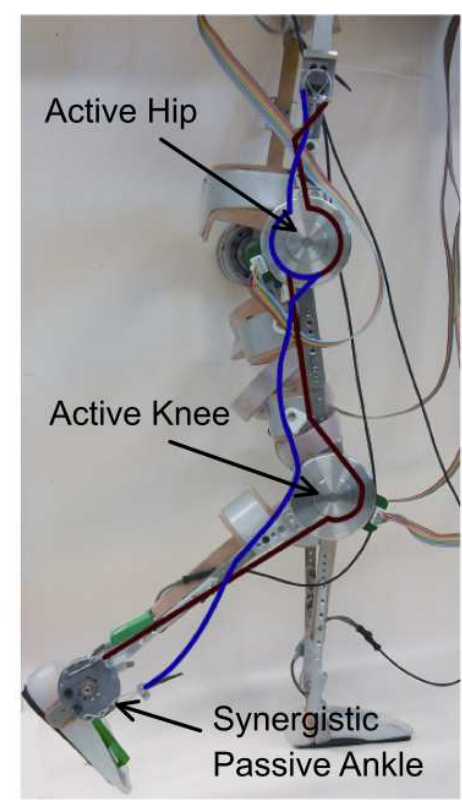

(a)

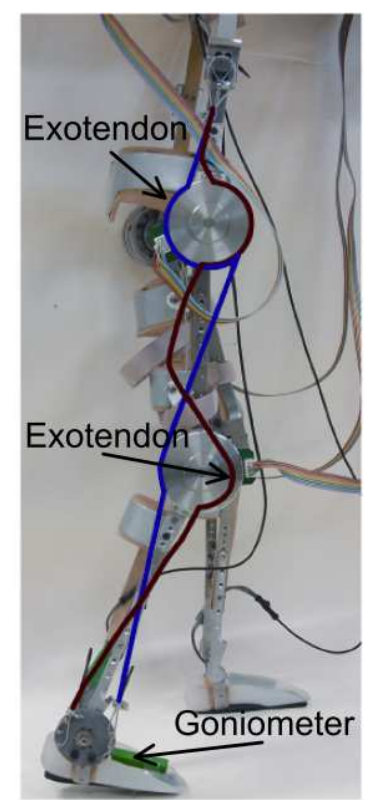

(b)

Figure 5.4: Exotendons in ATLAS active orthosis prototype: (a) Red tendon providing toe-off impulse; (b) Blue tendon providing ground clearance. Tendon paths have been drawn by lines in the pictures to clarify the illustration. 
real experiments. The passive synergic ankle motion provides impulse in the toeoff, increases support force in stance and helps providing ground clearance. The analisis of the resulting gait pattern is presented in the next section.

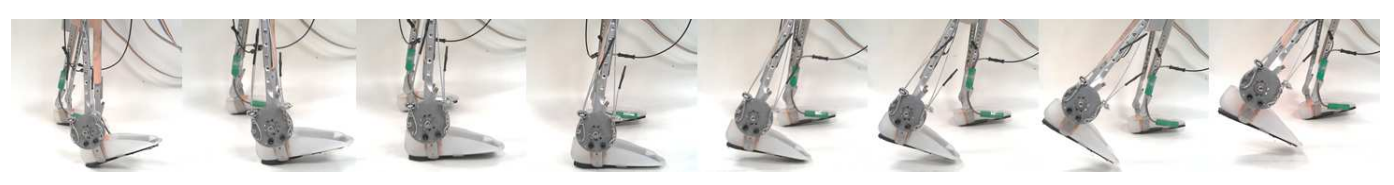

(a)

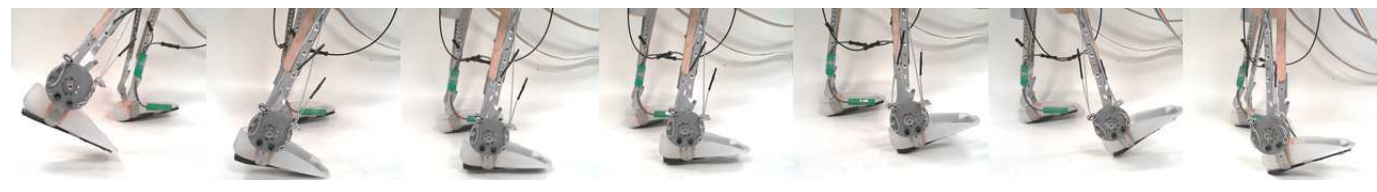

(b)

Figure 5.5: Ankle movement based on exotendons in a gait cycle: (a) Right leg support phase and toe-off; (b) Right leg swing phase.

\subsection{Experimental evaluation}

As it is explained in Appendix A, instead of following fixed walking pattern, in ATLAS active orthosis, parametric trajectory step have been implemented in each supporting or swinging leg. In the supporting leg, an inverted pendulum trajectory has been parameterized to achieve a specific step length and in the swinging leg a direct pendulum trajectory has been parameterized to ensure an adequate step height and strike angle[113]. Therefore, these parametric trajectories provide a safer, configurable and more versatile walking pattern. Then, the hip and knee trajectories do not match exactly CGA pattern. Figure 5.6 shows the angles commanded to the actuators in hip and knee, and the angle measured by the goniometer at the ankle. The experiments have been done with the orthosis walking on the ground and holding only to keep balance. The analisys is as follows, based on the gait phases defined in 5.2 (see figure 5.1):

- Phase 1: Support phase, the foot acts as a mechanical constraint, so the ankle does not move only provides rigidity. 
- Phase 2: Toe-off phase, the trailing foot ankle provide impulse.

- Phase 3: Mid-swing, the ankle provide ground clearance.

\subsubsection{Leg behavior in phase 1}

In this first phase the supporting leg moves from heel strike to the beginning of the toe-off impulse. The foot is in contact with the ground in most of this phase of the gait and there is little demand of ankle torque, just to adapt to the ground allowing the movement of hip and knee joints. In the proposed multiarticular actuation scheme, the tension of the tendons to provide ankle angular positions throughout this phase is low, providing compliance to the ankle and therefore the mechanical constraint imposed by the ground contact determines the variation of the ankle angle. In Figure 5.6 a small variation of the angle can be seen, just after a little bump corresponding to the heel strike. This bump can be bigger, depending on the amount of ankle joint restriction. For this experiments a $-5^{0}$ degrees of dorsiflexion are allowed in the mechanical structure.

\subsubsection{Leg behavior in phase 2}

The tendon that provides plantar flexion has been stretching during phase 1 . When the hip passes the neutral position $\left(\theta_{h i p}=0\right)$ and starts extension, the tension of the tendon increases sharply. This is caused by the larger active ratio of hip joint in combination with a variation of the knee joint angle and therefore an increase in the knee active ratio. The ankle angle then yields the plantar flexion, as shown in Figure 5.6. This movement provides impulse to the gait at toe-off making it more natural.

\subsubsection{Leg behavior in phase 3}

The flexion of the hip has been accelerated in the experiment, as Figure 5.6 illustrates. Instead of providing all the ground clearance with the ankle movement, an increase in the knee flexion along the swing phase is provided. The ankle presents dorsiflexion even when the knee angle is still increasing, this is because of the lower 
contribution of the active ratio of the knee eccentricity compared to the active ratio of the hip, as was intended in the design. At the end of this phase the foot is entirely extended and with a correct position for heel strike.

\subsection{Conclusions and application remarks}

For an active orthosis application, weight and size are main considerations. A synergic multiarticular actuation for the ankle joint, implemented by two exotendons in each leg, improves the functionality of the device without increasing the leg weight and while maintaining a reduced size and weight. These exotendons act as a synergic biarticular linkage and transfer motion from hip and knee joints to the ankle. Synergic ankle motion provides impulse in the toe-off, increases supporting force, and provides ground clearance. These goals are achieved with low energy expenditure because of reduced actuation, and high inertia is prevented in the distal limb. The selection of the pulleys and their shape provided a versatile actuation system for the three studied gait phases. The different sizes and shapes of pulleys produce different active ratios that, in accordance with the hip and knee joint angular variations, can accelerate the ankle movement and reduce or increase the tendon tension, thereby making the ankle joint compliant or stiffer depending on (and avoiding) the interlocking of the tendons.

The synergic actuation proposed in this chapter is very suitable for a continuous gait on even terrain. However, it would not be suitable to sit or stand up, because the movement of the ankle has a different relationship with the hip and knee compared to that of the gait. Therefore, this synergic biarticular design for the ankle motion will only be feasible if it can be coupled and uncoupled in different types of motion.

However, any development that is intended to be implemented in a active orthosis, whether passive dinamic components, a synergical bisticular exotendon or any other arrangement, must be sufficiently versatile and reliable to provide motion in the daily life activities of the user, such us walking, sitting down, standing up, and climb stairs.

For this reason, multiarticular actuation proposed is shown to be a very effective idea for the reduction of energy consumption saving one actuator per leg, but usable only for a continuous walk. Versatility still requires direct actuation at the ankle. 


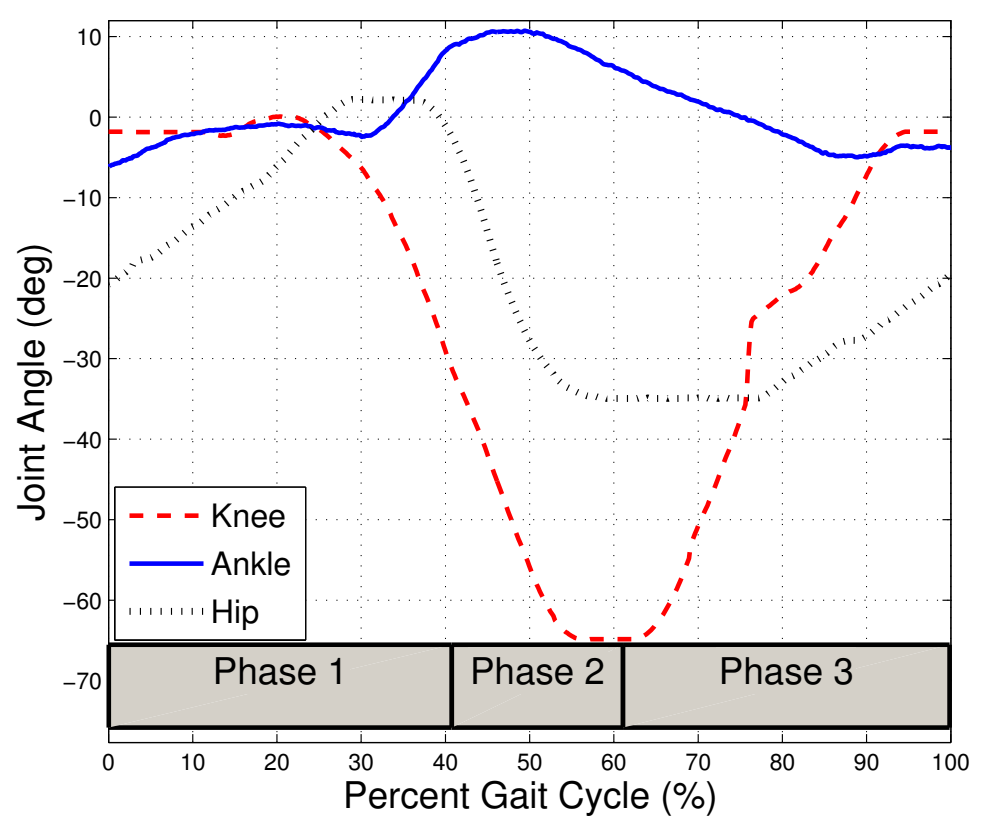

Figure 5.6: Angles at the exoskeleton's right leg joints in real experiment.

The focus in the reminder of this thesis will be on achieving actuators that are able to reduce energy consumption by exploiting the dynamics of the legs and accumulating and releasing energy. Therefore, in the following chapters the ankle will be considered, together with the knee and hip, independently active with the proposed new actuator designs.

In Chapter 6, two variable impedance actuators are proposed, designed and tested. These actuators allow to exploit the double pendulum model ideas, while provide safety features in the rest of activities. 
The measure of a person's strength is not his muscular power or strength, but it is his flexibility and adaptability.

Debasish Mridha

\section{Variable Stiffness Actuation}

As concluded in Chapter 5 a new paradigm of compliant actuation and control of active orthoses is needed, but not only for energetic reasons, also for safety in human-robot interaction. Changing the stiffness of the joints to adapt to the symptomatology of the patient is a need. Usually, users of active orthoses suffer spastic or spasmodic movements, that the orthotic device must absorb, which makes a compliant actuator absolutely necessary. The stiffness variation can be imitated by software through impedance control techniques[114] with a stiff actuator, but due to a limited bandwidth it is not possible to absorb shocks, and energy would be dissipated continuously, being energetically expensive.

A variety of adjustable compliant actuators [79] has been designed due to the demand of actual robotics regarding safe human-robot interaction and energy-efficiency. Series Elastic Actuators (SEA) are composed of an electric motor that transmits the motion through a set of spring. Therefore, SEA has an inherent mechanical lowpass filter, which makes these actuators safer in human environments. Furthermore, elastic elements can store energy and release it, reducing external energy supply.

However, the current state of the art in the field of compliant joints was not designed for werable lower-limb exoskeletons, and they do not fulfill all of the requirements of this application. 
The actuators used in lower-limb exoskeletons require a reduced volume and weight and high power-to-weight ratio. So they usually are designed with a relatively small and light weight motor and gearbox with large reduction ratio which provides the required torque and speed at the output. But this motor-gear set has the disadvantage of being too rigid. Moreover, these actuators should comply with size, weight and power requirements while providing compliance to the joints.

A new adjustable compliant actuator has been designed and developed in the frame of the ATLAS project[115]. It extends longitudinally along the leg so that it does not protrude. Its design is based on a stiff conventional actuator in which adjustable compliance has been included. In this chapter, actuator features are determined based on active-orthoses requirements, and operation is analyzed.

\subsection{Joint requirements for active-orthoses}

In this section, the maximum torque and velocity values in the joints of the sagittal plane measured in healthy children are defined, based on data extracted from articles [9] and [116]. Figures 6.1, 6.2 and 6.3, show the angular movement and the net momentum of the joints in the sagittal plane when walking at normal speed $(1.29 \pm 0.23 \mathrm{~m} / \mathrm{s})$ on level ground. Note that these data were obtained from children aged 6 to 17 years, on average $10.8 \pm 3.2$ years; Weight $41.4 \pm 15.5 \mathrm{~kg}$; Height $1.47 \pm 0.20 \mathrm{~m} ; 9$ boys and 11 girls, which fits with the data of the target patient.

Let's analyze each joint separately as each one has different torque and velocity requirements.

\subsubsection{Actuator requirements at the hip}

As shown in the Figure 6.1 of hip flexion data, the maximum absolute torque required is $0.5 \mathrm{Nm} / \mathrm{kg}$, in the phases in which the joint changes its direction. The maximum weight of the target user is $32 \mathrm{~kg}$ plus an exoskeleton weight of $19 \mathrm{~kg}$, making a total of $51 \mathrm{~kg}$. Thus the nominal maximum torque required is $25.5 \mathrm{Nm}$.

The maximum angular velocity is $35 \mathrm{rpm}$. It is important to note that the phases of higher torque requirement match the lower speed phases. 


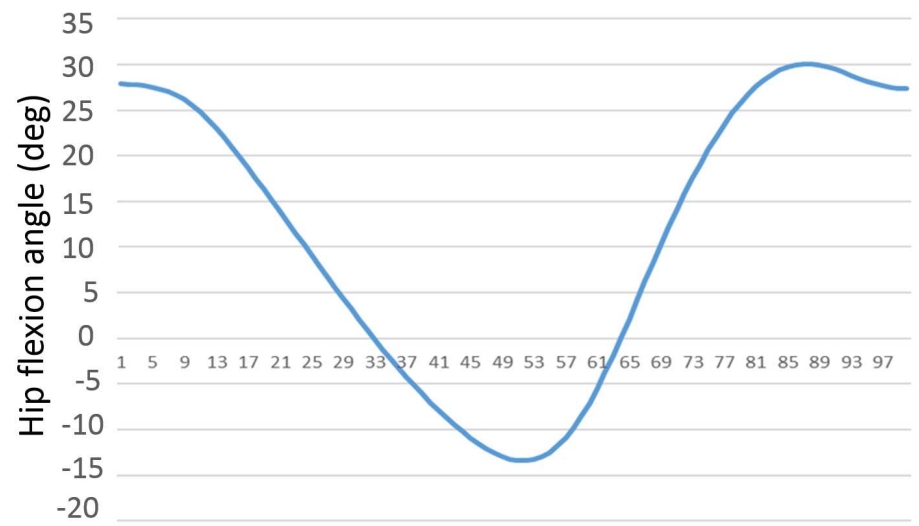

Gait cycle (\%)

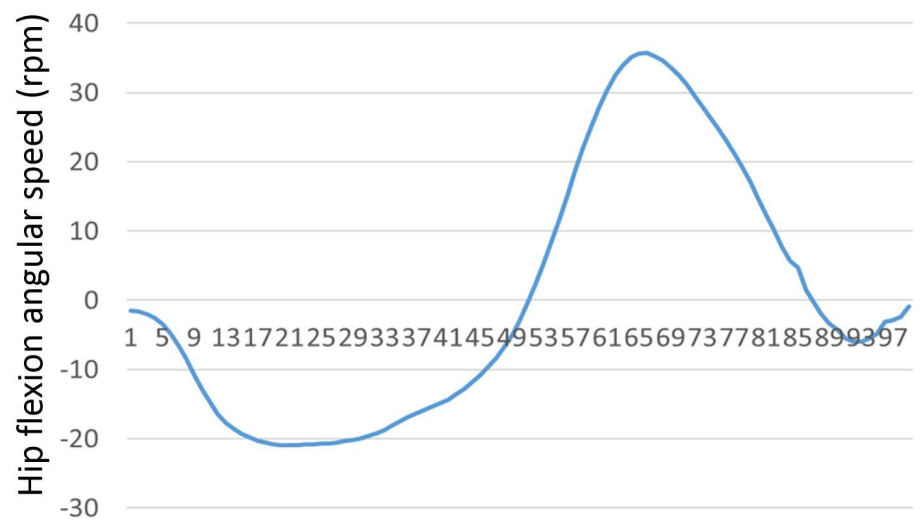

Gait cycle (\%)

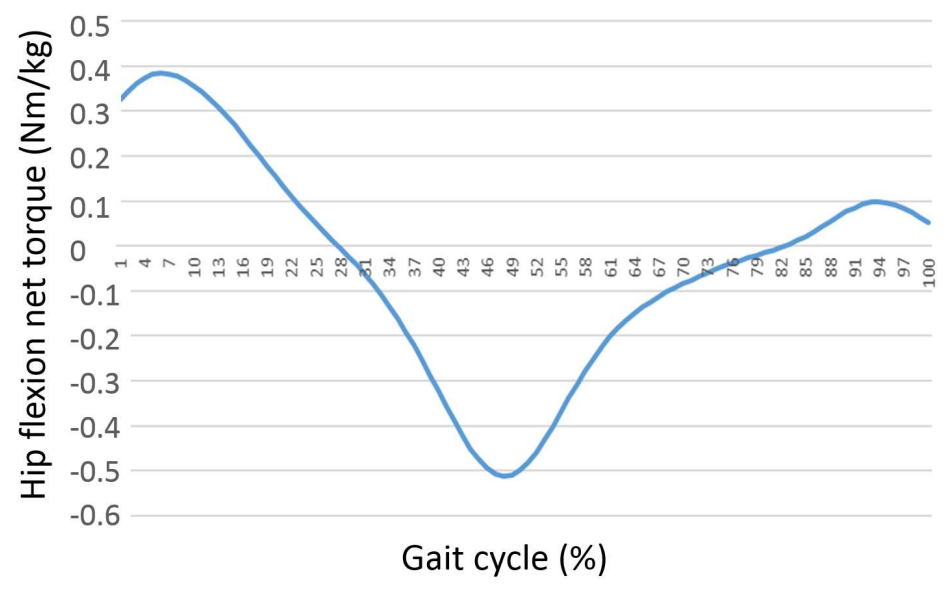

Figure 6.1: Angle, angular velocity and torque at the hip of children aged 6 to 17 years old walking on flat terrain 


\subsubsection{Actuator requirements at the knee}

The maximum torque required at the knee is $0.4 \mathrm{Nm} / \mathrm{kg}$, which is reached in the load response phase (see Figure 6.2). For the total objective weight (user plus exoskeleton) of $51 \mathrm{~kg}$, the required maximum torque is $20.4 \mathrm{Nm}$. The maximum speed is 60rpm as shown in Figure 6.2. It should be noted that during the higher torque demand in the load response phase the speed demand is very low, approx $18 \mathrm{rpm}$ average speed until the maximum torque is reached.

\subsubsection{Actuator requirements at the ankle}

The human ankle is the most powerful joint, especially in the push-off phase, where up to $166 \mathrm{~W}$ of power is required for a child. The required maximum torque is 1.2 $\mathrm{Nm} / \mathrm{kg}$ (see Figure 6.3 which for a weight of $51 \mathrm{~kg}$ results in a torque of $61 \mathrm{Nm}$. The maximum speed is $62 \mathrm{rpm}$.

Therefore, the joint actuation system of an exoskeleton requires high torque peaks, while allowing compliance to the human wearer.

Some solutions to fulfill these requirements have been proposed in literature. Pneumatic actuators with antagonist configuration offer high power output and are able to change stiffness in a biomimetic way. Some rehabilitation devices have been developed with pneumatic actuators [28], but active orthoses must be portable and nowadays pneumatic and hydraulic actuators need power supply that is too large and weighted to be feasible for this application. So an efficient electric actuator is needed to drive the exoskeleton joints. Also it has to be able to change its stiffness fast enough inside a gait cycle. This kind of actuators must be small enough for not to bother the user and have an aesthetic appearance. Also they have to be as light as possible, for not to increase the inertia moment of the limbs and for being easy to handle, put on and off.

Some new electric actuators with variable compliance have been designed, as AMASC designed at Carnegie Mellon University[106], MACCEPA at Vrije Universiteit Brussels[107] and VS-joint and QA-joint mechanisms at the German Aerospace Centre (DLR) [108], and AwAS at the Italian Institute of Technology (IIT)[88], see Table 3.1 but none of them has been implemented in a portable and wearable active orthosis. The reason why these variable stiffness actuators in Table 

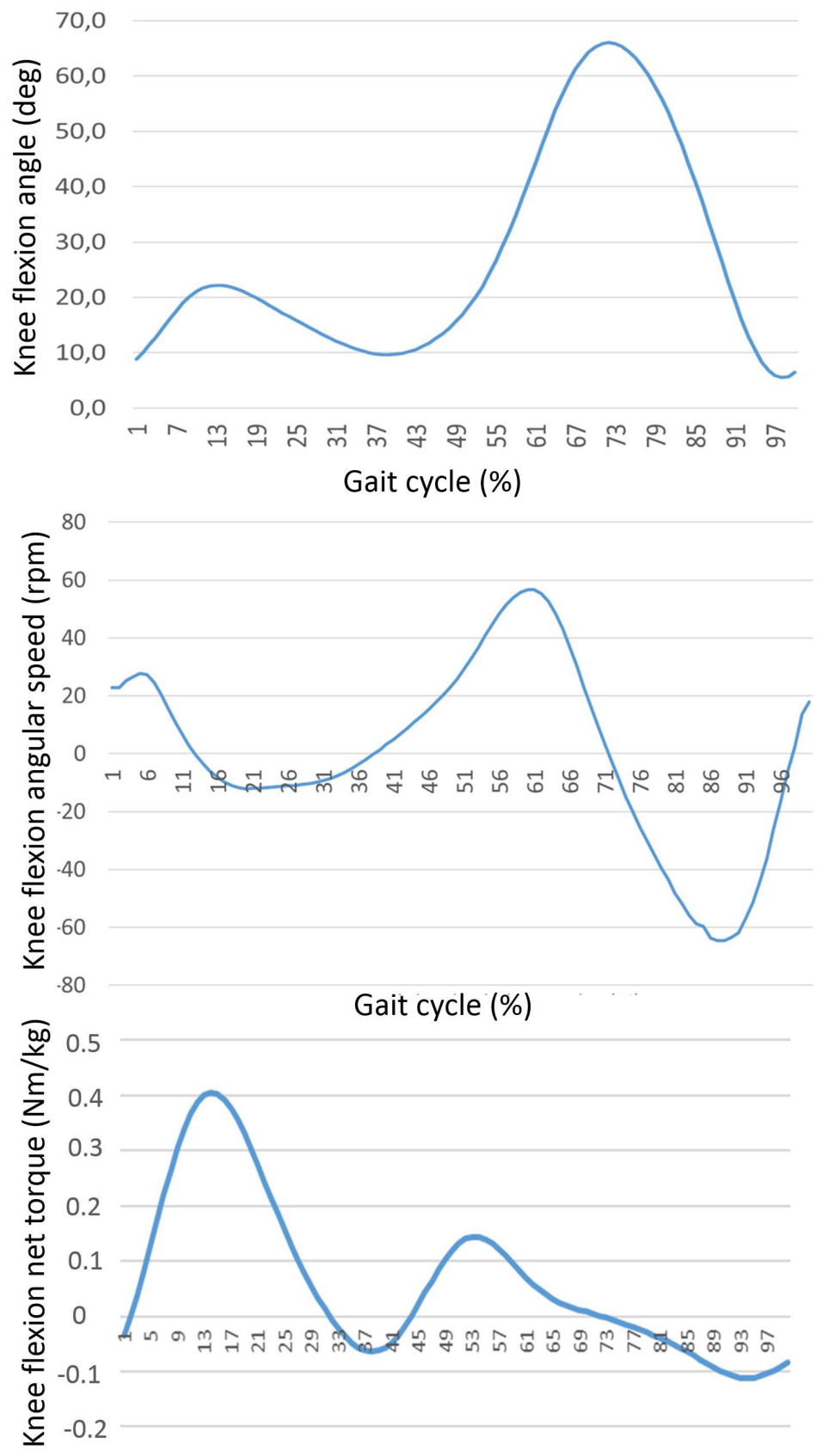

Gait cycle (\%)

Figure 6.2: Angle, angular velocity and torque at the knee walking on flat terrain 


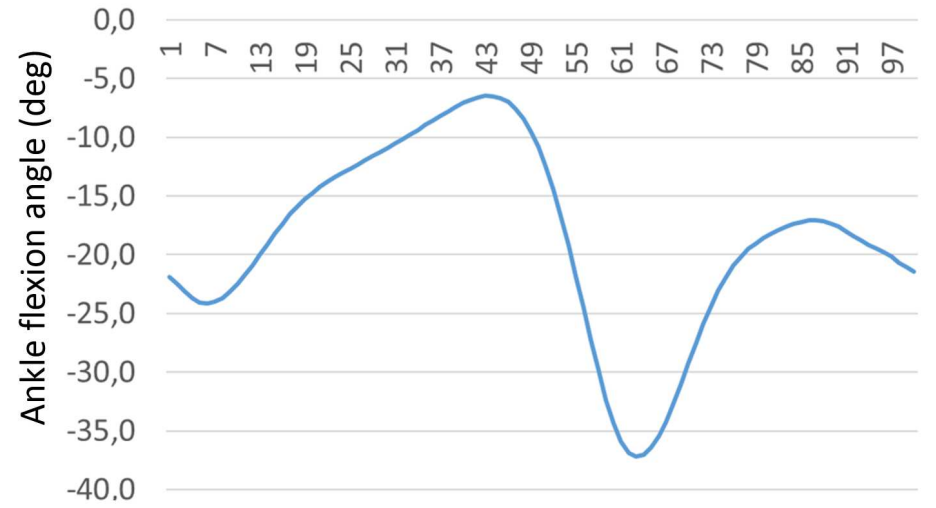

Gait cycle (\%)

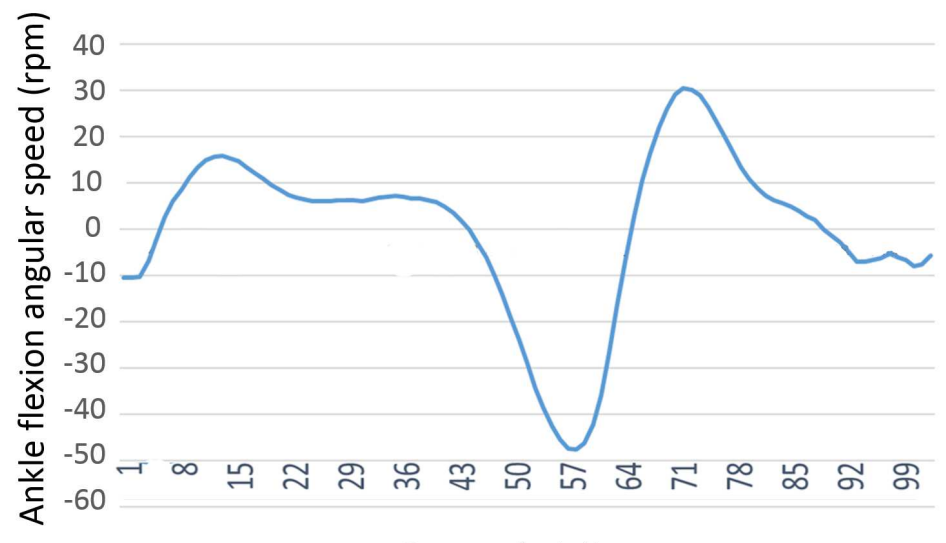

Gait cycle (\%)

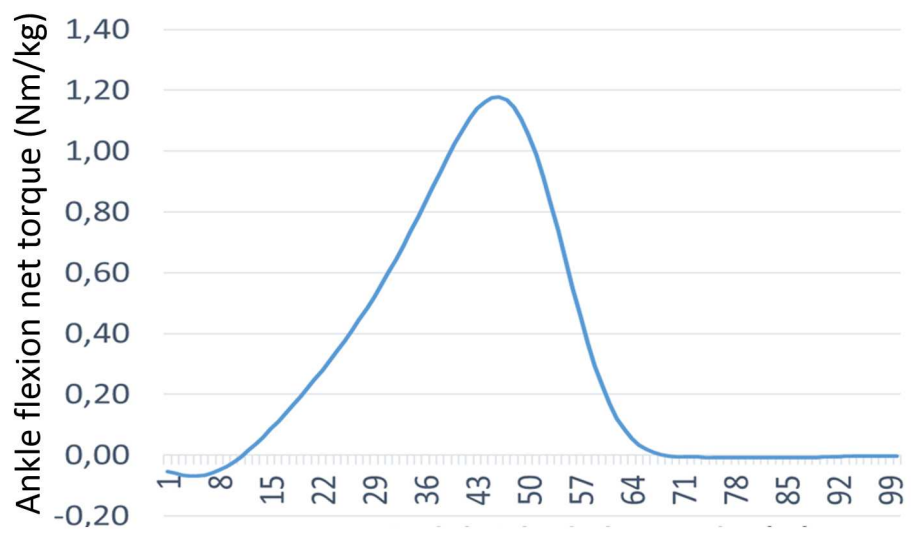

Gait cycle (\%)

Figure 6.3: Angle, angular velocity and torque at the ankle walking on flat terrain 
3.1 are not suitable for use in exoskeletons is listed below, the detail features of these actuator are in Section 3.4:

- Migliore's actuator [83] and VSA II [87] peak torque is not enough.

- MACCEPA [107] actuator has a very slow speed in the stiffness change.

- AMASC [106] has large weight and volume.

- VS-joint and QA-joint mechanisms [108] has large volume.

- Finally, AwAS [88] actuator has too much width.

For this reason, a new prototype that meets these specifications has been designed.

\subsection{Joint stiffness variation in the gait cycle}

Series Elastic Actuators are compliant actuators able to accumulate and release energy. But this thesis holds that in order to take advantage of the potential energy of the springs at different gait speeds and different user weights it is necessary to be able to vary the mechanical stiffness intrinsically in the actuator.

This variation of stiffness at different speeds is observed in human gait. Figures 6.4. 6.5 and 6.6 show torque against position of the hip, knee and ankle joints respectively. The slope of these curves is the stiffness in each phase of the gait. These curves give key information on how the stiffness of each joint is adapted to different speeds of the gait. In this chapter it is shown how the joint stiffness variation during gait is related to an energy consumption strategy. Analyzing the stiffness obtained from the slope of these curves, with respect to each phase of the gait, the following conclutions are obtained:

\subsubsection{Hip stiffness variation}

From the analisys of Figure 6.4, the following conclusions are obtained:

- In the heel strike (HS in Figure 6.4) the stiffness is very high, showing almost a vertical slope, to absorb the impact with the ground. 
- In the loading response phase (LR in Figure 6.4), the stiffness reduces to a low (almost null) value in the mid-stance (MS in Figure 6.4) exploiting the behavior of an actuated inverted pendulum.

- At the terminal stance phase and pre-swing (PS in Figure 6.4) when the hip changes from extension to flexion, the stiffness depends clearly on speed and its behavior is clearly similar to the response of a spring. At slow speed, the stiffness is $0.025 \mathrm{Nm} / \mathrm{kg}^{\circ}$, at normal speed $0.039 \mathrm{Nm} / \mathrm{kg}^{\circ}$, while at fast speed the stiffness reaches a value of $0.075 \mathrm{Nm} / \mathrm{kg}^{\circ}$. This increase of stiffness with velocity is produced to accumulate more potential energy in the tendons to release it as kinetic energy, and thus to achieve a better energy efficiency. Note that in the human gait the stiffness with which the muscletendon system accumulates energy is different from the stiffness when releasing this energy, being always greater the stiffness when this energy is released. This is because the energy is released in a controlled way and the greater the stiffness the better the control over the release of energy.

- When the initial swing is ended, at mid-swing, the hip stiffness remains low (MSw in Figure 6.4). At slow speed the stiffness is nearly zero $0.005 \mathrm{Nm} / \mathrm{kg}^{\circ}$, at normal speed is also very low $0.007 \mathrm{Nm} / \mathrm{kg}^{\circ}$, and it increases at fast speed $0.012 \mathrm{Nm} / \mathrm{kg}^{\circ}$. From the energetic point of view this behavior occurs to take advantage of the intrinsic leg dynamics. When reducing the stiffness to zero the leg behaves like a pendulum without the need of energy supply, except at high speed since in that case the speed of the pendulum is not sufficient for a support in time and it is necessary to provide energy.

\subsubsection{Knee stiffness variation}

The following analysis comes from the knee stiffness behavior shown in Figure 6.5 .

- After heel strike, legs have to stand the body weight in the loading response phase. In Figure 6.5 (LR) it is observed how the stiffness of the knee increases with the speed to absorb and control the kinetic energy in 


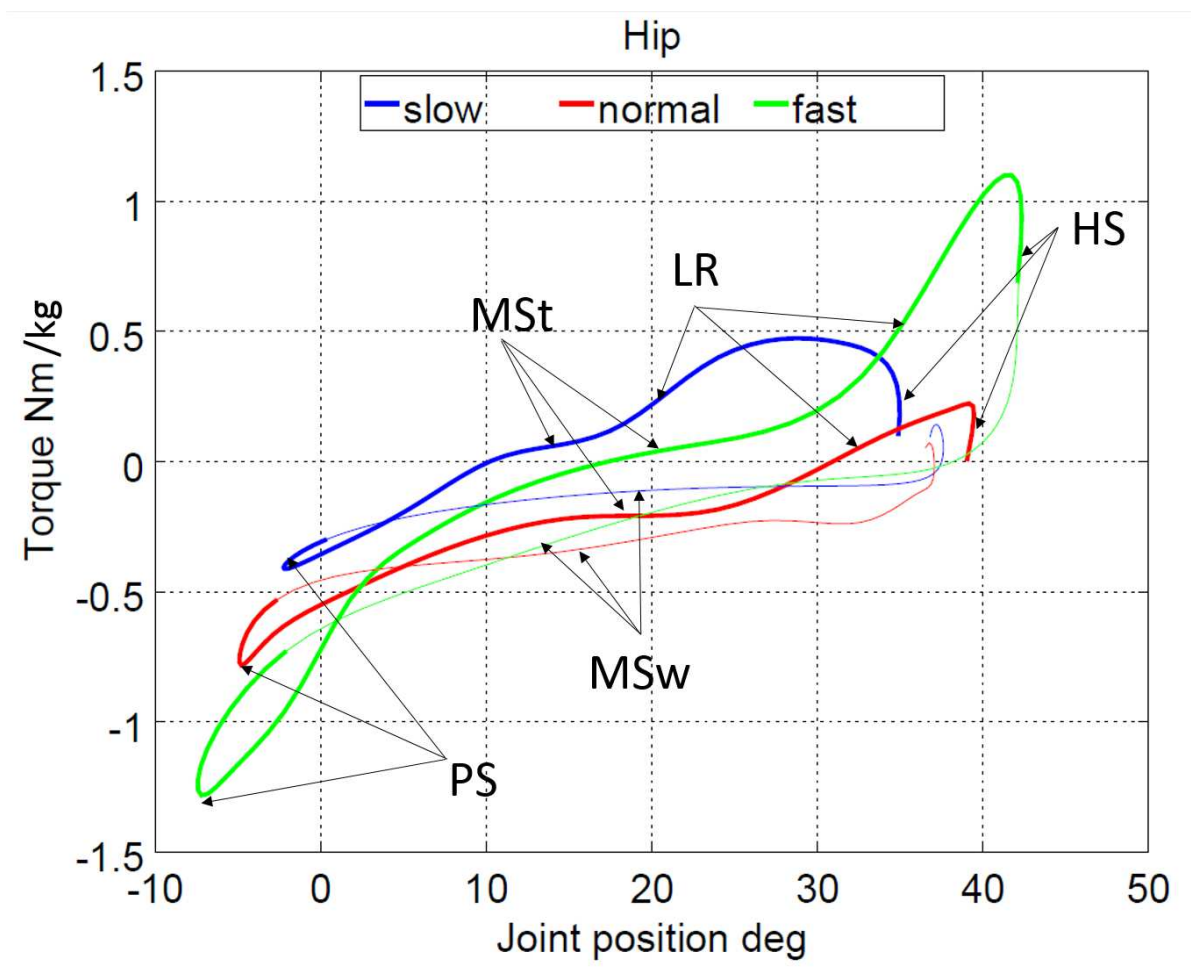

Figure 6.4: Torque vs. position of hip joint walking at slow, normal and fast speeds obtained from [9]. Walking speed normalized to subject's height $\left(\frac{v}{h}\right)$ : slow (blue) for $0.6 \geq \frac{v}{h}<0.8$; medium(red) for $0.8 \geq \frac{v}{h}<1$; fast (green)for $\frac{v}{h} \geq 1$. The support phase is shown in the thickest line. HS - heel strike. LR - Loading response. MSt Mid Stance. PS - Pre Swing. MSw - Mid Swing 
the impact. At slow speed the stiffness is $0.031 \mathrm{Nm} / \mathrm{kg}^{\circ}$, at normal speed is $0.061 \mathrm{Nm} / \mathrm{kg}^{\circ}$, and increases at fast speed $0.69 \mathrm{Nm} / \mathrm{kg}^{\circ}$. Again, it is important to remark that in the human gait the stiffness with which the muscletendon system accumulates energy is different from the stiffness when releasing this energy, being always greater the stiffness when this energy is released. The more the speed increases, the more the stiffness increases, since it is greater the potential energy to control. Thus, in mid-stance phase, at slow speed the stiffness does not increase $\left(0.03 \mathrm{Nm} / \mathrm{kg}^{\circ}\right)$, at normal speed the stiffness increases $0.072 \mathrm{Nm} / \mathrm{kg}^{\circ}$, and at fast speed increases significantly $0.112 \mathrm{Nm} / \mathrm{kg}^{\circ}$ (see Figure 6.5 MSt).

- Once the stance phase is over, in the initial swing the stiffness is nearly null at every speed (see Figure 6.5 ISw phase) and the energy is provided by the inertia of the motion of the hip[117]. This energy that provides the hip is enough for the movement at low speeds, so at low speed the stiffness is zero $\left(0.001 \mathrm{Nm} / \mathrm{kg}^{\circ}\right)$. But at higher speeds although this energy is used, it is necessary to provide torque which is reflected in a non-zero stiffness, at normal speed $\left(0.003 \mathrm{Nm} / \mathrm{kg}^{\circ}\right)$ and fast speed $\left(0.005 \mathrm{Nm} / \mathrm{kg}^{\circ}\right)$.

- In the mid-swing phase the stiffness remains practically null, to take advantage of the potential energy of the shank (see Figure 6.5 MSw phase). Again, this potential energy is enought at low speed so the stiffness is nearly zero $\left(0.001 \mathrm{Nm} / \mathrm{kg}^{\circ}\right)$. But at higher speeds although this potential energy is used, it is necessary to provide torque which is reflected in a non-zero stiffness, at normal speed $\left(0.004 \mathrm{Nm} / \mathrm{kg}^{\circ}\right)$ and fast speed $\left(0.006 \mathrm{Nm} / \mathrm{kg}^{\circ}\right)$.

\subsubsection{Ankle stiffness variation}

A similar analysis is performed along the ankle gait cycle:

- In the heel strike, an intermediate stiffness is maintained in order to absorb the impact along with the other joints. This stiffness increases as the pitch speed increases. See Figure (see Figure 6.6 HS). At slow speed the stiffness is $0.035 \mathrm{Nm} / \mathrm{kg}^{\circ}$, at normal speed is $0.035 \mathrm{Nm} / \mathrm{kg}^{\circ}$, and increases at fast speed $0.053 \mathrm{Nm} / \mathrm{kg}^{\circ}$. 


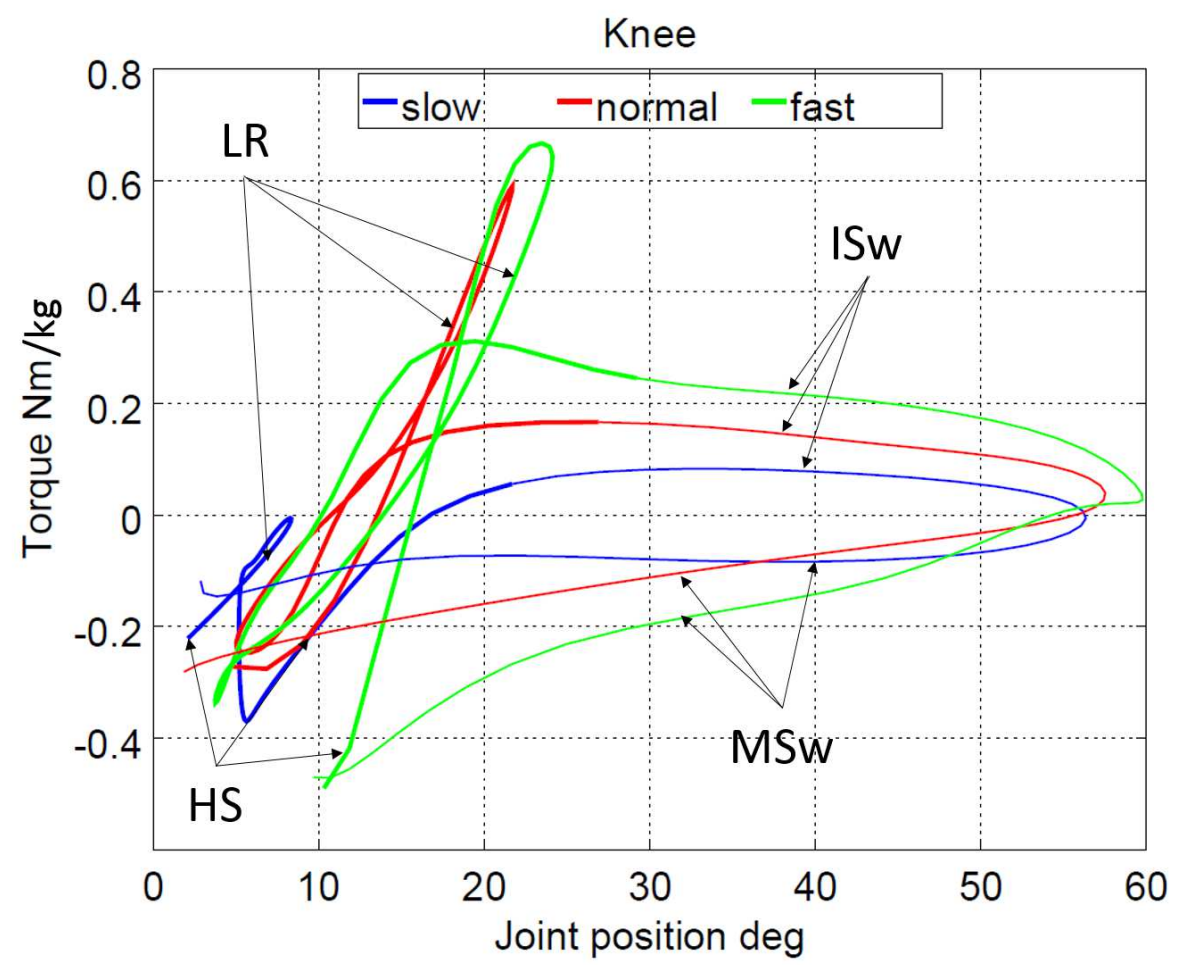

Figure 6.5: Torque vs. position of knee joint walking at slow, normal and fast speeds obtained from [9]. Walking speed normalized to subject's height $\left(\frac{v}{h}\right)$ : slow (blue) for $0.6 \geq \frac{v}{h}<0.8$; medium(red) for $0.8 \geq \frac{v}{h}<1$; fast (green)for $\frac{v}{h} \geq 1$. The support phase is shown in the thickest line. HS - heel strike. LR - Loading response. MSt Mid Stance. ISw - Initial swing. MSw - Mid Swing 
- In the mid-stance phase, the muscle-tendon system accumulates energy, and it does so at a constant stiffness $\left(0.187 \mathrm{Nm} / \mathrm{kg}^{\circ}\right)$ for all velocities.

- In the phase of terminal stance where the accumulated energy is released, it is achieved with lower stiffness than in the previous phase, because it tries to make the most of the energy released. Consequently, it is observed (see Figure 6.6 TSt) as the stiffness even decreases with the speed. At slow speed the stiffness is $0.187 \mathrm{Nm} / \mathrm{kg}^{\circ}$, at normal speed is $0.071 \mathrm{Nm} / \mathrm{kg}^{\circ}$, and decreases at fast speed $0.052 \mathrm{Nm} / \mathrm{kg}^{\circ}$.

So, in the ankle, it is clearly seen how the accumulated energy should be released in a controlled way, modifying the stiffness to achieve the desired torque or speed in each case.

- As the swing phase begins, the stiffness begins to decrease. So that in the mid-swing and terminal swing in the step of dorsiflexion to neutral position $\left(0^{\circ}\right)$ one has zero stiffness in all speeds, since this movement again is realized exploiting the potential energy and the inertia of the rest of joints .

\subsubsection{General impedance considerations}

This behavior of stiffness in each joint is related with the harvesting of energy, in the cases of using the elastics element of the actuator to store and release energy, exploiting passive dynamics during swing or reducing costs at collisions exploiting the intrinsic impedance. As it is shown, at different speeds of walking the stiffness needs to be modulated to control the response of each joint for every purpose. By accumulating energy in the springs in series of an actuator, the kinetic energy is stored into potential energy. Therefore, to be able to damp the impact with the ground at different gait speeds and different weights the stiffness must be changed. If in addition to damping the impact with the ground, it is necessary to use the energy accumulated in the springs of the elastic actuator so that they provide movement without an actuator consuming power in certain phases of the gait, then it is necessary to be able to vary the stiffness. Since depending on the stiffness the speed of recovery of the springs is different, therefore, this stiffness must be intrinsic, not emulated by impedance control algorithms. 


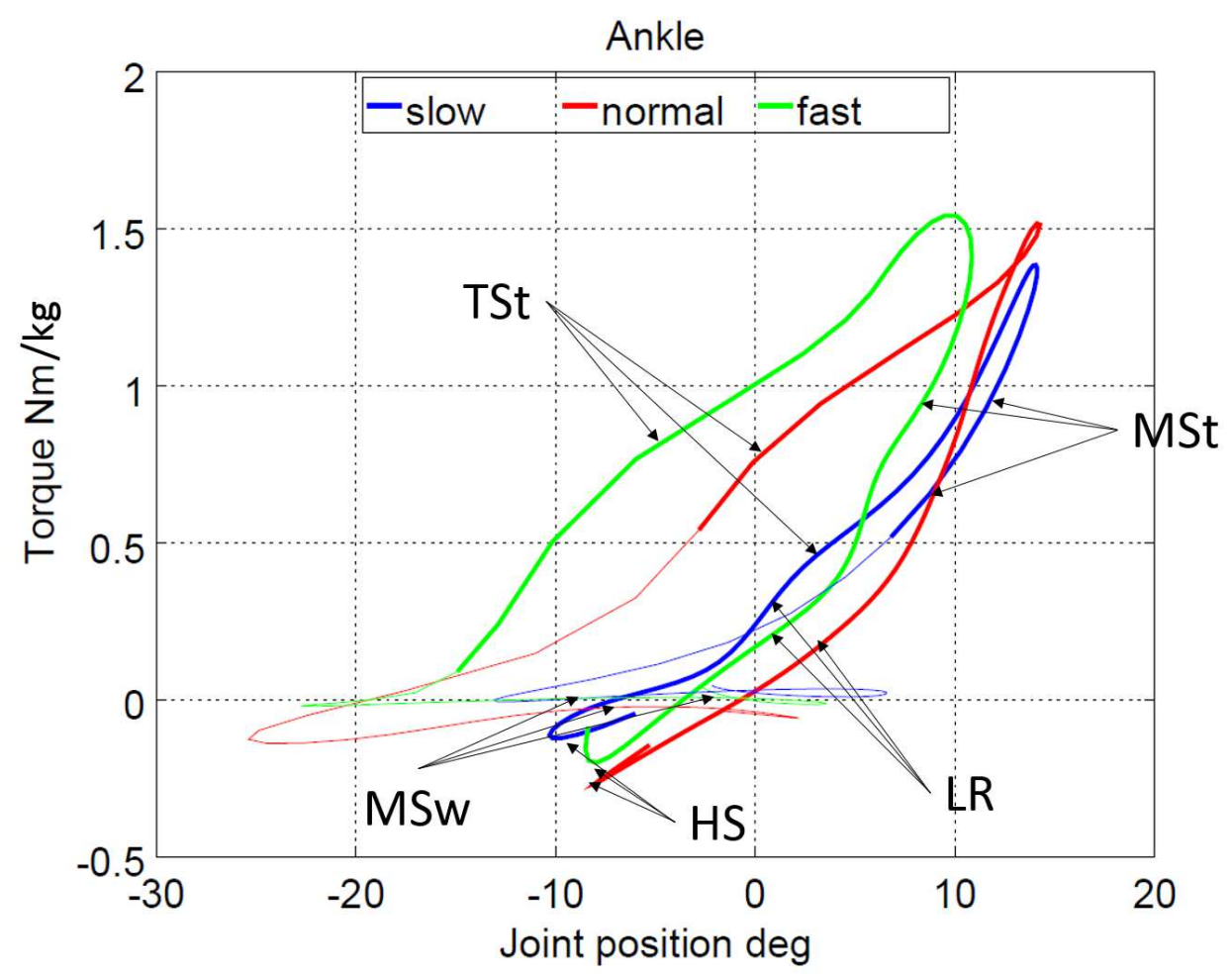

Figure 6.6: Torque vs. position of ankle joint walking at slow, normal and fast speeds obtained from [9]. Walking speed normalized to subject's height $\left(\frac{v}{h}\right)$ : slow (blue) for $0.6 \geq \frac{v}{h}<0.8$;medium(red) for $0.8 \geq \frac{v}{h}<1$; fast (green)for $\frac{v}{h} \geq 1$. The support phase is shown in the thickest line. HS - heel strike. LR - Loading response. MSt Mid Stance. TSt - Terminal Stance. MSw - Mid Swing 
Table 6.1: Motor-gearbox set features

\begin{tabular}{l|l|l|l}
$\begin{array}{l}\text { Motor } \\
\text { Flat Maxon 90W }\end{array}$ & $\begin{array}{l}\text { Gearbox } \\
\text { Harmonic Drive unit }\end{array}$ & $\begin{array}{l}\text { Output speed } \\
2.5 \mathrm{rad} / \mathrm{s}\end{array}$ & $\begin{array}{l}\text { Output torque } \\
70 \mathrm{Nm}\end{array}$ \\
with 160:1 reduction ratio & &
\end{tabular}

\subsection{Adjustable stiffness with embedded sensor (ARES) actuator}

In the first prototype of ATLAS active orthosis, the flexion and extension motion of the hip and the knee joints was driven by electrical brushless flat Maxon motors in combination with Harmonic Drive units. The resulting motor-gearbox set provided enough torque to move the user and showed a large power-to-weight ratio (see Table 6.1 but was too much rigid for a human-machine interaction task.

Based on the above impedance considerations, an actuator prototype that incorporates variable compliance and provides an accurate torque measure has been designed in the frame of the ATLAS project, keeping the large power-to-weight requirements. This actuator has been named ARES: Adjustable stiffness with Embedded Sensor Actuator.

The CAD design of the ARES actuator is shown in Figure 6.7. It consists of two parts, a stiff complex, responsible for generating the main power source of the actuator and a compliant mechanism, responsible for providing the desired stiffness. The compliant mechanism is designed to extend along the leg length to reduce the lateral size of the joint (see Figure 6.7-(b)).

The stiff complex (see Figure 6.7-(c)), consists of an actuator and a gearbox. The actuator is a 90W DC Maxon flat motor, in combination with a Harmonic Drive gearbox of 160:1 radio. This reduction motor combination reaches $2.5 \mathrm{rad} / \mathrm{sec}$ and torque of $70 \mathrm{Nm}$, sufficient to meet the needs of locomotion on flat ground at medium-low speeds. The transmission of torque is made through the bar B1 and the pivot $\mathrm{P} 1$ towards the compliant mechanism.

The compliant mechanism regulates the amount of joint stiffness. A set of elastic elements are placed in a slider system which is connected to a spindle drive for adjusting the slider position along the linear guides of a fixed frame, see Figure 


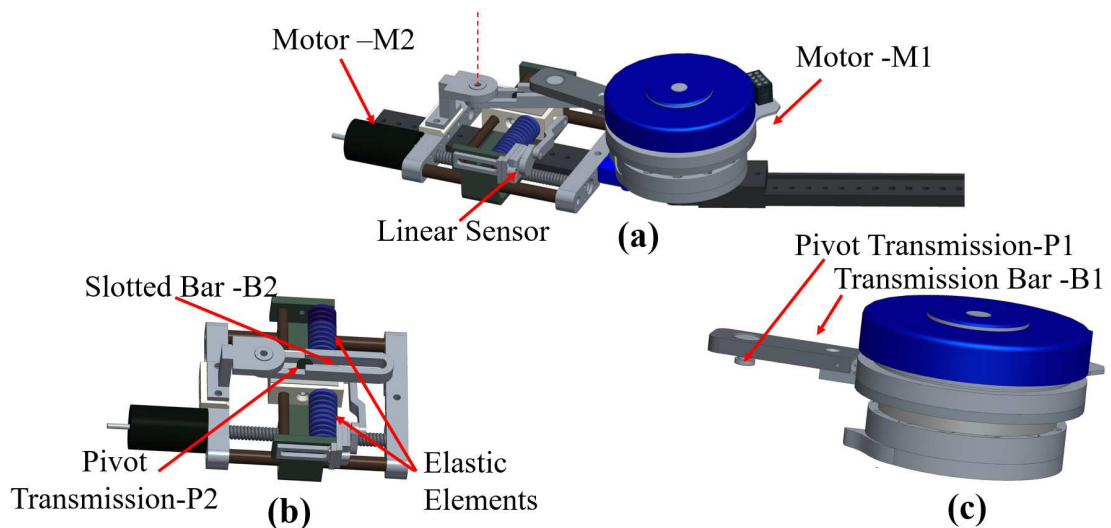

Figure 6.7: ARES Joint Mechanics. a) CAD Drawing of ARES, b) CAD of Compliant Mechanism, and c) CAD of stiff complex.

6.7 (b). The motor that moves the elastic assembly to modify the stiffness is a motor Maxon RE-25 AC of $20 \mathrm{~W}$ (M2). This motor M2 is considerably smaller than the main actuator (M1). This actuator M2 is in charge of modifying the position of the elastic mechanism. The elastic mechanism is connected with a pivot (P2) to a rod (B2) which is connected to the pivot (P1) from where the torque of the stiff complex comes from. By making an analogy of this actuator with the muscletendon model presented in Chapter 2, the stiff complex would be equivalent to the performance of the muscle force generator, the springs have their equivalent in the tendons and the variation of stifness, that the musculo-tendon system achieves by its antagonic configuration, is achieved in the ARES actuator by varying the length of the arm.

\subsection{Working principle of the variable stiffness actu- ator}

This actuator (see Figure 6.11 (c)) is classified based on the work of the VIACTORS consortium [78] as a controllable stiffness ratio actuator in which the principle of operation is as follows. The torque exerted by the main motor (M1) on the joint is transmitted through the pivot P1 to the double bar system B1 and B2. Thus this 
reaction force is transmitted to the transmission pivot $\mathrm{P} 2$ in contact with the elastic elements. The stiffness adjustment is based on the pivot displacement principle in which the actuator position and stiffness control are decoupled achieving more energy efficiency [90]. As it is studied in this paper [90], the fact that the stiffness is regulated by the variation of a level arm, makes the actuator efficient, because the aparent output stiffness can be modified without changing the energy stored internally in the elastic elements.

The force at the transmission point $\mathrm{P} 2$ depends on the torque exerted by the motor M1 and the distance L2 of the elastic elements, see Figure 6.8 .

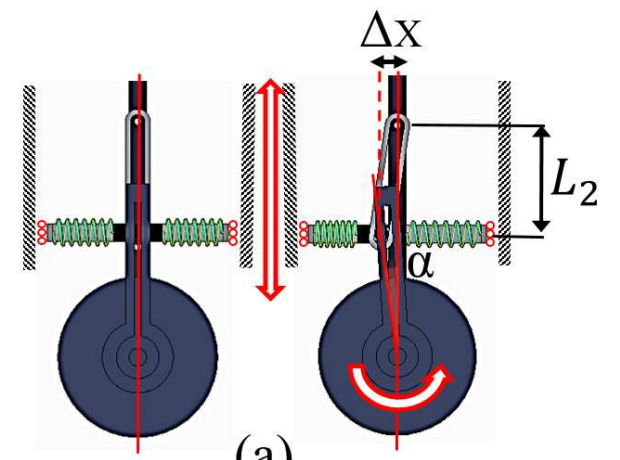

(a)

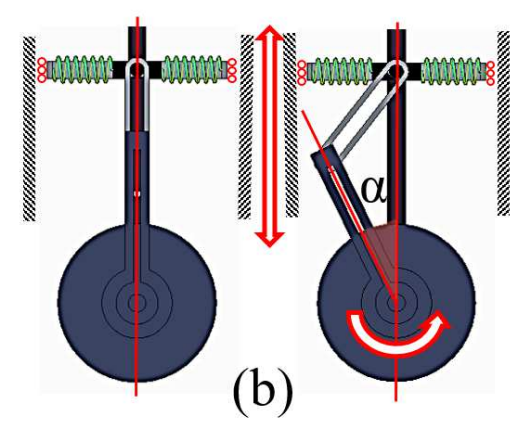

Figure 6.8: ARES Working Principle. a) ARES High Stiffness b) ARES Minimum Stiffness.

The position of the elastic elements relative to the main joint axis, that is the distance L2, is controlled by the motor M2. With a large distance L2, the elastic elements are closer to the joint axis, Figure 6.8-(a), therefore, when the motor M1 exerts torque, it is transmitted to the springs with a short arm which produces a 
significant force in the springs. On the opposite side, for a small L2, the transmited force is lower.

If the distance L2 is zero, Figure 6.8-(b), when the motor M1 exerts a torque the compliance mechanism does not oppose to it and the motor moves freely, with stiffness close to zero. Taking advantage of this feature, the gravity and inertia of the other joints can be exploited to freely move the joint without energy consumption from M1. With intermediate lengths of L2, the stiffness at the output of the actuator can be controlled.

\subsection{Joint speed control}

When motor M2 moves the stiffness varies and it produces a variation in the angle of the joint that is necessary to consider for joint motion control purposes. Based on Figure 6.9 the angle $\alpha$ that the compliant element produces can be computed.

Let the speed of the stiffness variation motor M2 be defined as:

$$
v_{M 2}=\frac{d L_{2}}{d t}
$$

The velocity of the springs deflection $(\Delta X)$ is defined as:

$$
v_{\Delta X}=\frac{\Delta X}{d t}
$$

The angle $\beta$, created between the slotted bar and the line passing through the axis of rotation of the joint and the point of rotation of the slotted bar, is computed from the relationship:

$$
\beta=\operatorname{atan} \frac{\Delta X}{L_{2}}
$$

Thus, the rate of $\beta$ change can be expressed in relation to the velocity of variation of the length of the elastic elements and the rest of known variables, such as:

$$
\frac{d \beta}{d t}=\frac{\Delta X v_{M 2}-L_{2} v_{\Delta X}}{1+\left(\frac{\Delta X}{L_{2}}\right)^{2}}
$$

Applying the sine theorem, $\alpha$ is related to $\beta$ by: 


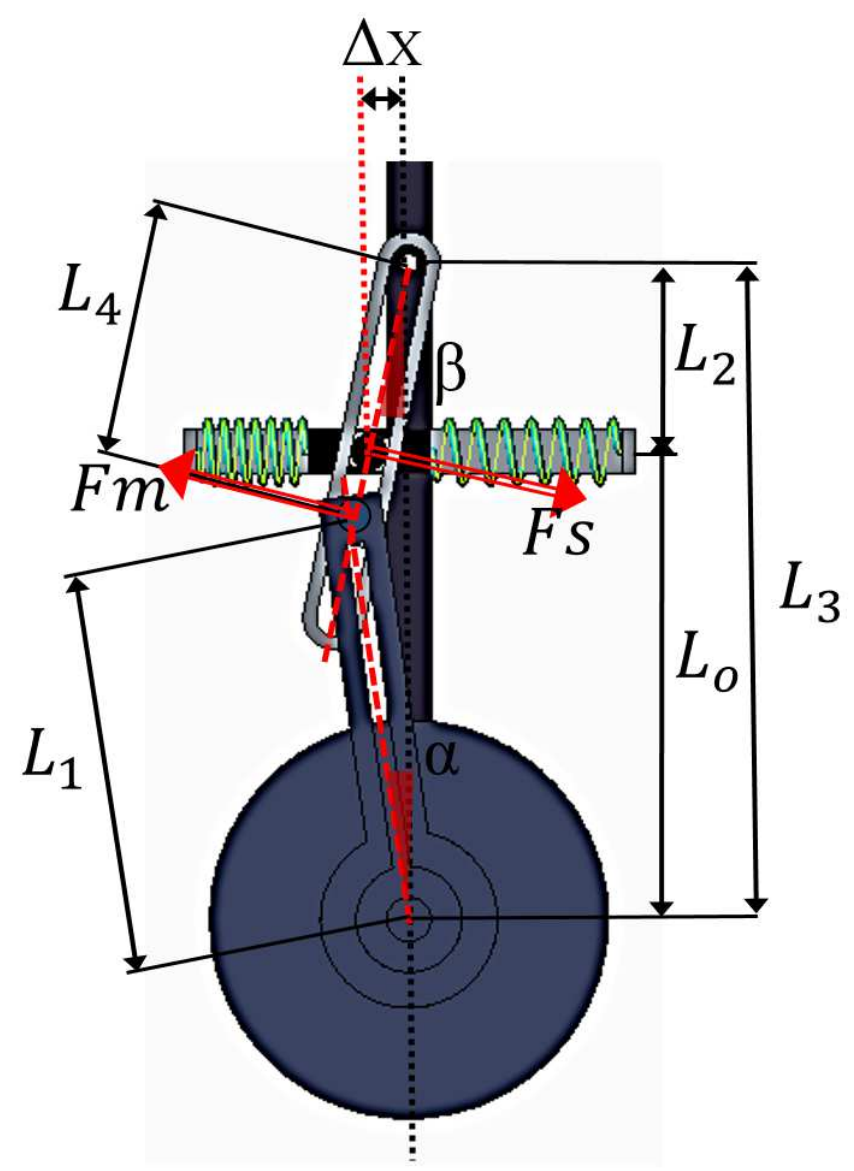

Figure 6.9: Diagram for the calculation of velocities and torques. 


$$
\alpha=\operatorname{asin}\left(\frac{L_{4} \sin \beta}{L_{1}}\right)
$$

Being $L_{4}$ the distance between the coupling point and the pivot bar:

$$
\left(L_{4}\right)^{2}-\left(2 L_{3} \cos (\beta)\right) L_{4}+\left(\left(L_{3}\right)^{2}-\left(L_{1}\right)^{2}\right)=0
$$

where $L_{1}$ and $L_{3}$ are constants.

Deriving from equation 6.5 , the angular speed of the joint $\frac{d \alpha}{d t}$ related to the rate of stiffness variation $v_{M 2}$ and the speed of spring compression $v_{\Delta X}$ can be obtained by the equation 6.4 and the equations:

$$
\frac{d \alpha}{d t}=\frac{\left(L_{4} \sin \beta\right)^{\prime}}{L_{1} \sqrt{1-\frac{L_{4} \sin \beta}{L_{1}}}}
$$

where

$$
\left(L_{4} \sin \beta\right)^{\prime}=\frac{d L_{4} \sin \beta}{d t}
$$

and

$$
\frac{d L_{4} \sin \beta}{d t}=\frac{d \beta}{d t} *\left(L_{3} \cos (2 \beta)+\cos (\beta) L_{3} \sqrt{\cos ^{2} \beta-L_{1}^{2}}-\frac{L_{3} \sin (2 \beta) \sin (\beta)}{2 L_{3} \sqrt{\cos ^{2} \beta-L_{1}^{2}}}\right)
$$

When changing the stiffness, this joint angle variation must be taken into account. Moreover, this joint angle variation can be harnessed to save energy; while modifying the stiffness it can be used for the motor M2 to move the joint reducing the energy consumption of M1. This power-saving application will be shown in experimental validation in Chapter 7.

\subsection{Force measurement embedded in the ARES actu- ator}

The elastic elements of the ARES actuator, besides providing compliance to the joint, are used to acquire the joint torque.

The linear displacement $\Delta X$ of the elastic elements is measured by means of a magnetic sensor. The translation movement of the springs is translated into a rotational movement with a crank-crank mechanism. This angle of rotation is measured 
with a magnetic encoder and from this angle, linear displacement $\Delta X$ is calculated.This deflection together with the mechanical design of the actuator allows to calculate the torque made by the system.

Given the diagram of the joint of Figure 6.9, the joint torque can be written as:

$$
\tau=\frac{F_{m} L_{1}}{\cos \alpha}
$$

The force $F_{s}$, transmitted through the slotted bar will be orthogonal to -B2 and the angle between the springs axis and the force equals $\beta$. Hence, given the springs elastic constant $K$ and the compression $\Delta X$, for identical elastic elements:

$$
F_{s}=\frac{2 \Delta X K}{\cos \beta}
$$

Since the transmission is made by the same bar, the torque by $F_{s}$ equals the torque by $F_{m}$, therefore:

$$
L_{4} F_{m}=L_{2} F_{s}
$$

Combining equations 6.10, 6.11 and 6.12 the joint torque can be computed with the known variables, from the compression of the springs:

$$
\tau=\frac{L_{2} L_{1} 2 \Delta X K}{L_{4} \cos (\alpha) \cos (\beta)}
$$

The system can achieve a wide range of stiffness. With $L_{2}$ set to zero, the stiffness of the joint will be zero and can move freely without resistance. At this point the joint has a range of $20 \mathrm{deg}$, which can be used to emulate the behavior of the knee in the phase where the underactuated strategy is applied.

\subsection{Simulation-based performance evaluation}

In order to evaluate the ARES actuator concept performance in terms of both force sensing and stiffness variation in a quantitative way, simulations have been performed with the help of the CAD software CREO Parametric [118]. For this purpose a simplified model has been used which contains the main elements and physical properties of the ARES actuator. 
Table 6.2: Variation of stiffness with actuator arm length

$\begin{array}{ll}\text { Length } L_{0}(\mathrm{~cm}) & \text { Stiffness }(\mathrm{Nm} / \mathrm{deg}) \\ 130 & 0 \\ 108 & 1,33 \\ 98 & 3,07 \\ 80 & 6,31 \\ 70 & 10\end{array}$

Different torque values were commanded while the spring compression values and the joint deflection were provided by the simulation, while the arm length $L_{0}$ was varied. These parameters are those to be measured in the actual model and the arm length can be varied with the motor M2.

Figure 6.10 shows the results obtained in this simulation. Figure 6.10 (a) shows the spring compression related with the torque exerted, while the Figure 6.10(b) shows the joint deflexion related with the torque exerted at different values of $L_{0}$. Therefore, the slope of these lines, in the Figure 6.10(b), for each value of $L_{0}$ represents the stiffness value. Table 6.2 shows the stiffness values for the set of values of $L_{0}$.

So, from the values of the Table 6.2 with a linear regression, the value of stiffness is obtained from the distance $L_{0}$ :

$$
K_{\text {eqact }}=-0,163 L_{0}+20
$$

The strategies of stiffness variation in the human gait described in Sections 6.2.1 6.2.2 and 6.2.3 will be implemented in ARES actuator to validate the hypothesis of energy reduction through strategies of stiffness adaptation. The analysis will be based on a prototype of ARES and a series of experimental tests, presented in Chapter 7.

\subsection{ARES prototype}

An instantiation of ARES was manufactured with the requirements of the joints of the active orthosis ATLAS. It was mainly made of aluminum except the force trans- 


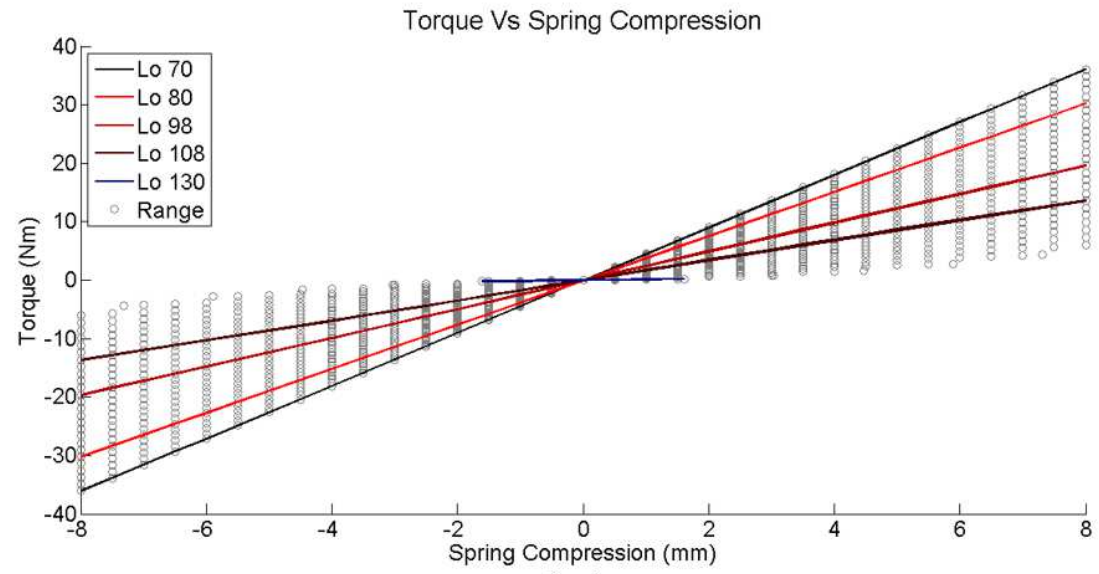

(a)

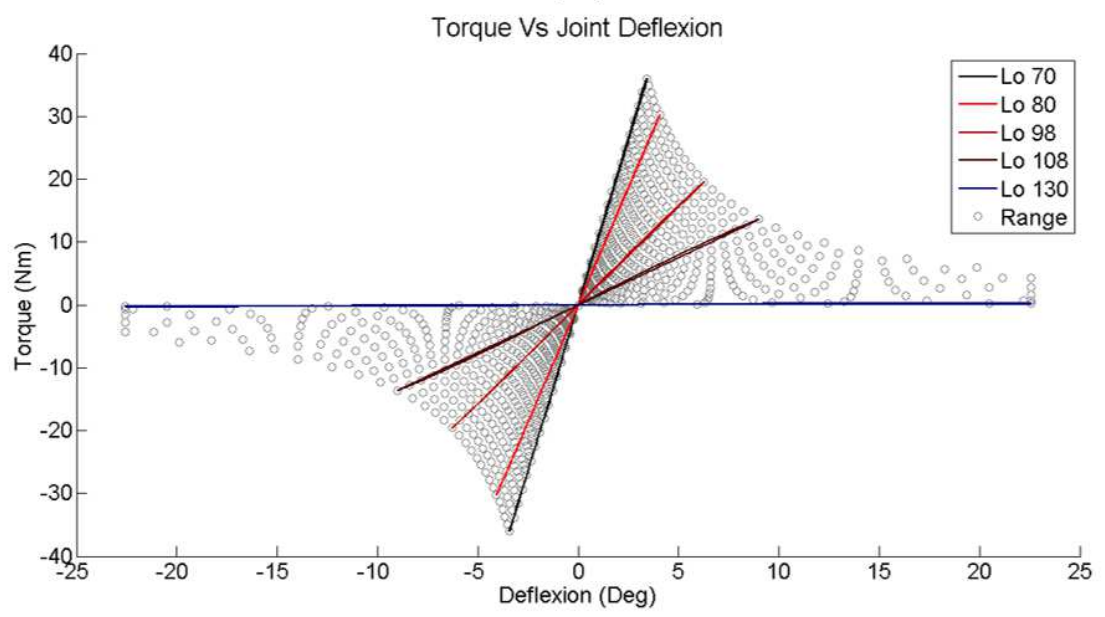

(b)

Figure 6.10: ARES Range of Stiffness. a) Torque Range for Different Positions of Lo b) Stiffness Range for Different Positions of Lo.. 


\subsection{ARES prototype}

mission bar which was made of steel to withstand the force of the pivot. Some other components like the encoder housing of force measurement were manufactured in $3 \mathrm{D}$ printing plastic.

Figure 6.11 (a,b and c) shows the prototype ARES, where the motor M1 for joint motion and M2 for the variation of stiffness are observed. Also the components of the elastic mechanism: set of springs, bars and pivots are highlighted. Table 6.3 summarizes the main mechanical properties of the prototype.

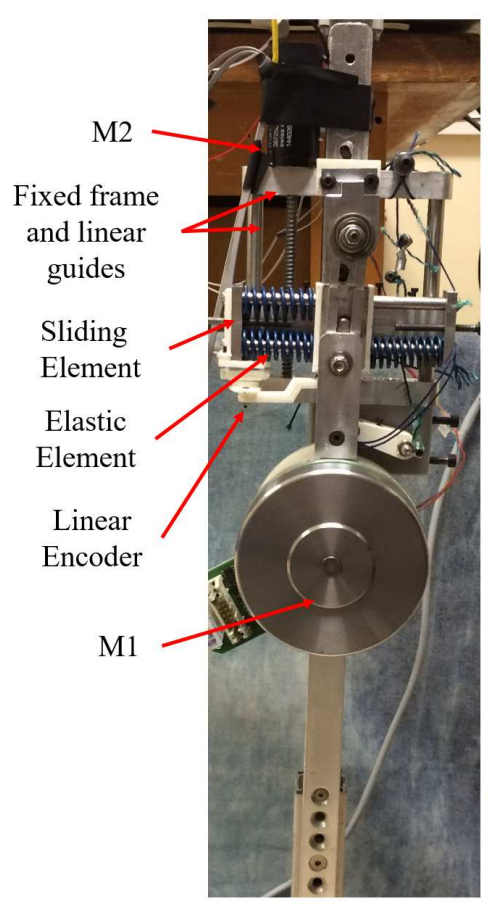

(a)
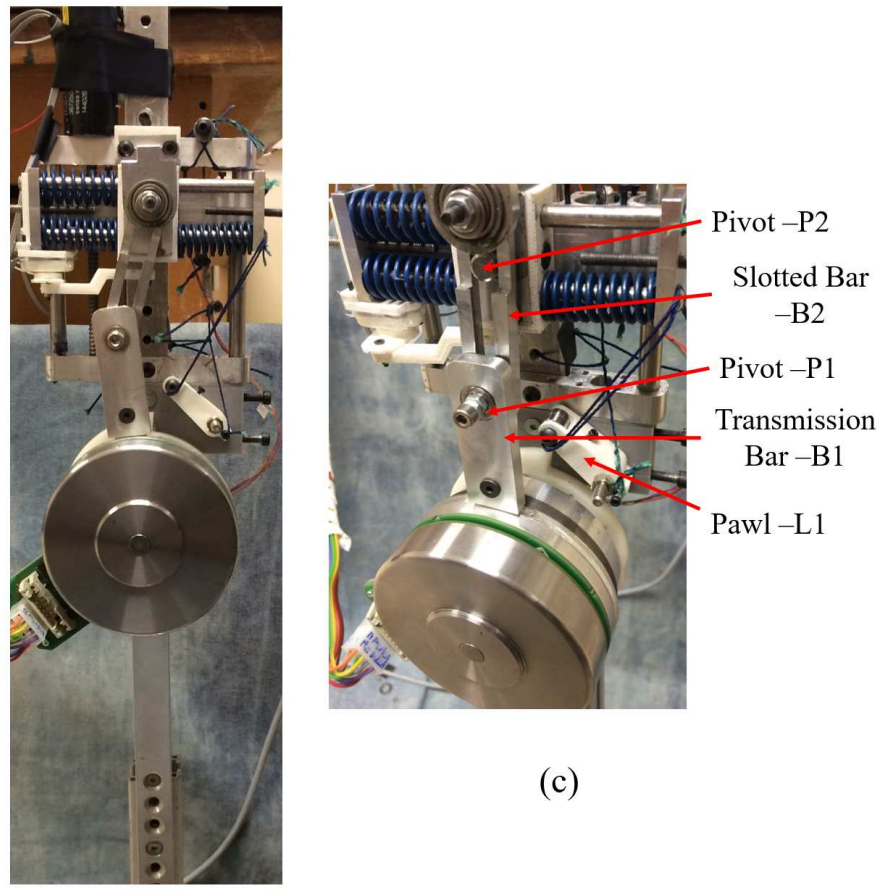

(c)

Figure 6.11: ARES Prototype.a) ARES Prototype-Front View. Maximum stiffness. b) ARES Prototype-Front View. Stiffness Near Zero. c) ARES Prototype - Coupling Elements Highlighed

The prototype will be the basis for the energy-efficient locomotion proposed in this thesis. Next Chapter will present experimental results and performance analysis. 
Table 6.3: ARES Prototype Mechanical Properties

$\begin{array}{ll} & \text { ARES Properties } \\ \text { Peak Torque } & \text { Up to 76 Nm } \\ \text { Max Deflection } & \pm 23^{\circ} \\ \text { Range of Stiffness } & 0-15 \mathrm{Nm} / \mathrm{deg} \\ \text { Stiffness Adjusting time } & 0.25 \mathrm{sec} \\ \text { Weight } & 1.3 \mathrm{Kg} \\ \text { Length } & 260 \mathrm{~mm} \\ \text { Width } & 80 \mathrm{~mm} \\ \text { Power } & 90 \mathrm{~W} \\ \text { Speed } & 20 \mathrm{rpm}\end{array}$

\subsection{Conclusions}

This chapter presents the performance requirements for the movement of the joints for a lower limb exoskeleton for children.

A detailed study is developed about the characteristics of stiffness variation at the hip, knee and ankle flexion joints in the gait cycle. It is concluded that the behavior of the stiffness in each joint is closely related with the energy consumption. Thus, the main characteristics and operating principle of the ARES actuator are presented, on which the stiffness variation strategies that have been studied in this chapter will be implemented. 
Energy and persistence conquer all

things.

Benjamin Franklin

CHAPTER

\section{Energy-efficient control of \\ locomotion}

\subsection{Introduction}

In the previous chapters, a series of strategies to reduce energy consumption were deduced and presented, both from human biomechanics (Chapters 2 and 6) and from a robot in simulation (Chapter 4). The strategies proposed make necessary devices and control schemes capable of dynamically modifying the stiffness of the joints while controlling their trajectory. In this chapter, the results of the implementation of stiffness variation to reduce energy consumption are validated and implemented in the actuators of the ATLAS exoskeleton.

\subsection{Hibrid control approach}

As concluded along this thesis, the control scheme to achieve energy efficient strategies have to exploit natural dynamics, provide position/trajectory tracking in all gait phases, and stiffness regulation. All these strategies must be sincronized to achieve an energy efficient locomotion. Using ARES actuator both the position and the stiffness are controlled. Figure 7.1 presents the general gait control strategy 


\section{ENERGY-EFFICIENT CONTROL OF LOCOMOTION}

implemented in the ATLAS active orthosis to emulate the human gait in order to reduce energy consumption. It consists in a hybrid control strategy, in which three main algorithms are adequately syncronized to maintain the lowest energy consumption as possible.

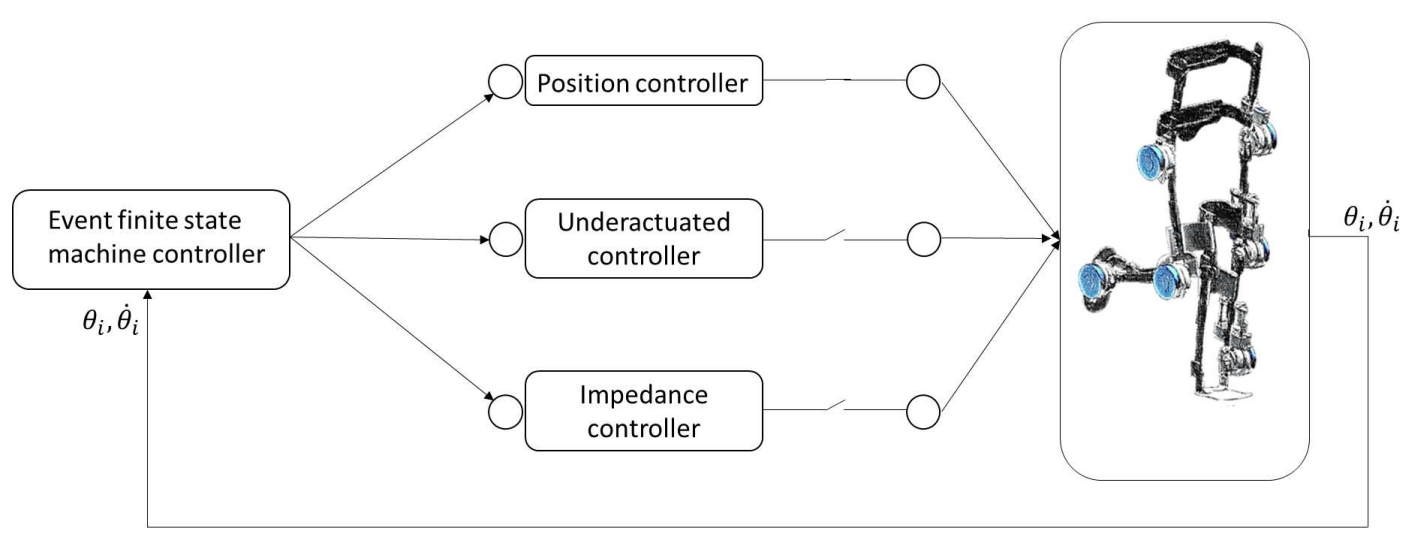

Figure 7.1: Hybrid control scheme

The position controller is selected for those phases of the gait where the largest stiffness is required to maintain position rigid enough to be able to absorb impacts. (see Figure 7.2). It is important to note that the position control is not totally rigid, since according to the design of the actuator presented in Chapter 6 its maximum stiffness is $10 \mathrm{Nm} / \mathrm{deg}$. Motor M1 controller follows the position defined in the trajectory generator using a PID controller. In Section A6 of Appendix A, the control scheme implemented in the ATLAS exoskeleton is detailed. The gait phases in which this position control is applied are the support phases for all joints. This control is also applied in the gait phases of the end-stance to prepare the heel-strike and perform controlled support with the ground.

The underactuated controller is selected for those phases of the gait where the potential and kinetic energy calculated from equation (A.17) in the Appendix A is enough to be exploited, by establishing zero stiffness, the desired trajectory is followed with no energy consumption (see Figure 7.3), for which a null stiffness is established as a reference in the M2 motor. According to Table 6.2, this null stiffness corresponds with a length of $L_{0}$ of $130 \mathrm{~cm}$. The control on the M2 motor 


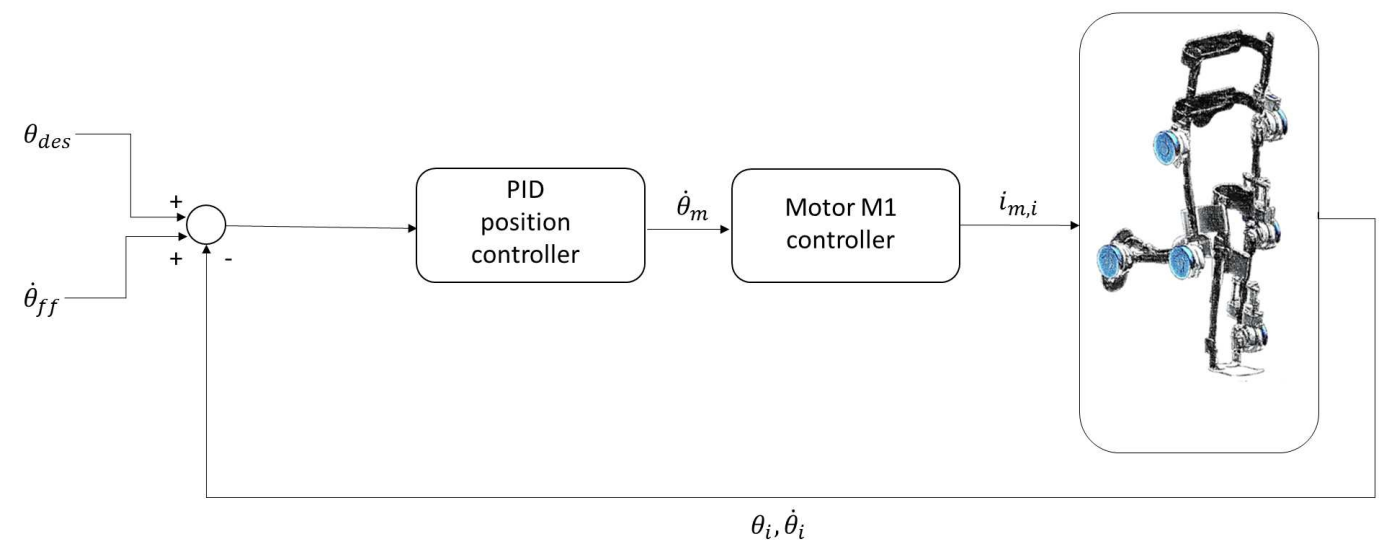

Figure 7.2: Position control scheme

of stiffness variation is a PID control with feedback of the position quadrature encoder on which the measure of the length $L_{0}$ has been adjusted.

The gait phases in which this underactuated control is applied are those of the mid-swing in both the knee and the hip joints to exploit the potential and inertial energy that are accumulated in these phases.

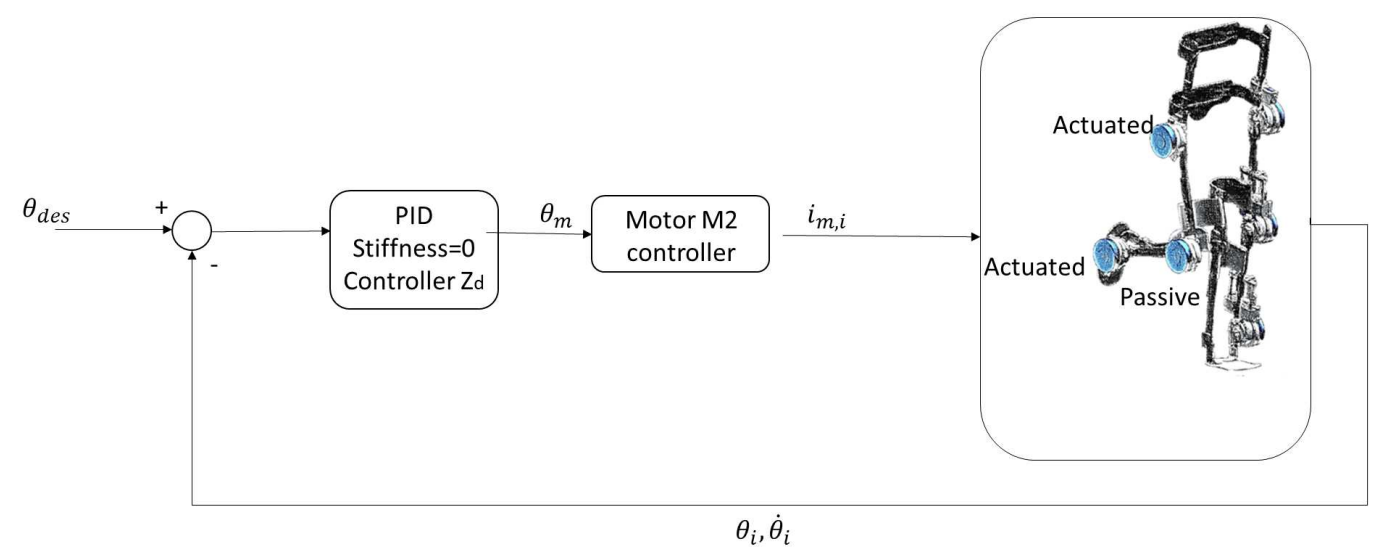

Figure 7.3: Subactuated control scheme

The impedance controller is selected for those phases of gait where energy can be stored in an elastic element, where the needed stiffness is established depending on the phase and the velocity of the gait and the weight of the user (see Figure 7.4). 


\section{ENERGY-EFFICIENT CONTROL OF LOCOMOTION}

The gait phase where the application of this impedance control is more outstanding is in the push-off phase at the ankle, where the energy accumulated during the support phase is released.

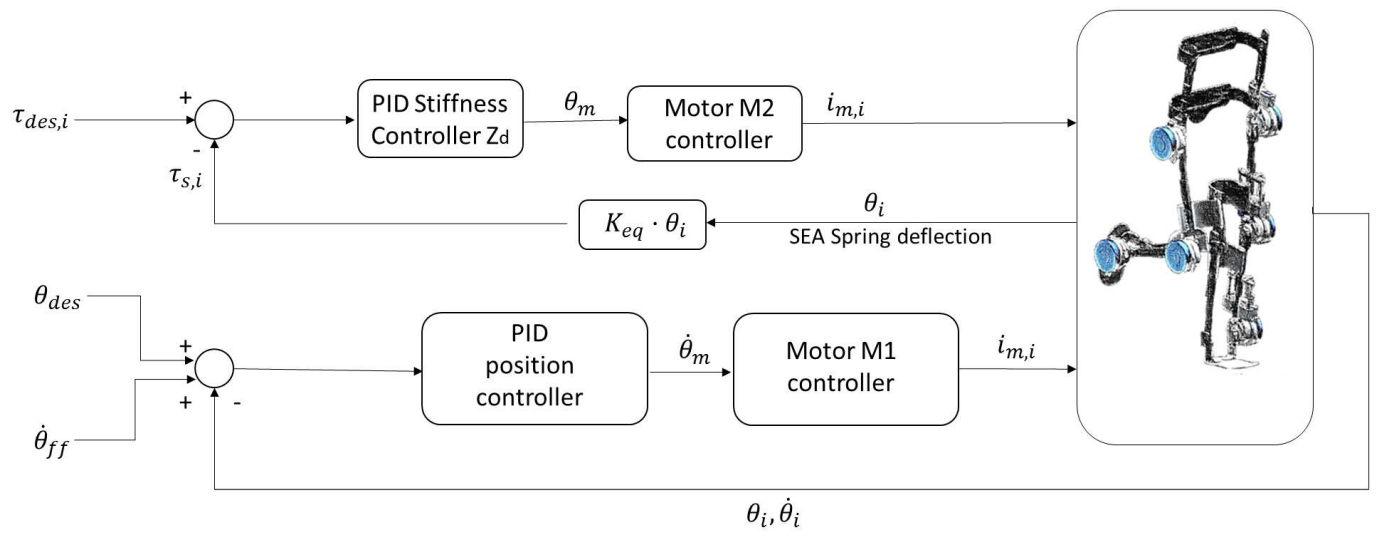

Figure 7.4: Impedance control scheme

\subsection{Joint-level control system}

In order to develop the hybrid control scheme shown above, the position and stiffness of each joint has been independently controlled and a state machine based on the behavior of each joint and the evolution of its kinetic and potential energy has been implemented for each joint .

As it is shown in the Chapter 6, given the design of the ARES actuator, changing the stiffness (with the M2 motor) produces a variation in the position of the output angle. Therefore, a joint-level control approach is designed as shown in Figure 7.5. In this control approach, $\theta_{d}$ is the trajectory commanded to the joint, obtained from the inverse kinematics of the foot trajectory. This trajectory generation is defined in Section A5 of the Appendix A. In order to reach the desired angular position, a PID controller in motor M1 is used. To improve the control a pre-feed of the desired speed $\dot{\theta}_{M 1 r e a l}$ is used which is known. Also, it is taken into account that the movement of the pivot of stiffness variation affects to the movement the angular position in the output of M1, in an angle $\theta_{M 2}$ (see Equation 6.8). So the control 
signal velocity sent to the PID controller to the motor M1 $v_{\text {sent }}$ is (see control diagram in Figure 7.5):

$$
v_{\text {sent }}=K_{p 1}\left(\theta_{M 1 \text { real }}+\theta_{M 2}-\theta_{\text {des }}\right)+K_{d 1}\left(\dot{\theta}_{M 1 r e a l}-\dot{\theta}_{\text {des }}\right)
$$

where $\theta_{M 1 \text { real }}$ and $\dot{\theta}_{M 1 \text { real }}$ are the actual position and speed of the motor M1 respectively, $\theta_{d e s}$ and $\dot{\theta}_{\text {des }}$ are the desired position and speed respectively, and $\theta_{M 2}$ is the angle modification of the angle M1 produced by the motion of M2.

In the control of the motor $\mathrm{M} 2, K_{\text {des }}$ is the is the desired stiffness obtained from the state machine established in the hybrid strategy at each joint. From Equation 6.13, each level of stiffness is related with a desired position $d_{d M 2}$ of the sliding pivot in the arm, which is controlled by the motor M2. Therefore, in order to reach the desired stiffness level, a PID control is performed, with the feedback of the position of the sliding pivot $d_{M 2 \text { real }}$. (see control diagram in Figure 7.5) The control signal position sent to the PID controller to the motor M2 $p_{\text {sent }}$ is:

$$
p_{\text {sent }}=K_{p 2}\left(d_{M 2 r e a l}-d_{d M 2}\right)
$$

where $d_{M 2 \text { real }}$ and $d_{d M 2}$ are the actual and the desired position of the motor M2, respectively.

\subsubsection{Stiffness control at the hip}

An ARES actuator prototype has been incorporated in the hip joint of the ATLAS active orthosis. From the data obtained from the biomechanics of the human gait (Section 6.2.1), a hybrid control strategy has been implemented, represented in the state machine (see Figure 7.6) based on the following behavior in each phase of the gait:

- State 1. Throughout the support phase the maximum stiffness allowed by the actuator of $10 \mathrm{Nm} / \mathrm{deg}$ has been maintained. This emulates the hip stiffness pattern of human gait except for a very low stiffness at mid stance, to exploit the behavior of the inverted pendulum, but this strategy can not been implemented in the ARES actuator because is not able to recover from null to maximum stiffness in just an instant with the weight load of the user, since 


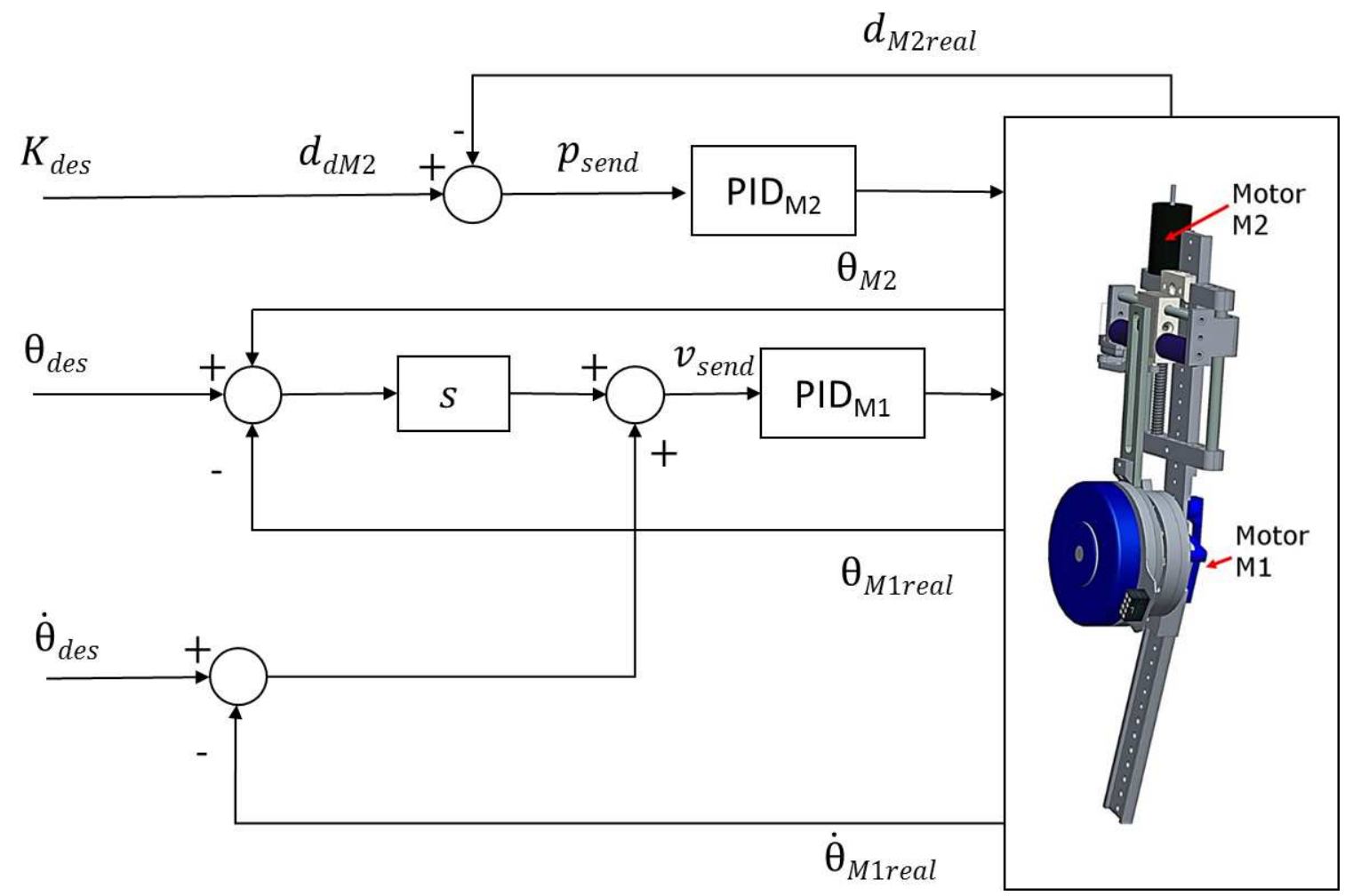

Figure 7.5: ARES joint-level control diagram. This diagram describes the control scheme of the two motors, the movement of the motor M1 that moves the joint and motor M2 of the stiffness variation. $\theta_{M 1 \text { real }}$ and $\dot{\theta}_{M 1 \text { real }}$ are the actual position and speed of the motor M1 respectively, $\theta_{\text {des }}$ and $\dot{\theta}_{\text {des }}$ are the desired position and speed respectively, and $\theta_{M 2}$ is the angle modification of the angle M1 produced by the motion of M2. $d_{M 2 \text { real }}$ and $d_{d M 2}$ are the actual and the desired position of the motor M2, respectively. The desired position is obtained from the desired stiffness Kdes. The velocity sent to the PID controller to the motor M1 is $v_{\text {sent }}$. The position sent to the PID controller to the motor M2 is $p_{\text {sent }}$ 
the motor M2 has not been dimensioned to develop this power. However, neglecting this effect does not make a significant difference with human gait.

- State 2. In the mid-swing phase, a zero stiffness is established, taking advantage of the direct pendulum behavior of the leg by exploiting leg inertia.

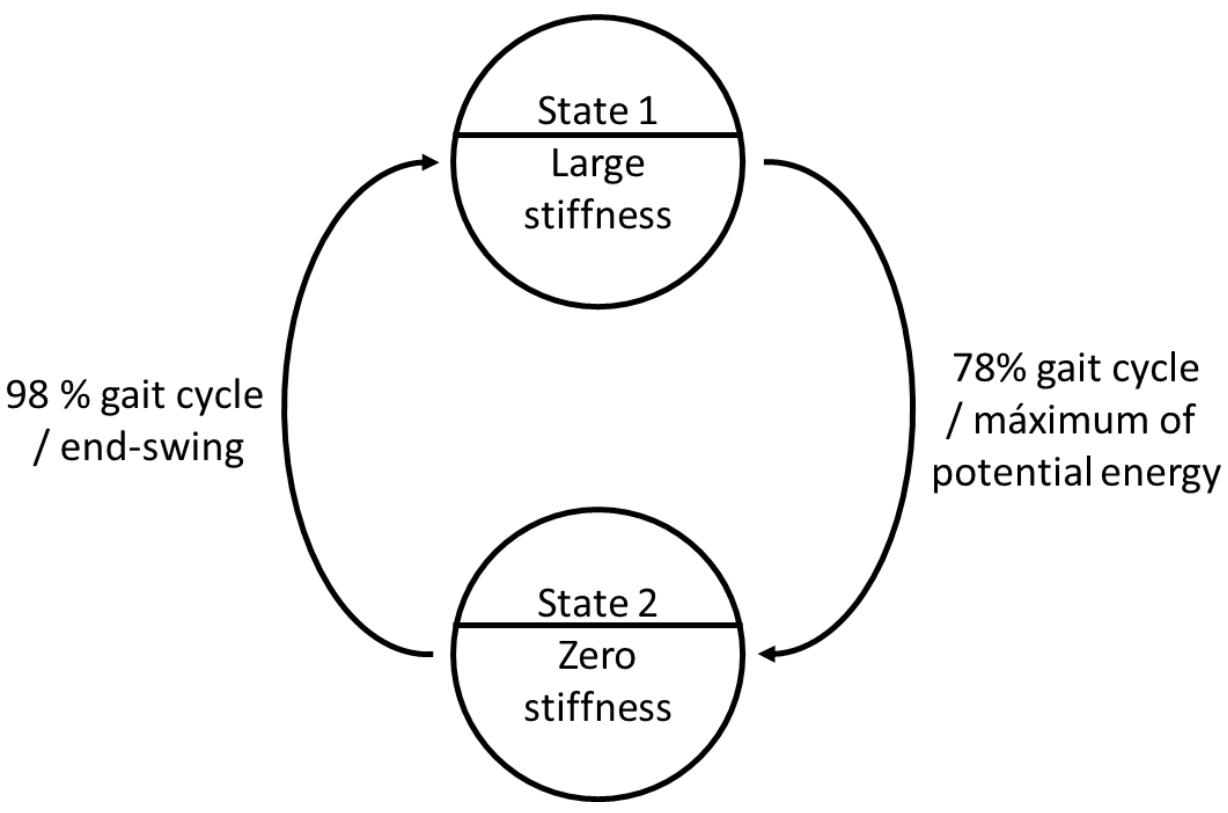

Figure 7.6: State machine controller at the hip. In each state a range of stiffness and the transition condition are shown.

Therefore, based in this analysis, the stiffness pattern implemented in the hip joint is shown in Figure 7.7

\subsubsection{Stiffness control at the knee}

From the requirements obtained from the biomechanics analysis of human gait in Chapter 2, knee stiffness variation in Section 6.2.2 and the energy efficient models proposed in Chapter 4, the following conclusions arise:

In the load response phase the energy is accumulated in the acceptance of the body weight, and this energy is released for knee extension. Therefore, in this phase the stiffness of the ARES joint is adapted both to the speed and the weight of 


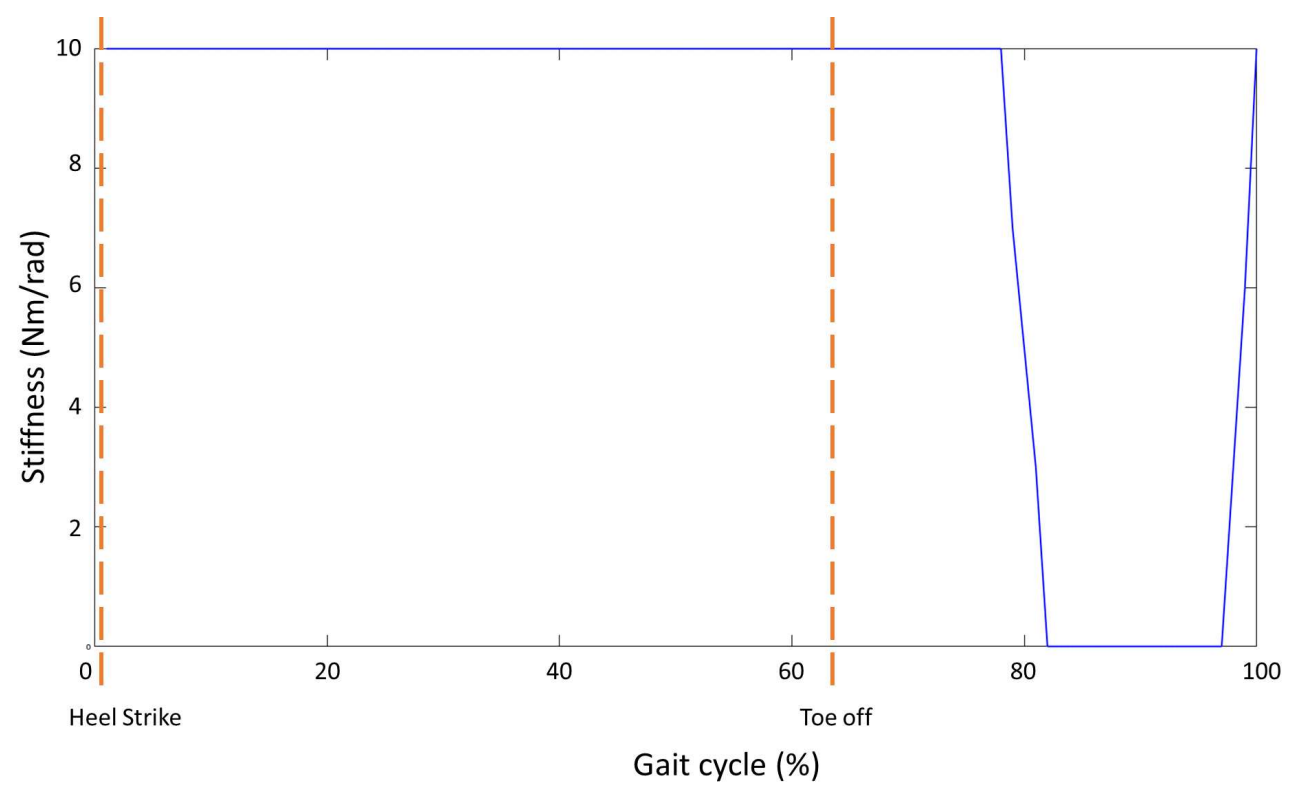

Figure 7.7: Pattern of stiffness implemented in the hip to exploit the intrinsic dynamics of the joint.

the user as explained Section 6.2.2. In the remaining of the support phase the knee required enough power to support the weight of the user.

After stance, in the initial swing the knee requires high stiffness to flex and provide ground clearance. As it is shown in Chapter 4, in the intermediate swing phase a zero stiffness is required to take advantage of the dynamics of the leg.

Again, stiffness must be increased before heelstrike, a $5 \%$ of the gait cycle, to ensure a controlled ground contact.

In order to implement the stiffness requirements in ARES the actuator in the knee, the following set of features is required, for the ARES actuator:

- Ability to achieve mechanical zero stiffness.

- Ability to store and release energy at different speeds. As discussed in Section 6.2.2 based on biomechanical study results, to accumulate energy in the springs, it is necessary to set the intrinsic stiffness of the actuator at a certain value dependent on the speed of the joint and on the weight of the user.

- Ability to generate enought required power as detailed in Section 6.1.2 
The ARES actuator meets all the required features. To examine the performance and evaluate the behavior of the actuator in each phase of the gait at the knee, the control of stiffness has been divided into subsystmes:

1. The first subsystem is based on an impedance control approach for commanding a stiffness value of $6 \mathrm{Nm} / \mathrm{deg}$ at the loading response phase without energy expenditure in motor M1.

2. The second subsystem is a state machine for the control of the ARES actuator to reduce energy consumption in the complete gait cycle.

During locomotion, a state machine switches between stiffness values for the knee joint. The state machine, (see Figure 7.8), is based on the study presented in Section 6.5[119]. Four discrete knee stiffness states are required:

State 1. After toe-off at the initial-swing phase, a large stiffness (the maximum of ARES $10 \mathrm{Nm} / \mathrm{deg}$ ) is needed to let the motor lift up the shank.

State 2. When it has reached the highest foot height during swing, zero stiffness allows exploiting the intrinsic dynamics of the limb for swinging forward and the potential energy stored when the motor changes its direction.

State 3. At the end of swing the stiffness is increased to prepare for the collision at heel strike but kept low enough to allow the actuator springs to absorb the impact energy, enabling an adequate load response to begin the stance phase. As, for the hip, the knee stiffness in this phase depends on the speed of the gait and on the weight of the user.

State 4. At the support phase the motor of the knee joint is blocked and a high stiffness is enforced to support the user weight but still providing some compliance by hte elastic components.

It is important to remark that due to the characteristics of the variable stiffness joint design, the force required to change the stiffness of the joint increases as the torque does. Therefore, to reduce the energy consumed by the motor which changes the stiffness, every modification in stiffness is performed when a reduced torque is being done. 


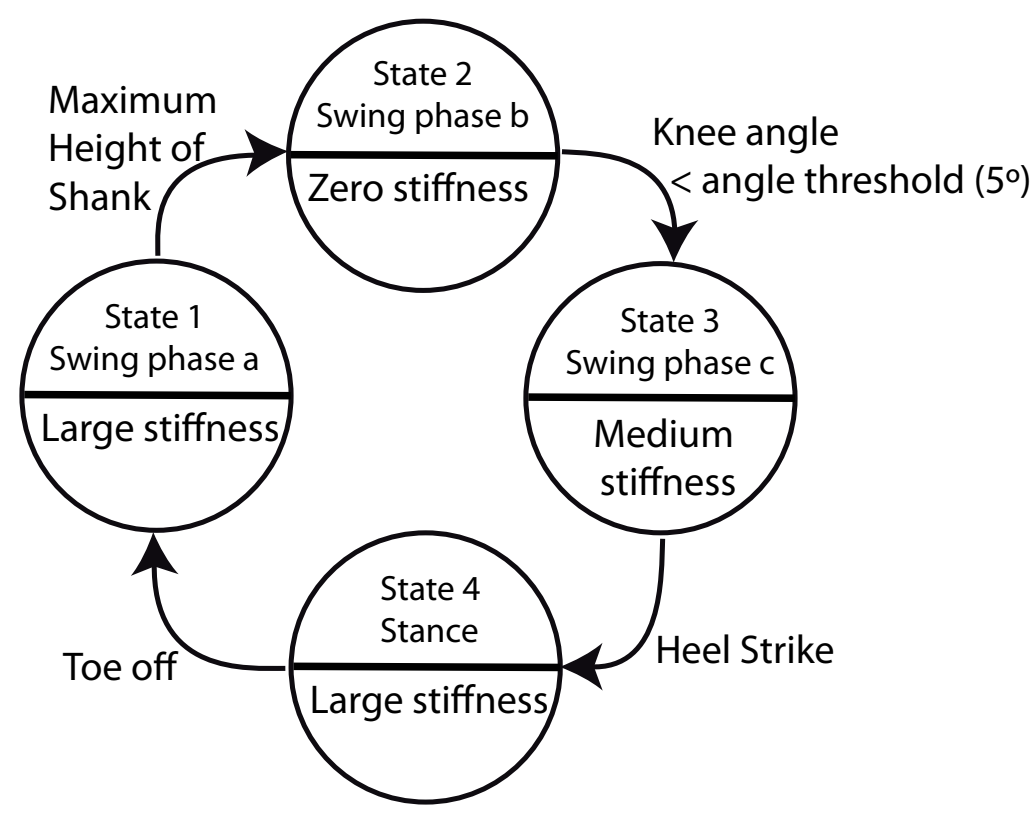

Figure 7.8: State machine controller at the knee. In each state a range of stiffness and the transition condition are shown.

Figure 7.9 represent the stiffness pattern that exploit the natural dynamics at the knee. Note how the stiffness goes to zero when the maximum in the potential energy is reached. This stiffness pattern also adapts to the motion in the loading response, because the motion of the motor $\mathrm{M} 2$ to recover the $10 \mathrm{Nm} / \mathrm{deg}$ in the initial response, produce in the joint the required motion, as it is explained in the experimental implementation in Section 7.4.4.

\subsubsection{Stiffness control at the ankle}

The control strategy implemented in the ankle is different from that implemented in the knee, similarly to human biomechanics.

In the ankle gait cycle, elastic energy is stored during the loading response and mid-stance phases and this energy is released in the toe off. Technically the complexity of this approach is that energy accumulates and is not released immediately, it is released later in the toe-off stage of the gait. During locomotion a state machine switches between stiffness value (see Figure 7.10). 


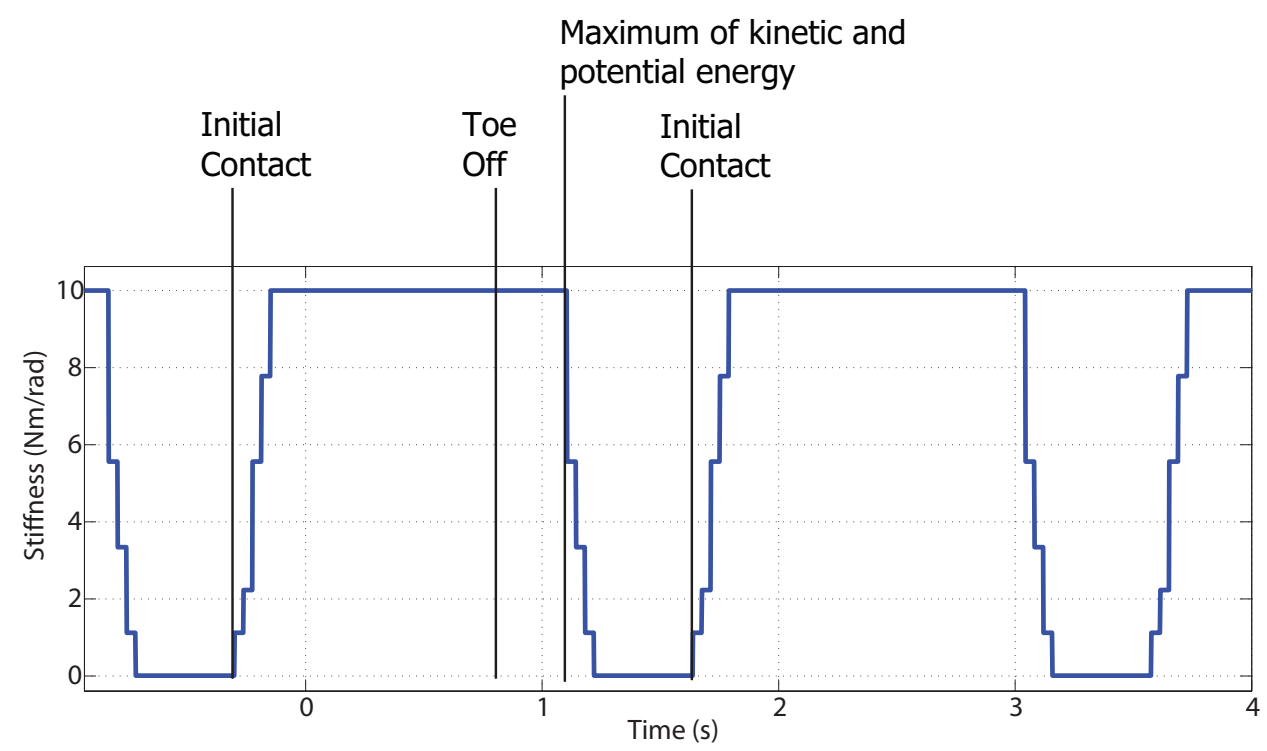

Figure 7.9: Pattern of stiffness implemented in the knee to exploit the intrinsic dynamics of the joint.

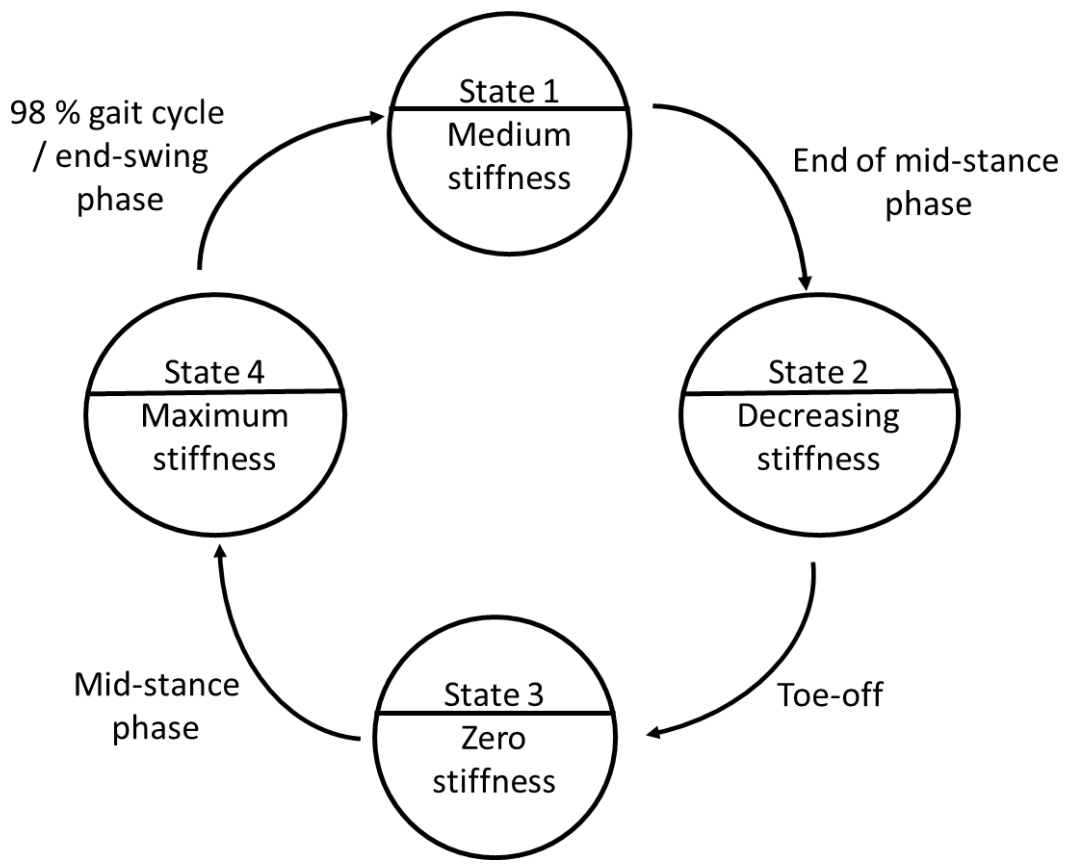

Figure 7.10: State machine controller at the ankle. In each state a range of stiffness and the transition condition are shown. 
- State 1. In the beginning of the support phase, stiffness that depends on the speed of the gait and on the weight of the user to damp the contact is selected, ( $3 \mathrm{Nm} / \mathrm{deg}$ in Figure 7.11).

- State 2. In the intermediate phase of the stance, a sufficiently low ( $3 \mathrm{Nm} / \mathrm{deg}$ ) stiffness is maintained to adapt to the terrain and accumulate energy in the springs. This low stiffness state is maintained to release the elastic energy at the appropriate moment.

- State 3. In the toe-off phase the stiffness is increased sharply, taking advantage of the energy accumulated in the springs and the movement that produces the variation of stiffness in the ankle joint.

- State 4. In the swing phase a high stiffness $(10 \mathrm{Nm} / \mathrm{deg})$ is maintained to avoid the ground until the phase prior to the contact of the foot where a low stiffness is selected to accommodate the ground impact.

Based on the analysis of the biomechanics of human gait presented in Section 6.6, the following stiffness variation strategy has been established (see Figure 7.11).

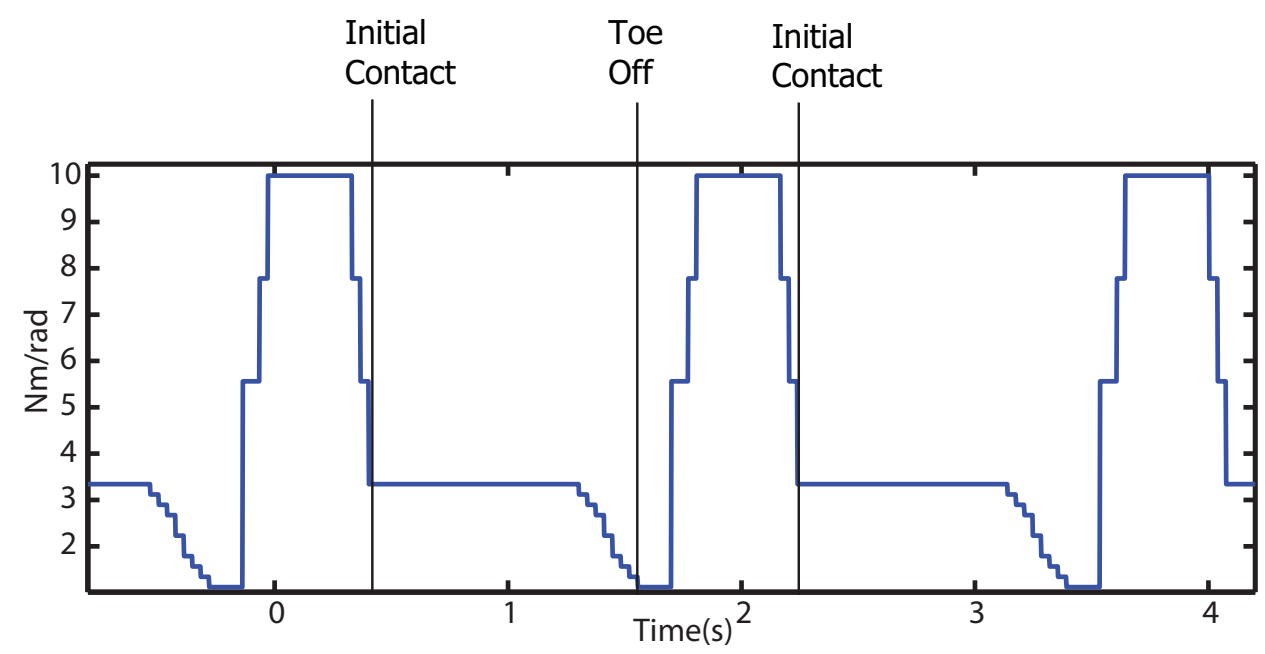

Figure 7.11: Pattern of stiffness implemented in the ankle to hold and release the energy. 


\subsection{Experimental assessment of the control approach}

\subsubsection{Experimental setup}

An experimental setup has been prepared with two aluminum bars linked to the actuator resembing an articulation of the exoskeleton. The test-bench that has been used in the evaluation of these control strategiesis shown in Figure 7.12, A load of $1.5 \mathrm{~kg}$ at the end of the actuator emulates the mass of the links.

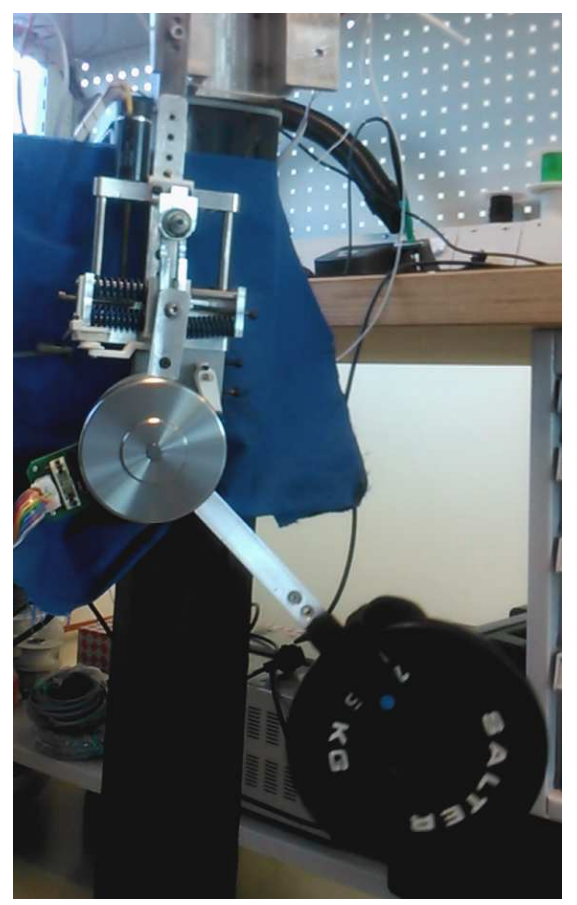

Figure 7.12: Test-bench to analise hybrid strategies.

The electronics to perform the control proposed is based on a National Instruments board controller (MyRIO) which features a real-time processor and Xilinx FPGA. Both controls of joint positionand stiffness variation is centralized in this MyRIO controller.

Figure 7.13 shows the experimental control scheme used. The main joint controller generates the required trajectories, angles and speeds for the joint and sends velocity commands to a controller via PWM to a motor, 90W DC Maxon flat of $24 \mathrm{~V}$ motor, using an ESCON 50/5 controller. The position of the joint is measured 


\section{ENERGY-EFFICIENT CONTROL OF LOCOMOTION}

with an AS5045 encoder that articulates with a precision of 12 bits. The M2 stiffness variation motor, a Maxon RE-25 AC motor of $20 \mathrm{~W}$, is controlled by an EPOS $24 / 5$ controller with closed loop based in the position of the pivot.

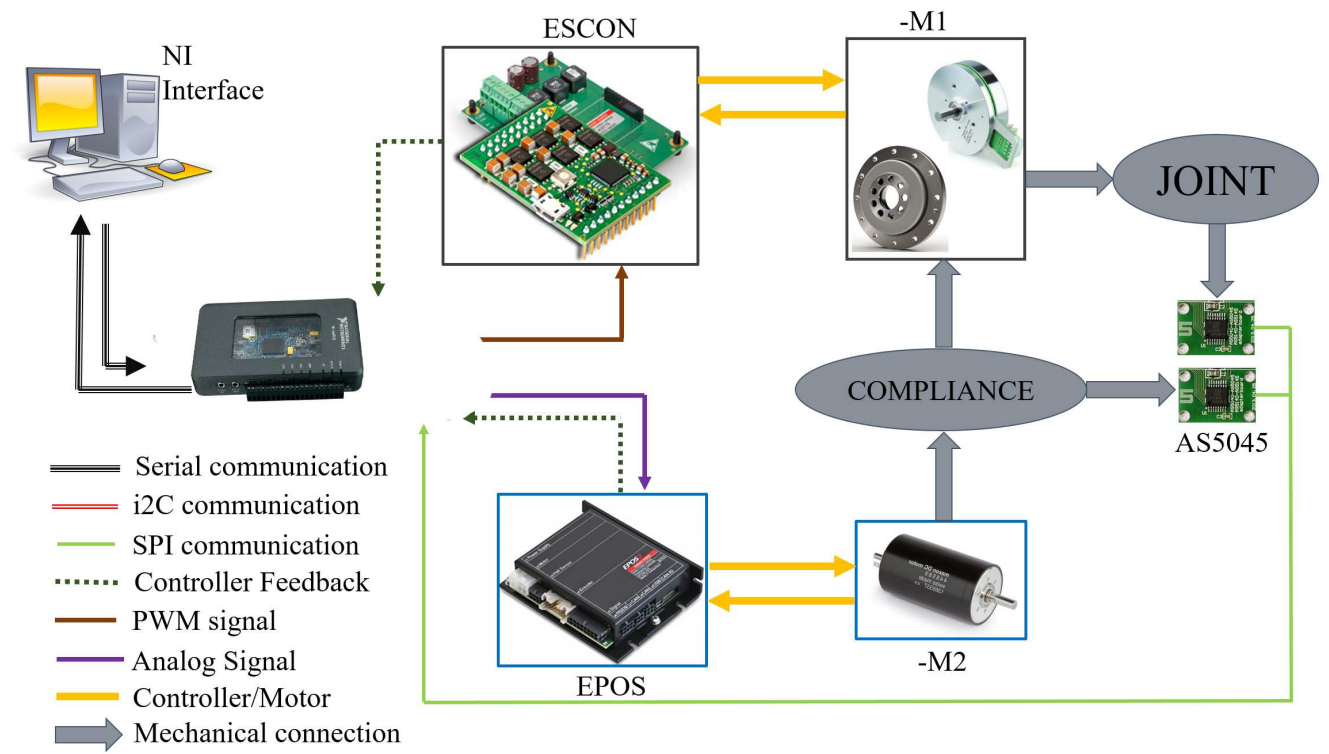

Figure 7.13: Experimental control scheme.

\subsubsection{Force measurement validation}

To evaluate the performance of the force sensor and the behavior in the force measurement system, two different loads of 1.6 to 3.6 kilograms, equivalent to the weight of the human links, were attached to the actuator and a sinusoidal trayectory was comanded to the joint. Comparing the theoretical force with the measures obtained from equation 6.13 an assessment is made.

Figures 7.14 and 7.15 show the results and a very good fit is observed in the torque measurement. Note that with low stiffness (Figures 7.14 (a) and 7.15 (a)) there is a greater delay in the measurement of the torque because a greater deflection is necessary for the measurement. With this capability of both torque measurement and stiffness variation in the following sections the strategies for the reduction in energy expenditure of both joint dynamics and actuator properties will be analyzed. 


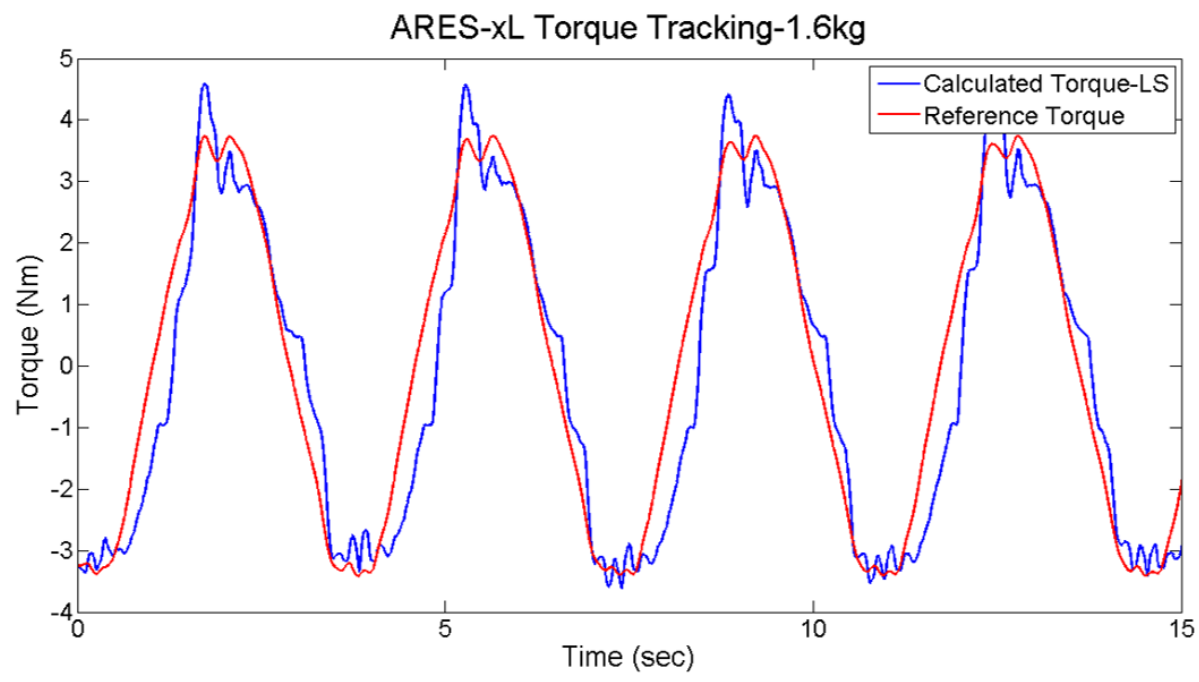

(a)

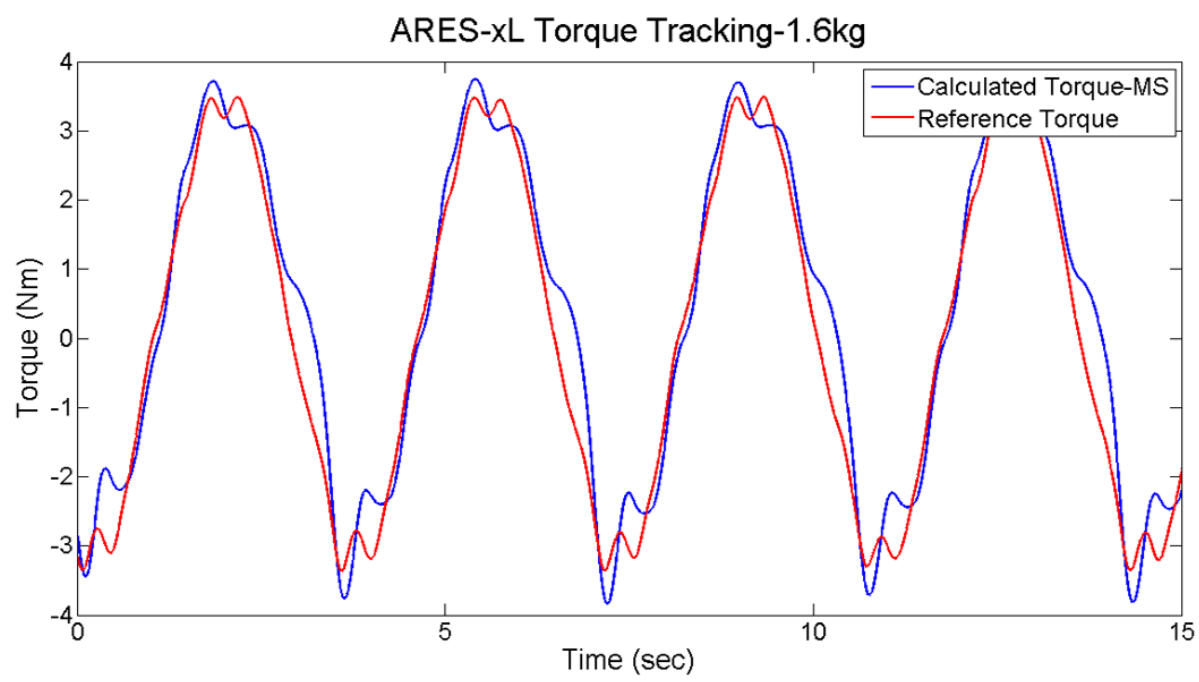

(b)

Figure 7.14: Torque Tracking in ARES at 2 Levels of Stiffness, (a) low stiffnes (2 $\mathrm{Nm} / \mathrm{deg})$ and (b) high stiffness $(10 \mathrm{Nm} / \mathrm{deg})$, with a $1.6 \mathrm{Kg}$ Load. 


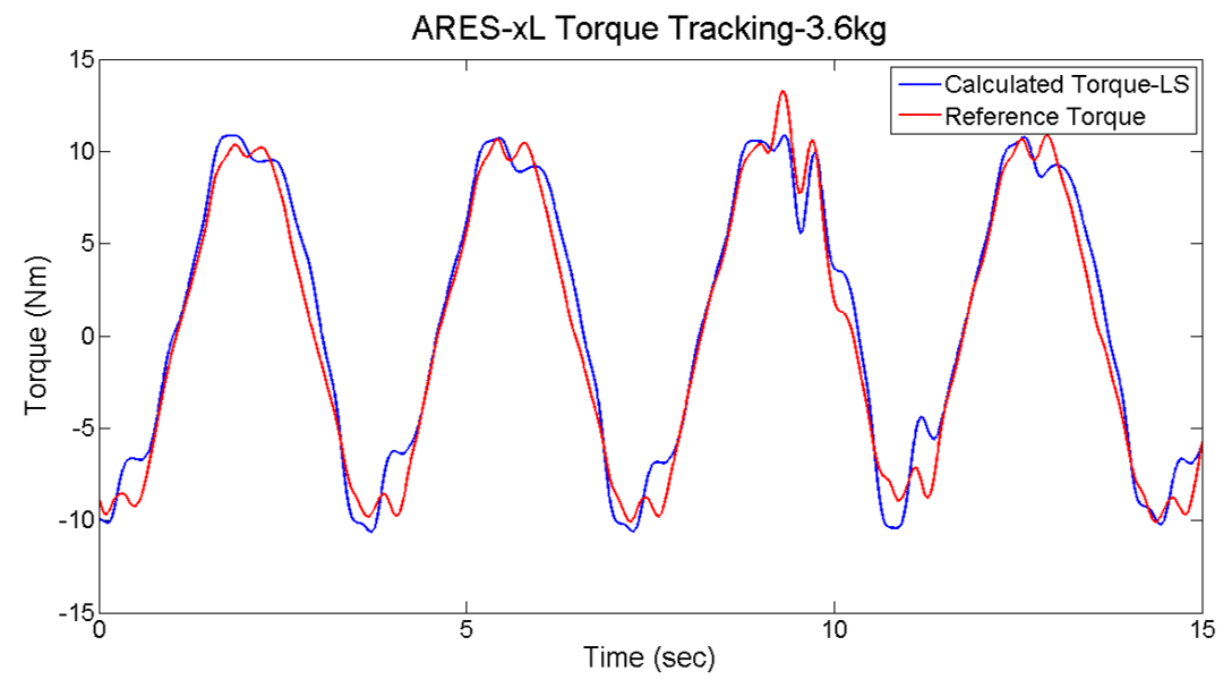

(a)

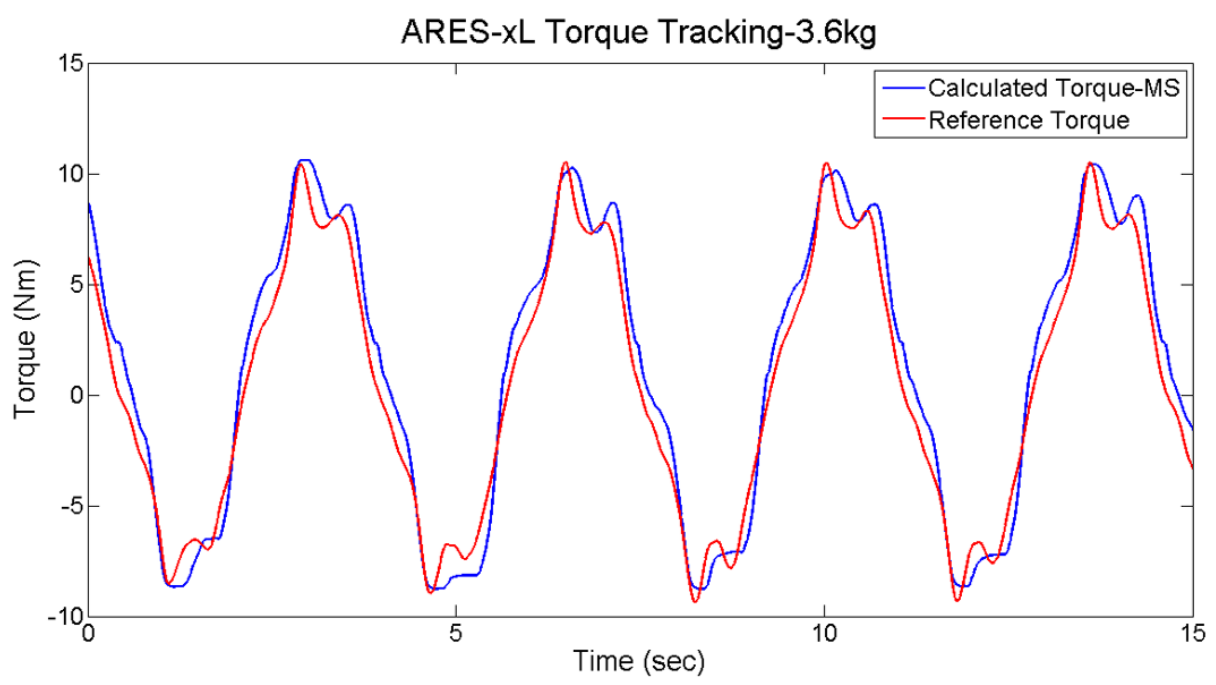

(b)

Figure 7.15: Torque Tracking in ARES at 2 Levels of Stiffness, (a) low stiffnes (2 $\mathrm{Nm} / \mathrm{deg}$ ) and (b) high stiffness (10Nm/deg), with a $3.6 \mathrm{Kg}$ Load. 


\subsubsection{Hip stiffness control}

In order to take advantage of the dynamics of the system by varying the stiffness of the ARES actuator, the motors M1 and M2 will be controlled in parallel and synchronized. M2 will control the stiffness according to a pattern established by the results of chapter 2 analysing of the human gait. While the M1 motor will follow the path required for the gait, synchronized with M2 to compensate its movements and with the rest of the joints of the active orthosis, taking advantage of their inertias and compensating their movements.

The implemented control approach reduces or cancels the current sent to the M1 motor in the phases where it is necessary to take advantage of the dynamics of the system or the energy accumulated in the springs. In these cases, the motor stays in a standby phase without consuming energy, monitoring the path followed by the subactuated joint. At the moment that this actuator returns to act, it must do it without causing sudden movements in the joint. So control of the speed of the joint is performed to maintain it continuous, that is, without jumps.

The general equation that defines the control signal (speed) sent to the motor M1 is defined in the Equation 7.1 .

The control signal (position) in the motor M2 is is defined in the Equation 7.2. This control signal is sent to the PID controller that has been implemented to follow the defined stiffness scheme. The control takes into account the variation of the angle of the joint by the motor M2 which is corrected by the motor M1.

In turn, the control signal sent to the main motor M1 follow a pattern that is null in the gait phases where it is needed to take advantage of the intrinsic dynamics of the leg or the energy accumulated in elastic elements.

\subsubsection{Experimental validation of ARES at the hip}

In this way, the stiffness pattern that has been implemented is shown in Figure 7.7. In the stance phase the highest stiffness $(10 \mathrm{Nm} / \mathrm{deg})$ is maintained while in the swing phase it is reduced to zero to take advantage of the intrinsic dynamics.

Figure 7.16 shows the joint trajectory that is followed with precision, thanks to the fact that in the control it is taken into account how the variation of stiffness also modifies the output angle. 


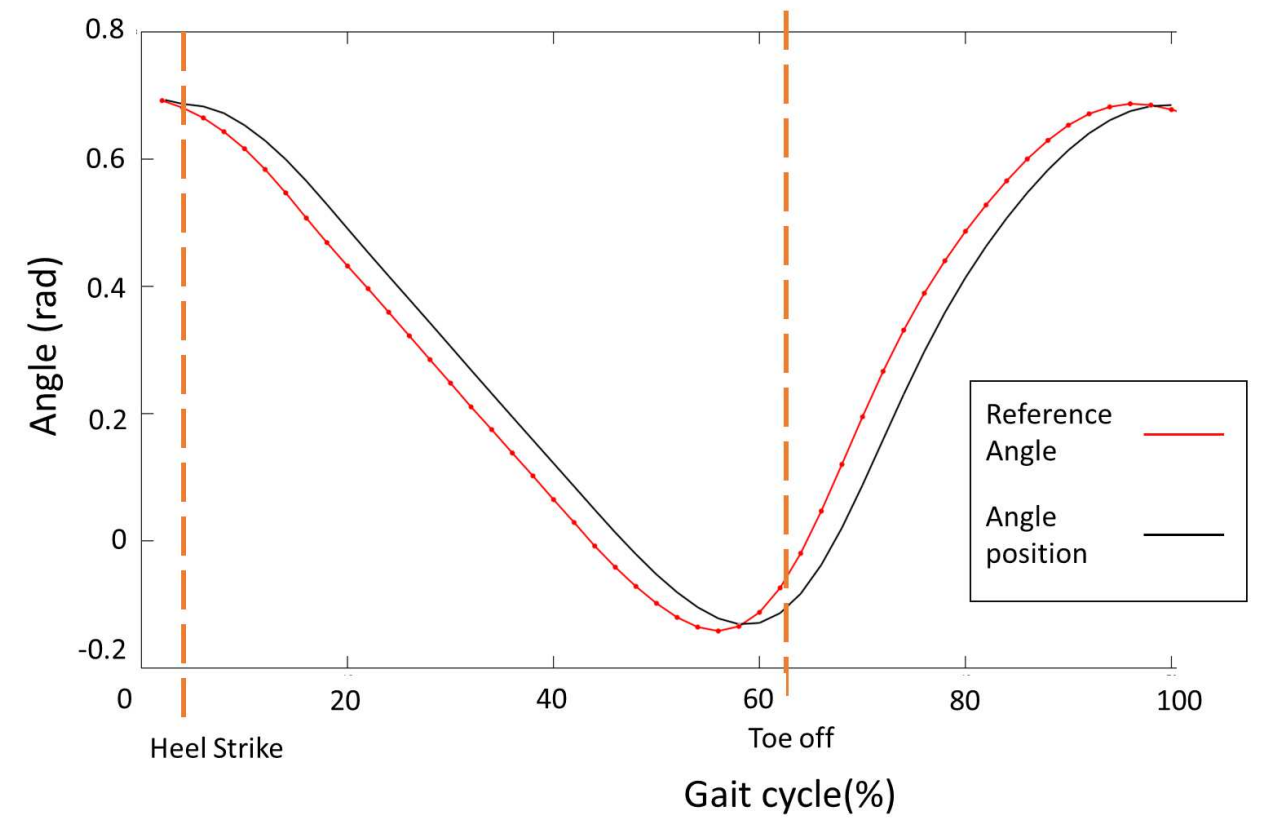

Figure 7.16: Position trajectory followed by the hip joint with proposed control varying stiffness to reduce energy consumption. 
Figure 7.17 shows the power consumption of the main actuator (M1), it is possible to observe a zero consumption during the mid-swing phase.

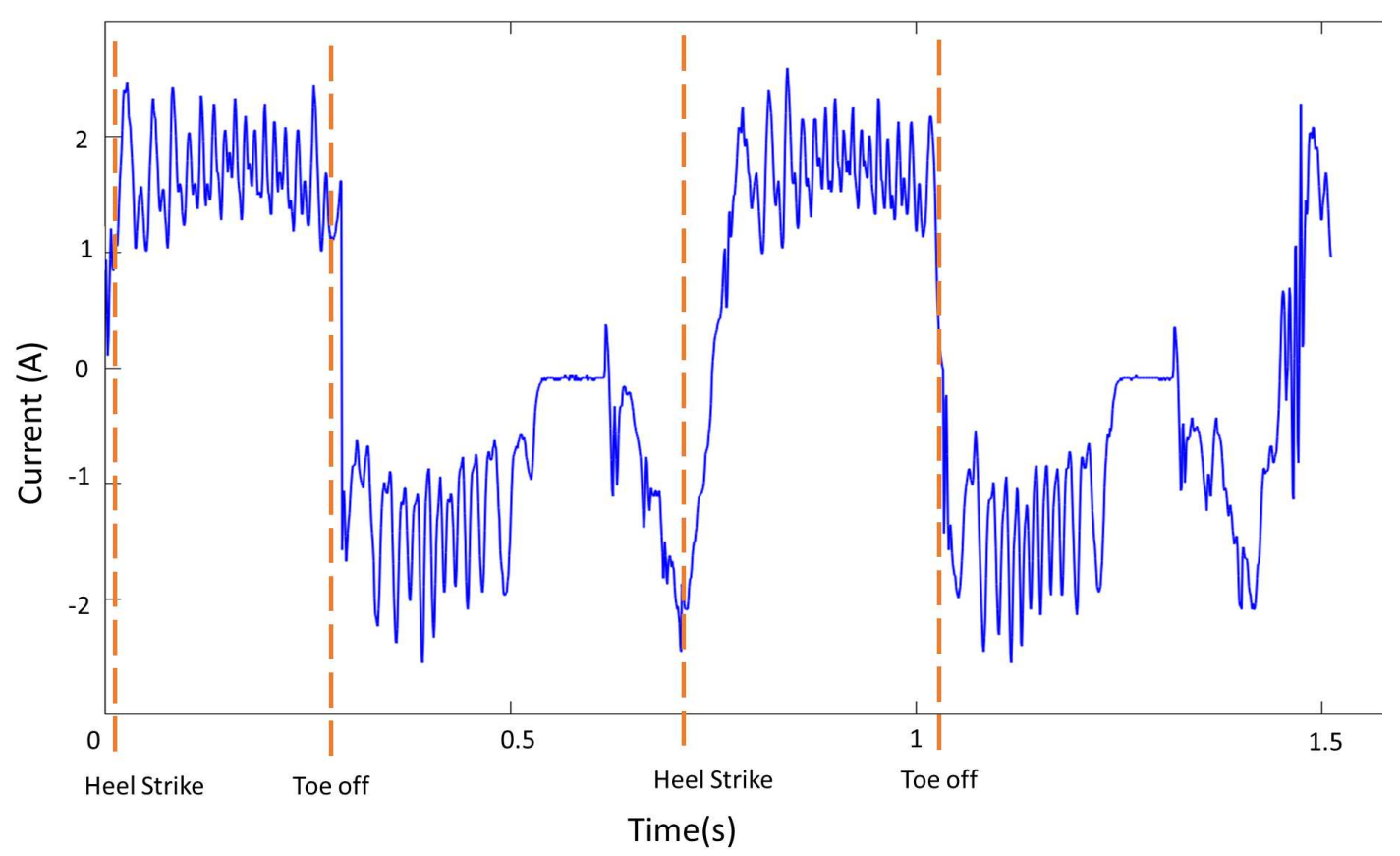

Figure 7.17: Current consumed at motor M1 varying stiffness to reduce energy following the trajectory shown in Figure 7.16 .

Applying this strategy of passive dynamics a reduction in energy consumption in the hip of $17.2 \%$ is achieved.

\subsubsection{Knee stiffness control}

In a first approximation, to evaluate the energy savings only in the load-response phase, the state 2 of swing phase of the state diagram of Figure 7.8 has not been implemented. Therefore, zero stiffness has not been established at this first analysis, and the intrinsic dynamics of the leg will not be exploited. The complete approach will be experimentaly tested in Section 7.4.4.1

Figure 7.18 shows the CGA position pattern followed by the knee and the modified pattern. 


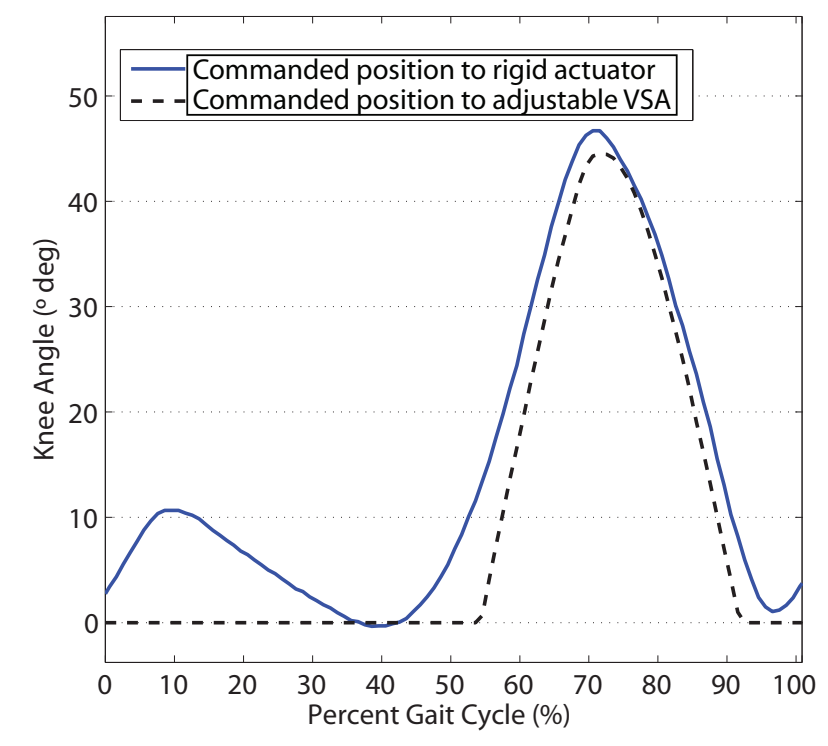

Figure 7.18: CGA pattern utilized as a position reference to the rigid actuator and the pattern commanded to the variable stiffness actuator.

The ARES prototype has been incorporated in the knee joint of the ATLAS orthosis, see Figure 7.19 and tested on a dummy. The state machine scheme proposed in Section 7.3.2 has been implemented but only has the stance phase, therefore without including State 2 (swing phase).

Figure 7.20 (a) presents the commanded position in the actuator and the real position achieved due to the compliance controlled with the state machine. In this Figure is plotted in red the original CGA only for comparison purposes. In black the trajectorie commanded to the motor is represented, while in blue is plotted the angle measured in the knee by the magnetic encoder. Notice that even without having actively commanded the hump in the heel strike joint trajectory, it is observed that load response follows that pattern due to the low impedance implemented that allows the knee to adapt to the ground during the support phase.

A comparison of the current consumption in a steady gait with a CGA pattern and rigid actuator and with the new pattern and the compliant actuator is shown in Figure 7.20 (b). Notice the power expenditure reduction in each current peak, which corresponds to changes of direction in the joint trajectory. This is because the springs accumulate and release energy at each change of direction. During the 

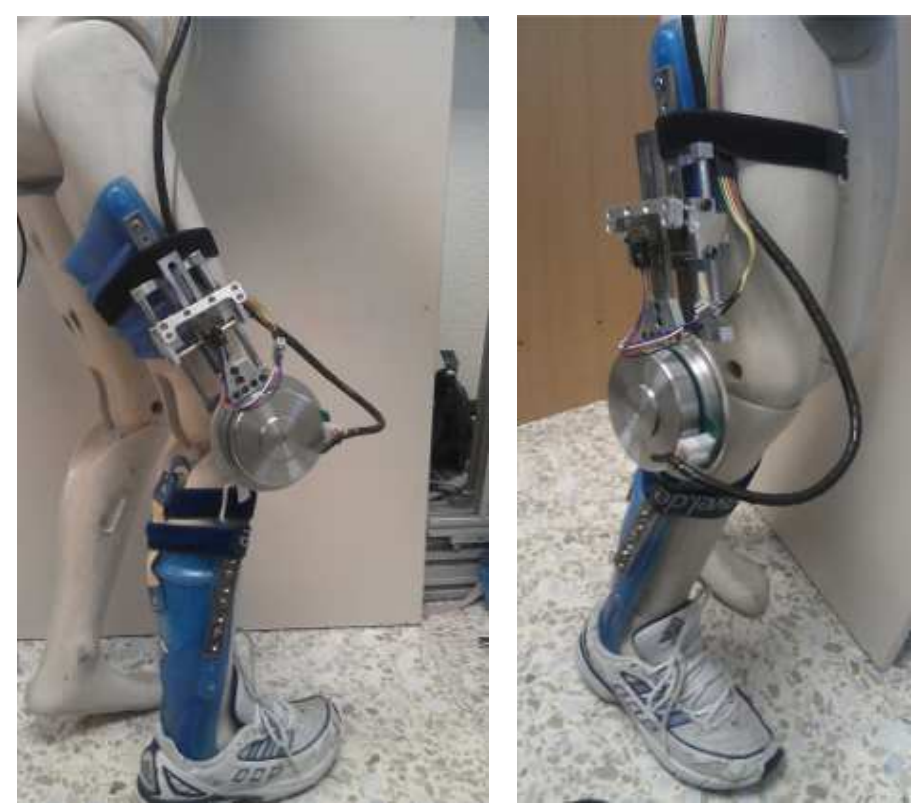

Figure 7.19: Implementation of the adjustable compliant actuator in the knee joint.

support phase, no energy is required to block the knee due to the large stiffness, the high reduction ratio in the motor-gear set and the position pattern proposed. Even so, Figure 7.20 (a) shows how the knee allows adaptation thanks to the inherent compliance of the joint.

When compared to the rigid actuator following a rigid pattern of CGA, the proposed ARES control approach achieves a reduction in energy consumption of $39 \%$. It is observed a reduction in the peak of the current when the direction of the joint changes. An important reduction is achieved keeping the knee blocked in the support phase, while providing compliance enough to accommodate the body weight.

In the next subsection, in addition to establishing adequate stiffness in the load response phase, to take advantage of the elastic behavior in the gait phase, zero stiffness is included in the mid-swing to take advantage of the potential energy and inertia of the limbs . 


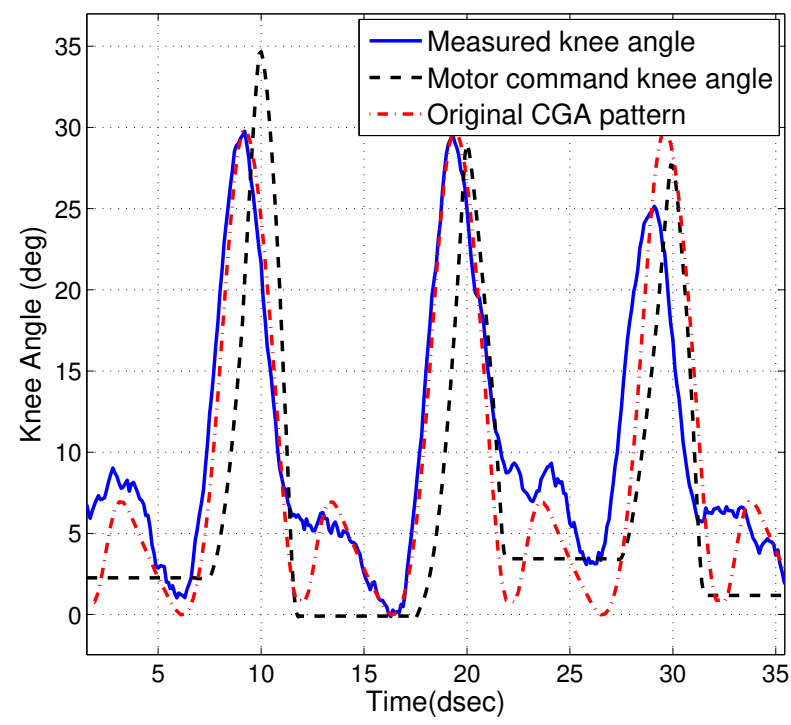

(a)

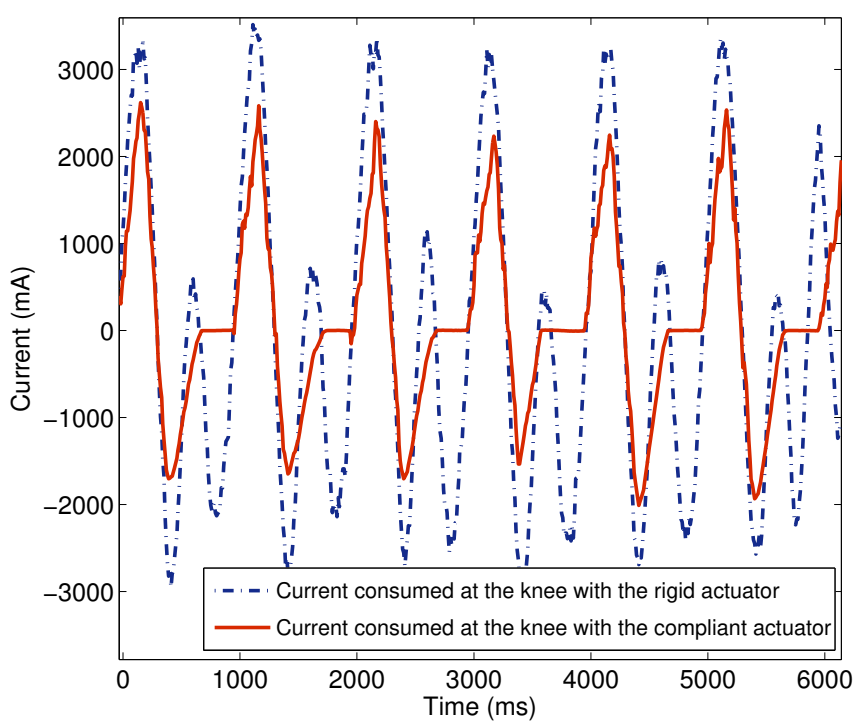

(b)

Figure 7.20: Knee stance control(a)Commanded position and the real position reached with the compliant actuator. (b) Current consumption with the rigid actuator and with the variable stiffness actuator. 


\subsection{Experimental assessment of the control approach}

\subsubsection{Complete knee gait cycle}

In order to exploit the complete strategy proposed in Section 7.3.2, the state machine of Figure 7.8 must be followed, with the hybrid control strategy presented in Section 7.2.

The stiffness variation control for the knee has been implemented in the test bench described in Section 7.4.1. In Figure 7.21 a secuence of snapshooots of the experiment is shown. The knee is commanded to move in a complete gait cycle. 


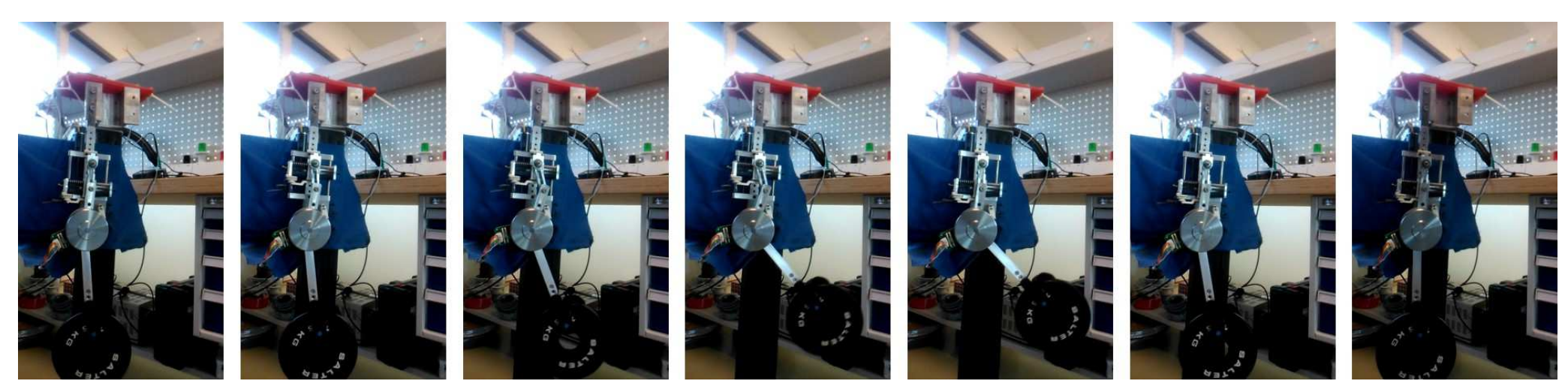

Figure 7.21: Sequence of the snapshoots with the impemented strategies varying stiffness. 
The energy consumption with the control strategies proposed in ARES is compared to the rigid approach described in Appendix A.

Thus, throughout the gait cycle, a stiffness variation scheme is performed as proposed, in Section 7.3.2 (see Figure 7.9.)

In the initial contact at the heel strike phase the stiffness is reduced abruptly to zero both to take advantage of the stiffness obtained in the load response and to take advantage of the movement of the angular position of the output of the joint with the motion of the actuator M2 defined in Equation 6.8. During the remaining of the cycle up to mid-swing, a high stiffness is maintained to perform a good trajectory tracking. In the mid-swing, which corresponds in the knee to the phase of maximum potential energy, stiffness is reduced to zero to exploit the intrinsic dynamics of the leg. Both in this phase and in the loading response phase, the main actuator M1 does not actuate, obtaining the desired angular movement from the stiffness variation.

Let remark that it has been taken into account, as stated in Equation 6.8, that the variation and adaptation of stiffness produces a variation in the angle of the joint itself that not only must be taken into account to be compensated, but also can be used for the movement of the main actuator of the joint not requiring to consume energy. This is very noticeable after the transition from the lower stiffness phase to the high stiffness phase prior to leg support. In this moment maximum deflection with null stiffness occurs, as shown in Figure 7.21. When the actuator M2 increases the stiffness, increases the angle of the joint significantly, just as the joint moves in the load response phase. Therefore, in this phase the motor M1 will consume little power (Figure 7.23) because it takes advantage of the movement produced when the stiffness is modified.

As discussed in Section 7.3.2, all these strategies should be performed by maintaining the joint speed as a continuous function.

Figure 7.22 shows the position tracking of the joint while following the control of stiffness variation during three gait cycles. It is observed that even in the mid-swing phase where the stiffness varies from zero to $10 \mathrm{Nm} / \mathrm{deg}$ to prepare the support phase, the position does not suffer sudden jumps.

Figure 7.23 shows the current consumption of the main motor of the joint, actuator M1, maintaining the constant stiffness (in blue) and implementing the strategy 


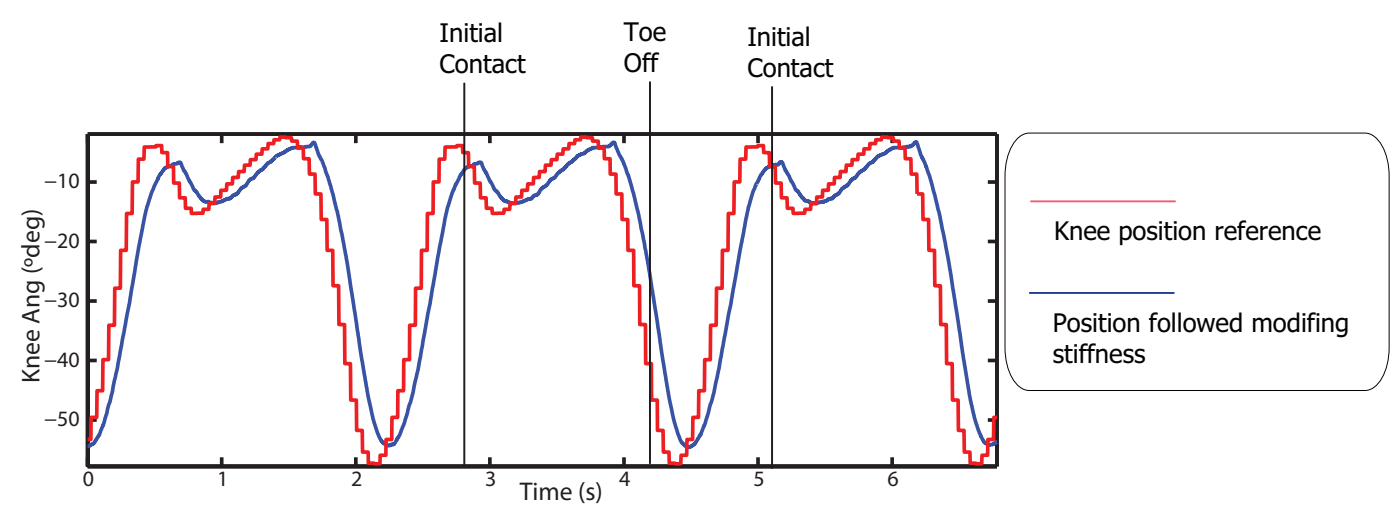

Figure 7.22: Position followed with proposed control varying stiffness to reduce energy consumption during three gait cycles.

of variation of stiffness to exploit the dynamics of the ARES (in red) during three gait cycles.

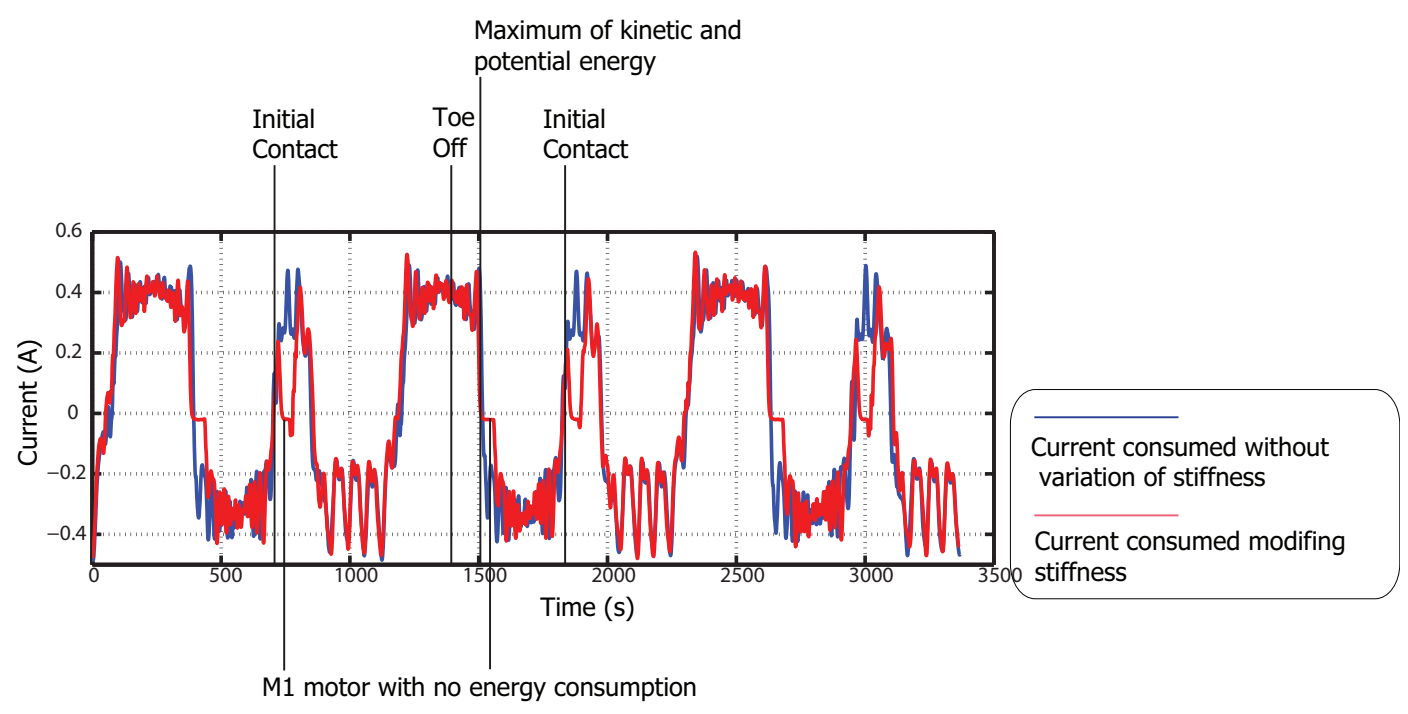

Figure 7.23: Current consumed at motor M1 without variation of stiffness (blue) and varying stiffness to reduce energy consumption (red).

It is observed from the figure that the power consumption in the intermediate phase of the swing as well as in the stance the current consumption is neglected by the control strategy of variation of stiffness.

In order to compute the total current consumption, in addition to the main motor M1 consumption, the consumption of the stiffness variation motor M2 must be 
taken into account. Figure 7.24 shows the current consumption of this actuator along a gait cycle.

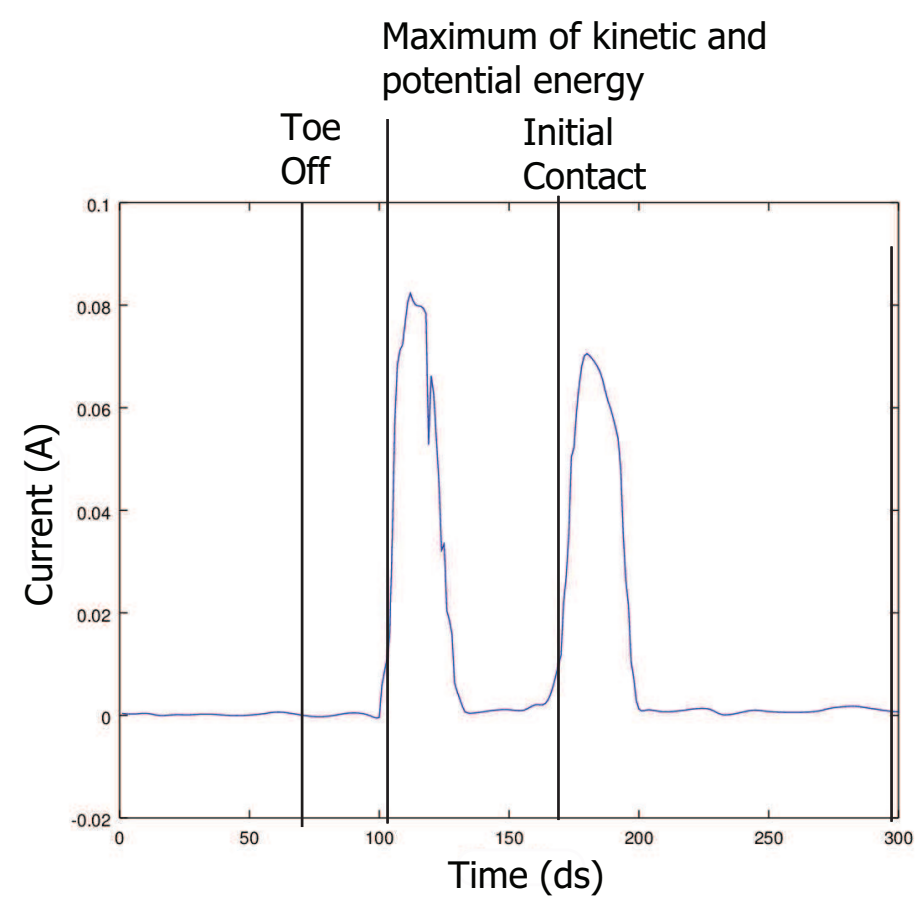

Figure 7.24: Current consumed in the motor M2 that change stiffness at the knee joint

It is observed that the order of magnitude of the current consumed by the motor of variation of stiffness (M2) is about a hundred times smaller than that of the main motor of the joint (M1). Therefore, the proposed strategies of variation of stiffness for the reduction of energetic consumption are validated.

Summing up all the terms of consumption of both engines with the strategy of control of variation of stiffness and comparing with the consumption in case of constant stiffness $(10 \mathrm{Nm} / \mathrm{deg})$, a reduction in the energy consumption of $10 \%$ is achieved.

\subsubsection{Ankle stiffness control}

The stiffness variation control for the ankle has been implemented in the test bench of Figure 7.25. The proposed approach is validated by comparing the behavior and the energy consumption of a constant stiffness $(10 \mathrm{Nm} / \mathrm{deg})$ and a variable stiffness 


\section{ENERGY-EFFICIENT CONTROL OF LOCOMOTION}

approach, following a joint path of CGA in the ankle. Both the joint position and the current consumption of each motor are compared.

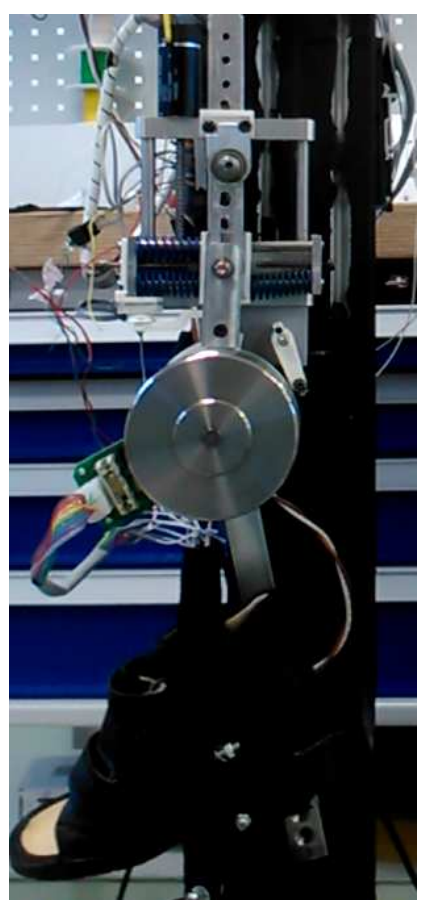

Figure 7.25: Test bench for the impemented strategies varying stiffness in the ankle.

Figure 7.26 shows (in blue) the position tracking in the case of variable stiffness, while the pattern of variation of ridigity proposed (Figure 7.11) is followed. The Figure shows (in green) the position tracked with constant stiffness $(10 \mathrm{Nm} / \mathrm{deg})$ and the reference position (in red).

It is observed that, with the proposed control strategy, a good position tracked is achieved without jumps in the derivative. The main motor of the joint adapts to changes in stiffness and impacts with the ground.

In Figure 7.27, the current consumption of the main motor M1 with variable stiffness (in blue) and with constant stiffness (in green), in a cycle of the gait, is shown. In the graph obtained for the variable stiffness approach, it is observed that during the whole phase of push-off current consumption is zero. The energy accumulated in the springs together with the inertia introduced by the variation of stiffness provide the needed movement. Since it is during that push-off phase when more power is consumed sSee Chapter 2) this strategy provides very good results. 


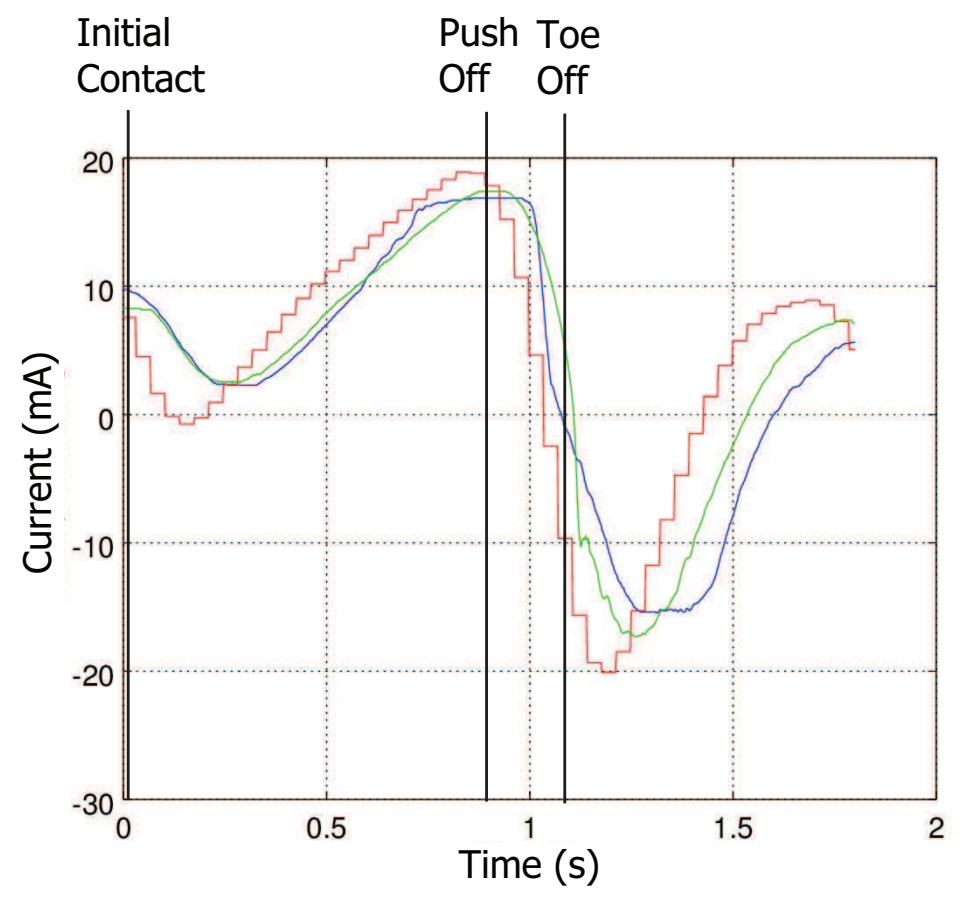

Ankle Position reference

Position followed modifing stiffness

Position followed without variation of stiffness

Figure 7.26: Position followed with a control varying stiffness strategy to reduce energy consumption in blue, with fix stiffness $10 \mathrm{Nm} / \mathrm{deg}$ in green and position reference in red. 


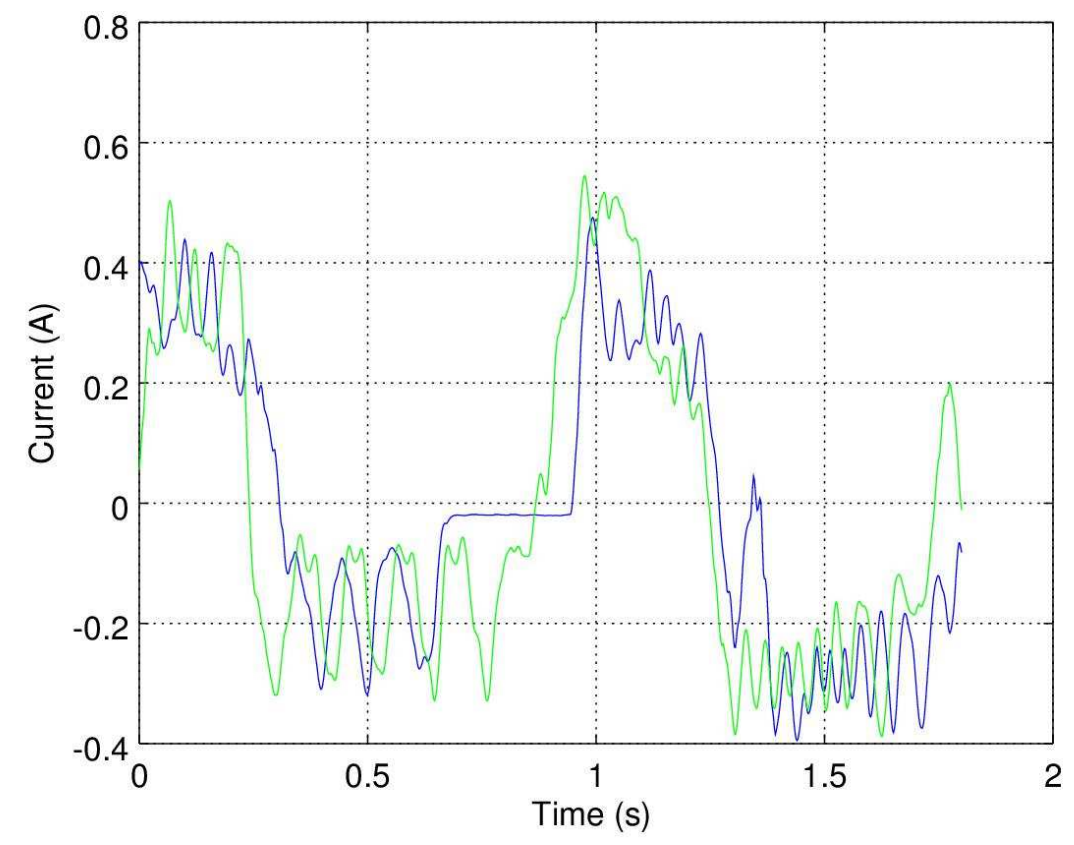

Figure 7.27: Current consumed without variation of stiffness (green) and varying stiffness to reduce energy consumption (blue). 
The power consumption of the stiffness variation motor M2, shown in Figure 7.28 , is taken into account in the total amount of energy consumed.

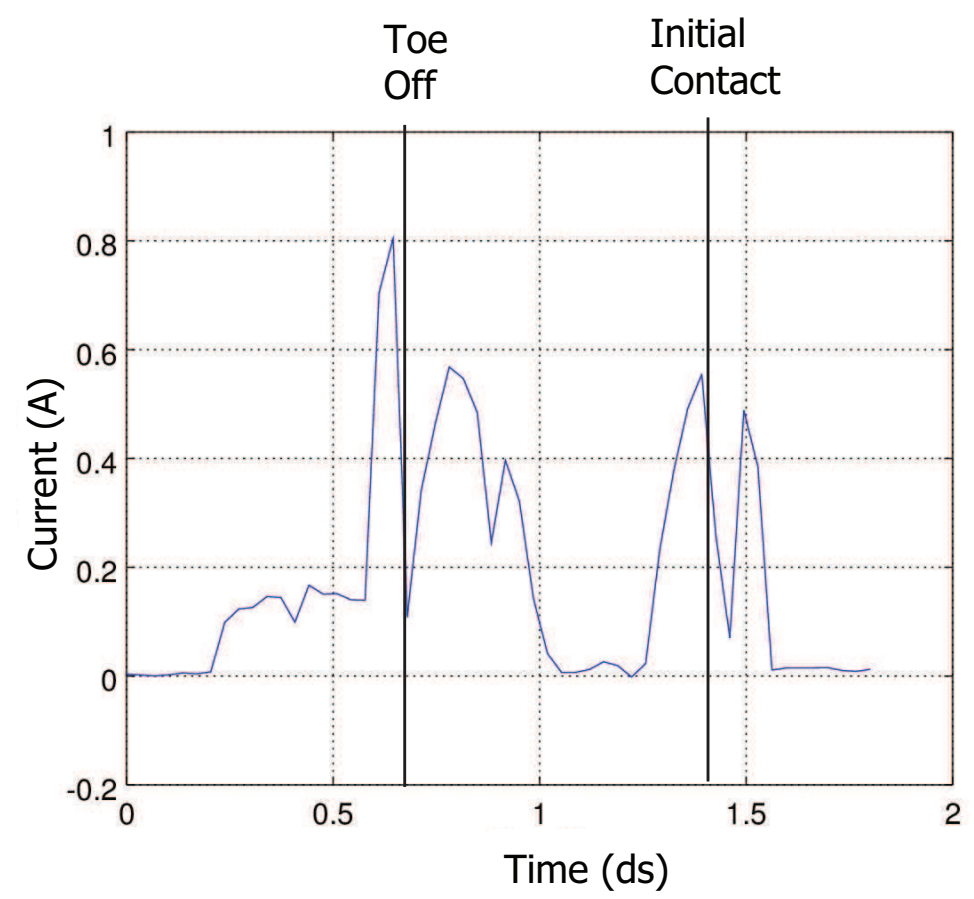

Figure 7.28: Current consumed in the motor M2 that changes stiffness at the ankle joint

The power consumption in this motor is practically negligible compared to the main motor M1.

Taking into account the consumption of both motors with the strategy of variation of stiffness and comparing the consumption with that of the strategy of fixed stiffness, a significant saving of the energy consumption of $22.6 \%$ is obtained.

\subsubsection{Variable stiffness control in the ATLAS active orthosis}

In this chapter, a variable impedance actuator ARES with high variability of stiffness and a hybrid strategy to control it at the hip, the knee and the ankle joint of an active orthosis to reduce the total energy consumption has been presented.

The application of the variable stiffness control approach at the hip takes advantage of the free motion in the direct pendulum of the leg. 


\section{ENERGY-EFFICIENT CONTROL OF LOCOMOTION}

With variable stiffness control, the knee takes advantage of the inertia and potential energy in the intermediate phase of the swing while establishing the appropriate stiffness according to the speed and weight of the user to harvest energy in the phase of load response.

In the ankle the VSA is used to accumulate energy in the initial phases of the support phase and to release it in the final phase of support, the toe-off.

In all separate cases, there is a reduction of energy consumption when the joint changes direction, thanks to the contribution of the elastic elements.

These characteristics and the proposed control strategies have been used in the ATLAS complete exoskeleton to validate the combined contribution of all the joint when controlled sincronizedly in a gait phase. The ATLAS prototype is shown in Figure 7.29. Combining the consumption of the joints the overall consumption of the complete exoskeleton is reduced by more than $45 \%$.
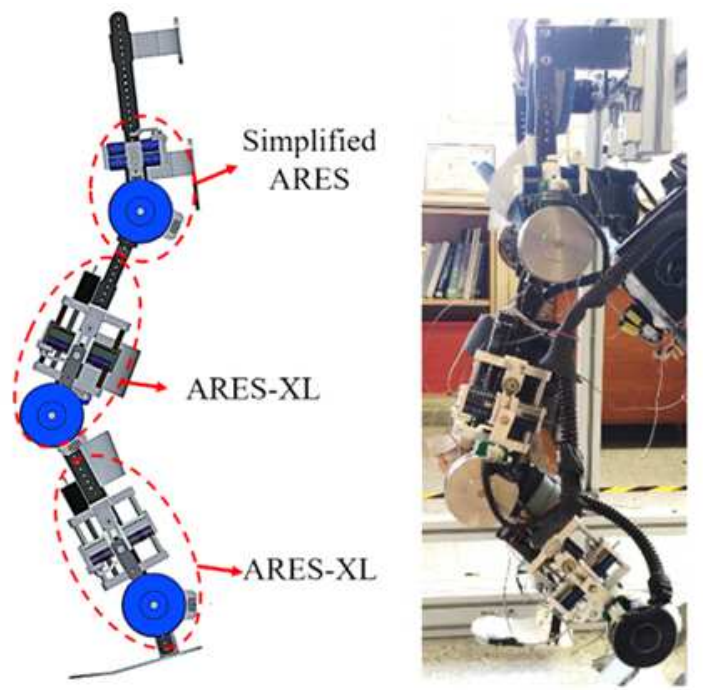

Figure 7.29: Compliant Actuation in ATLAS Exoskeletons

Recalculating the cost of transport (COT) of ATLAS exoskeleton from the equation 7.3 , since the energy consumption has been reduced by $45 \%$, the weight has increased 145 grams per actuator, the COT is at 0.52 . As Table 7.1 shows, this value is considerably lower than the rest of the exoskeletons on the market.

$$
\varepsilon=\frac{E}{W L}=\frac{P}{W v}
$$


Table 7.1: Cost of transport of several active orthoses

\begin{tabular}{|l|l|l|l|l|l|}
\hline$\varepsilon_{\text {Rewalk }}$ & $\varepsilon_{\text {REX }}$ & $\varepsilon_{\text {Ekso }}$ & $\varepsilon_{\text {Indego }}$ & $\varepsilon_{\text {Mina }}$ & $\varepsilon_{\text {ATLAS }}$ \\
\hline 1.19 & 19.8 & 0.93 & 2.76 & 6.21 & 0.52 \\
\hline
\end{tabular}

\subsection{Conclusions}

In this chapter a prototype of variable stiffness actuator has been developed: ARES (Adjustable Rigidity with Embedded Sensor) actuator is descreibed. Also an hybrid control strategy that allows the features of these actuators to be exploited. Therefore, with this actuator a strategy has been implemented for the motion control of hip, knee and ankle joints. This strategy of variable stiffness control approach have been implemented in the hip in which the natural dynamics is exploit when the maximum in the potential energy is reached, achieving a reducion in power consumption of $17 \%$.

In the knee joint, it is exploited the accumulation of energy in elastic elements in the load response phase. Appliyng this strategy achieved a consumption reduction of around $39 \%$. In the second experiment with the ARES acting on the knee, zero stiffness can be maintained, over a wide angle range. A hybrid control strategy in the knee that exploits the intrinsic dynamics of the leg has been presented. This approach increased the reduction in energy consumption by $10 \%$ in the knee joint.

The proposed hybrid control of ARES has been implemented in the ankle joint, storing energy in the support phase of the gait cycle, and maintaing it and releasing it in the push-off phase. This strategy achieves a reduction in energy consumption of $22 \%$ in the ankle joint.

By combining all these strategies in the ATLAS active orthosis, a reduction in energy consumption of $45.5 \%$ is reached compared to the conventional stiff approach. 

Now this is not the end. It is not even the beginning of the end. But it is, perhaps, the end of the beginning.

Winston Churchill

\section{Conclusions}

Human bipedal walking has evolved over millions of years to become extremely efficient. On the other hand, bipedal robots do not achieve as much efficiency unless they are passive at a cost of versatility. Thus, in bipedal robotics, a paradigm of versatility and efficiency is reached. The most versatile bipedal robots consume a lot of energy while those of passive dynamics are few versatile. The double requirement of energy efficiency and versatility is found in some robotics applications, such as exoskeletons and active orthosis for human gait assistence.

With this background, in this $\mathrm{PhD}$ work a hybrid strategy to reduce energy consumption in active orthosis has been developed and implemented. Taking the biomechanics of human gait as a reference model and taking into account the state of the art in robotics, a series of strategies have been developed that allow a significant reduction in the energy consumption of these exoskeletons.

Exoskeletons currently, require a lot of power in their joints, to move and support the weight of a human. But their actuators must be light and not much bulky, since the exoskeleton is required to be portable. Therefore, the designs that exist are based on electric motors composed of a motor that is capable of providing the requiered speed and a gearbox of much reduction ratio that converts said speed into torque. This large reduction ratio makes the joints very stiff. In turn, using this gearing cancels out the intrinsic dynamics of the exoskeleton-human limbs. 


\section{CONCLUSIONS}

Both humans and the most efficient bipedal robots take advantage of such inherent dynamics to achieve an energy efficient gait.

In the human limbs, synergy is used in the movement of one joint relative to another, with biarticular muscle-tendon systems. In this thesis, emulating biomechanics, a mode of passive actuation by exotendons to the ankle has been implemented, where in a synergic way this joint is actuated by the combinend movement of hip and knee. This allows an underactuated system, which significantly reduces not only the energy consumption but also the weight of the device. For the implementation of this concept in an active orthosis, a mechanism for coupling and decoupling the exoskeleton would be necessary since the underactuated system only performs correctly for the periodic gait and not for other tasks like getting up and sitting down.

Similarly, both humans / mammals and more efficient robots use elastic mechanisms to accumulate energy in phases of walking in favor of gravity and release it in unfavorable phases as an energy saving method. Based on simulation, it has been observed that, in order to reduce energy consumption, the stiffness of the exoskeleton joints must be varied.

For this purpose, a prototype of variable stiffness actuator has been developed: ARES (Adjustable Rigidity with Embedded Sensor) actuator, as well a hybrid control strategy that allows the characteristics of these actuators to be exploited. ARES achieves a range of stiffness close to that of the human muscle. Therefore, with this actuator a strategy has been implemented for the motion control of hip, knee and ankle joints.

This strategy of variable stiffness control approach have been implemented in the hip in which the natural dynamics is exploit when the maximum in the potential energy is reached. With this strategy a reducion in power expenditure of $17 \%$.

In the knee joint, it is exploited the accumulation of energy in elastic elements in the load response phase. Depending on the velocity and mass of the user of the exoskeleton the stiffness needed to store and release the energy in a suitable way varies, therefore, taking advantage of the stiffness variation characteristics of the actuator. This strategy achieved a consumption reduction of around $39 \%$. In the second experiment with the ARES acting on the knee, zero stiffness can be maintained, over a wide angle range. A hybrid control strategy in the knee that 
exploits the intrinsic dynamics of the leg has been presented. Both joint position and stiffness have beed commanded in the stance phase, while in the swing phase, it allowed taking advantage of the potential gravitational energy and the inertia of leg, establishing zero stiffness. This approach increased the reduction in energy consumption by $10 \%$ in the knee joint.

The proposed hybrid control of ARES has been implemented in the ankle joint, storing energy in the support phase of the gait cycle, and maintaing it while varying the stiffness and releasing it in the push-off phase. This strategy achieves a reduction in energy consumption of $22 \%$ in the ankle joint.

By combining all these strategies in the ATLAS active orthosis, a reduction in energy consumption of $45.5 \%$ is reached compared to the conventional stiff approach. This result substantially improves the autonomy of the active orthoses. If in ATLAS active orthosis the duration of a battery pack is 2 hours, the application of these rigidity variation strategies would increase its duration to almost 3 hours.

\subsection{Main contributions}

This thesis defines a framework for strategies to reduce the energy consumption in the motoin control of exoskeletons and active orthoses. As it has been seen in the development of the thesis, in order to be able to approach these strategies it was necessary to modify the philosophy of the actuators of the exoskeleton. A variable stiffness actuator has been developed for this purpose in the frame of the ATLAS project, which has been patented. The algorithms developed in this thesis allow exploiting potential energy accumulated both gravitational and accumulated in the springs for different speeds of the gait as different weights of the user, taking advantage of the variables stiffness actuation. The main scientific contributions of this thesis are:

1. A model of energy efficiency locomotion.

2. A proposal for synergic actuator with exotendons.

3. An hybrid control approach for variable stiffness actuator reducing energy consumption. 


\subsubsection{Publications}

The contributions of this thesis have been published in scientific journals of high impact (JCR).

\subsubsection{Journal publications}

1. Daniel Sanz-Merodio, Manuel Cestari, Juan Carlos Arevalo and Elena Garcia. Generation and control of adaptive gaits in lower-limb exoskeletons for motion assistance Advanced Robotics. Volume 28, Issue 5, 2014

2. Daniel Sanz-Merodio, Manuel Cestari, Juan Carlos Arevalo and Elena Garcia. Exploiting joint synergy for actuation in a lower-limb active orthosis, Industrial Robot, An international Journal. Volume: 40, Issue: 3, 2013.

3. Daniel Sanz-Merodio, M. Cestari, J.C. Arevalo and E. Garcia. Control Motion Approach of a Lower Limb Orthosis to Reduce Energy Consumption International Journal of Advanced Robotic Systems. (In press).

4. Daniel Sanz-Merodio, Elena Garcia, Pablo González de Santos. Analyzing energy-efficient configurations in hexapod robots for demining applications Industrial Robot 39(4): 357-364, 2012

5. Manuel Cestari Soto, Daniel Sanz Merodio, Juan Carlos, Arevalo and Elena Garcia. An Adjustable Compliant Joint for Lower-Limb Exoskeletons. IEEE/ASME Transactions on Mechatronics 2015

6. Manuel Cestari, Daniel Sanz-Merodio and Elena Garcia. Preliminary Assessment of a Compliant Gait Exoskeleton Soft Robotics. Vol. 4, No. 2, pp. 135-146, 2017

7. Manuel Cestari, Daniel Sanz-Merodio, and Elena Garcia. A New and Versatile Adjustable Rigidity Actuator with Add-on Locking Mechanism (ARESXL). Actuators, Vol. 7, No. 1, 2018 


\subsubsection{International Conference publications}

These contributions have been also presented in international conferences, having obtained 8 awards and recognitions for their quality and scientific and technical excellence.

- Daniel Sanz-Merodio, Manuel Perez, Manuel Prieto, Juan Sancho and Elena Garcia. Result of clinical trials with children with spinal muscular atrophy using the atlas 2020 lower-limb active orthosis Proceedings of the 20th International Conference on CLAWAR 2017

- Daniel Sanz-Merodio, Manuel Perez, Juan Sancho and Elena Garcia. Control architecture of the atlas 2020 lower-limb active orthosis 19th International Conference on Climbing and Walking Robots.Sidney, 2016. (Winner of conference best paper)

- Daniel Sanz-Merodio, Manuel Perez, Elena Garcia and Manuel Cestari. Mechanical description of atlas 2020, a 10-dof paediatric exoskeleton. Proceedings of the 19th International Conference on CLAWAR 2016

- Daniel Sanz-Merodio, Manuel Cestari, Juan Carlos Arevalo and Elena Garcia. Implementation of an adjustable compliant knee joint in a lower-limb exoskeleton. 16th International Conference on Climbing and Walking Robots.Sidney, 2013. (Winner of conference best paper)

- Daniel Sanz-Merodio, Manuel Cestari, Juan Carlos Arevalo, Elena Garcia. Gait parameter adaptation for lower-limb exoskeletons. International WorkConference on Bioinformatics and Biomedical Engineering, Granada, Spain 2013: $667-675$

- Daniel Sanz-Merodio, Manuel Cestari, Juan Carlos Arevalo, Xavier Carrillo, E. Garcia. Development of a Lower-Limb Active Orthosis and a Walker for Gait Assistance. ROBOT (1) 2013: 219-233

- Daniel Sanz-Merodio,Manuel Cestari, Juan Carlos Arevalo and Elena Garcia. A lower-limbexoskeleton for gait assistance in quadriplegia. IEEE International Conference on Robotics and Biomimetics (ROBIO) Guangzhou, China, 2012. (Best Paper in Robotics Finalist) 
- Daniel Sanz-Merodio, M. Cestari, J.C. Arevalo and E. Garcia. Taking Advantage of the Synergy for the Actuation in a Lower-Limb Active Orthosis. 15th International Conference on Climbing and Walking Robots.Baltimore, Maryland, 2012. . (Highly Commended - Innovation Award 2012).

- Daniel Sanz-Merodio, M. Cestari, J.C. Arevalo and E. Garcia. Strategies to reduce energy expenditure in the motion control of lower-limb orthoses. 15th International Conference on Climbing and Walking Robots.Baltimore, Maryland, 2012

- Manuel Cestari, Daniel Sanz-Merodio, Juan Carlos Arevalo, Xavier Carrillo and Elena Garcia. Actuator with adjustable-rigidity and embedded sensor for an active orthosis knee joint. 16th International Conference on Climbing and Walking Robots.Sidney, 2013. (Winner of conference best paper)

- J. C. Arevalo, M. Cestari, Daniel Sanz-Merodio and E. Garcia. Event driven ground-impedance identification for legged robots. 16th International Conference on Climbing and Walking Robots.Sidney, 2013. (Highly Commended - Best Technical Paper Award)

- Elena Garcia, Manuel Ocaña, Luis Miguel Bergasa, Manuel Ferre, Mohamed Abderrahim, Juan Carlos Arevalo, Daniel Sanz-Merodio, Eduardo J. Molinos, Noelia Hernandez, Angel Llamazares, Francisco Suarez-Ruiz, Silvia Rodriguez. Competing in the DARPA Virtual Robotics Challenge as the SARBOT Team. ROBOT (2) 2013: 381-396

- Juan Carlos Arevalo, Daniel Sanz-Merodio, Elena Garcia. Reactive Humanoid Walking Algorithm for Occluded Terrain. ROBOT (2) 2013: 397 410

- Juan Carlos Arevalo, Manuel Cestari, Gustavo Muñoz, Daniel Sanz-Merodio and Elena Garcia. Impedance Control for a Bioinspired Underactuated Leg, 15th International Conference on Climbing and Walking Robots.Baltimore, Maryland, 2012. (Highly Commended - Best Technical Paper Award) 
- Juan Carlos Arevalo, Xavier Carrillo, Manuel Cestari, Daniel Sanz-Merodio, and Elena Garcia. System Identification Applied to Contact Modeling: An ExperimentalInvestigation IEEE International Conference on Robotics and Automation. Karlsruhe, Germany, 2013.

- Juan Carlos Arevalo, Daniel Sanz-Merodio, Manuel Cestari and Elena Garcia. Parameterized Inverted and Double Pendulum Model for Controlling Lower-limb Active Orthosis IEEE International Conference on Robotics and Biomimetics (ROBIO) Guangzhou, China, 2012.

- Gustavo Muñoz, Manuel Cestari, Juan Carlos Arevalo, Daniel Sanz-Merodio and Elena Garcia. Benefits and Downwards of MRF Knee Damping in Legged Robots, 15th International Conference on Climbing and Walking Robots. Baltimore, Maryland, 2012. (Runner up - Best Technical Paper Award) .

- M. Cestari, D.Sanz-Merodio, J.C. Arevalo and E. Garcia. Development of Synergic Multiarticular Actuation in a Lower-limb Active Orthosis 13th Mechatronics Forum International Conference. 2012.

- E. Garcia, Daniel Sanz-Merodio, F. Sanchez, J.C. Arevalo and P. Gonzalez de Santos, Development of the ATLAS lower-limb active orthosis, 14th Int. Conf. Climbing and Walking Robots and the Support Technologies for Mobile Machines, Field Robotics ISBN: 13 978-981-4374-27-9 Paris, France, 2011.

\subsection{Technology transfer of thesis results}

\subsubsection{Patents}

The technical contributions of this thesis have been protected by three patents. They are currently under commercial exploitation by Marsi-Bionics S.L and have been extended internationally. 
- ES201431763 . E. Garcia, M. Cestari, D.Sanz-Merodio, and X. Carrillo "Exoskeleton for assisting human movement" (11-25-2015) Assignee: CSICUPM (85\%), M-B (15\%) PCT/ES2015/070855 (19.02.2016); US 20170340504A1; EU sol. 15862885.

- ES201330882. M. Cestari, D.Sanz-Merodio, y E. Garcia "Articulation with controllable stiffness and force-measuring device" (06-13-2013). Licensed to M-B by CSIC and UPM. European Patent (3009240) Conceded 6/11/2017; WO2014198979.

- ES201330882. M. Cestari, D.Sanz-Merodio, J.C. Arevalo y E. Garcia. "Walker comprising mechanism for assisting a user in standing and sitting operations" (10-11-2012). Licensed to M-B by the CSIC and UPM. Extended: EP2907495; US2015265490; WO2014057153.

The above listed patents have been transfered and contributed to the creation of Marsi Bionics technology-based company, spin-off from CSIC-UPM.

Based on this thesis results, two evolutions of pediatric exoskeletons, the ATLAS 2020 and the ATLAS 2030, have been developed, see Figure 8.1, which are in the process of commercialization. With these exoskeletons, clinical tests have been performed in two Spanish hospitals with very positive results: Sant Joan de Déu children Hospital and Ramón y Cajal University Hospital.

The commercial ATLAS 2030 exoskeleton is currently in use in the rehabilitation Service of Sant Joan de Déu Children Hospital for the regular therapy of children affected by Spinal Muscular Atrophy.

This $\mathrm{PhD}$ thesis has been performed under the frame of the following reseach projects:

- ATLAS project: Performance and control of power amplification and mobility devices in humans and robots(MICINN DPI2010-18702)

- KINDER project: Exoskeletons as an instrument to improve the quality of life of children with spinal muscular atrophy and cerebral palsy (MINECO DPI2013-40504-R) 


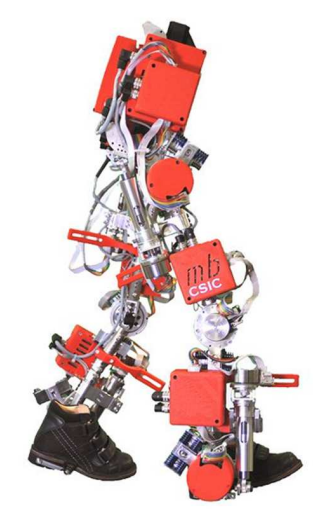

(a)

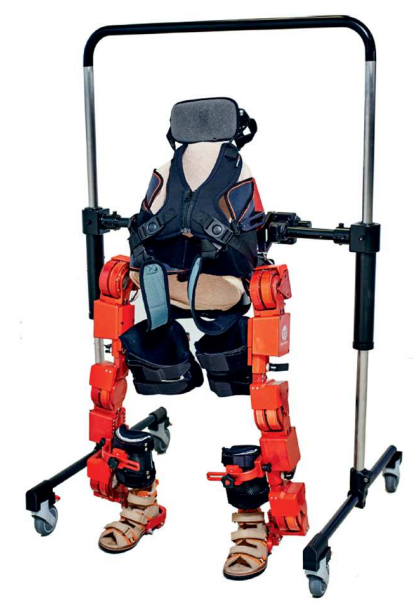

(b)

Figure 8.1: ATLAS 2020 prototype (a) and the ATLAS 2030 commercial(b) exoskeletons

- EXOTrainer project: Clinical Evaluation of Gait Training with Exoskeleton in Children with Spinal Muscular Atrophy (European Commision - Echord++ Experiment 401) 

A man's life is an appendix to his

heart.

Rober South

APPENDIX

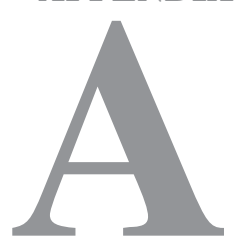

\section{ATLAS active orthosis}

The ATLAS exoskeleton can be classified as an active orthosis because it is devised for gait assistance, not for load-carrying augmentation[116]. In orthotic terminology, it can be considered to be an active THKAFO (trunk-hip-knee-anklefoot orthosis). ATLAS prototype is designed to support a $25-\mathrm{kg}$ child and assist the child with ambulation at a moderate speed $(\leq 0,6 \mathrm{~m} / \mathrm{s})$.

The ATLAS exoskeleton is considered to be a lightweight, easily handled and simple-to-don robotic system. It consists of the following primary components (Figure A.1):

1. Mechanical structure: As a lower limb orthosis, it must be simple, lightweight, strong, durable and cosmetically acceptable. It is a 6-DOF mechanism, with 3 DOF per leg (the hip, knee and ankle) to enable the user to move in the sagittal plane. The structure is attached to the user's body with comfortable belts. Because the exoskeleton is worn under the user's clothing, it must not be bulky.

2. Actuation system: The flexion and extension motion of the hip and knee joints is driven by electric brushless Maxon motors combined with Harmonic Drive units. This setup provides a repeated peak torque of up to $57 \mathrm{Nm}$ and 


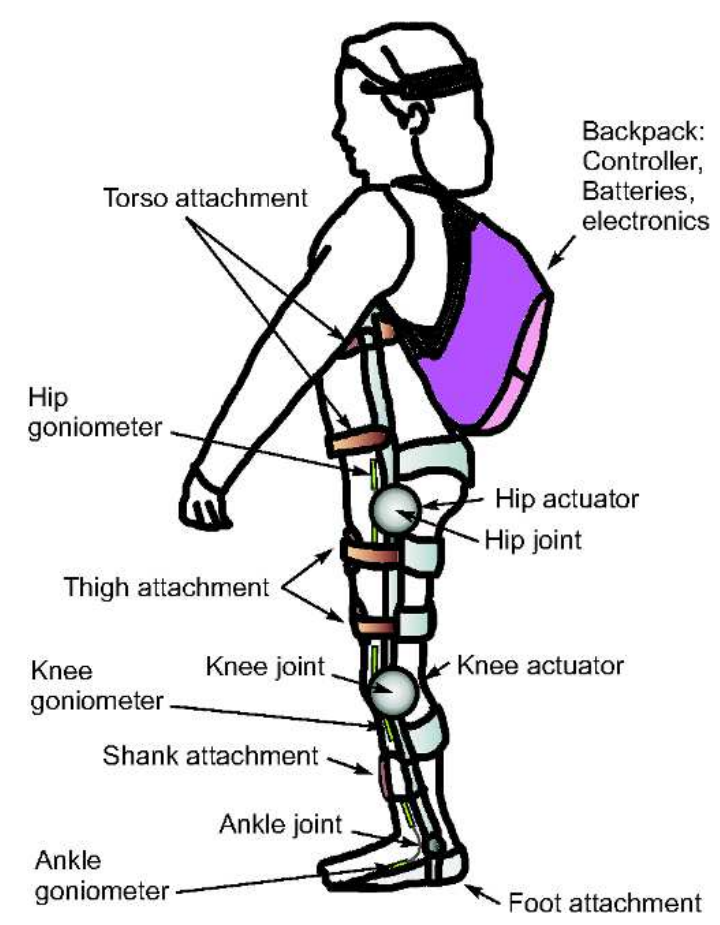

Figure A.1: Concept of the ATLAS exoskeleton showing main components

an average torque of $32 \mathrm{Nm}$ at a speed higher than $20 \mathrm{rpm}$ for each actuated joint. The equipment consists of a motor-gearbox set that provides a large power-to-weight ratio while remaining flat enough not to disturb the user motion.

3. Sensorial system: The sensorial system consists of goniometers at the user's hip, knee and ankle, which measure joint angles, and an in-shoe plantar pressure measurement system on each foot, which provide the centre of pressure $(\mathrm{COP})$ trajectory.

4. Motor controller: Individual control of each of the motors is provided by Maxon EPOS controllers that can provide position, velocity or current control. All of the EPOS are connected by a CANopen bus to the main controller, which is based on a National Instruments SingleBoard RIO, with a real-time operating system and FPGA. An impedance controller is programmed to follow parameterised joint trajectories while reacting compliantly to small perturbations in the sagittal plane. The controller, related electronics, sensor 
amplifiers and batteries are placed inside a backpack. The backpack weight is 4.3 kilos.

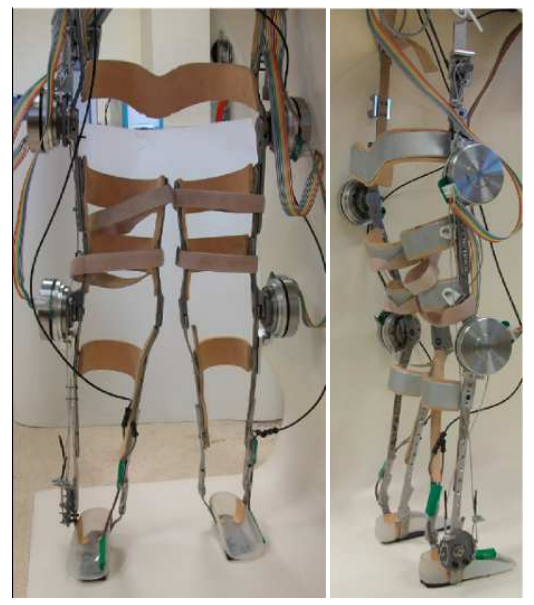

Figure A.2: Front and back views of ATLAS prototype.

\section{A.1 Kinematic analysis}

ATLAS balance is ensured making use of a walker. Since the walker (See Appendix B) dynamically independent both legs, the kinematics and dynamics is calculated for each leg separately. The mathematical model of the leg is considered as a threelink planar manipulator with actuated joints.

\section{A.1.1 Direct kinematics}

From the angles of the joints of the exoskeleton, through direct kinematics, the position and orientation of the end effector of the robot is obtained, that is, the foot. Figure A.4 shows a schematic of the model to be used in kinematics.

Taking the hip joint as the origin of the reference system, the position of the knee is calculated with the equation:

$$
\begin{aligned}
& x_{1}=L_{\text {thigh }} \sin \theta_{1} \\
& y_{2}=L_{\text {shank }} \cos \theta_{1}
\end{aligned}
$$




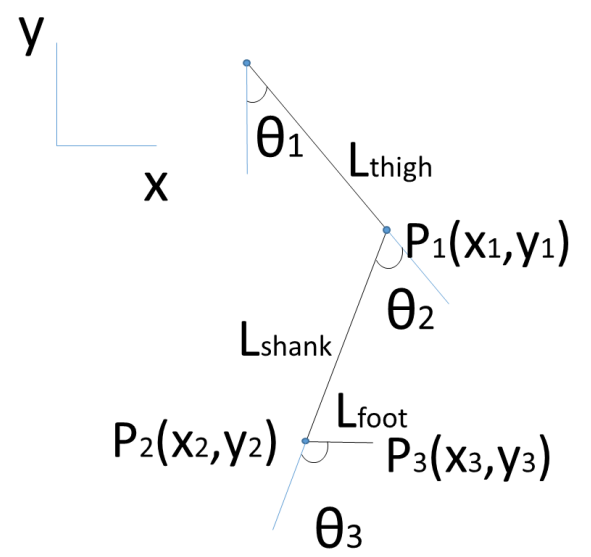

Figure A.3: Scheme with the main angles of the leg.

Similarly, the position of the ankle with respect to the reference system, taking the angle of the knee as negative:

$$
\begin{aligned}
& x_{2}=x_{1}+L_{\text {shank }} \sin \theta_{12} \\
& y_{2}=y_{1}+L_{\text {shank }} \cos \theta_{12}
\end{aligned}
$$

Where the convention has been applied:

$$
\begin{aligned}
& \cos \theta_{12}=\cos \left(\theta_{1}+\theta_{2}\right) \\
& \sin \theta_{12}=\sin \left(\theta_{1}+\theta_{2}\right)
\end{aligned}
$$

Also the position of the tip of the foot is related as:

$$
\begin{aligned}
& x_{3}=x_{2}+L_{\text {foot }} \sin \theta_{123} \\
& y_{3}=y_{2}+L_{\text {foot }} \cos \theta_{123}
\end{aligned}
$$

\section{A.1.2 Inverse kinematics}

To place the foot in a certain point with a given orientation, it is necessary to establish certain angles in each of the joints. This is calculated with the inverse kinematics formulation. 


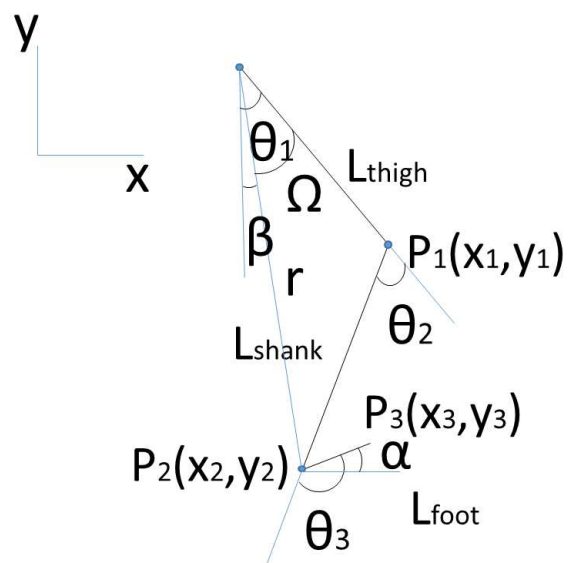

Figure A.4: Scheme for the calculation of inverse kinematics.

From Figure A.4, Equations A.9 and A.10 are obtained.

$$
\begin{gathered}
\beta=\operatorname{atan}\left(\frac{x_{2}}{y_{2}}\right) \\
L_{\text {shank }}^{2}=L_{\text {tight }}^{2}+r^{2}+2 L_{\text {tight }} r \cos (\Omega)
\end{gathered}
$$

where $r^{2}=x_{2}^{2}-y_{2}^{2}$.

From these equations $\Omega$ is obtained:

$$
\Omega=\operatorname{acos}\left(\frac{L_{\text {shank }}^{2}-L_{\text {tight }}^{2}-r^{2}}{-2 L_{\text {tight }} r}\right)
$$

The angle of the hip is:

$$
\theta_{1}=\Omega+\beta
$$

From the cosine theorem the angle of the knee is:

$$
\theta_{2}=\pi-\operatorname{acos}\left(\frac{r^{2}-L_{\text {tight }}^{2}-L_{\text {shank }}^{2}}{2 L_{\text {tight }} L_{\text {shank }}}\right)
$$

The ankle angle is placed according to the ground inclination $\alpha$ :

$$
\theta_{3}=\theta_{2}-\theta_{1}+\frac{\pi}{2}+\alpha
$$




\section{A.2 Dynamic model of the active orthosis ATLAS}

The ATLAS orthosis calculates parameterized trajectories for each terrain and gait characteristic. To calculate the kinetic and potential energy at each instant for the parameterized paths, a three-link planar mode will be used. In this subsection, the equations that are used the control system are presented.

Since a walker is used for walking stability that makes each leg independent, a modeling the dynamics of each leg independently is considering. So, the control will treat a three-link planar manipulator confined to the vertical plane (see Figure A.5). The motion of the manipulator is driven by three motors in each joint: hip, knee, and ankle. To facilitate the notation of the equations, the hip is named as joint 1 , the knee as joint 2 and the ankle as joint 3 .
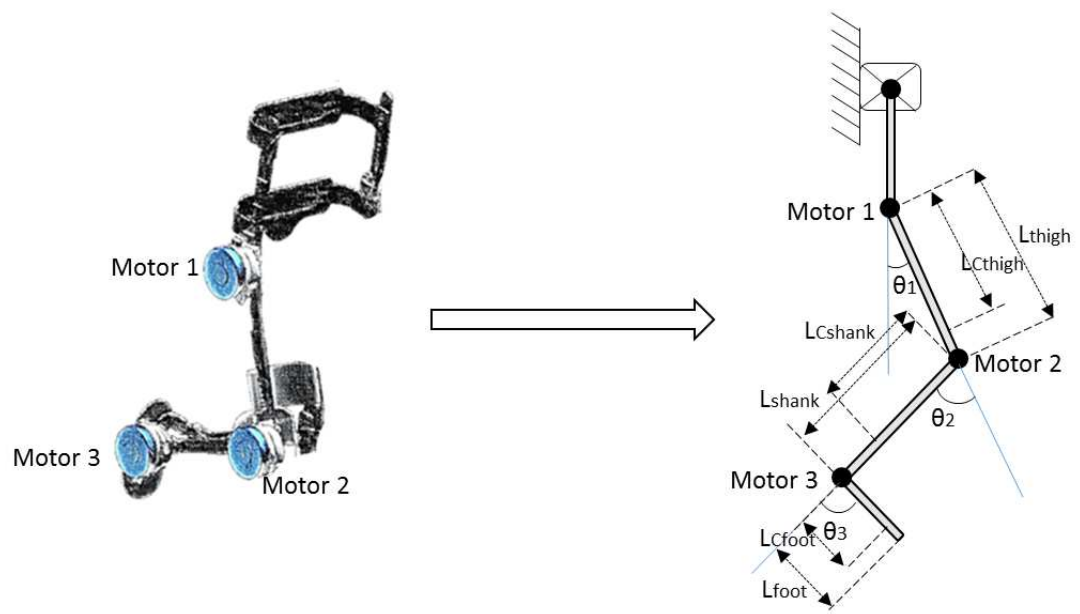

Figure A.5: Three-link planar manipulator

To obtain the dynamic model of the system the Lagrangian formulation is used, which establishes the equations:

$$
\begin{gathered}
\frac{d}{d t} \frac{\partial \boldsymbol{L}}{\partial \dot{q}_{i}}-\frac{\partial \boldsymbol{L}}{\partial q_{i}}=\tau_{i} \\
\boldsymbol{L}=\boldsymbol{K}-\mathbf{U}
\end{gathered}
$$


where $\theta_{i}$ are the joint coordinates, $\tau_{i}$ is the vector of torques applied to the joints, $\boldsymbol{L}$ is the Lagrangian function, $\boldsymbol{K}$ is the kinetic energy and $\mathbf{U}$ is the potential energy.

Calling $l_{i}$ to the length of each link, and $l_{C i}$ to the locatization of the center of mass, $i=1,2,3$.

Denoting by $m_{i}$ the mass of each link and $m_{R i}$ the mass of each actuator. With this notation, the Lagrangian function of the manipulator's motion is:

$$
\begin{gathered}
\boldsymbol{L}=\frac{1}{2} A_{11}{\dot{\theta_{1}}}^{2}+\frac{1}{2} A_{22}{\dot{\theta_{2}}}^{2}+\frac{1}{2} A_{33}{\dot{\theta_{3}}}^{2}+ \\
A_{12} \dot{\theta_{1}} \dot{\theta_{2}} \cos \left(\theta_{2}-\theta_{1}\right)+A_{13} \dot{\theta_{1}} \dot{\theta_{3}} \cos \left(\theta_{3}-\theta_{1}\right)+A_{23} \dot{\theta_{2}} \dot{\theta_{3}} \cos \left(\theta_{3}-\theta_{2}\right) \\
-\left[V_{01} g \sin \theta_{1}+V_{02} g \sin \theta_{2}+V_{03} g \sin \theta_{3}\right]
\end{gathered}
$$

where

$$
\begin{gathered}
A_{11}=m_{1} l_{C 1}^{2}+I_{1} *\left(m_{2}+m_{R 2}+m_{3}+m_{R 3}\right) l_{1}^{2} \\
A_{22}=m_{2} l_{C 2}^{2}+I_{2} *\left(m_{3}+m_{R 3}\right) l_{2}^{2} \\
A_{33}=m_{3} l_{C 3}^{2}+I_{3} \\
A_{12}=m_{2} l_{1} l_{C 2}+\left(m_{3}+m_{R 3}\right) l_{1} l_{2} \\
A_{13}=m_{3} l_{1} l_{C 3} \\
A_{23}=m_{3} l_{2} l_{C 3} \\
V_{01}=m_{1} l_{C 1}+\left(m_{2}+m_{R 2}+m_{3}+m_{R 3}\right) l_{1} \\
V_{02}=m_{2} l_{C 2}+\left(m_{3}+m_{R 3}\right) l_{2} \\
V_{03}=m_{3} l_{C 3}
\end{gathered}
$$

The above equation includes the kinetic and potential energy of the robot at all times. This calculation of the potential and kinetic energy of the robot is carried out in each position and speed of the robot to determine its maximum and thus exploit the passive dynamics by means of the variation of impedance of the robot.

The equation of the dynamics of the system is obtained from the equations A.15, A.16 and A.17 


$$
\boldsymbol{M}(\boldsymbol{q}) \frac{d^{2} \boldsymbol{q}}{d t^{2}}+\boldsymbol{H}(\dot{\boldsymbol{q}}, \boldsymbol{q})=\boldsymbol{\tau}
$$

where $\boldsymbol{\tau}=\left(\tau_{1}, \tau_{2}, \tau_{3}\right)^{T}$

$$
\begin{gathered}
m_{11}=A_{11}, m_{12}=A_{12} \cos \left(\theta_{2}-\theta_{1}\right), m_{13}=A_{13} \cos \left(\theta_{3}-\theta_{1}\right), \\
m_{21}=m_{12}, m_{22}=A_{22}, m_{23}=A_{23} \cos \left(\theta_{3}-\theta_{2}\right), \\
m_{31}=m_{13}, m_{32}=m_{23}, m_{33}=A_{33} \\
h_{1}=-A_{12}{\dot{\theta_{2}}}^{2} \sin \left(\theta_{2}-\theta_{1}\right)-A_{13} \dot{\theta}_{3}^{2} \sin \left(\theta_{3}-\theta_{1}\right)+V_{01} g \cos \theta_{1}, \\
h_{2}=A_{12} \dot{\theta}_{1}^{2} \sin \left(\theta_{2}-\theta_{1}\right)-{A_{23} \dot{\theta}_{3}}^{2} \sin \left(\theta_{3}-\theta_{2}\right)+V_{02} g \cos \theta_{2}, \\
h_{3}=A_{13} \dot{\theta}_{1}^{2} \sin \left(\theta_{3}-\theta_{1}\right)+A_{23} \dot{\theta}_{2}^{2} \sin \left(\theta_{3}-\theta_{2}\right)+V_{03} g \cos \theta_{3}
\end{gathered}
$$

According to this model and given the above equations, the position of the leg end effector in a position and at a bounded speed, it setting by following this control law[120]:

$$
\tau=M(q) v+H(\dot{q}, q)
$$

where $\boldsymbol{v}=\left(v_{1}, v_{2}, v_{3}\right)^{T}$

Then for cases where $\boldsymbol{M}(\boldsymbol{q}) \neq 0$ the following is fulfilled:

$$
v_{1}=\frac{d^{2} \theta_{1}}{d t^{2}}, v_{2}=\frac{d^{2} \theta_{2}}{d t^{2}}, v_{3}=\frac{d^{2} \theta_{3}}{d t^{2}}
$$

Therefore, according to this law of inverse dynamics control, the system is controlled providing adequate acceleration to each of the joints.

\section{A.3 Mechanical configuration}

The mechanical structure of the ATLAS exoskeleton is custom made for the user by a specialised orthotic manufacturer to ensure user comfort. The manufacturing material is mostly duraluminium with stainless steel at the joints. The exoskeleton 
is a lightweight device $(6.5 \mathrm{~kg})$, which facilitates its handling, e.g., donning and removal. The structure is tightly attached to the user using 2 attachments at the torso, 2 attachments at the thigh and one attachment at the shank. The attachments of the mechanical structure to the body should not hurt the user under any circumstances (and even if the user does not feel pain, the skin and tissue should not be damaged). The attachments include pressure control, flexible material, and tissue tolerance against compression and shear force. The user's foot fits in a thermoplastic molded UCBL shoe insert [121]. While correcting and holding the heel in a neutral position, the UCBL also controls the inside arch of the foot and the outside edge of the forefoot. These three corrective forces hold the foot in a neutral position[121]. Figure A.2 shows different views of the mechanical structure.

This first prototype of the ATLAS exoskeleton provides motion only in the sagittal plane using two actuated DOF per leg at the hip and the knee and a passive ankle. It is widely known that the most relevant torques involved in human ambulation occur at the sagittal joints. Thus, this first approximation suffices for preliminary experiments. Safety dictates that the range of motion of the exoskeleton joints should not be larger than the range of motion of the user's joints. Additionally, the range of joint motion during ambulation should resemble the average human walking ranges of motion. Table A.1 lists the ATLAS joint mechanical limits and compares them with human joint limits (based on Hamilton et al. [122]).

Table A.1: ATLAS joint limits compared with average human joint range of motion (angles in degrees).

\begin{tabular}{lccc}
\hline & $\begin{array}{c}\text { Human } \\
\text { maximum } \\
(\mathrm{deg})\end{array}$ & $\begin{array}{c}\text { ATLAS } \\
\text { maximum } \\
(\mathrm{deg})\end{array}$ & $\begin{array}{c}\text { Walking } \\
\text { maximum } \\
(\mathrm{deg})\end{array}$ \\
\hline Hip flexion & 110 & 90 & 32 \\
Hip extension & 30 & 10 & 10 \\
Knee flexion & 120 & 90 & 63 \\
Ankle dorsiflexion & 20 & 18 & 14 \\
(Flexion) & & & \\
$\begin{array}{l}\text { Ankle plantar flexion } \\
\text { (Extension) }\end{array}$ & 50 & 20 & 20 \\
\hline
\end{tabular}


The ATLAS exoskeleton can be covered by the user's clothing and shoes and is nearly unnoticeable. The metal uprights are extendible and adjust each limb length to the user's limbs as the user matures and grows. The thigh length can be adapted from 270 to 460 and the shank length can be changed from 250 to $390 \mathrm{~cm}$. Even the width of torso can be adapted from 230 to $320 \mathrm{~cm}$. So, this mechanical structure will be valid during four years.

\section{A.4 Electrical architecture and sensorial system}

The electrical architecture of the ATLAS exoskeleton has been divided into three independent and interconnected subsystems: high-level control subsystem, low level control subsystem and the power subsystem that distributes the necessary power to the various subsystems.

The high-level control subsystem is based on a National Instruments board (MyRIO) which features a real-time processor and FPGA Xilinx. Based on the information from the sensors and the intention and commands of the user, this highlevel controller is in charge of generating trajectories, angles and speeds required for each joint and send them through an I2C serial line to the local drivers. This module receives information from WIFI connection of user software running on a PC or tablet.

The low level control subsystem is responsible for receiving the speed command of each joint (by a I2C protocol) and sending these signals to the motor windings. This module comprises a microcontroller ATMEL, a power driver and the electronics to the acquisition of sensors. Each of these modules is able to command fully two joints. All the sensors in the orthosis are received by this low level control system. These signals are then sent to the master through the serial line for further processing.

The main sensorial data that are received in each joint are acquired by the following sensor systems:

- Position sensor of the joint $\theta_{\text {joint }}$, measured by a magnetic encoder.

- Encoder of the joint motor. 
- Force applied in the joint.

- Pressure sensors at the insoles. These sensors are only sent to the control subsystem that moves the ankles. Each foot features a 32 resistive pressure sensors distributed throughout the shoe insole, which sends information about the center of pressure of the foot and the weight distribution.

- Inertial magnetic unit (IMU) in the pelvis, to ensure the right posture of the user. This sensor goes directly to the master unit.

The power subsystem includes batteries, a pack of Litio-Ion 24 Vcc battery, and theirs control (load and overall status) and circuitry needed to generate the auxiliary voltages necessary to power other electronics. A distributed configuration with no power wiring between the two legs facilitates placement of the exoskeleton by separating two limbs.

\section{A.5 Gait trajectory generation}

From the position of the joint, the pressure on the insoles and the IMU information, the master generates an array of angles for each joint. The basic parameters to generate the arrays of positions are:

- Step length. distance between one successive placement of the same foot.

- Height of step. Lifting height of the foot when walking

- Gait cycle speed.

- Length of each of the links of the exoskeleton. (Length femur, tibia length, foot length). From these lengths the controller calculates the direct and inverse kinematics, for correct positioning in the Cartesian plane.

- Standing up and sitting down speed.

A cubic spline is made from the trajectory obtained starting from these specifications and reference positions and speeds for each joint are obtained. A PID 


\section{A. ATLAS ACTIVE ORTHOSIS}

controller is configured with these position and speed references and setpoints giving references to control the speed motor drivers. The transition from the standby position to walk and vice versa is made by generating a path between two points and making again a spline that generates intermediate position and velocity references.

Figure A.6 shows how the foot trajectory changes when body height and step length change.

These new reference trajectories must be defined without abrupt movement. That is, the final path that the foot must follow must be differentiable. To achieve the original CGA motion in the foot, the angle and angular speed references at the knee are as shown in Figure A.7 (a). Both angle and angular speed references are differentiable. When the reference path is changed to a path with more ground clearance (see Figure A.6 (a)), the angular speed reference is not differentiable. Figure A.7(b) shows abrupt speed changes.

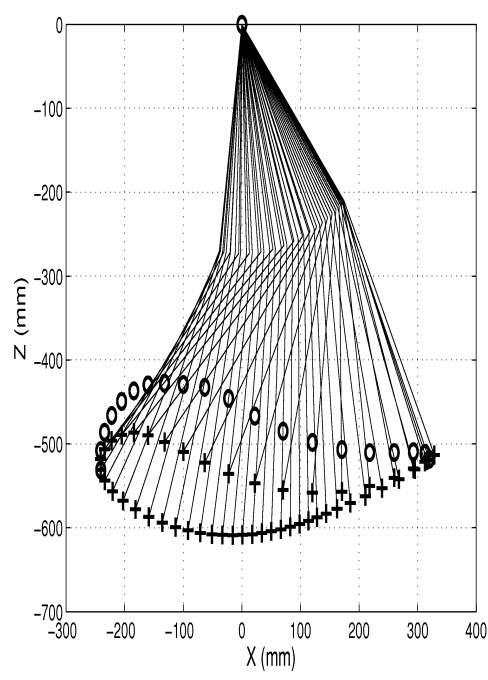

(a)

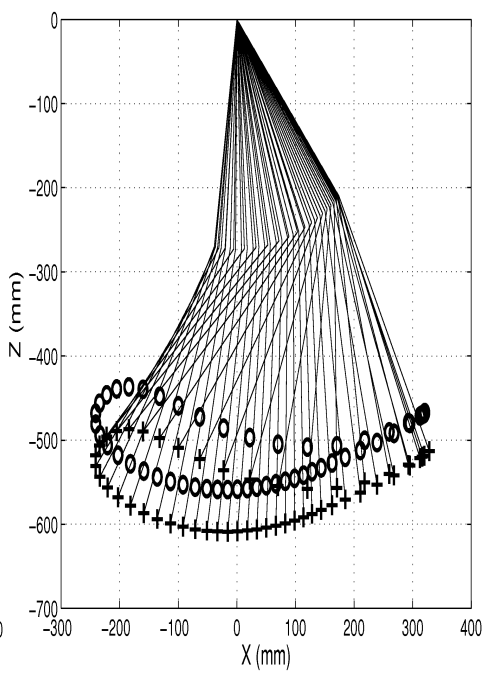

(b)

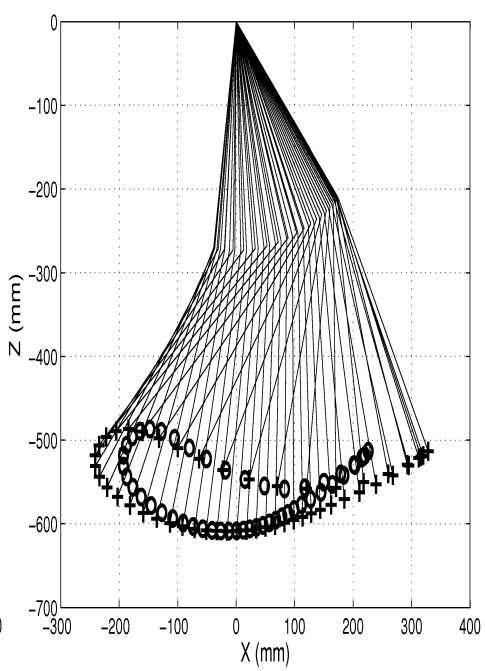

(c)

Figure A.6: Original foot path obtained by CGA $($ marker + ) and modified (marker $o$ ) to (a) provide increased ground clearance, (b) reduce body height and (c) reduce step length.

To avoid these discontinuities, which would produce a tremor in the limb, the transitions between the two trajectories have been modulated by a unitary Gaussian 
function:

$$
F_{\mu, \sigma}\left(X_{d e s}\right)=\frac{1}{\sigma \sqrt{2 \pi}} e^{\frac{\left(X_{d e s}-\mu\right)^{2}}{2 \sigma^{2}}},
$$

where $\mu$ is the mean and $\sigma$ is the standard deviation of the Gaussian function. To centre the Gaussian curve correctly, $\mu=0$ was selected, and $\sigma=\frac{1}{\sqrt{2 \pi}}$ so that the amplitude of the Gaussian is unitary and adequate bonding of the curves is obtained. Typically, these windows are used in communication filtered processes. Figure A.7 (c) shows the knee references when the trajectories are jointly modulated by a unitary Gaussian window, and differentiable references are obtained . Thus, a smoother trajectory is obtained, which is more comfortable for the user.

While walking the current, the strength and the temperature of the engine are monitored. Two types of error setpoints are establish in the case of temperature and current, the critical and the problematic. Critical alarm would stop the exoskeleton and the problematic alarm would give a warning for a possible review of the state of the actuator.

With the sensing of force, a upper limit is established to avoid damaging the user. If with the restriction of this limit the exoskeleton could not walk properly, that is, if the tracking error was bigger a margin for a while, an emergency procedure will be initiated.

\section{A.6 Control scheme}

From the parametric trajectories obtained and the sensory information, a control scheme has been developed and implemented, see Figure A.8. For each joint the desired position $\theta_{\text {des }}$ is obtained by the inverse kinetics with which the feedback loop is closed and the velocity $\dot{\theta}_{\text {des }}$ that is introduced as feed-forward.

The force at each joint is calculated from compliance controller that was adapted from [123]. This controller uses parameterised joint trajectories as equilibrium points. Small forces are allowed to separate slightly from the equilibrium points to facilitate compliance.

The locomotion controller determines desired foot forces. The actual cartesian force vector is calculated from the values obtained with the insoles. The force 


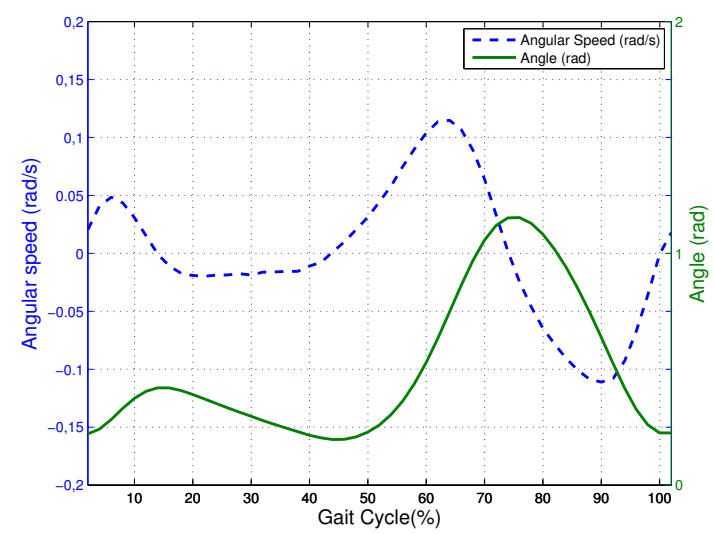

(a)

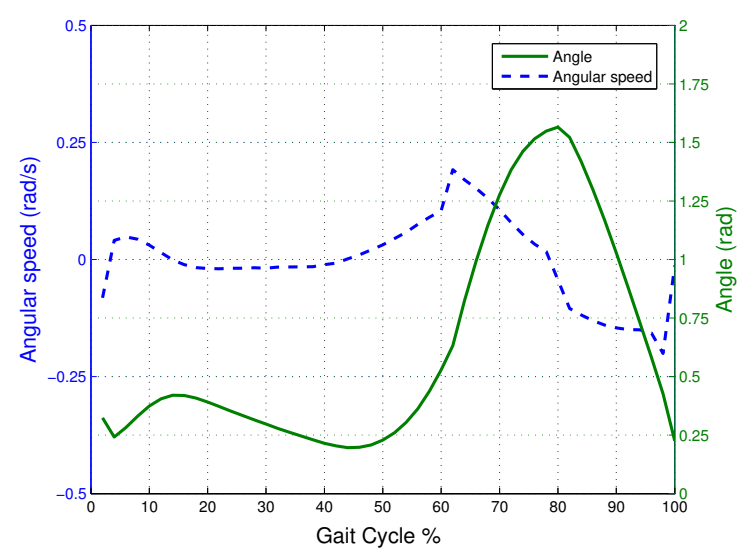

(b)

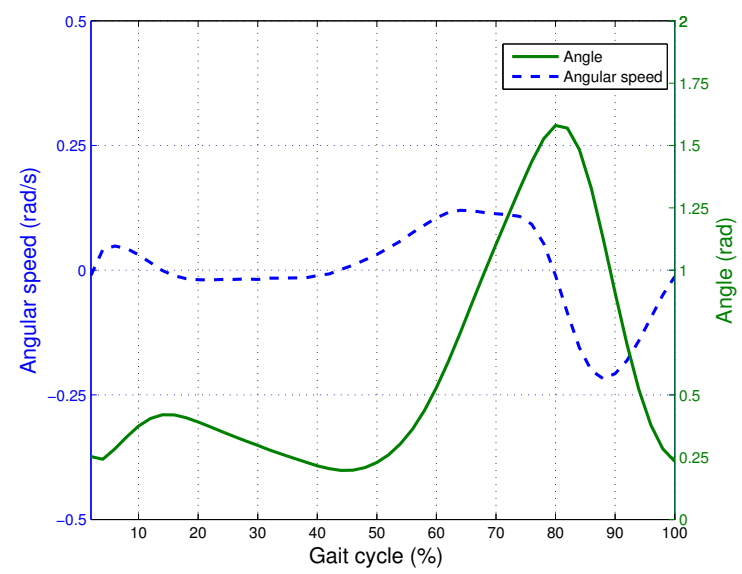

(c)

Figure A.7: Smoothing position and velocity curves in the modification of gait. (a) Angles and speeds at the knee for the original CGA path. (b) Angles and speeds at the knee when the gait is modified to provide larger ground clearance without using Gaussian windows and (c) using Gaussian windows. 
components are obtained with a least-squares inverse dynamics approach [? ]. Therefore, the active compliance equation for each leg has the following form:

$$
F_{d e s}-F=-K_{p}\left(x_{d e s}-x\right) K_{f}^{-1}+X^{r e f} K_{f}^{-1}
$$

where $x$ is the vector actual position of the foot in swing or the hip in stance, $x_{d e s}$ is the vector of reference positions obtained from parameterized trajectories, $F$ is actual force vector from joint position in swing or from insoles during stance and $F_{d e s}$ is the vector of the desired Cartesian foot forces, which are obtained using the force-distribution algorithm [124]. $K_{p}$ and $K_{f}$ are diagonal matrices of gains. $X^{r e f}$ are position references as inputs to joint controllers after inverse kinematics transform.

With this controller, the actual joint positions commanded to the inner controller represent a trade-off between user joint angle errors and force errors at the foot. Equation A.22 corresponds to the equation of motion of a spring system, being $K_{p} K_{f}^{-1}$ an effective stiffness of the system.

To calculate the torque that each joint must do to achieve the force $F_{\text {des }}$ in the Cartesian plane uses the Jacobian matrix:

$$
\tau_{d e s}=J^{T} F_{\text {des }}
$$

With the the desired position $\theta_{\text {des }}$ the desired velocity $\dot{\theta}_{F F}$ and torque $\tau_{d e s}$, the desired joint speed $\dot{\theta}_{d e s}$ is obtained. This speed is sent to the ESCON motor controller. This controller have been previously tuned for the calculation of the parameters of Figure A.8.

The position of the joint $\theta_{\text {joint }}$, is measured by a magnetic encoder and the torque performed by the joint is obtained as:

$$
\tau=K_{\text {sea }} \sigma_{\text {spring }}
$$

with these components the control loop of all variables is closed. 


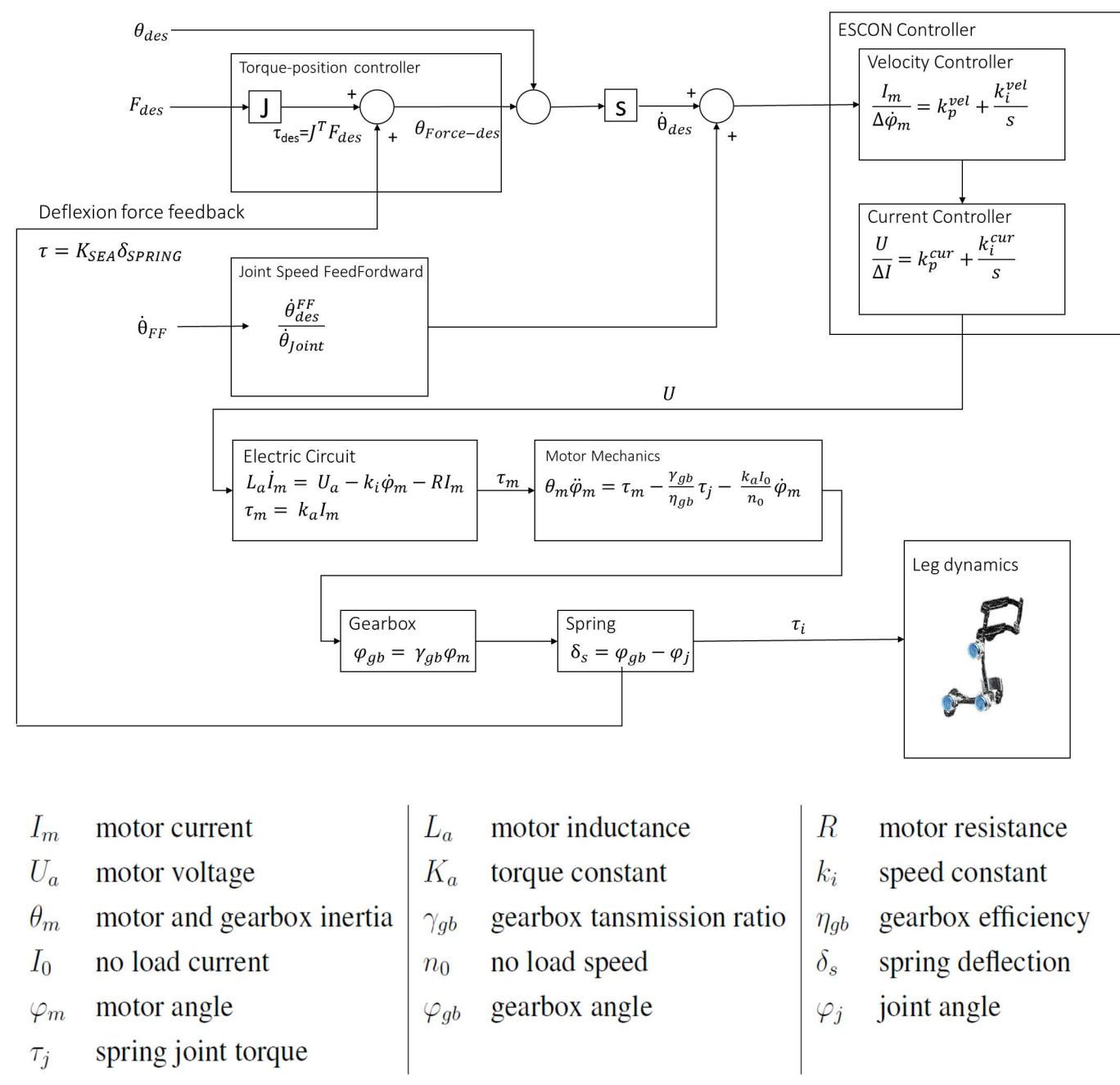

Figure A.8: General control scheme of the active orthosis. 
This end is just a new begging.

J. Stanford

APPENDIX

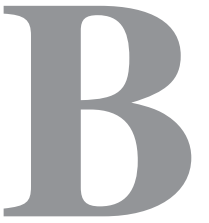

\section{Walker concept and design}

The development of fully functional active orthoses for paralysed patients requires further research. Specifically, the development of active orthoses for completely paralysed patients, such as quadriplegics, has not been considered because of the added difficulty of stability control. In the development of ATLAS project these objectives are tackled through the development of a walker and an active orthosis, providing the user the ability to stand, sit and walk. The walker has been designed to provide stability in lateral and frontal planes. This is a height adjustable walker, with smart features to assist the user allowing them to sit down and stand up while still attached to the walker. It is intended for the development of daily activities and ensuring adequate stability for users to submit greater mobility limitations.

The walkers used for rehabilitative function are not designed for its incorporation and use in an user's everyday activities. Those devices which incorporate mechanisms to enable sitting, standing or exercise muscle toning are characterized by large structures or rigid configuration systems that do not allow users' requirements in a regular social environment. The patent document presents a practical device that provides the ability to sit and stand, but this system requires a movement of the wheels of the walker on the horizontal plane to compensate for the natural movement when sitting. Similarly, in case of locking the wheels of the 


\section{B. WALKER CONCEPT AND DESIGN}

device, this restriction of movement causes an unnatural movement of the user's body. For these reasons, we have designed an adjustable height walker with an integrated intelligent control system that provides greater security. The kinematic chain which is derived from the connections to the user, provides two degrees of freedom to the sitting mode sufficient to fit the positions described in the sitting and standing user. Figure B.1 shows how the device adapts to the user's movements while sitting. Figure B.2 presents snapshots of a user in a test while sitting down, see how the walker allow to follow the body trajectory without displacement.
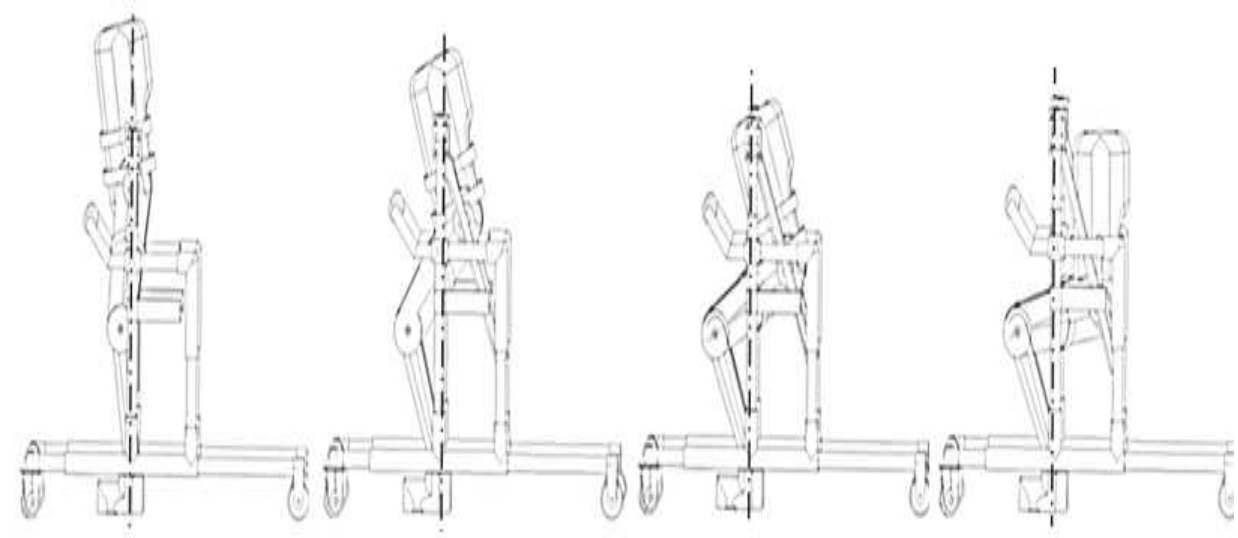

Figure B.1: The walker adapts to the user's movements while sitting.
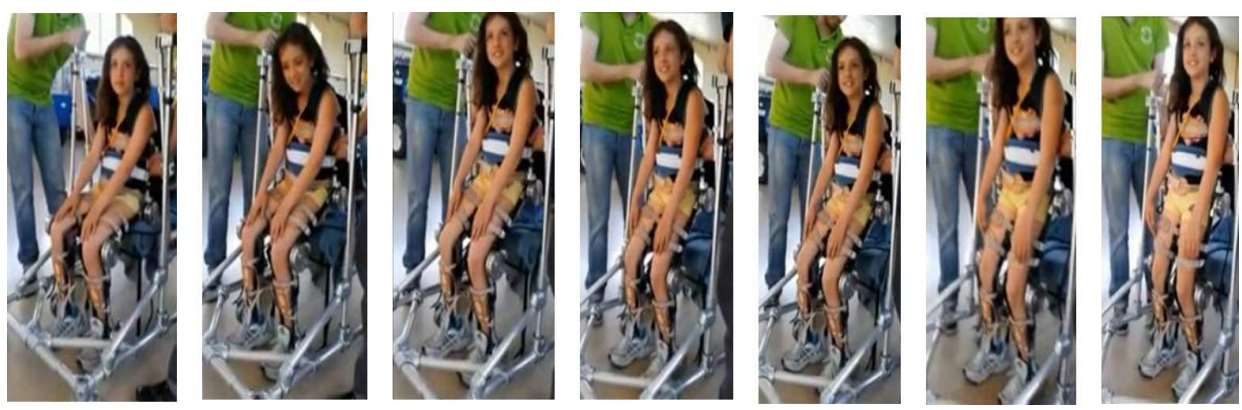

Figure B.2: Snapshots of a user in a test while sitting down.

These results in the walker design have led to a national patent number P201231567 entitle "Walker comprising mechanism for assisting a user in standing and sitting operations" PCT, EU, USA. The invention relates to a walker comprising a mechanism for assisting a user in standing and sitting operations, comprising a support- 
ing structure provided with movement means, a device for securing the user to the walker, and a system for blocking the movement means, where the supporting structure comprises at least one pivoting arm that guides the device for securing the user to the walker, a guide support that is fixed to the walker and guides the arm , and a control module that controls the system for blocking the movement means and comprises means for selecting a working mode of the walker, from "walking mode" and "sitting/standing mode".

A video of a performance with a dummy can be viewed online at http://www.youtube.com/watch? $v=B g n p C u C \operatorname{spN} U$. 



\section{Bibliography}

[1] Brian S. Armour, Elizabeth A. Courtney-Long, Michael H. Fox, Heidi Fredine, and Anthony Cahill. Prevalence and Causes of Paralysis-United States, 2013. American Journal of Public Health, 106(10):1855-1857, August 2016. xix, 2, 6

[2] Thomas A. McMahon. Mechanics of locomotion. The International Journal of Robotics Research, 3(2):4-28, 1984. Xix, 24

[3] Donald F. Hoyt and C. Richard Taylor. Gait and the energetics of locomotion in horses. Nature, 292(5820):239-240, 1981. xix, 23, 25, 26

[4] Arthur D. Kuo, J. Maxwell Donelan, and Andy Ruina. Energetic consequences of walking like an inverted pendulum: step-to-step transitions. Exercise and sport sciences reviews, 33(2):88-97, 2005. Xx, 27, 33

[5] Amy Silder, Bryan Heiderscheit, and Darryl G. Thelen. Active and passive contributions to joint kinetics during walking in older adults. Journal of biomechanics, 41(7):1520-1527, 2008. xx, 33, 34, 35

[6] J. J. Eng and D. A. Winter. Kinetic analysis of the lower limbs during walking: what information can be gained from a three-dimensional model? Journal of Biomechanics, 28(6):753-758, June 1995. Xx. 40, 41

[7] Y. C. Fung. Biomechanics: Mechanical Properties of Living Tissues. New York, NY ;Berlin u.a., 2 edition, June 1993. Xxi, 51,52 
[8] S. H Collins and A. D Kuo. Recycling energy to restore impaired ankle function during human walking. Plos One, 5(2):93-97, 2010. Xxiii, 89, 91. 100

[9] Gabriele Bovi, Marco Rabuffetti, Paolo Mazzoleni, and Maurizio Ferrarin. A multiple-task gait analysis approach: kinematic, kinetic and emg reference data for healthy young and adult subjects. Gait \& posture, 33(1):6-13, 2011. xxiii, xxiv, Xxix, 28, 112, 119, 121, 123

[10] Kate Wong. An ancestor to call our own. Scientific American, 13:4-13, 2003. 1

[11] Peter S. Rodman and Henry M. McHenry. Bioenergetics and the origin of hominid bipedalism. American Journal of Physical Anthropology, 52(1):103-106, 1980. 1

[12] World Health Organization. Fact sheet on wheelchairs. pages 1-4, 2010. 2

[13] Alan EH Emery. Population frequencies of inherited neuromuscular diseases - a world survey. Neuromuscular disorders, pages 19-29, 1991. 2

[14] G. T. Carter, R. T. Abresch, W. M. Fowler, E. R. Johnson, D. D. Kilmer, and C. M. McDonald. Profiles of neuromuscular diseases. Spinal muscular atrophy. American Journal of Physical Medicine \& Rehabilitation, 74(5):S150-159, 1995. 3

[15] Clément Grondard, Olivier Biondi, Anne-Sophie Armand, Sylvie Lécolle, Bruno Della Gaspera, Claude Pariset, Hung Li, Claude-Louis Gallien, Pierre-Paul Vidal, Christophe Chanoine, and Frédéric Charbonnier. Regular exercise prolongs survival in a type 2 spinal muscular atrophy model mouse. The Journal of Neuroscience: The Official Journal of the Society for Neuroscience, 25(33):7615-7622, 2005. 3

[16] I. Vieitez, P. Gallano, L. González-Quereda, S. Borrego, I. Marcos, J. M. Millán, T. Jairo, C. Prior, J. Molano, M. J. Trujillo-Tiebas, J. Gallego-Merlo, M. García-Barcina, M. Fenollar, and C. Navarro. Mutational spectrum of 
Duchenne muscular dystrophy in Spain: study of 284 cases. Neurología (English Edition), 32(6):377-385, July 2017. 4

[17] W. Ian McDonald, Alistair Compston, Gilles Edan, Donald Goodkin, HansPeter Hartung, Fred D. Lublin, Henry F. McFarland, Donald W. Paty, Chris H. Polman, Stephen C. Reingold, and others. Recommended diagnostic criteria for multiple sclerosis: guidelines from the International Panel on the diagnosis of multiple sclerosis. Annals of neurology, 50(1):121127, 2001. 4

[18] E. Leray, T. Moreau, A. Fromont, and G. Edan. Epidemiology of multiple sclerosis. Revue Neurologique, 172(1):3-13, January 2016. 4

[19] Ralph L. Sacco, Scott E. Kasner, Joseph P. Broderick, Louis R. Caplan, Antonio Culebras, Mitchell SV Elkind, Mary G. George, Allen D. Hamdan, Randall T. Higashida, Brian L. Hoh, and others. An updated definition of stroke for the 21st century. Stroke, 44(7):2064-2089, 2013. 4

[20] Bruce Ovbiagele and Mai N. Nguyen-Huynh. Stroke Epidemiology: Advancing Our Understanding of Disease Mechanism and Therapy. Neurotherapeutics, 8(3):319-329, July 2011. 4

[21] T. Truelsen, B. Piechowski-Jóźwiak, R. Bonita, C. Mathers, J. Bogousslavsky, and G. Boysen. Stroke incidence and prevalence in Europe: a review of available data. European journal of neurology, 13(6):581-598, 2006. 4

[22] Peter Rosenbaum, Nigel Paneth, Alan Leviton, Murray Goldstein, Martin Bax, Diane Damiano, Bernard Dan, Bo Jacobsson, and others. A report: the definition and classification of cerebral palsy April 2006. Dev Med Child Neurol Suppl, 109(109):8-14, 2007. 5

[23] Ingo Borggraefe, Jan Simon Schaefer, Mirjam Klaiber, Edward Dabrowski, Corinne Ammann-Reiffer, Beat Knecht, Steffen Berweck, Florian Heinen, and Andreas Meyer-Heim. Robotic-assisted treadmill therapy improves walking and standing performance in children and adolescents with cerebral 
palsy. European journal of paediatric neurology: EJPN: official journal of the European Paediatric Neurology Society, 14(6), 2010. 5

[24] Nicola Smania, Paola Bonetti, Marialuisa Gandolfi, Alessandro Cosentino, Andreas Waldner, Stefan Hesse, Cordula Werner, Giulia Bisoffi, Christian Geroin, and Daniele Munari. Improved gait after repetitive locomotor training in children with cerebral palsy. American Journal of Physical Medicine \& Rehabilitation, 90(2):137-149, 2011. 5

[25] Else Odding, Marij E. Roebroeck, and Hendrik J. Stam. The epidemiology of cerebral palsy: incidence, impairments and risk factors. Disability and Rehabilitation, 28(4):183-191, February 2006. 5

[26] Frederick M. Maynard, Michael B. Bracken, GJFD Creasey, John F. Ditunno, William H. Donovan, Thomas B. Ducker, Susan L. Garber, Ralph J. Marino, Samuel L. Stover, Charles H. Tator, and others. International standards for neurological and functional classification of spinal cord injury. Spinal cord, 35(5):266-274, 1997. 5

[27] Anoushka Singh, Lindsay Tetreault, Suhkvinder Kalsi-Ryan, Aria Nouri, and Michael G Fehlings. Global prevalence and incidence of traumatic spinal cord injury. Clinical Epidemiology, 6(1):309-331, 2014. 5

[28] Daniel P. Ferris, Gregory S. Sawicki, and Antoinette R. Domingo. Powered lower limb orthoses for gait rehabilitation. Topics in spinal cord injury rehabilitation, 11(2):34, 2005. 6, 10, 14, 114

[29] Rong-Ju Cherng, Chuan-Fei Liu, Tin-Wai Lau, and Rong-Bin Hong. Effect of treadmill training with body weight support on gait and gross motor function in children with spastic cerebral palsy. American journal of physical medicine \& rehabilitation, 86(7):548-555, 2007. 7

[30] Anton Wernig and S. Müller. Laufband locomotion with body weight support improved walking in persons with severe spinal cord injuries. Spinal Cord, 30(4):229-238, 1992. 7 
[31] Ray D. de Leon, Roland R. Roy, and V. Reggie Edgerton. Is the recovery of stepping following spinal cord injury mediated by modifying existing neural pathways or by generating new pathways? a perspective. Physical therapy, 81(12):1904-1911, 2001. 7

[32] Hami Kazerooni, J.-L. Racine, Lihua Huang, and Ryan Steger. On the control of the berkeley lower extremity exoskeleton (BLEEX). In Robotics and Automation, 2005. ICRA 2005. Proceedings of the 2005 IEEE International Conference on, page 4353-4360. IEEE, 2005. 8

[33] Adam B. Zoss, Hami Kazerooni, and Andrew Chu. Biomechanical design of the berkeley lower extremity exoskeleton (BLEEX). Mechatronics, IEEE/ASME Transactions on, 11(2):128-138, 2006. 8

[34] John M. Hollerbach, Yangming Xu, R. Christensen, Stephen C. Jacobsen, and others. Design specifications for the second generation Sarcos Treadport locomotion interface. In Haptics Symposium, pages 1293-1298, 2000. 8

[35] K. H. Low, X. Liu, and H. Yu. Design and implementation of NTU wearable exoskeleton as an enhancement and assistive device. Applied Bionics and Biomechanics, 3(3):209-225, 2006. 8

[36] Hiroaki Kawamoto and Yoshiyuki Sankai. Power assist method based on phase sequence and muscle force condition for HAL. Advanced Robotics, 19(7):717-734, 2005. 8

[37] Sunil K. Agrawal, Sai K. Banala, Abbas Fattah, Vivek Sangwan, Vijaya Krishnamoorthy, John P. Scholz, and Hsu Wei-Li. Assessment of motion of a swing leg and gait rehabilitation with a gravity balancing exoskeleton. Neural Systems and Rehabilitation Engineering, IEEE Transactions on, 15(3):410-420, 2007. 10, 11

[38] Joseph Hidler, Diane Nichols, Marlena Pelliccio, Kathy Brady, Donielle D. Campbell, Jennifer H. Kahn, and T. George Hornby. Multicenter randomized clinical trial evaluating the effectiveness of the lokomat in subacute stroke. Neurorehabilitation and Neural Repair, 23(1):5-13, 2009. 10 
[39] Joseph Hidler, Wessel Wisman, and Nathan Neckel. Kinematic trajectories while walking within the lokomat robotic gait-orthosis. Clinical Biomechanics, 23(10):1251-1259, 2008. 11

[40] Jan F. Veneman, Rik Kruidhof, Edsko EG Hekman, Ralf Ekkelenkamp, Edwin HF Van Asseldonk, and Herman Van Der Kooij. Design and evaluation of the LOPES exoskeleton robot for interactive gait rehabilitation. Neural Systems and Rehabilitation Engineering, IEEE Transactions on, 15(3):379-386, 2007. 11,62

[41] Sai K. Banala, Seok Hun Kim, Sunil K. Agrawal, and John P. Scholz. Robot assisted gait training with active leg exoskeleton (ALEX). Neural Systems and Rehabilitation Engineering, IEEE Transactions on, 17(1):2-8, 2009. 11

[42] Mohamed Bouri, Yves Stauffer, Carl Schmitt, Yves Allemand, Stany Gnemmi, Reymond Clavel, Patrick Metrailler, and Roland Brodard. The walktrainer: a robotic system for walking rehabilitation. In IEEE International Conference on Robotics and Biomimetics, page 1616-1621, 2006. 11

[43] Aaron M. Dollar and Hugh Herr. Active orthoses for the lower-limbs: challenges and state of the art. In Rehabilitation Robotics, 2007. ICORR 2007. IEEE 10th International Conference on, pages 968-977. IEEE, 2007. 13

[44] Rewalk. http://rewalk.com. Accessed: 2014-07-08. 13,99

[45] Rex bionics. http: //www.rexbionics. com. Accessed: 2014-07-08. 13,14

[46] Ekso bionics. http://WWW.eksobionics.com/ekso, Accessed: 2014-07-08. 13, 14, 99

[47] Peter D. Neuhaus, Jerryll H. Noorden, Travis J. Craig, Tecalote Torres, Justin Kirschbaum, and Jerry E. Pratt. Design and evaluation of mina: A robotic orthosis for paraplegics. In Rehabilitation Robotics (ICORR), 2011 IEEE International Conference on, page 1-8. IEEE, 2011. 15, 62 
[48] Conor James Walsh, Ken Endo, and Hugh Herr. A quasi-passive leg exoskeleton for load-carrying augmentation. International Journal of Humanoid Robotics, pages 487-506, 2007. 16

[49] Conor James Walsh, Daniel Paluska, Kenneth Pasch, William Grand, Andrew Valiente, and Hugh Herr. Development of a lightweight, underactuated exoskeleton for load-carrying augmentation. In Robotics and Automation, 2006. ICRA 2006. Proceedings 2006 IEEE International Conference on, pages 3485-3491. IEEE, 2006. 16

[50] Andrew (Andrew J. ) Valiente. Design of a quasi-passive parallel leg exoskeleton to augment load carrying for walking. Thesis, Massachusetts Institute of Technology, 2005. 16,92

[51] D. Sanz-Merodio, M. Cestari, J. C. Arevalo, X. Carrillo, and E. Garcia. Development of a Lower-Limb Active Orthosis and a Walker for Gait Assistance. In ROBOT2013: First Iberian Robotics Conference, Advances in Intelligent Systems and Computing, pages 219-233. Springer International Publishing, 2014. 19

[52] R. McNeil Alexander. Energy-saving mechanisms in walking and running. Journal of Experimental Biology, 160(1):55-69, 1991. 23

[53] Reinhard Blickhan. The spring-mass model for running and hopping. Journal of biomechanics, 22(11):1217-1227, 1989. 26

[54] Thomas A. McMahon and George C. Cheng. The mechanics of running: how does stiffness couple with speed? Journal of biomechanics, 23(1):6578, 1990. 26

[55] A. D. Kuo. Choosing your steps carefully. Robotics and Automation Magazine, IEEE, 14(2):18-29, 2007. 26, 93

[56] J. M Donelan, R. Kram, and A. D Kuo. Simultaneous positive and negative external mechanical work in human walking. Journal of Biomechanics, 35(1):117-124, 2002. 26, 101 
[57] Hartmut Geyer, Andre Seyfarth, and Reinhard Blickhan. Compliant leg behaviour explains basic dynamics of walking and running. Proceedings of the Royal Society B: Biological Sciences, 273(1603):2861-2867, 2006. 27

[58] Arthur D. Kuo. The six determinants of gait and the inverted pendulum analogy: A dynamic walking perspective. Human movement science, 26(4):617-656, 2007. 27

[59] Peter G. Adamczyk, Steven H. Collins, and Arthur D. Kuo. The advantages of a rolling foot in human walking. Journal of Experimental Biology, 209(20):3953-3963, 2006. 27

[60] Jacquelin Perry. Gait Analysis: Normal and Pathological Function. January 1992. 30

[61] Manoj Srinivasan and Andy Ruina. Computer optimization of a minimal biped model discovers walking and running. Nature, 439(7072):72-75, 2005. 32

[62] Steven H. Collins, Peter G. Adamczyk, and Arthur D. Kuo. Dynamic arm swinging in human walking. Proceedings of the Royal Society of London B: Biological Sciences, 276(1673):3679-3688, 2009. 32

[63] Jessica Rose, James Gibson Gamble, and Janet M. Adams. Human walking. Lippincott Williams \& Wilkins Philadelphia, 2006. 33

[64] R.W. Baker. Measuring Walking: A Handbook of Clinical Gait Analysis. Wiley, 2013. 38

[65] Frank C. Anderson and Marcus G. Pandy. Individual muscle contributions to support in normal walking. Gait \& Posture, 17(2):159-169, April 2003. 44

[66] David A. Winter. Foot trajectory in human gait: a precise and multifactorial motor control task. Physical therapy, 72(1):45-53, 1992. 46 
[67] A. V. Hill. The heat of shortening and the dynamic constants of muscle. Proceedings of the Royal Society of London. Series B - Biological Sciences, 126(843):136-195, October 1938. 50

[68] A. F. Huxley and R. M. Simmons. Proposed Mechanism of Force Generation in Striated Muscle. Nature, 233(5321):533-538, October 1971. 50

[69] Miomir Vukobratović and Branislav Borovac. Zero-moment point—-thirty five years of its life. International journal of humanoid robotics, 1(01):157173, 2004. 56

[70] Masato Hirose and Kenichi Ogawa. Honda humanoid robots development. Philosophical Transactions of the Royal Society of London A: Mathematical, Physical and Engineering Sciences, 365(1850):11-19, 2007. 56

[71] Steve Collins, Andy Ruina, Russ Tedrake, and Martijn Wisse. Efficient bipedal robots based on passive dynamic walkers. Science, 307(5712):10821085, 2005. 56, 70, 92

[72] J. Maxwell Donelan, Rodger Kram, and Arthur D. Kuo. Mechanical work for step-to-step transitions is a major determinant of the metabolic cost of human walking. Journal of Experimental Biology, 205(23):3717-3727, 2002. 56

[73] S. Mochon and T. A McMahon. Ballistic walking. Journal of Biomechanics, 13(1):49-57, 1980. 56, 85

[74] Tad McGeer. Passive walking with knees. In Robotics and Automation, 1990. Proceedings., 1990 IEEE International Conference on, pages 16401645. IEEE, 1990. 56

[75] Martijn Wisse. Three additions to passive dynamic walking: actuation, an upper body, and 3d stability. International Journal of Humanoid Robotics, 2(04):459-478, 2005. 56 
[76] Daan Hobbelen, Tomas de Boer, and Martijn Wisse. System overview of bipedal robots flame and tulip: Tailor-made for limit cycle walking. In 2008 IEEE/RSJ International Conference on Intelligent Robots and Systems, pages 2486-2491. IEEE, 2008. 57

[77] Martijn Wisse, Guillaume Keliksdal, Jan Van Frankenhyyzen, and Brian Moyer. Passive-based walking robot. IEEE Robotics \& Automation Magazine, 14(2):52-62, 2007. 57

[78] VIACTORS - Variable Impedance Actuation Systems Embodying Advanced Interaction Behaviours. http://www.viactors.org. Accessed: 2017-10-18. 61, 125

[79] R. Ham, T. Sugar, B. Vanderborght, K. Hollander, and D. Lefeber. Compliant actuator designs. Robotics \& Automation Magazine, IEEE, 16(3):81-94, 2009. 61, 96, 111

[80] B. Vanderborght, A. Albu-Schaeffer, A. Bicchi, E. Burdet, D.G. Caldwell, R. Carloni, M. Catalano, O. Eiberger, W. Friedl, G. Ganesh, M. Garabini, M. Grebenstein, G. Grioli, S. Haddadin, H. Hoppner, A. Jafari, M. Laffranchi, D. Lefeber, F. Petit, S. Stramigioli, N. Tsagarakis, M. Van Damme, R. Van Ham, L.C. Visser, and S. Wolf. Variable impedance actuators: A review. 61(12):1601-1614, 2013. 61

[81] Jerry E. Pratt and Benjamin T. Krupp. Series Elastic Actuators for legged robots. pages 135-145. International Society for Optics and Photonics, September 2004. 61

[82] Marco Hutter, C. David Remy, Mark A. Hoepflinger, and Roland Siegwart. High compliant series elastic actuation for the robotic leg ScarlETH. Proc. of the International Conference on Climbing and Walking Robots (CLAWAR), 2011. 62

[83] Shane A. Migliore, Edgar A. Brown, and Stephen P. DeWeerth. Biologically inspired joint stiffness control. In Robotics and Automation, 2005. ICRA 2005. Proceedings of the 2005 IEEE International Conference on, pages 4508-4513. IEEE, 2005. 62, 117 
[84] B. Vanderborght, B. Verrelst, R. Van Ham, M. Van Damme, P. Beyl, and D. Lefeber. Development of a compliance controller to reduce energy consumption for bipedal robots. Autonomous Robots, 24(4):419-434, 2008. 63 . 96

[85] Jonathan W. Hurst, Joel E. Chestnutt, and Alfred A. Rizzi. An actuator with physically variable stiffness for highly dynamic legged locomotion. pages 4662-4667. Robotics and Automation, ICRA'04. IEEE, 2004. 63

[86] Oliver Eiberger, Sami Haddadin, Michael Weis, Alin Albu-Schäffer, and Gerd Hirzinger. On joint design with intrinsic variable compliance: Derivation of the DLR QA-joint. pages 1687-1694. Robotics and Automation (ICRA), 2010 IEEE International Conference on, 2010. 63

[87] Riccardo Schiavi, Giorgio Grioli, Soumen Sen, and Antonio Bicchi. VSA-II: A novel prototype of variable stiffness actuator for safe and performing robots interacting with humans. pages 2171-2176. Robotics and Automation, ICRA 2008. IEEE International Conference on, 2008. 65, 68, 117

[88] A. Jafari, N. G Tsagarakis, B. Vanderborght, and D. G Caldwell. A novel actuator with adjustable stiffness (AwAS). page 4201-4206. Intelligent Robots and Systems (IROS), 2010 IEEE/RSJ International Conference on, 2010. 67. 96, 114,117

[89] Nikos G. Tsagarakis, Irene Sardellitti, and Darwin G. Caldwell. A new variable stiffness actuator (CompAct-VSA): Design and modelling. pages 378383. Intelligent Robots and Systems (IROS), 2011 IEEE/RSJ International Conference on, 2011. 67

[90] L. C. Visser, R. Carloni, and S. Stramigioli. Variable stiffness actuators: A port-based analysis and a comparison of energy efficiency. In Robotics and Automation (ICRA), 2010 IEEE International Conference on, page 3279-3284, 2010. 67, 126

[91] Amir Jafari, Nikos G. Tsagarakis, and Darwin G. Caldwell. A novel intrinsically energy efficient actuator with adjustable stiffness (AwAS). 18(1):355365, 2013. 68 
[92] Jerry Pratt, Chee-Meng Chew, Ann Torres, Peter Dilworth, and Gill Pratt. Virtual model control: An intuitive approach for bipedal locomotion. The International Journal of Robotics Research, 20(2):129-143, 2001. 71

[93] Eric R. Westervelt, Jessy W. Grizzle, and Daniel E. Koditschek. Hybrid zero dynamics of planar biped walkers. IEEE transactions on automatic control, pages $42-56,2003.71$

[94] Mark W. Spong and Francesco Bullo. Controlled symmetries and passive walking. IFAC Proceedings Volumes, pages 557-562, 2002. 71

[95] Aaron D. Ames, Robert D. Gregg, Eric DB Wendel, and Shankar Sastry. On the geometric reduction of controlled three-dimensional bipedal robotic walkers. Lecture Notes in Control and Information Sciences, 366(1):183, 2007. 71

[96] E. R. Westervelt, B. Morris, and K. D. Farrell. Analysis results and tools for the control of planar bipedal gaits using hybrid zero dynamics. Autonomous Robots, 23(2):131-145, 2007. 71

[97] R. Dumas, L. Cheze, and J.P. Verriest. Adjustments to McConville et al. and young et al. body segment inertial parameters. Journal of biomechanics, 40(3):543-553, 2007. 75

[98] Yobotics Inc., Boston, MA. Yobotics! Simulation Construction Set: Users Guide, 2002. Available: http://www - yobotics.com/. 75

[99] Jeffrey C. Lagarias, James A. Reeds, Margaret H. Wright, and Paul E. Wright. Convergence properties of the nelder-mead simplex Method in low dimensions. SIAM Journal on Optimization, 9(1):112-147, 1998. 79

[100] J. A. Nelder and R. Mead. A simplex method for function minimization. The Computer Journal, pages 308-313, 1965. 79

[101] Willi-Hans Steeb. The nonlinear workbook: chaos, fractals, cellular automata, genetic algorithms, gene expression programming, support vector machine, wavelets, hidden arkov models, fuzzy logic with $C++$, Java and $C$ programs. World Scientific Publishing Co Inc, 2014. 80 
[102] G. A Cavagna, N. C Heglund, and C. R Taylor. Mechanical work in terrestrial locomotion: two basic mechanisms for minimizing energy expenditure. American Journal of Physiology-Regulatory, Integrative and Comparative Physiology, 233(5):243, 1977. 85

[103] Emery N Brown, Hugh M Herr, and Pavitra Krishnaswamy. Human leg model predicts ankle Muscle-Tendon morphology, state, roles and energetics in walking. Computational Biology, 7(3):16, 2010. 87

[104] G. Munoz, M. Cestari, J. Arevalo, D. Sanz-Merodio, and E. Garcia. Benefits and drawbacks of mrf knee damping in legged robots. Adaptive Mobile Robotics, 1(1):384-389, 2012. 92

[105] B. Vanderborght, N. G Tsagarakis, C. Semini, R. Van Ham, and D. G Caldwell. Maccepa 2.0: Adjustable compliant actuator with stiffening characteristic for energy efficient hopping. In Robotics and Automation, 2009. ICRA'09. IEEE International Conference on, pages 544-549, 2009. 96

[106] J. W Hurst, J. E Chestnutt, and A. A Rizzi. The actuator with mechanically adjustable series compliance. Robotics, IEEE Transactions on, 26(4):1-10, 2010. 96, 114,117

[107] R. Van Ham, B. Vanderborght, M. Van Damme, B. Verrelst, and D. Lefeber. MACCEPA, the mechanically adjustable compliance and controllable equilibrium position actuator: Design and implementation in a biped robot. Robotics and Autonomous Systems, 55(10):761-768, 2007. 96, 114, 117

[108] S. Wolf and G. Hirzinger. A new variable stiffness design: Matching requirements of the next robot generation. In Robotics and Automation, 2008. ICRA 2008. IEEE International Conference on, pages 1741-1746, 2008. 96. 114,117

[109] R. Farris, H. Quintero, and M. Goldfarb. Preliminary evaluation of a powered lower limb orthosis to aid walking in paraplegic individuals. Neural Systems and Rehabilitation Engineering, IEEE Transactions on, 19(6):652-659, 2011. 99 
[110] Jesse R. Modica and Rodger Kram. Metabolic energy and muscular activity required for leg swing in running. Journal of Applied Physiology, 98(6):2126-2131, June 2005. 100

[111] Raymond C. Browning, Jesse R. Modica, Rodger Kram, Ambarish Goswami, and others. The effects of adding mass to the legs on the energetics and biomechanics of walking. Medicine and science in sports and exercise, 39(3):515, 2007. 100

[112] A. J Van Den Bogert. Exotendons for assistance of human locomotion. Biomedical engineering online, 2(17), 2003. 100

[113] Juan Carlos Arevalo, Daniel Sanz-Merodio, Manuel Cestari, and Elena Garcia. Parameterized inverted and double pendulum model for controlling lower-limb active orthosis. pages 1899-1905. 2012 IEEE International Conference on Robotics and Biomimetics (ROBIO), 2012. 107

[114] A. Albu-Schaffer and G. Hirzinger. Cartesian impedance control techniques for torque controlled light-weight robots. In Proceedings 2002 IEEE International Conference on Robotics and Automation, volume 1, pages 657 663 vol.1, 2002. 111

[115] D. Sanz-Merodio, M. Cestari, J. C. Arevalo, and E. Garcia. A lower-limb exoskeleton for gait assistance in quadriplegia. In 2012 IEEE International Conference on Robotics and Biomimetics (ROBIO), pages 122-127, Guangzhou,China, December 2012. 112

[116] A. M. Dollar and H. Herr. Lower extremity exoskeletons and active orthoses: Challenges and state-of-the-art. Robotics, IEEE Transactions on, page $144-158,2008$. 112,179

[117] Ruta Desai and Hartmut Geyer. Robust swing leg placement under large disturbances. In 2012 IEEE International Conference on Robotics and Biomimetics (ROBIO), , pages 265-270, 2012. 120 
[118] Creo Parametric 3d Modeling Software | PTC. https://www.ptc. com/en/products/cad/creo/parametric. Accessed: 2018-0626. 130

[119] Daniel Sanz-Merodio, Manuel Cestari, Juan Carlos, and Elena Garcia. Control motion approach of a lower limb orthosis to reduce energy consumption. International Journal of Advanced Robotic Systems, 9(6):232, 2012. 143

[120] Y. Yavin. Control of a three-link manipulator with a constraint on the velocity of its end-effector. Computers \& Mathematics with Applications, 40(10):1263-1273, 2000. 186

[121] Joseph F. Cusick, Narayan Yoganandan, Frank Pintart, Joel Myklebust, and Hassan Hussain. Biomechanics of cervical spine facetectomy and fixation techniques. 13(7):808-812, 1988. 187

[122] Nancy P. Hamilton. Kinesiology: scientific basis of human motion. Brown \& Benchmark, 2011. 187

[123] Elena Garcia and Pablo Gonzalez de Santos. On the improvement of walking performance in natural environments by a compliant adaptive gait. 22(6):1240-1253, 2006. 191

[124] W. Y. Jiang, A. M. Liu, and D. Howard. Optimization of legged robot locomotion by control of foot-force distribution. 26(4):311-323, 2004. 193 


\section{Declaration}

I herewith declare that I have produced this work without the prohibited assistance of third parties and without making use of aids other than those specified; notions taken over directly or indirectly from other sources have been identified as such. This work has not previously been presented in identical or similar form to any examination board.

The dissertation work was conducted from 2012 to 2018 under the supervision of Elena García Armada at the Universidad Politécnica de Madrid.

Madrid, 
This dissertation was finished writing in Madrid on Thursday 28th June, 2018 
This page is intentionally left blank 